Ricardo Almeida, Dina Tavares, Delfim F. M. Torres

\title{
The Variable-Order Fractional Calculus of Variations
}

June 17, 2018

This is a preprint. The final authenticated version is available online as a SpringerBrief in Applied Sciences and Technology at: https://doi.org/10.1007/978-3-319-94006-9

Please cite this book as follows:

R. Almeida, D. Tavares and D. F. M. Torres,

The variable-order fractional calculus of variations,

SpringerBriefs in Applied Sciences and Technology, Springer, Cham, 2019. 



\section{Preface}

This book intends to deepen the study of the fractional calculus, giving special emphasis to variable-order operators.

Fractional calculus is a recent field of mathematical analysis and it is a generalization of integer differential calculus, involving derivatives and integrals of real or complex order (Kilbas. Srivastava and Trujillo, 2006; Podlubnv, 1999). The first note about this ideia of differentiation, for non-integer numbers, dates back to 1695, with a famous correspondence between Leibniz and L'Hôpital. In a letter, L'Hôpital asked Leibniz about the possibility of the order $n$ in the notation $d^{n} y / d x^{n}$, for the $n$th derivative of the function $y$, to be a non-integer, $n=1 / 2$. Since then, several mathematicians investigated this approach, like Lacroix, Fourier, Liouville, Riemann, Letnikov, Grünwald, Caputo, and contributed to the grown development of this field. Currently, this is one of the most intensively developing areas of mathematical analysis as a result of its numerous applications. The first book devoted to the fractional calculus was published by Oldham and Spanier in 1974, where the authors systematized the main ideas, methods and applications about this field (Mainardi, 2010).

In the recent years, fractional calculus has attracted the attention of many mathematicians, but also some researchers in other areas like physics, chemistry and engineering. As it is well known, several physical phenomena are often better described by fractional derivatives (Herrmann, 2013; Odzijewicz, Malinowska and Torres, 2012a; Sheng, 2012). This is mainly due to the fact that fractional operators take into consideration the evolution of the system, by taking the global correlation, and not only local characteristics. Moreover, integer-order calculus sometimes contradict the experimental results and therefore derivatives of fractional order may be more suitable (Hilfer, 2000).

In 1993, Samko and Ross devoted themselves to investigate operators when the order $\alpha$ is not a constant during the process, but variable on time: $\alpha(t)$ (Samko and Ross, 1993). An interesting recent generalization of the theory of fractional calculus is developed to allow the fractional order of the derivative to be non-constant, depending on time (Chen, Liu and Burrage, 
2014; Odzilewicz. Malinowska and Torres, 2012b, 2013a). With this approach of variable-order fractional calculus, the non-local properties are more evident and numerous applications have been found in physics, mechanics, control and signal processing (Coimbra. Soon and Kobavashi, 2005; Ingman and Suzdalnitsky, 2004; Odzijewicz. Malinowska and Torres, 2013b; Ostalczyk et al., 2015; Ramirez and Coimbra, 2011; Rapaić and Pisano, 2014; Valério and Costa, 2013).

Although there are many definitions of fractional derivative, the most commonly used are the Riemann-Liouville, the Caputo, and the GrünwaldLetnikov derivatives. For more about the development of fractional calculus, we suggest (Samko, Kilbas and Marichev, 1993), (Samko and Ross, 1993), (Podlubny, 1999), (Kilbas, Srivastava and Trujillo, 2006) or (Mainardi, 2010).

One difficult issue that usually arises when dealing with such fractional operators, is the extreme difficulty in solving analytically such problems (Atangana and Cloot, 2013; Zhuang et al., 2009). Thus, in most cases, we do not know the exact solution for the problem and one needs to seek a numerical approximation. Several numerical methods can be found in the literature, typically applying some discretization over time or replacing the fractional operators by a proper decomposition (Atangana and Cloot, 2013; Zhuang et al., 2009).

Recently, new approximation formulas were given for fractional constant order operators, with the advantage that higher-order derivatives are not required to obtain a good accuracy of the method (Atanacković et al., 2013; Pooseh, Almeida and Torres, 2012, 2013). These decompositions only depend on integer-order derivatives, and by replacing the fractional operators that appear in the problem by them, one leaves the fractional context ending up in the presence of a standard problem, where numerous tools are available to solve them (Almeida, Pooseh and Torres, 2015).

The first goal of this book is to extend such decompositions to Caputo fractional problems of variable-order. For three types of Caputo derivatives with variable-order, we obtain approximation formulas for the fractional operators and respective upper bounds for the errors.

Then, we focus our attention on a special operator introduced by Malinowska and Torres: the combined Caputo fractional derivative, which is an extension of the left and the right fractional Caputo derivatives (Malinowska and Torres, 2010). Considering $\alpha, \beta \in(0,1)$ and $\gamma \in[0,1]$, the combined Caputo fractional derivative operator ${ }^{C} D_{\gamma}^{\alpha, \beta}$ is a convex combination of the left and the right Caputo fractional derivatives, defined by

$$
{ }^{C} D_{\gamma}^{\alpha, \beta}=\gamma_{a}^{C} D_{t}^{\alpha}+(1-\gamma){ }_{t}^{C} D_{b}^{\beta}
$$

We consider this fractional operator with variable fractional order, i.e., the combined Caputo fractional derivative of variable-order:

$$
{ }^{C} D_{\gamma}^{\alpha(\cdot, \cdot), \beta(\cdot, \cdot)} x(t)=\gamma_{1}{ }_{a}^{C} D_{t}^{\alpha(\cdot, \cdot)} x(t)+\gamma_{2}{ }_{t}^{C} D_{b}^{\beta(\cdot, \cdot)} x(t),
$$


where $\gamma=\left(\gamma_{1}, \gamma_{2}\right) \in[0,1]^{2}$, with $\gamma_{1}$ and $\gamma_{2}$ not both zero. With this fractional operator, we study different types of fractional calculus of variations problems, where the Lagrangian depends on the referred derivative.

The calculus of variations is a mathematical subject that appeared formally in the XVII century, with the solution to the bachistochrone problem, that deals with the extremization (minimization or maximization) of functionals (van Brunt, 2004). Usually, functionals are given by an integral that involves one or more functions or/and its derivatives. This branch of mathematics has proved to be relevant because of the numerous applications existing in real situations.

The fractional variational calculus is a recent mathematical field that consists in minimizing or maximizing functionals that depend on fractional operators (integrals or/and derivatives). This subject was introduced by Riewe in 1996, where the author generalizes the classical calculus of variations, by using fractional derivatives, and allows to obtain conservations laws with nonconservative forces such as friction (Riewe, 1996, 1997). Later appeared several works on various aspects of the fractional calculus of variations and involving different fractional operators, like the Riemann-Liouville, the Caputo, the Grun̈wald-Letnikov, the Weyl, the Marchaud or the Hadamard fractional derivatives (Agrawal, 2002; Almeida, 2016; Askari and Ansari, 2016; Atanacković, Koniik and Pilipović, 2008; Baleanu, 2008; Fraser, 1992; Georgieva and Guenther, 2002; Jarad, Abdeljawad and Baleanu, 2010). For the state of the art of the fractional calculus of variations, we refer the readers to the books (Almeida, Pooseh and Torres, 2015; Malinowska. Odzijewicz and Torres, 2015; Malinowska and Torres, 2012).

Specifically, here we study some problems of the calculus of variations with integrands depending on the independent variable $t$, an arbitrary function $x$ and a fractional derivative ${ }^{C} D_{\gamma}^{\alpha(\cdot, \cdot), \beta(\cdot, \cdot)} x$. The endpoint of the cost integral, as well the terminal state, are considered to be free. The fractional problem of the calculus of variations consists in finding the maximizers or minimizers to the functional

$$
\mathcal{J}(x, T)=\int_{a}^{T} L\left(t, x(t),{ }^{C} D_{\gamma}^{\alpha(\cdot, \cdot), \beta(\cdot, \cdot)} x(t)\right) d t+\phi(T, x(T)),
$$

where ${ }^{C} D_{\gamma}^{\alpha(\cdot, \cdot), \beta(\cdot, \cdot)} x(t)$ stands for the combined Caputo fractional derivative of variable fractional order, subject to the boundary condition $x(a)=x_{a}$. For all variational problems presented here, we establish necessary optimality conditions and transversality optimality conditions.

The book is organized in two parts, as follows. In the first part, we review the basic concepts of fractional calculus (Chapter 1) and of the fractional calculus of variations (Chapter 2). In Chapter 1) we start with a brief overview about fractional calculus and an introduction to the theory of some special functions in fractional calculus. Then, we recall several fractional operators (integrals and derivatives) definitions and some properties of the considered fractional derivatives and integrals are introduced. In the end of this chapter, 
we review integration by parts formulas for different operators. Chapter 2 presents a short introduction to the classical calculus of variations and review different variational problems, like the isoperimetric problems or problems with variable endpoints. In the end of this chapter, we introduce the theory of the fractional calculus of variations and some fractional variational problems with variable-order.

In the second part, we systematize some new recent results on variableorder fractional calculus of (Tavares. Almeida and Torres, 2015, 2016, 2017, 2018a,b). In Chapter 3, considering three types of fractional Caputo derivatives of variable-order, we present new approximation formulas for those fractional derivatives and prove upper bound formulas for the errors. In Chapter 4 . we introduce the combined Caputo fractional derivative of variable-order and corresponding higher-order operators. Some properties are also given. Then, we prove fractional Euler-Lagrange equations for several types of fractional problems of the calculus of variations, with or without constraints.

\section{References}

Agrawal OP (2002) Formulation of Euler-Lagrange equations for fractional variational problems. J. Math. Anal. Appl. 272:368-379

Almeida R (2016) Fractional variational problems depending on indefinite integrals and with delay. Bull. Malays. Math. Sci. Soc. 39(4):1515-1528 arXiv: 1512.06752

Almeida R, Pooseh S, Torres DFM (2015) Computational Methods in the Fractional Calculus of Variations. Imperial College Press, London

Askari H, Ansari A (2016) Fractional calculus of variations with a generalized fractional derivative. Fract. Differ. Calc. 6:57-72

Atanacković TM, Janev M, Pilipović S, Zorica D (2013) An expansion formula for fractional derivatives of variable order. Cent. Eur. J. Phys. 11(10):13501360

Atanacković TM, Konjik S, Pilipović S (2008) Variational problems with fractional derivatives: Euler-Lagrange equations. J. Phys. A 41(9):095201, $12 \mathrm{pp}$

Atangana A, Cloot AH (2013) Stability and convergence of the space fractional variable-order Schrödinger equation. Adv. Difference Equ. 80(1):10 pp

Baleanu D (2008) New applications of fractional variational principles. Rep. Math. Phys. 61(2):199-206

Chen S, Liu F, Burrage K (2014) Numerical simulation of a new two-dimensional variable-order fractional percolation equation in nonhomogeneous porous media. Comput. Math. Appl. 67(9):1673-1681

Coimbra CFM, Soon CM, Kobayashi MH (2005) The variable viscoelasticity operator. Annalen der Physik 14(6):378-389

Fraser C (1992) Isoperimetric problems in variatonal calculus of Euler and Lagrange. Historia Mathematica 19:4-23 
Georgieva B, Guenther RB (2002) First Noether-type theorem for the generalized variational principle of Herglotz. Topol. Methods Nonlinear Anal. 20(2):261-273

Herrmann R (2013) Folded potentials in cluster physics-a comparison of Yukawa and Coulomb potentials with Riesz fractional integrals. J. Phys. A 46(40):405203, $12 \mathrm{pp}$

Hilfer R (2000) Applications of fractional calculus in physics. World Sci. Publishing, River Edge, NJ

Ingman D, Suzdalnitsky J (2004) Control of damping oscillations by fractional differential operator with time-dependent order. Comput. Meth. Appl. Mech. Eng. 193(52):5585-5595

Jarad F, Abdeljawad T, Baleanu D (2010) Fractional variational principles with delay within Caputo derivatives. Rep. Math. Phys. 65(1):17-28

Kilbas AA, Srivastava HM, Trujillo JJ (2006) Theory and Applications of Fractional Differential Equations. Elsevier, Amsterdam

Mainardi F (2010) Fractional Calculus and Waves in Linear Viscoelasticity. Imp. Coll. Press, London

Malinowska AB, Odzijewicz T, Torres DFM (2015) Advanced Methods in the Fractional Calculus of Variations. Springer Briefs in Applied Sciences and Technology, Springer, Cham

Malinowska AB, Torres DFM (2010) Fractional variational calculus in terms of a combined Caputo derivative. Proceedings of FDA'10, The 4th IFAC Workshop on Fractional Differentiation and its Applications, Badajoz, Spain, October 18-20, 2010 (Eds: I. Podlubny, B. M. Vinagre Jara, YQ. Chen, V. Feliu Batlle, I. Tejado Balsera):Article no. FDA10-084, 6 pp. arXiv:1007.0743

Malinowska AB, Torres DFM (2012) Introduction to the Fractional Calculus of Variations. Imp. Coll. Press, London

Odzijewicz T, Malinowska AB, Torres DFM (2012a) Fractional calculus of variations in terms of a generalized fractional integral with applications to physics. Abstr. Appl. Anal. 2012:Art. ID 871912, 24 pp arXiv:1203.1961

Odzijewicz T, Malinowska AB, Torres DFM (2012b) Variable order fractional variational calculus for double integrals. Proceedings of the 51st IEEE Conference on Decision and Control, December 10-13, 2012, Maui, Hawaii:Art. no. $6426489,6873-6878$. arXiv: 1209.1345

Odzijewicz T, Malinowska AB, Torres DFM (2013a) Fractional variational calculus of variable order. in Advances in harmonic analysis and operator theory:291-301, Oper. Theory Adv. Appl., Birkhäuser/Springer Basel AG, Basel arXiv: 1110.4141

Odzijewicz T, Malinowska AB, Torres DFM (2013b) Noether's theorem for fractional variational problems of variable order. Cent. Eur. J. Phys. 11(6):691-701 arXiv: 1303.4075

Ostalczyk PW, Duch P, Brzeziń ski DW, Sankowski D (2015) Order functions selection in the variable-, fractional-order PID controller. Advances in Modelling and Control of Non-integer-Order Systems, Lecture Notes in Electrical Engineering 320:159-170. 
Podlubny I (1999) Fractional Differential Equations. Academic Press, San Diego, CA

Pooseh S, Almeida R, Torres DFM (2012) Approximation of fractional integrals by means of derivatives. Comput. Math. Appl. 64(10):3090-3100 arXiv: 1201.5224

Pooseh S, Almeida R, Torres DFM (2013) Numerical approximations of fractional derivatives with applications. Asian J. Control 15(3):698-712 arXiv: 1208.2588

Ramirez LES, Coimbra CFM (2011) On the variable order dynamics of the nonlinear wake caused by a sedimenting particle. Phys. D 240(13):11111118

Rapaić MR, Pisano A (2014) Variable-order fractional operators for adaptive order and parameter estimation. IEEE Trans. Automat. Control 59(3):798 803

Riewe F (1996) Nonconservative Lagrangian and Hamiltonian mechanics. Phys. Rev. E (3) 53(2):1890-1899

Riewe F (1997) Mechanics with fractional derivatives. Phys. Rev. E (3) $55(3): 3581-3592$

Samko SG, Kilbas AA, Marichev OI (1993) Fractional Integrals and Derivatives. translated from the 1987 Russian original, Gordon and Breach, Yverdon

Samko SG, Ross B (1993) Integration and differentiation to a variable fractional order. Integral Transform. Spec. Funct. 1(4):277-300

Tavares D, Almeida R, Torres DFM (2015) Optimality conditions for fractional variational problems with dependence on a combined Caputo derivative of variable order. Optimization 64(6):1381-1391 arXiv:1501.02082

Tavares D, Almeida R, Torres DFM (2016) Caputo derivatives of fractional variable order: numerical approximations. Commun. Nonlinear Sci. Numer. Simul. 35:69-87 arXiv:1511.02017

Tavares D, Almeida R, Torres DFM (2017) Constrained fractional variational problems of variable order. IEEE/CAA Journal Automatica Sinica 4(1):8088 arXiv: 1606.07512

Tavares D, Almeida R, Torres DFM (2018a) Fractional Herglotz variational problem of variable order. Disc. Contin. Dyn. Syst. Ser. S 11(1):143-154. arXiv: 1703.09104

Tavares D, Almeida R, Torres DFM (2018b) Combined fractional variational problems of variable order and some computational aspects. J. Comput. Appl. Math. 339:374-388. arXiv:1704.06486

Valério D, Costa JS (2013) Variable order fractional controllers. Asian J. Control 15(3):648-657

van Brunt B (2004) The Calculus of Variations. Universitext, Springer, New York

Zheng B (2012) $\left(G^{\prime} / G\right)$-expansion method for solving fractional partial differential equations in the theory of mathematical physics. Commun. Theor. Phys. 58(5):623-630 
Zhuang P, Liu F, Anh V, Turner I (2009) Numerical methods for the variableorder fractional advection-diffusion equation with a nonlinear source term. SIAM J. Numer. Anal. 47(3):1760-1781. 



\section{Acknowledgements}

This work was supported by Portuguese funds through the Center for Research and Development in Mathematics and Applications (CIDMA), and the Portuguese Foundation for Science and Technology (FCT), within project UID/MAT/04106/2013.

Any comments or suggestions related to the material here contained are more than welcome, and may be submitted by post or by electronic mail to the authors:

Ricardo Almeida <ricardo. almeida@ua.pt> Center for Research and Development in Mathematics and Applications Department of Mathematics, University of Aveiro 3810-193 Aveiro, Portugal

Dina Tavares <dtavares@ipleiria.pt>

ESECS, Polytechnic Institute of Leiria

2410-272 Leiria, Portugal

Delfim F. M. Torres <delfim@ua.pt>

Center for Research and Development in Mathematics and Applications Department of Mathematics, University of Aveiro

3810-193 Aveiro, Portugal 



\section{Contents}

1 Fractional calculus ............................ 1

1.1 Historical perspective .......................... 1

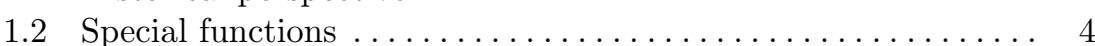

1.3 Fractional integrals and derivatives $\ldots \ldots \ldots \ldots \ldots \ldots \ldots \ldots$

1.3.1 Classical operators ....................... 6

1.3.2 Some properties of the Caputo derivative ........... 9

1.3.3 Combined Caputo derivative .................. 11

1.3.4 Variable-order operators ...................... 12

1.3.5 Generalized fractional operators .............. 14

1.3.6 Integration by parts $\ldots \ldots \ldots \ldots \ldots \ldots \ldots \ldots \ldots$

2 The calculus of variations ......................... 21

2.1 The classical calculus of variations ................... 21

2.1.1 Euler-Lagrange equations . ................. 23

2.1.2 Problems with variable endpoints............... 24

2.1.3 Constrained variational problems ............... 25

2.2 Fractional calculus of variations $\ldots \ldots \ldots \ldots \ldots \ldots \ldots \ldots . \ldots \ldots$

2.2.1 Fractional Euler-Lagrange equations ............. 27

2.2.2 Fractional variational problems of variable-order...... 29

3 Expansion formulas for fractional derivatives ........... 35

3.1 Caputo-type fractional operators of variable-order ........ 35

3.1.1 Caputo derivatives for functions of one variable ...... 36

3.1.2 Caputo derivatives for functions of several variables.... 42

3.2 Numerical approximations . . . . . . . . . . . . . . . . 45

$3.3 \quad$ Example ................................. 54

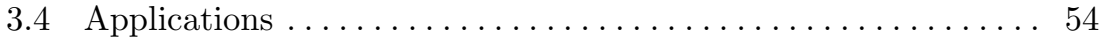

3.4.1 A time-fractional diffusion equation........... 55

3.4.2 A fractional partial differential equation in fluid mechanics ............................ 58 
XVI Contents

4 The fractional calculus of variations $\ldots \ldots \ldots \ldots \ldots \ldots \ldots$

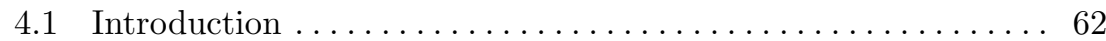

4.1.1 Combined operators of variable-order ............ 62

4.1.2 Generalized fractional integration by parts . . . . . . . 66

$4.2 \quad$ Fundamental variational problem ................... 68

4.2.1 Necessary optimality conditions . . . . . . . . . . . . 68

$4.2 .2 \quad$ Particular cases . . . . . . . . . . . . . . . . . . . . . 73

4.2 .3 Examples ............................. 74

$4.3 \quad$ Higher-order variational problems $\ldots \ldots \ldots \ldots \ldots \ldots \ldots \ldots . \ldots \ldots$

4.3.1 Necessary optimality conditions .............. 75

4.3.2 Example ........................... 82

4.4 Variational problems with time delay $\ldots \ldots \ldots \ldots \ldots \ldots \ldots . \ldots . \ldots 2$

4.4.1 Necessary optimality conditions ............... 83

4.4 .2 Example ............................. 86

4.5 Isoperimetric problems ...................... 86

4.5.1 Necessary optimality conditions I . . . . . . . . . . 87

4.5.2 Necessary optimality conditions II . . . . . . . . . . . . 92

4.5.3 Example ................................ 94

4.6 Variational problems with holonomic constraints .......... 95

4.6.1 Necessary optimality conditions . . . . . . . . . . 96

4.6.2 Example .............................. 100

4.7 Fractional variational Herglotz problem . . . . . . . . . . . . . 100

4.7.1 Fundamental problem of Herglotz . . . . . . . . . . . . 101

4.7.2 Several independent variables . . . . . . . . . . . . . . 104

4.7.3 Examples ............................ 108

\section{Appendix}

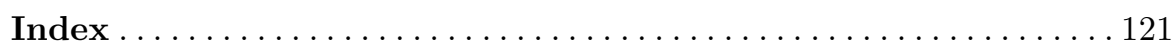




\section{Fractional calculus}

In this chapter, a brief introduction to the theory of fractional calculus is presented. We start with a historical perspective of the theory, with a strong connection with the development of classical calculus (Section 1.1). Then, in Section 1.2, we review some definitions and properties about a few special functions that will be needed. We end with a review on fractional integrals and fractional derivatives of noninteger order and with some formulas of integration by parts, involving fractional operators (Section 1.3).

The content of this chapter can be found in some classical books on fractional calculus, for example Almeida. Pooseh and Torres, 2015; Kilbas, Srivastava and Trujillo, 2006; Malinowska and Torres, 2012a; Podlubny, 1999; Samko, Kilbas and Marichev, 1993).

\subsection{Historical perspective}

Fractional Calculus (FC) is considered as a branch of mathematical analysis which deals with the investigation and applications of integrals and derivatives of arbitrary order. Therefore, FC is an extension of the integer-order calculus that considers integrals and derivatives of any real or complex order (Kilbas. Srivastava and Truiillo, 2006; Samko. Kilbas and Marichev, 1993), i.e., unify and generalize the notions of integer-order differentiation and $n$ fold integration.

FC was born in 1695 with a letter that L'Hôpital wrote to Leibniz, where the derivative of order $1 / 2$ is suggested (Oldham and Spanier, 1974). After Leibniz had introduced in his publications the notation for the $n$th derivative of a function $y$,

$$
\frac{d^{n} y}{d x^{n}},
$$

L'Hôpital wrote a letter to Leibniz to ask him about the possibility of a derivative of integer order to be extended in order to have a meaning when the order is a fraction: "What if $n$ be 1/2?" (Ross, 1977). In his answer, dated 
on 30 September 1695, Leibniz replied that "This is an apparent paradox from which, one day, useful consequences will be drawn" and, today, we know it is truth. Then, Leibniz still wrote about derivatives of general order and in 1730, Euler investigated the result of the derivative when the order $n$ is a fraction. But, only in 1819, with Lacroix, appeared the first definition of fractional derivative based on the expression for the $n$th derivative of the power function. Considering $y=x^{m}$, with $m$ a positive integer, Lacroix developed the $n$th derivative

$$
\frac{d^{n} y}{d x^{n}}=\frac{m !}{(m-n) !} x^{m-n}, \quad m \geq n,
$$

and using the definition of Gamma function, for the generalized factorial, he got

$$
\frac{d^{n} y}{d x^{n}}=\frac{\Gamma(m+1)}{\Gamma(m-n+1)} x^{m-n} .
$$

Lacroix also studied the following example, for $n=\frac{1}{2}$ and $m=1$ :

$$
\frac{d^{1 / 2} x}{d x^{1 / 2}}=\frac{\Gamma(2)}{\Gamma(3 / 2)} x^{\frac{1}{2}}=\frac{2 \sqrt{x}}{\sqrt{\pi}} .
$$

Since then, many mathematicians, like Fourier, Abel, Riemann, Liouville, among others, contributed to the development of this subject. One of the first applications of fractional calculus appear in 1823 by Niels Abel, through the solution of an integral equation of the form

$$
\int_{0}^{t}(t-\tau)^{-\alpha} x(\tau) d \tau=k,
$$

used in the formulation of the Tautochrone problem (Abel, 1923; Ross, 1977).

Different forms of fractional operators have been introduced along time, like the Riemann-Liouville, the Grünwald-Letnikov, the Weyl, the Caputo, the Marchaud or the Hadamard fractional derivatives (Kilbas. Srivastava and Trujillo, 2006; Oldham and Spanier, 1974; Oliveira and Machado, 2014; Podlubny, 1999). The first approach is the Riemann-Liouville, which is based on iterating the classical integral operator $n$ times and then considering the Cauchy's formula where $n$ ! is replaced by the Gamma function and hence the fractional integral of noninteger order is defined. Then, using this operator, some of the fractional derivatives mentioned above are defined.

During three centuries, FC was developed but as a pure theoretical subject of mathematics. In recent times, FC had an increasing of importance due to its applications in various fields, not only in mathematics, but also in physics, mechanics, engineering, chemistry, biology, finance, and others areas of science (Herrmann, 2013; Hilfer, 2000; Li and Liu, 2016; Mainardi, 2010; Odzijewicz. Malinowska and Torres, 2013c; Pinto and Carvalho, 2014; 
Sierociuk et al., 2015; Sun. Chen and Chen, 2009). In some of these applications, many real world phenomena are better described by noninteger order derivatives, if we compare with the usual integer-order calculus. In fact, fractional order derivatives have unique characteristics that may model certain dynamics more efficiently. Firstly, we can consider any real order for the derivatives, and thus we are not restricted to integer order derivatives only; secondly, they are nonlocal operators, in opposite to the usual derivatives, thus containing memory. With the memory property, one can take into account the past of the processes. Signal processing, modeling and control are some areas that have been the object of more intensive publishing in the last decades.

In most applications of the FC, the order of the derivative is assumed to be fixed along the process, that is, when determining what is the order $\alpha>0$ such that the solution of the fractional differential equation $D^{\alpha} y(t)=f(t, y(t))$ better approaches the experimental data, we consider the order $\alpha$ to be a fixed constant. Of course, this may not be the best option, since trajectories are a dynamic process, and the order may vary. More interesting possibilities arise when one considers the order $\alpha$ of the fractional integrals and derivatives not constant during the process but to be a function $\alpha(t)$, depending on time. Then, we may seek what is the best function $\alpha(\cdot)$ such that the variable-order fractional differential equation $D^{\alpha(t)} y(t)=f(t, y(t))$ better describes the process under study. This approach is very recent. One such fractional calculus of variable-order was introduced in (Samko and Ross, 1993). Afterwards, several mathematicians obtained important results about variable-order fractional calculus, and some applications appeared, like in mechanics, in the modeling of linear and nonlinear viscoelasticity oscillators and in other phenomena where the order of the derivative varies with time. See, for instance, (Almeida and Torres, 2013; Atanacković and Pilipović, 2011; Coimbra, 2003; Odzijewicz. Malinowska and Torres, 2013a; Ramirez and Coimbra, 2011; Samko, 1995; Sheng et al., 2011).

The most common fractional operators considered in the literature take into account the past of the process. They are usually called left fractional operators. But in some cases we may be also interested in the future of the process, and the computation of $\alpha(t)$ to be influenced by it. In that case, right fractional derivatives are then considered. Recently, in some works, the main goal is to develop a theory where both fractional operators are taken into account. For that, some combined fractional operators are introduced, like the symmetric fractional derivative, the Riesz fractional integral and derivative, the Riesz-Caputo fractional derivative and the combined Caputo fractional derivative that consists in a linear combination of the left and right fractional operators. For studies with fixed fractional order, see (Klimek, 2001; Malinowska and Torres, 2011, 2012a, b).

Due to the growing number of applications of fractional calculus in science and engineering, numerical approaches are being developed to provide tools for solving such problems. At present, there are already vast studies on numerical 
approximate formulas Kumar. Pandey and Sharma, 2017; Li. Chen and Ye, 2011). For example, for numerical modeling of time fractional diffusion equations, we refer the reader to (Fu, Chen and Yang, 2013).

\subsection{Special functions}

Before introducing the basic facts on fractional operators, we recall four types of functions that are important in Fractional Calculus: the Gamma, Psi, Beta and Mittag-Leffler functions. Some properties of these functions are also recalled.

Definition 1 The Euler Gamma function is an extension of the factorial function to real numbers, and it is defined by

$$
\Gamma(t)=\int_{0}^{\infty} \tau^{t-1} \exp (-\tau) d \tau, \quad t>0 .
$$

For example, $\Gamma(1)=1, \Gamma(2)=1$ and $\Gamma(3 / 2)=\frac{\sqrt{\pi}}{2}$. For positive integers $n$, we get $\Gamma(n)=(n-1)$ !. We mention that other definitions for the Gamma function exist, and it is possible to define it for complex numbers, except for the non-positive integers.

The Gamma function is considered the most important Eulerian function used in fractional calculus, because it appears in almost every fractional integral and derivative definitions. A basic but fundamental property of $\Gamma$, that we will use later, is obtained using integration by parts:

$$
\Gamma(t+1)=t \Gamma(t) .
$$

Definition 2 The Psi function is the derivative of the logarithm of the Gamma function:

$$
\Psi(t)=\frac{d}{d t} \ln (\Gamma(t))=\frac{\Gamma^{\prime}(t)}{\Gamma(t)} .
$$

The follow function is used sometimes for convenience to replace a combination of Gamma functions. It is important in FC because it shares a form that is similar to the fractional derivative or integral of many functions, particularly power functions.

Definition 3 The Beta function B is defined by

$$
B(t, u)=\int_{0}^{1} s^{t-1}(1-s)^{u-1} d s, \quad t, u>0 .
$$

This function satisfies an important property:

$$
B(t, u)=\frac{\Gamma(t) \Gamma(u)}{\Gamma(t+u)} .
$$


With this property, it is obvious that the Beta function is symmetric, i.e.,

$$
B(t, u)=B(u, t) .
$$

The next function is a direct generalization of the exponential series and it was defined by the mathematician Mittag-Leffler in 1903 (Podlubny, 1999).

Definition 4 Let $\alpha>0$. The function $E_{\alpha}$ defined by

$$
E_{\alpha}(t)=\sum_{k=0}^{\infty} \frac{t^{k}}{\Gamma(\alpha k+1)}, \quad t \in \mathbb{R},
$$

is called the one parameter Mittag-Leffler function.

For $\alpha=1$, this function coincides with the series expansion of $e^{t}$, i.e.,

$$
E_{1}(t)=\sum_{k=0}^{\infty} \frac{t^{k}}{\Gamma(k+1)}=\sum_{k=0}^{\infty} \frac{t^{k}}{k !}=e^{t} .
$$

While linear ordinary differential equations present in general the exponential function as a solution, the Mitttag-Leffler function occurs naturally in the solution of fractional order differential equations (Kilbas, Srivastava and Trujillo, 2006). For this reason, in recent times, the Mittag-Leffler function has become an important function in the theory of the fractional calculus and its applications.

It is also common to represent the Mittag-Leffler function in two arguments. This generalization of Mittag-Leffler function was studied by Wiman in 1905 (Mainardi, 2010).

Definition 5 The two-parameter function of the Mittag-Leffler type with parameters $\alpha, \beta>0$ is defined by

$$
E_{\alpha, \beta}(t)=\sum_{k=0}^{\infty} \frac{t^{k}}{\Gamma(\alpha k+\beta)}, \quad t \in \mathbb{R} .
$$

If $\beta=1$, this function coincides with the classical Mittag-Leffler function, i.e., $E_{\alpha, 1}(t)=E_{\alpha}(t)$.

\subsection{Fractional integrals and derivatives}

In this section, we recall some definitions of fractional integral and fractional differential operators, that includes all we use throughout this book. In the end, we present some integration by parts formulas because they have a crucial role in deriving Euler-Lagrange equations. 


\subsubsection{Classical operators}

As it was seen in Section 1.1, there are more than one way to generalize integerorder operations to the non-integer case. Here, we present several definitions and properties about fractional operators, omitting some details about the conditions that ensure the existence of such fractional operators.

In general, the fractional derivatives are defined using fractional integrals. We present only two fractional integrals operators, but there are several known forms of the fractional integrals.

Let $x:[a, b] \rightarrow \mathbb{R}$ be an integrable function and $\alpha>0$ a real number.

Starting with Cauchy's formula for a $n$-fold iterated integral, given by

$$
\begin{aligned}
{ }_{a} I_{t}^{n} x(t) & =\int_{a}^{t} d \tau_{1} \int_{a}^{\tau_{1}} d \tau_{2} \cdots \int_{a}^{\tau_{n-1}} x\left(\tau_{n}\right) d \tau_{n} \\
& =\frac{1}{(n-1) !} \int_{a}^{t}(t-\tau)^{n-1} x(\tau) d \tau,
\end{aligned}
$$

where $n \in \mathbb{N}$, Liouville and Riemann defined fractional integration, generalizing equation (1.2) to noninteger values of $n$ and using the definition of Gamma function $\Gamma$. With this, we introduce two important concepts: the left and the right Riemann-Liouville fractional integrals.

Definition 6 We define the left and right Riemann-Liouville fractional integrals of order $\alpha$, respectively, by

$$
{ }_{a} I_{t}^{\alpha} x(t)=\frac{1}{\Gamma(\alpha)} \int_{a}^{t}(t-\tau)^{\alpha-1} x(\tau) d \tau, \quad t>a
$$

and

$$
{ }_{t} I_{b}{ }^{\alpha} x(t)=\frac{1}{\Gamma(\alpha)} \int_{t}^{b}(\tau-t)^{\alpha-1} x(\tau) d \tau, \quad t<b .
$$

The constants $a$ and $b$ determine, respectively, the lower and upper boundary of the integral domain. Additionally, if $x$ is a continuous function, as $\alpha \rightarrow 0,{ }_{a} I_{t}{ }^{\alpha}={ }_{t} I_{b}{ }^{\alpha}=I$, with $I$ the identity operator, i.e., ${ }_{a} I_{t}{ }^{\alpha} x(t)=$ ${ }_{t} I_{b}{ }^{\alpha} x(t)=x(t)$.

We present the second fractional integral operator, introduced by $\mathrm{J}$. Hadamard in 1892 (Kilbas, Srivastava and Trujillo, 2006).

Definition 7 We define the left and right Hadamard fractional integrals of order $\alpha$, respectively, by

$$
{ }_{a} J_{t}^{\alpha} x(t)=\frac{1}{\Gamma(\alpha)} \int_{a}^{t}\left(\ln \frac{t}{\tau}\right)^{\alpha-1} \frac{x(\tau)}{\tau} d \tau, \quad t>a
$$

and

$$
{ }_{t} J_{b}^{\alpha} x(t)=\frac{1}{\Gamma(\alpha)} \int_{t}^{b}\left(\ln \frac{\tau}{t}\right)^{\alpha-1} \frac{x(\tau)}{\tau} d \tau, \quad t<b .
$$


The three most frequently used definitions for fractional derivatives are: the Grünwald-Letnikov, the Riemann-Liouville and the Caputo fractional derivatives (Oldham and Spanier, 1974; Podlubny, 1999). Other definitions were introduced by others mathematicians, as for instance Weyl, Fourier, Cauchy, Abel.

Let $x \in A C([a, b] ; \mathbb{R})$ be an absolutely continuous functions on the interval $[a, b]$, and $\alpha$ a positive real number. Using Definition 6 of Riemann-Liouville fractional integrals, we define the left and the right Riemann-Liouville and Caputo derivatives as follows.

Definition 8 We define the left and right Riemann-Liouville fractional derivatives of order $\alpha>0$, respectively, by

$$
\begin{aligned}
{ }_{a} D_{t}^{\alpha} x(t) & =\left(\frac{d}{d t}\right)^{n}{ }_{a} I_{t}^{n-\alpha} x(t) \\
& =\frac{1}{\Gamma(n-\alpha)}\left(\frac{d}{d t}\right)^{n} \int_{a}^{t}(t-\tau)^{n-\alpha-1} x(\tau) d \tau, \quad t>a
\end{aligned}
$$

and

$$
\begin{aligned}
{ }_{t} D_{b}^{\alpha} x(t) & =\left(-\frac{d}{d t}\right)^{n}{ }_{t} I_{b}^{n-\alpha} x(t) \\
& =\frac{(-1)^{n}}{\Gamma(n-\alpha)}\left(\frac{d}{d t}\right)^{n} \int_{t}^{b}(\tau-t)^{n-\alpha-1} x(\tau) d \tau, \quad t<b,
\end{aligned}
$$

where $n=[\alpha]+1$

The following definition was introduced in (Caputo, 1967). The Caputo fractional derivatives, in general, are more applicable and interesting in fields like physics and engineering, for its properties like the initial conditions.

Definition 9 We define the left and right Caputo fractional derivatives of order $\alpha$, respectively, by

$$
\begin{aligned}
{ }_{a}^{C} D_{t}^{\alpha} x(t) & ={ }_{a} I_{t}^{n-\alpha}\left(\frac{d}{d t}\right)^{n} x(t) \\
& =\frac{1}{\Gamma(n-\alpha)} \int_{a}^{t}(t-\tau)^{n-\alpha-1} x^{(n)}(\tau) d \tau, \quad t>a
\end{aligned}
$$

and

$$
\begin{aligned}
{ }_{t}^{C} D_{b}^{\alpha} x(t) & ={ }_{t} I_{b}^{n-\alpha}\left(-\frac{d}{d t}\right)^{n} x(t) \\
& =\frac{(-1)^{n}}{\Gamma(n-\alpha)} \int_{t}^{b}(\tau-t)^{n-\alpha-1} x^{(n)}(\tau) d \tau, \quad t<b,
\end{aligned}
$$

where $n=[\alpha]+1$ if $\alpha \notin \mathbb{N}$ and $n=\alpha$ if $\alpha \in \mathbb{N}$. 
Obviously, the above defined operators are linear. From these definitions, it is clear that the Caputo fractional derivative of a constant $C$ is zero, which is false when we consider the Riemann-Liouville fractional derivative. If $x(t)=$ $C$, with $C$ a constant, then we get

$$
{ }_{a}^{C} D_{t}^{\alpha} x(t)={ }_{t}^{C} D_{b}^{\alpha} x(t)=0
$$

and

$$
{ }_{a} D_{t}^{\alpha} x(t)=\frac{C(t-a)^{-\alpha}}{\Gamma(1-\alpha)}, \quad{ }_{t} D_{b}^{\alpha} x(t)=\frac{C(b-t)^{-\alpha}}{\Gamma(1-\alpha)} .
$$

For this reason, in some applications, Caputo fractional derivatives seem to be more natural than the Riemann-Liouville fractional derivatives.

Remark 1 If $\alpha$ goes to $n^{-}$, with $n \in \mathbb{N}$, then the fractional operators introduced above coincide with the standard derivatives:

$$
{ }_{a} D_{t}^{\alpha}={ }_{a}^{C} D_{t}^{\alpha}=\left(\frac{d}{d t}\right)^{n}
$$

and

$$
{ }_{t} D_{b}^{\alpha}={ }_{t}^{C} D_{b}^{\alpha}=\left(-\frac{d}{d t}\right)^{n} .
$$

The Riemann-Liouville fractional integral and differential operators of order $\alpha>0$ of power functions return power functions, as we can see below.

Lemma 2 Let $x$ be the power function $x(t)=(t-a)^{\gamma}$. Then, we have

$$
{ }_{a} I_{t}^{\alpha} x(t)=\frac{\Gamma(\gamma+1)}{\Gamma(\gamma+\alpha+1)}(t-a)^{\gamma+\alpha}, \quad \gamma>-1
$$

and

$$
{ }_{a} D_{t}^{\alpha} x(t)=\frac{\Gamma(\gamma+1)}{\Gamma(\gamma-\alpha+1)}(t-a)^{\gamma-\alpha}, \quad \gamma>-1 .
$$

In particular, if we consider $\gamma=1, a=0$ and $\alpha=1 / 2$, then the left Riemann-Liouville fractional derivative of $x(t)=t$ is $\frac{2 \sqrt{t}}{\sqrt{\pi}}$, the same result (1.1) as Lacroix obtained in 1819.

Grünwald and Letnikov, respectively in 1867 and 1868, returned to the original sources and started the formulation by the fundamental definition of a derivative, as a limit,

$$
x^{(1)}(t)=\lim _{h \rightarrow 0} \frac{x(t+h)-x(t)}{h}
$$

and considering the iteration at the $n$th order derivative formula:

$$
x^{(n)}(t)=\lim _{h \rightarrow 0} \frac{1}{h^{n}} \sum_{k=0}^{n}(-1)^{k}\left(\begin{array}{l}
n \\
k
\end{array}\right) x(t-k h), \quad n \in \mathbb{N},
$$


where $\left(\begin{array}{l}n \\ k\end{array}\right)=\frac{n(n-1)(n-2) \ldots(n-k+1)}{k !}$, with $n, k \in \mathbb{N}$, the usual notation for the binomial coefficients. The Grünwald-Letnikov definition of fractional derivative consists in a generalization of (1.3) to derivatives of arbitrary order $\alpha$ (Podlubnv, 1999).

Definition 10 The $\alpha$ th order Grünwald-Letnikov fractional derivative of function $x$ is given by

$$
{ }_{a}^{G L} D_{t}^{\alpha} x(t)=\lim _{h \longrightarrow 0} h^{-\alpha} \sum_{k=0}^{n}(-1)^{k}\left(\begin{array}{l}
\alpha \\
k
\end{array}\right) x(t-k h),
$$

where $\left(\begin{array}{l}\alpha \\ k\end{array}\right)=\frac{\Gamma(\alpha+1)}{k ! \Gamma(\alpha-k+1)}$ and $n h=t-a$.

Lemma 3 Let $x$ be the power function $x(t)=(t-a)^{\gamma}$, where $\gamma$ is a real number. Then, for $0<\alpha<1$ and $\gamma>0$, we have

$$
{ }_{a}^{G L} D_{t}^{\alpha} x(t)=\frac{\Gamma(\gamma+1)}{\Gamma(\gamma-\alpha+1)}(t-a)^{\gamma-\alpha} .
$$

Definition 11 We define the left and right Hadamard fractional derivatives of order $\alpha$, respectively, by

$$
{ }_{a} \mathrm{D}_{t}^{\alpha} x(t)=\left(t \frac{d}{d t}\right)^{n} \frac{1}{\Gamma(n-\alpha)} \int_{a}^{t}\left(\ln \frac{t}{\tau}\right)^{n-\alpha-1} \frac{x(\tau)}{\tau} d \tau
$$

and

$$
{ }_{t} \mathrm{D}_{b}^{\alpha} x(t)=\left(-t \frac{d}{d t}\right)^{n} \frac{1}{\Gamma(n-\alpha)} \int_{t}^{b}\left(\ln \frac{\tau}{t}\right)^{n-\alpha-1} \frac{x(\tau)}{\tau} d \tau,
$$

for all $t \in(a, b)$, where $n=[\alpha]+1$.

Observe that, for all types of derivative operators, if variable $t$ is the timevariable, the left fractional derivative of $x$ is interpreted as a past state of the process, while the right fractional derivative of $x$ is interpreted as a future state of the process.

\subsubsection{Some properties of the Caputo derivative}

For $\alpha>0$ and $x \in A C([a, b] ; \mathbb{R})$, the Riemann-Liouville and Caputo derivatives are related by the following formulas (Kilbas. Srivastava and Truiillo, 2006):

$$
{ }_{a}^{C} D_{t}^{\alpha} x(t)={ }_{a} D_{t}^{\alpha}\left[x(t)-\sum_{k=0}^{n-1} \frac{x^{(k)}(a)(t-a)^{k}}{k !}\right]
$$

and

$$
{ }_{t}^{C} D_{b}^{\alpha} x(t)={ }_{t} D_{b}^{\alpha}\left[x(t)-\sum_{k=0}^{n-1} \frac{x^{(k)}(b)(b-t)^{k}}{k !}\right],
$$


where $n=[\alpha]+1$ if $\alpha \notin \mathbb{N}$ and $n=\alpha$ if $\alpha \in \mathbb{N}$.

In particular, when $\alpha \in(0,1)$, the relations (1.4) and (1.5) take the form:

$$
\begin{aligned}
{ }_{a}^{C} D_{t}^{\alpha} x(t) & ={ }_{a} D_{t}^{\alpha}(x(t)-x(a)) \\
& ={ }_{a} D_{t}^{\alpha} x(t)-\frac{x(a)}{\Gamma(1-\alpha)}(t-a)^{-\alpha}
\end{aligned}
$$

and

$$
\begin{aligned}
{ }_{t}^{C} D_{b}^{\alpha} x(t) & ={ }_{t} D_{b}^{\alpha}(x(t)-x(b)) \\
& ={ }_{t} D_{b}^{\alpha} x(t)-\frac{x(b)}{\Gamma(1-\alpha)}(b-t)^{-\alpha} .
\end{aligned}
$$

It follows from (1.6) and (1.7) that the left Riemann-Liouville derivative equals the left Caputo fractional derivative in the case $x(a)=0$ and the analogue holds for the right derivatives under the assumption $x(b)=0$.

In Theorem 4, we see that the Caputo fractional derivatives provides a left inverse operator to the Riemann-Liouville fractional integration (cf. Lemma 2.21 in (Kilbas, Srivastava and Trujillo, 2006)).

Theorem 4 Let $\alpha>0$ and let $x \in C\left([a, b] ; \mathbb{R}^{n}\right)$. For Caputo fractional operators, the next rules hold:

$$
{ }_{a}^{C} D_{t}^{\alpha}{ }_{a} I_{t}^{\alpha} x(t)=x(t)
$$

and

$$
{ }_{t}^{C} D_{b t}^{\alpha} I_{b}^{\alpha} x(t)=x(t) .
$$

The next statement characterizes the composition of the Riemann-Liouville fractional integration operators with the Caputo fractional differentiation operators (cf. Lemma 2.22 in (Kilbas, Srivastava and Trujillo, 2006)).

Theorem 5 Let $\alpha>0$. If $x \in A C^{n}([a, b] ; \mathbb{R})$, then

$$
{ }_{a} I_{t}^{\alpha}{ }_{a} D_{t}^{\alpha} x(t)=x(t)-\sum_{k=0}^{n-1} \frac{x^{(k)}(a)}{k !}(t-a)^{k}
$$

and

$$
{ }_{t} I_{b \quad t}^{\alpha C} D_{b}^{\alpha} x(t)=x(t)-\sum_{k=0}^{n-1} \frac{(-1)^{k} x^{(k)}(b)}{k !}(b-t)^{k},
$$

with $n=[\alpha]+1$ if $\alpha \notin \mathbb{N}$ and $n=\alpha$ if $\alpha \in \mathbb{N}$.

In particular, when $\alpha \in(0,1)$, then

$$
{ }_{a} I_{t}^{\alpha C}{ }_{a}^{C} D_{t}^{\alpha} x(t)=x(t)-x(a) \quad \text { and }{ }_{t} I_{b t}^{\alpha}{ }_{t}^{C} D_{b}^{\alpha} x(t)=x(t)-x(b) .
$$

Similarly to the Riemann-Liouville fractional derivative, the Caputo fractional derivative of a power function yields a power function of the same form. 
Lemma 6 Let $\alpha>0$. Then, the following relations hold:

$$
{ }_{a}^{C} D_{t}^{\alpha}(t-a)^{\gamma}=\frac{\Gamma(\gamma+1)}{\Gamma(\gamma-\alpha+1)}(t-a)^{\gamma-\alpha}, \quad \gamma>n-1
$$

and

$$
{ }_{t}^{C} D_{b}^{\alpha}(b-t)^{\gamma}=\frac{\Gamma(\gamma+1)}{\Gamma(\gamma-\alpha+1)}(b-t)^{\gamma-\alpha}, \quad \gamma>n-1,
$$

with $n=[\alpha]+1$ if $\alpha \notin \mathbb{N}$ and $n=\alpha$ if $\alpha \in \mathbb{N}$.

\subsubsection{Combined Caputo derivative}

In this section, we introduce a special operator for our work, the combined Caputo fractional derivative. We extend the notion of the Caputo fractional derivative to the fractional derivative ${ }^{C} D_{\gamma}^{\alpha, \beta}$, that involves the left and the right Caputo fractional derivative, i.e., it combines the past and the future of the process into one single operator.

This operator was introduced in (Malinowska and Torres, 2010), motivated by the ideia of the symmetric fractional derivative introduced in (Klimek, 2001):

Definition 12 Considering the left and right Riemann-Liouville derivatives, the symmetric fractional derivative is given by

$$
{ }_{a} D_{b}^{\alpha} x(t)=\frac{1}{2}\left[{ }_{a} D_{t}^{\alpha} x(t)+{ }_{t} D_{b}^{\alpha} x(t)\right] .
$$

Other combined operators were studied. For example, we have the Riesz and the Riesz-Caputo operators (Malinowska and Torres, 2012a).

Definition 13 Let $x:[a, b] \rightarrow \mathbb{R}$ be a function of class $C^{1}$ and $\alpha \in(0,1)$. For $t \in[a, b]$, the Riesz fractional integral of order $\alpha$, is defined by

$$
\begin{aligned}
{ }_{a}^{R} I_{b}^{\alpha} x(t) & =\frac{1}{2 \Gamma(\alpha)} \int_{a}^{b}|t-\tau|^{\alpha-1} x(\tau) d \tau \\
& =\frac{1}{2}\left[{ }_{a} I_{t}^{\alpha} x(t)+{ }_{t} I_{b}^{\alpha} x(t)\right],
\end{aligned}
$$

and the Riesz fractional derivative of order $\alpha$, is defined by

$$
\begin{aligned}
{ }_{a}^{R} D_{b}^{\alpha} x(t) & =\frac{1}{\Gamma(1-\alpha)} \frac{d}{d t} \int_{a}^{b}|t-\tau|^{-\alpha} x(\tau) d \tau \\
& =\frac{1}{2}\left[{ }_{a} D_{t}^{\alpha} x(t)-{ }_{t} D_{b}^{\alpha} x(t)\right] .
\end{aligned}
$$

Definition 14 Considering the left and right Caputo derivatives, RieszCaputo fractional derivative is given by 


$$
\begin{aligned}
{ }_{a}^{R C} D_{b}^{\alpha} x(t) & =\frac{1}{\Gamma(1-\alpha)} \int_{a}^{b}|t-\tau|^{-\alpha} \frac{d}{d \tau} x(\tau) d \tau \\
& =\frac{1}{2}\left[{ }_{a}^{C} D_{t}^{\alpha} x(t)-{ }_{t}^{C} D_{b}^{\alpha} x(t)\right] .
\end{aligned}
$$

Remark 7 (Malinowska and Torres (2012a)) If $\alpha$ goes to 1 , then the fractional derivatives introduced above coincide with the standard derivative:

$$
{ }_{a}^{R} D_{b}^{\alpha}={ }_{a}^{R C} D_{b}^{\alpha}=\frac{d}{d t}
$$

Similarly to the last operator (1.9), the combined Caputo derivative is a convex combination of the left and the right Caputo fractional derivatives. But, in this operator, we also consider other coefficients for the convex combination besides $1 / 2$. Moreover, the orders $\alpha$ and $\beta$ of the left- and right-sided fractional derivatives can be different. Therefore, the combined Caputo derivative is a convex combination of the left Caputo fractional derivative of order $\alpha$ and the right Caputo fractional derivative of order $\beta$.

Definition 15 Let $\alpha, \beta \in(0,1)$ and $\gamma \in[0,1]$. The combined Caputo fractional derivative operator ${ }^{C} D_{\gamma}^{\alpha, \beta}$ is defined by

$$
{ }^{C} D_{\gamma}^{\alpha, \beta}=\gamma_{a}^{C} D_{t}^{\alpha}+(1-\gamma){ }_{t}^{C} D_{b}^{\beta},
$$

which acts on $x \in A C([a, b] ; \mathbb{R})$ in the following way:

$$
{ }^{C} D_{\gamma}^{\alpha, \beta} x(t)=\gamma_{a}^{C} D_{t}^{\alpha} x(t)+(1-\gamma){ }_{t}^{C} D_{b}^{\beta} x(t) .
$$

The operator (1.10) is obviously linear. Observe that

$$
{ }^{C} D_{0}^{\alpha, \beta}={ }_{t}^{C} D_{b}^{\beta} \quad \text { and } \quad{ }^{C} D_{1}^{\alpha, \beta}={ }_{a}^{C} D_{t}^{\alpha} .
$$

The symmetric fractional derivative and the Riesz fractional derivative are useful tools to describe some nonconservative models. But those types of differentiation do not seem suitable for all kinds of variational problems because they are based on the Riemann-Liouville fractional derivatives and therefore the possibility that admissible trajectories $x$ have continuous fractional derivatives imply that $x(a)=x(b)=0$ (Samko and Ross, 1993 ). For more details about the combined Caputo fractional derivative, see (Malinowska and Torres, 2011, 2012b, c; Odzijewicz. Malinowska and Torres, 2012b).

\subsubsection{Variable-order operators}

Very useful physical applications have given birth to the variable-order fractional calculus, for example in modeling mechanical behaviors $\mathrm{Fu}$. Chen and Yang, 2013; Sun et al., 2013). Nowadays, variable-order fractional calculus is particularly recognized as a useful and promising approach in the modelling of 
diffusion processes, in order to characterize time-dependent or concentrationdependent anomalous diffusion, or diffusion processes in inhomogeneous porous media (Sun et al., 2012).

Now, we present the fundamental notions of the fractional calculus of variable-order (Malinowska. Odziiewicz and Torres, 2015). We consider the fractional order of the derivative and of the integral to be a continuous function of two variables, $\alpha(\cdot, \cdot)$ with domain $[a, b]^{2}$, taking values on the open interval $(0,1)$. Let $x:[a, b] \rightarrow \mathbb{R}$ be a function.

First, we recall the generalization of fractional integrals for a variable-order $\alpha(\cdot, \cdot)$.

Definition 16 The left and right Riemann-Liouville fractional integrals of order $\alpha(\cdot, \cdot)$ are defined by

$$
{ }_{a} I_{t}^{\alpha(\cdot, \cdot)} x(t)=\int_{a}^{t} \frac{1}{\Gamma(\alpha(t, \tau))}(t-\tau)^{\alpha(t, \tau)-1} x(\tau) d \tau, \quad t>a
$$

and

$$
{ }_{t} I_{b}^{\alpha(\cdot, \cdot)} x(t)=\int_{t}^{b} \frac{1}{\Gamma(\alpha(\tau, t))}(\tau-t)^{\alpha(\tau, t)-1} x(\tau) d \tau, \quad t<b,
$$

respectively.

We remark that, in contrast to the fixed fractional order case, variableorder fractional integrals are not the inverse operation of the variable-order fractional derivatives.

For fractional derivatives, we consider two types: the Riemann-Liouville and the Caputo fractional derivatives.

Definition 17 The left and right Riemann-Liouville fractional derivatives of order $\alpha(\cdot, \cdot)$ are defined by

$$
\begin{aligned}
{ }_{a} D_{t}^{\alpha(\cdot, \cdot)} x(t) & =\frac{d}{d t}{ }_{a} I_{t}^{\alpha(\cdot, \cdot)} x(t) \\
& =\frac{d}{d t} \int_{a}^{t} \frac{1}{\Gamma(1-\alpha(t, \tau))}(t-\tau)^{-\alpha(t, \tau)} x(\tau) d \tau, \quad t>a
\end{aligned}
$$

and

$$
\begin{aligned}
{ }_{t} D_{b}^{\alpha(\cdot, \cdot)} x(t) & =-\frac{d}{d t} I_{b}^{\alpha(\cdot, \cdot)} x(t) \\
& =\frac{d}{d t} \int_{t}^{b} \frac{-1}{\Gamma(1-\alpha(\tau, t))}(\tau-t)^{-\alpha(\tau, t)} x(\tau) d \tau, \quad t<b
\end{aligned}
$$

respectively.

Lemma 8 gives a Riemann-Liouville variable-order fractional integral and fractional derivative for the power function $x(t)=(t-a)^{\gamma}$, where we assume that the fractional order depends only on the first variable: $\alpha(t, \tau):=\bar{\alpha}(t)$, where $\bar{\alpha}:[a, b] \rightarrow(0,1)$ is a given function. 
Lemma 8 Let $x$ be the power function $x(t)=(t-a)^{\gamma}$. Then, for $\gamma>-1$, we have

$$
{ }_{a} I_{t}^{\bar{\alpha}(t)} x(t)=\frac{\Gamma(\gamma+1)}{\Gamma(\gamma+\bar{\alpha}(t)+1)}(t-a)^{\gamma+\bar{\alpha}(t)}
$$

and

$$
\begin{aligned}
{ }_{a} D_{t}^{\bar{\alpha}(t)} x(t) & =\frac{\Gamma(\gamma+1)}{\Gamma(\gamma-\bar{\alpha}(t)+1)}(t-a)^{\gamma-\bar{\alpha}(t)} \\
& -\bar{\alpha}^{(1)}(t) \frac{\Gamma(\gamma+1)}{\Gamma(\gamma-\bar{\alpha}(t)+2)}(t-a)^{\gamma-\bar{\alpha}(t)+1} \\
& \times[\ln (t-a)-\Psi(\gamma-\bar{\alpha}(t)+2)+\Psi(1-\bar{\alpha}(t))] .
\end{aligned}
$$

Definition 18 The left and right Caputo fractional derivatives of order $\alpha(\cdot, \cdot)$ are defined by

$$
{ }_{a}^{C} D_{t}^{\alpha(\cdot, \cdot)} x(t)=\int_{a}^{t} \frac{1}{\Gamma(1-\alpha(t, \tau))}(t-\tau)^{-\alpha(t, \tau)} x^{(1)}(\tau) d \tau, \quad t>a
$$

and

$$
{ }_{t}^{C} D_{b}^{\alpha(\cdot, \cdot)} x(t)=\int_{t}^{b} \frac{-1}{\Gamma(1-\alpha(\tau, t))}(\tau-t)^{-\alpha(\tau, t)} x^{(1)}(\tau) d \tau, \quad t<b,
$$

respectively.

Of course, the fractional derivatives just defined are linear operators.

\subsubsection{Generalized fractional operators}

In this section, we present three definitions of one-dimensional generalized fractional operators that depend on a general kernel, studied by Odzijewicz, Malinowska and Torres (see e.g. (Malinowska. Odzijewicz and Torres, 2015; Odzijewicz. Malinowska and Torres, 2012a, 2013c), although (Agrawal, 2010) had introduced this generalized fractional operators).

Let $\Delta:=\left\{(t, \tau) \in \mathbb{R}^{2}: a \leq \tau<t \leq b\right\}$.

Definition 19 Let $k^{\alpha}$ be a function defined almost everywhere on $\Delta$ with values in $\mathbb{R}$. For all $x:[a, b] \rightarrow \mathbb{R}$, the generalized fractional integral operator $K_{P}$ is defined by

$$
K_{P}[x](t)=\lambda \int_{a}^{t} k^{\alpha}(t, \tau) x(\tau) d \tau+\mu \int_{t}^{b} k^{\alpha}(\tau, t) x(\tau) d \tau,
$$

with $P=\langle a, t, b, \lambda, \mu\rangle$, where $\lambda$ and $\mu$ are real numbers.

In particular, if we choose special cases for the kernel, we can obtain standard fractional operators or variable-order.

Remark 9 For special chosen kernels $k^{\alpha}$ and parameters $P$, the operator $K_{P}$ can be reduced to the classical or variable-order Riemann-Liouville fractional integrals: 
- Let $k^{\alpha}(t, \tau)=\frac{1}{\Gamma(\alpha)}(t-\tau)^{\alpha-1}$ and $0<\alpha<1$. If $P=\langle a, t, b, 1,0\rangle$, then

$$
K_{P}[x](t)=\frac{1}{\Gamma(\alpha)} \int_{a}^{t}(t-\tau)^{\alpha-1} x(\tau) d \tau=:{ }_{a} I_{t}^{\alpha}[x](t)
$$

is the left Riemann-Liouville fractional integral of order $\alpha$; if $P=\langle a, t, b, 0,1\rangle$, then

$$
K_{P}[x](t)=\frac{1}{\Gamma(\alpha)} \int_{t}^{b}(\tau-t)^{\alpha-1} x(\tau) d \tau={ }_{t} I_{b}^{\alpha}[x](t)
$$

is the right Riemann-Liouville fractional integral of order $\alpha$.

- If $k^{\alpha}(t, \tau)=\frac{1}{\Gamma(\alpha(t, \tau))}(t-\tau)^{\alpha(t, \tau)-1}$ and $P=\langle a, t, b, 1,0\rangle$, then

$$
K_{P}[x](t)=\int_{a}^{t} \frac{1}{\Gamma(\alpha(t, \tau))}(t-\tau)^{\alpha(t, \tau)-1} x(\tau) d \tau=:{ }_{a} I_{t}^{\alpha(\cdot, \cdot)}[x](t)
$$

is the left Riemann-Liouville fractional integral of variable-order $\alpha(\cdot, \cdot)$; if $P=\langle a, t, b, 0,1\rangle$, then

$$
K_{P}[x](t)=\int_{t}^{b} \frac{1}{\Gamma(\alpha(t, \tau))}(\tau-t)^{\alpha(t, \tau)-1} x(\tau) d \tau=:{ }_{t} I_{b}^{\alpha(\cdot, \cdot)}[x](t)
$$

is the right Riemann-Liouville fractional integral of variable-order $\alpha(\cdot, \cdot)$.

Some other fractional operators can be obtained with the generalized fractional integrals, for example, Hadamard, Riesz, Katugampola fractional operators (Agrawal, 2010; Malinowska, Odzijewicz and Torres, 2015).

The following two news operators, the generalized fractional RiemannLiouville and Caputo derivatives, are defined as a composition of classical derivatives and generalized fractional integrals.

Definition 20 The generalized fractional derivative of Riemann-Liouville type, denoted by $A_{P}$, is defined by

$$
A_{P}=\frac{d}{d t} \circ K_{P}
$$

Definition 21 The generalized fractional derivative of Caputo type, denoted by $B_{P}$, is defined by

$$
B_{P}=K_{P} \circ \frac{d}{d t} .
$$

Considering $k^{\alpha}(t, \tau)=\frac{1}{\Gamma(1-\alpha)}(t-\tau)^{-\alpha}$ with $0<\alpha<1$ and appropriate sets $P$, this two general kernel operators $A_{P}$ and $B_{P}$ can be reduced to the standard Riemann-Liouville and Caputo fractional derivatives, respectively (Malinowska, Odzijewicz and Torres, 2015). 


\subsubsection{Integration by parts}

In this section, we summarize formulas of integration by parts because they are important results to find necessary optimality conditions when dealing with variational problems.

First, we present the rule of fractional integration by parts for the Riemann-Liouville fractional integral.

Theorem 10 Let $0<\alpha<1, p \geq 1, q \geq 1$ and $1 / p+1 / q \leq 1+\alpha$. If $y \in$ $L_{p}([a, b] ; \mathbb{R})$ and $x \in L_{q}([a, b] ; \mathbb{R})$, then the following formula for integration by parts hold:

$$
\int_{a}^{b} y(t){ }_{a} I_{t}^{\alpha} x(t) d t=\int_{a}^{b} x(t)_{t} I_{b}^{\alpha} y(t) d t .
$$

For Caputo fractional derivatives, the integration by parts formulas are presented below (Almeida and Malinowska, 2013).

Theorem 11 Let $0<\alpha<1$. The following relations hold:

$$
\int_{a}^{b} y(t){ }_{a}^{C} D_{t}^{\alpha} x(t) d t=\int_{a}^{b} x(t){ }_{t} D_{b}^{\alpha} y(t) d t+\left[x(t){ }_{t} I_{b}^{1-\alpha} y(t)\right]_{t=a}^{t=b}
$$

and

$$
\int_{a}^{b} y(t)_{t}^{C} D_{b}^{\alpha} x(t) d t=\int_{a}^{b} x(t)_{a} D_{t}^{\alpha} y(t) d t-\left[x(t)_{a} I_{t}^{1-\alpha} y(t)\right]_{t=a}^{t=b} .
$$

When $\alpha \rightarrow 1$, we get ${ }_{a}^{C} D_{t}^{\alpha}={ }_{a} D_{t}^{\alpha}=\frac{d}{d t},{ }_{t}^{C} D_{b}^{\alpha}={ }_{t} D_{b}^{\alpha}=-\frac{d}{d t},{ }_{a} I_{t}^{\alpha}={ }_{t} I_{b}^{\alpha}=$ $I$, and formulas (1.16) and (1.17) give the classical formulas of integration by parts.

Then, we introduce the integration by parts formulas for variable-order fractional integrals (Odzijewicz, Malinowska and Torres, 2013b).

Theorem 12 Let $\frac{1}{n}<\alpha(t, \tau)<1$ for all $t, \tau \in[a, b]$ and a certain $n \in \mathbb{N}$ greater or equal than two, and $x, y \in C([a, b] ; \mathbb{R})$. Then the following formula for integration by parts hold:

$$
\int_{a}^{b} y(t)_{a} I_{t}^{\alpha(\cdot, \cdot)} x(t) d t=\int_{a}^{b} x(t)_{t} I_{b}^{\alpha(\cdot, \cdot)} y(t) d t .
$$

In the following theorem, we present the formulas involving the Caputo fractional derivative of variable-order. The theorem was proved in (Odzijewicz, Malinowska and Torres, 2013b) and gives a generalization of the standard fractional formulas of integration by parts for a constant $\alpha$.

Theorem 13 Let $0<\alpha(t, \tau)<1-\frac{1}{n}$ for all $t, \tau \in[a, b]$ and a certain $n \in \mathbb{N}$ greater or equal than two. If $x, y \in C^{1}([a, b] ; \mathbb{R})$, then the fractional integration by parts formulas 


$$
\int_{a}^{b} y(t){ }_{a}^{C} D_{t}^{\alpha(\cdot, \cdot)} x(t) d t=\int_{a}^{b} x(t){ }_{t} D_{b}^{\alpha(\cdot, \cdot)} y(t) d t+\left[x(t){ }_{t} I_{b}^{1-\alpha(\cdot, \cdot)} y(t)\right]_{t=a}^{t=b}
$$

and

$$
\int_{a}^{b} y(t){ }_{t}^{C} D_{b}^{\alpha(\cdot, \cdot)} x(t) d t=\int_{a}^{b} x(t){ }_{a} D_{t}^{\alpha(\cdot, \cdot)} y(t) d t-\left[x(t){ }_{a} I_{t}^{1-\alpha(\cdot, \cdot)} y(t)\right]_{t=a}^{t=b}
$$

hold.

This last theorem have an important role in this work to the proof of the generalized Euler-Lagrange equations.

In the end of this chapter, we present integration by parts formulas for generalized fractional operators (Malinowska, Odzijewicz and Torres, 2015). For that, we need the following definition:

Definition 22 Let $P=\langle a, t, b, \lambda, \mu\rangle$. We denote by $P^{*}$ the parameter set $P^{*}=\langle a, t, b, \mu, \lambda\rangle$. The parameter $P^{*}$ is called the dual of $P$.

Let $1<p<\infty$ and $q$ be the adjoint of $p$, that is $\frac{1}{n}+\frac{1}{q}=1$. A proof of the next result can be found in (Malinowska, Odzijewicz and Torres, 2015).

Theorem 14 Let $k \in L_{q}(\Delta ; \mathbb{R})$. Then the operator $K_{P^{*}}$ is a linear bounded operator from $L_{p}([a, b] ; \mathbb{R})$ to $L_{q}([a, b] ; \mathbb{R})$. Moreover, the following integration by parts formula holds:

$$
\int_{a}^{b} x(t) \cdot K_{P}[y](t) d t=\int_{a}^{b} y(t) \cdot K_{P^{*}}[x](t) d t
$$

for all $x, y \in L_{p}([a, b] ; \mathbb{R})$.

\section{References}

Abel NH (1823) Solution de quelques problèmes à l'aide d'intégrales définies. Mag. Naturv. 1(2):1-127

Agrawal OP (2010) Generalized variational problems and Euler-Lagrange equations. Comput. Math. Appl. 59(5):1852-1864

Almeida R, Malinowska AB (2013) Generalized transversality conditions in fractional calculus of variations. Commun. Nonlinear Sci. Numer. Simul. 18(3):443-452

Almeida R, Pooseh S, Torres DFM (2015) Computational Methods in the Fractional Calculus of Variations. Imperial College Press, London

Almeida R, Torres DFM (2013) An expansion formula with higher-order derivatives for fractional operators of variable order. The Scientific World Journal 2013 Art. ID 915437, 11 pp arXiv:1309.4899

Atanacković TM, Pilipovic S (2011) Hamilton's principle with variable order fractional derivatives. Fract. Calc. Appl. Anal. 14:94-109 
Caputo M (1967) Linear model of dissipation whose $Q$ is almost frequency independent-II. Geophys. J. R. Astr. Soc. 13:529-539

Coimbra CFM (2003) Mechanics with variable-order differential operators. Ann. Phys. 12(11-12):692-703

Fu Z-J, Chen W, Yang H-T (2013) Boundary particle method for Laplace transformed time fractional diffusion equations. J. Comput. Phys. 235:5266

Herrmann R (2013) Folded potentials in cluster physics-a comparison of Yukawa and Coulomb potentials with Riesz fractional integrals. J. Phys. A 46(40):405203, $12 \mathrm{pp}$

Hilfer R (2000) Applications of fractional calculus in physics. World Sci. Publishing, River Edge, NJ

Kilbas AA, Srivastava HM, Trujillo JJ (2006) Theory and Applications of Fractional Differential Equations. Elsevier, Amsterdam

Klimek M (2001) Fractional sequential mechanics - models with symmetric fractional derivative. Czechoslovak J. Phys. 51(12):1348-1354

Kumar K, Pandey R, Sharma S (2017) Comparative study of three numerical schemes for fractional integro-differential equations. J. Comput. Appl. Math. 315:287-302

Li CP, Chen A, Ye J (2011) Numerical approaches to fractional calculus and fractional ordinary differential equation. J. Comput. Phys. 230(9):33523368

Li G, Liu H (2016) Stability analysis and synchronization for a class of fractional-order neural networks. Entropy 18(55):13 pp

Mainardi F (2010) Fractional Calculus and Waves in Linear Viscoelasticity. Imp. Coll. Press, London

Malinowska AB, Odzijewicz T, Torres DFM (2015) Advanced Methods in the Fractional Calculus of Variations. Springer Briefs in Applied Sciences and Technology, Springer, Cham

Malinowska AB, Torres DFM (2010) Fractional variational calculus in terms of a combined Caputo derivative. Proceedings of FDA'10, The 4th IFAC Workshop on Fractional Differentiation and its Applications, Badajoz, Spain, October 18-20, 2010 (Eds: I. Podlubny, B. M. Vinagre Jara, YQ. Chen, V. Feliu Batlle, I. Tejado Balsera):Article no. FDA10-084, 6 pp. arXiv:1007.0743

Malinowska AB, Torres DFM (2011) Fractional calculus of variations for a combined Caputo derivative. Fract. Calc. Appl. Anal. 14(4):523-537 arXiv:1109.4664

Malinowska AB, Torres DFM (2012a) Introduction to the Fractional Calculus of Variations. Imp. Coll. Press, London

Malinowska AB, Torres DFM (2012b) Multiobjective fractional variational calculus in terms of a combined Caputo derivative. Appl. Math. Comput. 218(9):5099-5111 arXiv:1110.6666

Malinowska AB, Torres DFM (2012c) Towards a combined fractional mechanics and quantization. Fract. Calc. Appl. Anal. 15(3):407-417 arXiv: 1206.0864 
Odzijewicz T, Malinowska AB, Torres DFM (2012a) Fractional calculus of variations in terms of a generalized fractional integral with applications to physics. Abstr. Appl. Anal. 2012:Art. ID 871912, 24 pp arXiv: 1203.1961

Odzijewicz T, Malinowska AB, Torres DFM (2012b) Fractional variational calculus with classical and combined Caputo derivatives. Nonlinear Anal. 75(3):1507-1515 arXiv: 1101. 2932

Odzijewicz T, Malinowska AB, Torres DFM (2013a) Fractional variational calculus of variable order. in Advances in harmonic analysis and operator theory:291-301, Oper. Theory Adv. Appl., Birkhäuser/Springer Basel AG, Basel arXiv:1110.4141

Odzijewicz T, Malinowska AB, Torres DFM (2013b) Noether's theorem for fractional variational problems of variable order. Cent. Eur. J. Phys. 11(6):691-701 arXiv: 1303.4075

Odzijewicz T, Malinowska AB, Torres DFM (2013c) A generalized fractional calculus of variations. Control Cybernet. 42(2):443-458 arXiv: 1304.5282

Oldham KB, Spanier J (1974) The Fractional Calculus. Academic Press, New York

Oliveira EC, Machado JAT (2014) A Review of definitions for fractional derivatives and integral. Math. Probl. in Eng. 2014:238459, 6 pp

Pinto C, Carvalho ARM (2014) New findings on the dynamics of HIV and TB coinfection models. Appl. Math. Comput. 242:36-46

Podlubny I (1999) Fractional Differential Equations. Academic Press, San Diego, CA

Ramirez LES, Coimbra CFM (2011) On the variable order dynamics of the nonlinear wake caused by a sedimenting particle. Phys. D 240(13):11111118

Ross B (1977) The development of fractional calculus 1695-1900. Historia Mathematica 4:75-89

Samko SG (1995) Fractional integration and differentiation of variable order. Anal. Math. 21(3):213-236

Samko SG, Kilbas AA, Marichev OI (1993) Fractional Integrals and Derivatives. translated from the 1987 Russian original, Gordon and Breach, Yverdon

Samko SG, Ross B (1993) Integration and differentiation to a variable fractional order. Integral Transform. Spec. Funct. 1(4):277-300

Sheng H, Sun HG, Coopmans C, Chen YQ, Bohannan GW (2011) A physical experimental study of variable-order fractional integrator and differentiator. Eur. Phys. J. 193(1):93-104

Sierociuk D, Skovranek T, Macias M, Podlubny I, Petras I, Dzielinski A, Ziubinski P (2015) Diffusion process modeling by using fractional-order models. Appl. Math. Comput. 257(15):2-11

Sun HG, Chen W, Chen YQ (2009) Variable order fractional differential operators in anomalous diffusion modeling. Physica A. 388(21):4586-4592 
Sun H, Chen W, Li C, Chen Y (2012) Finite difference schemes for variableorder time fractional diffusion equation. Internat. J. Bifur. Chaos Appl. Sci. Engrg. 22(4):1250085, $16 \mathrm{pp}$

Sun H, Hu S, Chen Y, Chen W, Yu Z (2013) A dynamic-order fractional dynamic system. Chinese Phys. Lett. 30(4):046601, 4 pp 


\section{The calculus of variations}

As part of this book is devoted to the fractional calculus of variations, in this chapter we introduce the basic concepts about the classical calculus of variations and the fractional calculus of variations. The study of fractional problems of the calculus of variations and respective Euler-Lagrange type equations is a subject of current strong research.

In Section 2.1, we introduce some concepts and important results from the classical theory. Afterwards, in Section 2.2, we start with a brief historical introduction to the non-integer calculus of variations and then we present recent results on the fractional calculus of variations.

For more information about this subject, we refer the reader to the books (Almeida, Pooseh and Torres, 2015; Malinowska, Odzijewicz and Torres, 2015; Malinowska and Torres, 2012; van Brunt, 2004).

\subsection{The classical calculus of variations}

The calculus of variations is a field of mathematical analysis that concerns with finding extrema (maxima or minima) for functionals, i.e., concerns with the problem of finding a function for which the value of a certain integral is either the largest or the smallest possible.

In this context, a functional is a mapping from a set of functions to the real numbers, i.e., it receives a function and produces a real number. Let $D \subseteq C^{2}([a, b] ; \mathbb{R})$ be a linear space endowed with a norm $\|\cdot\|$. The cost functional $\mathcal{J}: D \rightarrow \mathbb{R}$ is generally of the form

$$
\mathcal{J}(x)=\int_{a}^{b} L\left(t, x(t), x^{\prime}(t)\right) d t
$$

where $t \in[a, b]$ is the independent variable, usually called time, and $x(t) \in \mathbb{R}$ is a function. The integrand $L:[a, b] \times \mathbb{R}^{2} \rightarrow \mathbb{R}$, that depends on the function 
$x$, its derivative $x^{\prime}$ and the independent variable $t$, is a real-valued function, called the Lagrangian.

The roots of the calculus of variations appear in works of Greek thinkers, such as Queen Dido or Aristotle in the late of the 1st century BC. During the 17th century, some physicists and mathematicians (Galileo, Fermat, Newton, among others) investigated some variational problems, but in general they did not use variational methods to solve them. The development of the calculus of variations began with a problem posed by Johann Bernoulli in 1696, called the brachistochrone problem: given two points $A$ and $B$ in a vertical plane, what is the curve traced out by a point acted on only by gravity, which starts at $A$ and reaches $B$ in minimal time? The curve that solves the problem is called the brachistochrone. This problem caught the attention of some mathematicians including Jakob Bernoulli, Leibniz, L'Hôpital and Newton, which presented also a solution for the brachistochrone problem. Integer variational calculus is still, nowadays, a revelant area of research. It plays a significant role in many areas of science, physics, engineering, economics, and applied mathematics.

The classical variational problem, considered by Leonhard Euler, is stated as follows.

Let $a, b \in \mathbb{R}$. Among all functions $x \in D$, find the ones that minimize (or maximize) the functional $\mathcal{J}: D \rightarrow \mathbb{R}$, where

$$
\mathcal{J}(x)=\int_{a}^{b} L\left(t, x(t), x^{\prime}(t)\right) d t
$$

subject to the boundary conditions

$$
x(a)=x_{a}, \quad x(b)=x_{b},
$$

with $x_{a}, x_{b}$ fixed reals and the Lagrangian $L$ satisfying some smoothness properties. Usually, we say that a function is "sufficiently smooth" for a particular development if all required actions (integration, differentiation, ...) are possible.

Definition 23 A trajectory $x \in C^{2}([a, b] ; \mathbb{R})$ is said to be an admissible trajectory if it satisfies all the constraints of the problem along the interval $[a, b]$. The set of admissible trajectories is denoted by $D$.

To discuss maxima and minima of functionals, we need to introduce the following definition.

Definition 24 We say that $x^{\star} \in D$ is a local extremizer to the functional $\mathcal{J}: D \rightarrow \mathbb{R}$ if there exists some real $\epsilon>0$, such that

$$
\forall x \in D: \quad\left\|x^{\star}-x\right\|<\epsilon \Rightarrow \mathcal{J}\left(x^{\star}\right)-\mathcal{J}(x) \leq 0 \vee \mathcal{J}\left(x^{\star}\right)-\mathcal{J}(x) \geq 0 .
$$

In this context, as we are dealing with functionals defined on functions, we need to clarify the term of directional derivatives, here called variations. The concept of variation of a functional is central to obtain the solution of variational problems. 
Definition 25 Let $\mathcal{J}$ be defined on $D$. The first variation of a functional $\mathcal{J}$ at $x \in D$ in the direction $h \in D$ is defined by

$$
\left.\delta \mathcal{J}(x, h)=\lim _{\epsilon \rightarrow 0} \frac{\mathcal{J}(x+\epsilon h)-\mathcal{J}(x)}{\epsilon}=\frac{d}{d \epsilon} \mathcal{J}(x+\epsilon h)\right]_{\epsilon=0},
$$

where $x$ and $h$ are functions and $\epsilon$ is a scalar, whenever the limit exists.

Definition 26 A direction $h \in D, h \neq 0$, is said to be an admissible variation for $\mathcal{J}$ at $y \in D$ if

1. $\delta \mathcal{J}(x, h)$ exists;

2. $x+\epsilon h \in D$ for all sufficiently small $\epsilon$.

With the condition that $\mathcal{J}(x)$ be a local extremum and the definition of variation, we have the following result that offers a necessary optimality condition for problems of calculus of variations (van Brunt, 2004).

Theorem 15 Let $\mathcal{J}$ be a functional defined on D. If $x^{\star}$ minimizes (or maximizes) the functional $\mathcal{J}$ over all functions $x:[a, b] \rightarrow \mathbb{R}$ satisfying boundary conditions (2.3), then

$$
\delta \mathcal{J}\left(x^{\star}, h\right)=0
$$

for all admissible variations $h$ at $x^{\star}$.

\subsubsection{Euler-Lagrange equations}

Although the calculus of variations was born with Johann's problem, it was with the work of Euler in 1742 and the one of Lagrange in 1755 that a systematic theory was developed. The common procedure to address such variational problems consists in solving a differential equation, called the Euler-Lagrange equation, which every minimizer/maximizer of the functional must satisfy.

In Lemma 16, we review an important result to transform the necessary condition of extremum in a differential equation, free of integration with an arbitrary function. In literature, it is known as the fundamental lemma of the calculus of variations.

Lemma 16 Let $x$ be continuous in $[a, b]$ an let $h$ be an arbitrary function on $[a, b]$ such that it is continuous and $h(a)=h(b)=0$. If

$$
\int_{a}^{b} x(t) h(t) d t=0
$$

for all such $h$, then $x(t)=0$ for all $t \in[a, b]$.

For the sequel, we denote by $\partial_{i} z, i \in\{1,2, \ldots, M\}$, with $M \in \mathbb{N}$, the partial derivative of a function $z: \mathbb{R}^{M} \rightarrow \mathbb{R}$ with respect to its $i$ th argument. Now we can formulate the necessary optimality condition for the classical variational problem (van Brunt, 2004). 
Theorem 17 If $x$ is an extremizing of the functional (2.2) on D, subject to (2.3), then $x$ satisfies

$$
\partial_{2} L\left(t, x(t), x^{\prime}(t)\right)-\frac{d}{d t} \partial_{3} L\left(t, x(t), x^{\prime}(t)\right)=0
$$

for all $t \in[a, b]$.

To solve this second order differential equation, the two given boundary conditions (2.3) provide sufficient information to determine the two arbitrary constants.

Definition 27 A curve $x$ that is a solution of the Euler-Lagrange differential equation will be called an extremal of $\mathcal{J}$.

\subsubsection{Problems with variable endpoints}

In the basic variational problem considered previously, the functional $\mathcal{J}$ to minimize (or maximize) is subject to given boundary conditions of the form

$$
x(a)=x_{a}, \quad x(b)=x_{b},
$$

where $x_{a}, x_{b} \in \mathbb{R}$ are fixed. It means that the solution of the problem, $x$, needs to pass through the prescribed points. This variational problem is called a fixed endpoints variational problem. The Euler-Lagrange equation (2.4) is normally a second-order differential equation containing two arbitrary constants, so with two given boundary conditions provided, they are sufficient to determine the two constants.

However, in some areas, like physics and geometry, the variational problems do not impose the appropriate number of boundary conditions. In these cases, when one or both boundary conditions are missing, that is, when the set of admissible functions may take any value at one or both of the boundaries, then one or two auxiliary conditions, known as the natural boundary conditions or transversality conditions, need to be obtained in order to solve the equation (van Brunt, 2004):

$$
\left[\frac{\partial L\left(t, x(t), x^{\prime}(t)\right)}{\partial x^{\prime}}\right]_{t=a}=0 \quad \text { and/or } \quad\left[\frac{\partial L\left(t, x(t), x^{\prime}(t)\right)}{\partial x^{\prime}}\right]_{t=b}=0 .
$$

There are different types of variational problems with variable endpoints:

- Free terminal point - one boundary condition at the initial time $(x(a)=$ $\left.x_{a}\right)$. The terminal point is free $(x(b) \in \mathbb{R})$;

- Free initial point - one boundary condition at the final time $\left(x(b)=x_{b}\right)$. The initial point is free $(x(a) \in \mathbb{R})$;

- Free endpoints - both endpoints are free $(x(a) \in \mathbb{R}, x(b) \in \mathbb{R})$;

- Variable endpoints - the initial point $x(a)$ or/and the endpoint $x(b)$ is variable on a certain set, for example, on a prescribed curve. 
Another generalization of the variational problem consists to find an optimal curve $x$ and the optimal final time $T$ of the variational integral, $T \in[a, b]$. This problem is known in the literature as a free-time problem (Chiang, 1992). An example is the following free-time problem with free terminal point. Let $D$ denote the subset $C^{2}([a, b] ; \mathbb{R}) \times[a, b]$ endowed with a norm $\|(\cdot, \cdot)\|$. Find the local minimizers of the functional $\mathcal{J}: D \rightarrow \mathbb{R}$, with

$$
\mathcal{J}(x, T)=\int_{a}^{T} L\left(t, x(t), x^{\prime}(t)\right) d t,
$$

over all $(x, T) \in D$ satisfying the boundary condition $x(a)=x_{a}$, with $x_{a} \in \mathbb{R}$ fixed. The terminal time $T$ and the terminal state $x(T)$ are here both free.

Definition 28 We say that $\left(x^{\star}, T^{\star}\right) \in D$ is a local extremizer (minimizer or maximizer) to the functional $\mathcal{J}: D \rightarrow \mathbb{R}$ as in (2.6) if there exists some $\epsilon>0$ such that, for all $(x, T) \in D$,

$$
\left\|\left(x^{\star}, T^{\star}\right)-(x, T)\right\|<\epsilon \Rightarrow J\left(x^{\star}, T^{\star}\right) \leq J(x, T) \vee J\left(x^{\star}, T^{\star}\right) \geq J(x, T) .
$$

To develop a necessary optimality condition to problem (2.6) for an extremizer $\left(x^{\star}, T^{\star}\right)$, we need to consider an admissible variation of the form:

$$
\left(x^{\star}+\epsilon h, \quad T^{\star}+\epsilon \Delta T\right),
$$

where $h \in C^{1}([a, b] ; \mathbb{R})$ is a perturbing curve that satisfies the condition $h(a)=0, \epsilon$ represents a small real number, and $\Delta T$ represents an arbitrarily chosen small change in $T$. Considering the functional $\mathcal{J}(x, T)$ in this admissible variation, we get a function of $\epsilon$, where the upper limit of integration will also vary with $\epsilon$ :

$$
\mathcal{J}\left(x^{\star}+\epsilon h, \quad T^{\star}+\epsilon \Delta T\right)=\int_{a}^{T^{\star}+\epsilon \Delta T} L\left(t,\left(x^{\star}+\epsilon h\right)(t),\left(x^{\star}+\epsilon h\right)^{\prime}(t)\right) d t .
$$

To find the first-order necessary optimality condition, we need to determine the derivative of (2.7) with respect to $\epsilon$ and set it equal to zero. By doing it, we obtain three terms on the equation, where the Euler-Lagrange equation emerges from the first term, and the other two terms, which depend only on the terminal time $T$, give the transversality conditions.

\subsubsection{Constrained variational problems}

Variational problems are often subject to one or more constraints (holonomic constraints, integral constraints, dynamic constraints, ...). Isoperimetric problems are a special class of constrained variational problems for which the admissible functions are needed to satisfy an integral constraint.

Here we review the classical isoperimetric variational problem. The classical variational problem, already defined, may be modified by demanding that 
the class of potential extremizing functions also satisfy a new condition, called an isoperimetric constraint, of the form

$$
\int_{a}^{b} g\left(t, x(t), x^{\prime}(t)\right) d t=C
$$

where $g$ is a given function of $t, x$ and $x^{\prime}$, and $C$ is a given real number.

The new problem is called an isoperimetric problem and encompasses an important family of variational problems. In this case, the variational problems are often subject to one or more constraints involving an integral of a given function (Fraser, 1992). Some classical examples of isoperimetric problems appear in geometry. The most famous example consists in finding the curve of a given perimeter that bounds the greatest area and the answer is the circle. Isoperimetric problems are an important type of variational problems, with applications in different areas, like geometry, astronomy, physics, algebra or analysis.

In the next theorem, we present a necessary condition for a function to be an extremizer to a classical isoperimetric problem, obtained via the concept of Lagrange multiplier (van Brunt, 2004).

Theorem 18 Consider the problem of minimizing (or maximizing) the functional $\mathcal{J}$, defined by (2.2), on $D$ given by those $x \in C^{2}([a, b] ; \mathbb{R})$ satisfying the boundary conditions (2.3) and an integral constraint of the form

$$
\mathcal{G}=\int_{a}^{b} g\left(t, x(t), x^{\prime}(t)\right) d t=C,
$$

where $g:[a, b] \times \mathbb{R}^{2} \rightarrow \mathbb{R}$ is a twice continuously differentiable function. Suppose that $x$ gives a local minimum (or maximum) to this problem. Assume that $\delta \mathcal{G}(x, h)$ does not vanish for all $h \in D$. Then, there exists a constant $\lambda$ such that $x$ satisfies the Euler-Lagrange equation

$$
\partial_{2} F\left(t, x(t), x^{\prime}(t), \lambda\right)-\frac{d}{d t} \partial_{3} F\left(t, x(t), x^{\prime}(t), \lambda\right)=0,
$$

where $F\left(t, x, x^{\prime}, \lambda\right)=L\left(t, x, x^{\prime}\right)-\lambda g\left(t, x, x^{\prime}\right)$.

Remark 19 The constant $\lambda$ is called a Lagrange multiplier.

Observe that $\delta \mathcal{G}(x, h)$ does not vanish for all $h \in D$ if $x$ does not satisfies the Euler-Lagrange equation with respect to the isoperimetric constraint, that is, $x$ is not an extremal for $\mathcal{G}$.

\subsection{Fractional calculus of variations}

The first connection between fractional calculus and the calculus of variations appeared in the XIX century, with Niels Abel (Abel, 1923). In 1823, Abel 
applied fractional calculus in the solution of an integral equation involved in a generalization of the tautochrone problem. Only in the XX century, however, both areas were joined in an unique research field: the fractional calculus of variations.

The fractional calculus of variations deals with problems in which the functional, the constraint conditions, or both, depend on some fractional operator Almeida. Pooseh and Torres, 2015; Malinowska. Odzijewicz and Torres, 2015; Malinowska and Torres, 2012) and the main goal is to find functions that extremize such a fractional functional. By inserting fractional operators that are non-local in variational problems, they are suitable for developing some models possessing memory effects.

This is a fast growing subject, and different approaches have been developed by considering different types of Lagrangians, e.g., depending on Riemann-Liouville or Caputo fractional derivatives, fractional integrals, and mixed integer-fractional order operators (see, for example, (Almeida and Torres 2009; Askari and Ansari, 2016; Atanacković, Koniik and Pilipović, 2008; Baleanu, 2008; Baleanu et al., 2010; Cresson, 2007; Rabei et al., 2007; Tarasov, 2006)). In recent years, there has been a growing interest in the area of fractional variational calculus and its applications, which include classical and quantum mechanics, field theory and optimal control.

Although the origin of fractional calculus goes back more than three centuries, the calculus of variations with fractional derivatives has born only in 1996-1997 with the works of F. Riewe. In his works, Riewe obtained a version of the Euler-Lagrange equations for problems of the calculus of variations with fractional derivatives, when investigating non-conservative Lagrangian and Hamiltonian mechanics (Riewe, 1996, 1997). Agrawal continued the study of the fractional Euler-Lagrange equations (Agrawal, 2002, 2006, 2007), for some kinds of fractional variational problems, for example, problems with only one dependent variable, for functionals with different orders of fractional derivatives, for several functions, involving both Riemann-Liouville and Caputo derivatives, etc. The most common fractional operators considered in the literature take into account the past of the process, that is, one usually uses left fractional operators. But, in some cases, we may be also interested in the future of the process, and the computation of $\alpha(\cdot)$ to be influenced by it. In that case, right fractional derivatives are then considered.

\subsubsection{Fractional Euler-Lagrange equations}

Similarly to the classical variational calculus, the common procedure to address such fractional variational problems consists in solving a fractional differential equation, called the fractional Euler-Lagrange equation, which every minimizer/maximizer of the functional must satisfy. With the help of the boundary conditions imposed on the problem at the initial time $t=a$ and at the terminal time $t=b$, one solves, often with the help of some numerical procedure, the fractional differential equation and obtain a possible so- 
lution to the problem (Blaszczvk and Ciesielski, 2014; Lotfi and Yousefi, 2013; Pooseh, Almeida and Torres, 2013; Sumelka and Blaszczyk, 2014; Xu and Agrawal, 2014).

Referring again to Riewe's works (Riewe, 1996, 1997), friction forces are described with Lagrangians that contain fractional derivatives. Precisely, for $r, N$ and $N^{\prime}$ natural numbers and assuming $x:[a, b] \rightarrow \mathbb{R}^{r}, \alpha_{i}, \beta_{j} \in[0,1]$ with $i=1, \ldots, N, j=1, \ldots, N^{\prime}$, the functional defined by Riewe is

$\mathcal{J}(x)=\int_{a}^{b} L\left({ }_{a} D_{t}^{\alpha_{1}}[x](t), \ldots,{ }_{a} D_{t}^{\alpha_{N}}[x](t),{ }_{t} D_{b}^{\beta_{1}}[x](t), \ldots,{ }_{t} D_{b}^{\beta_{N^{\prime}}}[x](t), x(t), t\right) d t$.

He proved that any solution $x$ of the variational problem of extremizing the functional (2.10), satisfies the following necessary condition:

Theorem 20 Considering the variational problem of minimizing (or maximizing) the functional (2.10), the fractional Euler-Lagrange equation is

$$
\sum_{i=1}^{N}{ }_{t} D_{b}^{\alpha_{i}}\left[\partial_{i} L\right]+\sum_{i=1}^{N^{\prime}}{ }_{a} D_{t}^{\beta_{i}}\left[\partial_{i+N} L\right]+\partial_{N^{\prime}+N+1} L=0
$$

Riewe also illustrated his results considering the classical problem of linear friction (Riewe, 1996).

In what follows, we are concerned with problems of the fractional calculus of variations where the functional depends on a combined fractional Caputo derivative with constant orders $\alpha$ and $\beta$ (Malinowska and Torres, 2011, Definition 15).

Let $D$ denote the set of all functions $x:[a, b] \rightarrow \mathbb{R}^{N}$, endowed with a norm $\|\cdot\|$ in $\mathbb{R}^{N}$. Consider the following problem: find a function $x \in D$ for which the functional

$$
\mathcal{J}(x)=\int_{a}^{b} L\left(t, x(t),{ }^{C} D_{\gamma}^{\alpha, \beta} x(t)\right) d t
$$

subject to given boundary conditions

$$
x(a)=x_{a}, \quad x(b)=x_{b}
$$

archives a minimum, where $t \in[a, b], x_{a}, x_{b} \in \mathbb{R}^{N}, \gamma \in[0,1]$ and the Lagrangian $L$ satisfies some smoothness properties.

Theorem 21 Let $x=\left(x_{1}, \ldots, x_{N}\right)$ be a local minimizer to the problem with the functional (2.11) subject to two boundary conditions, as defined before. Then, $x$ satisfies the system of $N$ fractional Euler-Lagrange equations

$$
\partial_{i} L\left(t, x(t),{ }^{C} D_{\gamma}^{\alpha, \beta} x(t)\right)+D_{1-\gamma}^{\beta, \alpha} \partial_{N+i} L\left(t, x(t),{ }^{C} D_{\gamma}^{\alpha, \beta} x(t)\right)=0,
$$

$i=2, \ldots, N+1$, for all $t \in[a, b]$. 
For a proof of the last result, see (Malinowska and Torres, 2010). Observe that, if the orders $\alpha$ and $\beta$ go to 1 , and if $\gamma=0$ or $\gamma=1$, we obtain a corresponding result in the classical context of the calculus of variations. In fact, considering $\alpha$ and $\beta$ going to 1 , the fractional derivatives ${ }_{a}^{C} D_{t}^{\alpha}$ and ${ }_{a} D_{t}^{\alpha}$ coincide with the classical derivative $\frac{d}{d t}$; and similarly, ${ }_{t}^{C} D_{b}^{\beta}$ and ${ }_{t} D_{b}^{\beta}$ coincide with the classical derivative $-\frac{d}{d t}$.

Variational problems with free endpoints and transversality conditions are also relevant subjects in the fractional calculus of variations. The subject of free boundary points in fractional variational problems was first considered by Agrawal in (Agrawal, 2006). In that work, he studied the Euler-Lagrange equation and transversality conditions for the case when both initial and final times are given and the admissible functions are specified at the initial time but are unspecified at the final time. After that, some free-time variational problems involving fractional derivatives or/and fractional integrals were studied (Almeida and Malinowska, 2013; Odzijewicz, Malinowska and Torres, 2012a).

Sometimes, the analytic solution of the fractional Euler-Lagrange equation is very difficult to obtain and, in this case, some numerical methods have been developed to solve the variational problem Almeida, Pooseh and Torres, 2015).

\subsubsection{Fractional variational problems of variable-order}

In recent years, motivated by the works of Samko and Ross, where they investigated integrals and derivatives not of a constant but of variable-order (Samko, 1995; Samko and Ross, 1993), some problems of the calculus of variations involving derivatives of variable fractional order have appeared (Atanacković and Pilipović, 2011; Odzijewicz, Malinowska and Torres, 2012b).

Considering Definition 19 of the generalized fractional integral of operator $K_{P}$, (Malinowska, Odzijewicz and Torres, 2015) presented a new variational problem, where the functional was defined by a given kernel. For appropriate choices of the kernel $k$ and the set $P$, we can obtain a variable-order fractional variational problem (see Remark 9).

Let $P=\langle a, t, b, \lambda, \mu\rangle$. Consider the functional $\mathcal{J}$ in $\mathbf{A}\left(x_{a}, x_{b}\right)$ defined by:

$$
\mathcal{J}[x]=\int_{a}^{b} L\left(x(t), K_{P}[x](t), x^{\prime}(t), B_{P}[x](t), t\right) d t,
$$

where $\mathbf{A}\left(x_{a}, x_{b}\right)$ is the set

$$
\left\{x \in C^{1}([a, b] ; \mathbb{R}): x(a)=x_{a}, x(b)=x_{b}, K_{P}[x], B_{P}[x] \in C([a, b] ; \mathbb{R})\right\},
$$

and $K_{P}$ is the generalized fractional integral operator with kernel belonging to $L_{q}(\Delta ; \mathbb{R})$ and $B_{P}$ the generalized fractional derivative of Caputo type.

The optimality condition for the problem that consists to determine a function that minimize (or maximize) the functional (2.13) is given in the following theorem (Malinowska, Odzijewicz and Torres, 2015). 
Theorem 22 Let $x \in \mathbf{A}\left(x_{a}, x_{b}\right)$ be a minimizer of functional (2.13). Then, $x$ satisfies the following Euler-Lagrange equation:

$$
\begin{aligned}
& \frac{d}{d t}\left[\partial_{3} L\left(\star_{x}\right)(t)\right]+A_{P^{*}}\left[\tau \longmapsto \partial_{4} L\left(\star_{x}\right)(\tau)\right](t) \\
& \quad=\partial_{1} L\left(\star_{x}\right)(t)+K_{P^{*}}\left[\tau \longmapsto \partial_{2} L\left(\star_{x}\right)(\tau)\right](t),
\end{aligned}
$$

where $\left(\star_{x}\right)(t)=\left(x(t), K_{P}[x](t), x^{\prime}(t), B_{P}[x](t), t\right)$, for $t \in(a, b)$.

Observe that, if functional (2.13) does not depends on the generalized fractional operators $K_{P}$ and $B_{P}$, this problem coincide with the classical variational problem and Theorem 22 reduces to Theorem 17 .

Let $\Delta:=\left\{(t, \tau) \in \mathbb{R}^{2}: a \leq \tau<t \leq b\right\}$ and let $1<p<\infty$ and $q$ be the adjoint of $p$. A special case of this problem is obtained when we consider $\alpha: \Delta \rightarrow[0,1-\delta]$ with $\delta>1 / p$ and the kernel is defined by

$$
k^{\alpha}(t, \tau)=\frac{1}{\Gamma(1-\alpha(t, \tau))}(t-\tau)^{-\alpha(t, \tau)}
$$

in $L_{q}(\Delta ; \mathbb{R})$. The next results provides necessary conditions of optimality (Malinowska, Odzijewicz and Torres, 2015).

Theorem 23 Consider the problem of minimizing a functional

$$
\mathcal{J}[x]=\int_{a}^{b} L\left(x(t),{ }_{a} I_{t}^{1-\alpha(\cdot, \cdot)}[x](t), x^{\prime}(t),{ }_{a}^{C} D_{t}^{\alpha(\cdot, \cdot)}[x](t), t\right) d t
$$

subject to boundary conditions

$$
x(a)=x_{a}, \quad x(b)=x_{b},
$$

where $x^{\prime},{ }_{a} I_{t}^{1-\alpha(\cdot, \cdot)}[x],{ }_{a}^{C} D_{t}^{\alpha(\cdot, \cdot)}[x] \in C([a, b] ; \mathbb{R})$. Then, if $x \in C^{1}([a, b] ; \mathbb{R})$ minimizes (or maximizes) the functional (2.15) subject to (2.16), then it satisfies the following Euler-Lagrange equation:

$$
\begin{aligned}
\partial_{1} L & \left(x(t),{ }_{a} I_{t}^{1-\alpha(\cdot, \cdot)}[x](t), x^{\prime}(t),{ }_{a}^{C} D_{t}^{\alpha(\cdot, \cdot)}[x](t), t\right) \\
& -\frac{d}{d t} \partial_{3} L\left(x(t),{ }_{a} I_{t}^{1-\alpha(\cdot, \cdot)}[x](t), x^{\prime}(t),{ }_{a}^{C} D_{t}^{\alpha(\cdot, \cdot)}[x](t), t\right) \\
& +{ }_{t} I_{b}^{1-\alpha(\cdot, \cdot)}\left[\partial_{2} L\left(x(\tau),{ }_{a} I_{\tau}^{1-\alpha(\cdot, \cdot)}[x](\tau), x^{\prime}(\tau),{ }_{a}^{C} D_{\tau}^{\alpha(\cdot, \cdot)}[x](\tau), \tau\right)\right](t) \\
& +{ }_{t} D_{b}^{\alpha(\cdot, \cdot)}\left[\partial_{4} L\left(x(\tau),{ }_{a} I_{\tau}^{1-\alpha(\cdot, \cdot)}[x](\tau), x^{\prime}(\tau),{ }_{a}^{C} D_{\tau}^{\alpha(\cdot, \cdot)}[x](\tau), \tau\right)\right](t)=0 .
\end{aligned}
$$

In fact, the use of fractional derivatives of constant order in variational problems may not be the best option, since trajectories are a dynamic process, and the order may vary. Therefore, it is important to consider the order to be a function, $\alpha(\cdot)$, depending on time. Then we may seek what is the best function 
$\alpha(\cdot)$ such that the variable-order fractional differential equation $D^{\alpha(\cdot)} x(t)=$ $f(t, x(t))$ better describes the process under study.

This approach is very recent, and many work has to be done for a complete study of the subject (see, e.g., Atangana and Kilicman, 2014; Coimbra. Soon and Kobavashi, 2005; Samko and Ross, 1993; Sheng et al., 2011; Valério et al., 2009)).

\section{References}

Abel NH (1823) Solution de quelques problèmes à l'aide d'intégrales définies. Mag. Naturv. 1(2):1-127

Agrawal OP (2002) Formulation of Euler-Lagrange equations for fractional variational problems. J. Math. Anal. Appl. 272:368-379

Agrawal OP (2006) Fractional variational calculus and the transversality conditions. J. Phys. A: Math. Gen. 39(33):10375-10384

Agrawal OP (2007) Generalized Euler-Lagrange equations and transversality conditions for FVPs in terms of the Caputo derivative. J. Vib. Control 13(9-10):1217-1237

Almeida R, Malinowska AB (2013) Generalized transversality conditions in fractional calculus of variations. Commun. Nonlinear Sci. Numer. Simul. 18(3):443-452

Almeida R, Pooseh S, Torres DFM (2015) Computational Methods in the Fractional Calculus of Variations. Imperial College Press, London

Almeida R, Torres DFM (2009) Calculus of variations with fractional derivatives and fractional integrals. Appl. Math. Lett. 22(12):1816-1820 arXiv:0907.1024

Askari H, Ansari A (2016) Fractional calculus of variations with a generalized fractional derivative. Fract. Differ. Calc. 6:57-72

Atanacković TM, Konjik S, Pilipović S (2008) Variational problems with fractional derivatives: Euler-Lagrange equations. J. Phys. A 41(9):095201, $12 \mathrm{pp}$

Atanacković TM, Pilipovic S (2011) Hamilton's principle with variable order fractional derivatives. Fract. Calc. Appl. Anal. 14:94-109

Atangana A, Kilicman A (2014) On the generalized mass transport equation to the concept of variable fractional derivative. Math. Probl. Eng. 2014:Art. ID $542809,9 \mathrm{pp}$

Baleanu D (2008) New applications of fractional variational principles. Rep. Math. Phys. 61(2):199-206

Baleanu D, Golmankhaneh AK, Nigmatullin R, Golmankhaneh AK (2010) Fractional Newtonian mechanics. Cent. Eur. J. Phys. 8(1):120-125

Blaszczyk T, Ciesielski M (2014) Numerical solution of fractional SturmLiouville equation in integral form. Fract. Calc. Appl. Anal. 17(2):307-320 Chiang AC (1992) Elements of Dynamic Optimization. McGraw-Hill, Inc., Singapore 
Coimbra CFM, Soon CM, Kobayashi MH (2005) The variable viscoelasticity operator. Annalen der Physik 14(6):378-389

Cresson J (2007) Fractional embedding of differential operators and Lagrangian systems. J. Math. Phys. 48(3):033504, 34 pp

Fraser C (1992) Isoperimetric problems in variatonal calculus of Euler and Lagrange. Historia Mathematica 19:4-23

Lotfi A, Yousefi SA (2013) A numerical technique for solving a class of fractional variational problems. J. Comput. Appl. Math. 237(1):633-643

Malinowska AB, Odzijewicz T, Torres DFM (2015) Advanced Methods in the Fractional Calculus of Variations. Springer Briefs in Applied Sciences and Technology, Springer, Cham

Malinowska AB, Torres DFM (2010) Fractional variational calculus in terms of a combined Caputo derivative. Proceedings of FDA'10, The 4th IFAC Workshop on Fractional Differentiation and its Applications, Badajoz, Spain, October 18-20, 2010 (Eds: I. Podlubny, B. M. Vinagre Jara, YQ. Chen, V. Feliu Batlle, I. Tejado Balsera):Article no. FDA10-084, 6 pp. arXiv: 1007.0743

Malinowska AB, Torres DFM (2011) Fractional calculus of variations for a combined Caputo derivative. Fract. Calc. Appl. Anal. 14(4):523-537 arXiv: 1109.4664

Malinowska AB, Torres DFM (2012) Introduction to the Fractional Calculus of Variations. Imp. Coll. Press, London

Odzijewicz T, Malinowska AB, Torres DFM (2012a) Fractional variational calculus with classical and combined Caputo derivatives. Nonlinear Anal. 75(3):1507-1515 arXiv: 1101.2932

Odzijewicz T, Malinowska AB, Torres DFM (2012b) Variable order fractional variational calculus for double integrals. Proceedings of the 51st IEEE Conference on Decision and Control, December 10-13, 2012, Maui, Hawaii:Art. no. 6426489, 6873-6878. arXiv: 1209.1345

Pooseh S, Almeida R, Torres DFM (2013) Discrete direct methods in the fractional calculus of variations. Comput. Math. Appl. 66(5):668-676 arXiv: 1205.4843

Rabei EM, Nawafleh KI, Hijjawi RS, Muslih SI, Baleanu D (2007) The Hamilton formalism with fractional derivatives. J. Math. Anal. Appl. 327(2):891897

Riewe F (1996) Nonconservative Lagrangian and Hamiltonian mechanics. Phys. Rev. E (3) 53(2):1890-1899

Riewe F (1997) Mechanics with fractional derivatives. Phys. Rev. E (3) $55(3): 3581-3592$

Samko SG (1995) Fractional integration and differentiation of variable order. Anal. Math. 21(3):213-236

Samko SG, Ross B (1993) Integration and differentiation to a variable fractional order. Integral Transform. Spec. Funct. 1(4):277-300

Sheng H, Sun HG, Coopmans C, Chen YQ, Bohannan GW (2011) A physical experimental study of variable-order fractional integrator and differentiator. Eur. Phys. J. 193(1):93-104 
Sumelka W, Blaszczyk T (2014) Fractional continua for linear elasticity. Arch. Mech. 66(3):147-172

Tarasov VE (2006) Fractional variations for dynamical systems: Hamilton and Lagrange approaches. J. Phys. A 39(26):8409-8425

Valério D, Vinagre G, Domingues J, Costa JS (2009) Variable-order fractional derivatives and their numerical approximations I - real orders. Symposium on Fractional Signals and Systems Lisbon 09, M. Ortigueira et al. (eds.) Lisbon, Portugal

van Brunt B (2004) The Calculus of Variations. Universitext, Springer, New York

Xu Y, Agrawal OP (2014) Models and numerical solutions of generalized oscillator equations. J. Vib. Acoust. 136(5):050903, 7 pp 



\section{Expansion formulas for fractional derivatives}

In this chapter, we present a new numerical tool to solve differential equations involving three types of Caputo derivatives of fractional variable-order. For each one of them, an approximation formula is obtained, which is written in terms of standard (integer order) derivatives only. Estimations for the error of the approximations are also provided. Then, we compare the numerical approximation of some test function with its exact fractional derivative. We present an exemplification of how the presented methods can be used to solve partial fractional differential equations of variable-order.

Let us briefly describe the main contents of the chapter. We begin this chapter by formulating the needed definitions (Section 3.1). Namely, we present three types of Caputo derivatives of variable fractional order. First, we consider one independent variable only; then we generalize for several independent variables. The following Section 3.2 is the main core of the chapter, where we prove approximation formulas for the given fractional operators of variable-order and respectively upper bound formulas for the errors. To test the efficiency of the proposed method, in Section 3.3 we compare the exact fractional derivative of some test function with the numerical approximations obtained from the decomposition formulas given in Section 3.2. To end, in Section 3.4 we apply our method to approximate two physical problems involving Caputo fractional operators of variable-order (a time-fractional diffusion equation and a fractional Burgers' partial differential equation in fluid mechanics) by classical problems that may be solved by well-known standard techniques. 2016).

The results of this chapter first appeared in (Tavares. Almeida and Torres,

\subsection{Caputo-type fractional operators of variable-order}

In the literature of fractional calculus, several different definitions of derivatives are found (Samko. Kilbas and Marichev, 1993). One of those, introduced by (Caputo, 1967) and studied independently by other authors, like 
(Džrbašian and Nersesian, 1968) and (Rabotnov, 1969), has found many applications and seems to be more suitable to model physical phenomena (Dalir and Bashour, 2010; Diethelm, 2004; Machado et al., 2010; Murio and Meiía, 2008; Singh, Saxena and Kumar, 2013; Sweilam and AL-Mrawm, 2011; Yajima and Yamasaki, 2012).

\subsubsection{Caputo derivatives for functions of one variable}

Our goal is to consider fractional derivatives of variable-order, with $\alpha$ depending on time. In fact, some phenomena in physics are better described when the order of the fractional operator is not constant, for example, in the diffusion process in an inhomogeneous or heterogeneous medium, or processes where the changes in the environment modify the dynamic of the particle (Chechkin. Gorenflo and Sokolov, 2005; Santamaria et al., 2006; Sun, Chen and Chen, 2009). Motivated by the above considerations, we introduce three types of Caputo fractional derivatives. The order of the derivative is considered as a function $\alpha(t)$ taking values on the open interval $(0,1)$. To start, we define two different kinds of Riemann-Liouville fractional derivatives.

Definition 29 Given a function $x:[a, b] \rightarrow \mathbb{R}$,

1. the type I left Riemann-Liouville fractional derivative of order $\alpha(t)$ is defined by

$$
{ }_{a} D_{t}^{\alpha(t)} x(t)=\frac{1}{\Gamma(1-\alpha(t))} \frac{d}{d t} \int_{a}^{t}(t-\tau)^{-\alpha(t)} x(\tau) d \tau ;
$$

2. the type I right Riemann-Liouville fractional derivative of order $\alpha(t)$ is defined by

$$
{ }_{t} D_{b}^{\alpha(t)} x(t)=\frac{-1}{\Gamma(1-\alpha(t))} \frac{d}{d t} \int_{t}^{b}(\tau-t)^{-\alpha(t)} x(\tau) d \tau ;
$$

3. the type II left Riemann-Liouville fractional derivative of order $\alpha(t)$ is defined by

$$
{ }_{a} \mathcal{D}_{t}^{\alpha(t)} x(t)=\frac{d}{d t}\left(\frac{1}{\Gamma(1-\alpha(t))} \int_{a}^{t}(t-\tau)^{-\alpha(t)} x(\tau) d \tau\right) ;
$$

4. the type II right Riemann-Liouville fractional derivative of order $\alpha(t)$ is defined by

$$
{ }_{t} \mathcal{D}_{b}^{\alpha(t)} x(t)=\frac{d}{d t}\left(\frac{-1}{\Gamma(1-\alpha(t))} \int_{t}^{b}(\tau-t)^{-\alpha(t)} x(\tau) d \tau\right) .
$$

The Caputo derivatives are given using the previous Riemann-Liouville fractional derivatives. 
Definition 30 Given a function $x:[a, b] \rightarrow \mathbb{R}$,

1. the type I left Caputo derivative of order $\alpha(t)$ is defined by

$$
\begin{aligned}
{ }_{a}^{C} D_{t}^{\alpha(t)} x(t) & ={ }_{a} D_{t}^{\alpha(t)}(x(t)-x(a)) \\
& =\frac{1}{\Gamma(1-\alpha(t))} \frac{d}{d t} \int_{a}^{t}(t-\tau)^{-\alpha(t)}[x(\tau)-x(a)] d \tau ;
\end{aligned}
$$

2. the type I right Caputo derivative of order $\alpha(t)$ is defined by

$$
\begin{aligned}
{ }_{t}^{C} D_{b}^{\alpha(t)} x(t) & ={ }_{t} D_{b}^{\alpha(t)}(x(t)-x(b)) \\
& =\frac{-1}{\Gamma(1-\alpha(t))} \frac{d}{d t} \int_{t}^{b}(\tau-t)^{-\alpha(t)}[x(\tau)-x(b)] d \tau ;
\end{aligned}
$$

3. the type II left Caputo derivative of order $\alpha(t)$ is defined by

$$
\begin{aligned}
{ }_{a}^{C} \mathcal{D}_{t}^{\alpha(t)} x(t) & ={ }_{a} \mathcal{D}_{t}^{\alpha(t)}(x(t)-x(a)) \\
& =\frac{d}{d t}\left(\frac{1}{\Gamma(1-\alpha(t))} \int_{a}^{t}(t-\tau)^{-\alpha(t)}[x(\tau)-x(a)] d \tau\right) ;
\end{aligned}
$$

4. the type II right Caputo derivative of order $\alpha(t)$ is defined by

$$
\begin{aligned}
{ }_{t}^{C} \mathcal{D}_{b}^{\alpha(t)} x(t) & ={ }_{t} \mathcal{D}_{b}^{\alpha(t)}(x(t)-x(b)) \\
& =\frac{d}{d t}\left(\frac{-1}{\Gamma(1-\alpha(t))} \int_{t}^{b}(\tau-t)^{-\alpha(t)}[x(\tau)-x(b)] d \tau\right) ;
\end{aligned}
$$

5. the type III left Caputo derivative of order $\alpha(t)$ is defined by

$$
{ }_{a}^{C} \mathbb{D}_{t}^{\alpha(t)} x(t)=\frac{1}{\Gamma(1-\alpha(t))} \int_{a}^{t}(t-\tau)^{-\alpha(t)} x^{\prime}(\tau) d \tau ;
$$

6. the type III right Caputo derivative of order $\alpha(t)$ is defined by

$$
{ }_{t}^{C} \mathbb{D}_{b}^{\alpha(t)} x(t)=\frac{-1}{\Gamma(1-\alpha(t))} \int_{t}^{b}(\tau-t)^{-\alpha(t)} x^{\prime}(\tau) d \tau .
$$

In contrast with the case when $\alpha$ is a constant, definitions of different types do not coincide.

Theorem 24 The following relations between the left fractional operators hold:

$$
\begin{aligned}
{ }_{a}^{C} D_{t}^{\alpha(t)} x(t)={ }_{a}^{C} \mathbb{D}_{t}^{\alpha(t)} & x(t)+\frac{\alpha^{\prime}(t)}{\Gamma(2-\alpha(t))} \\
& \times \int_{a}^{t}(t-\tau)^{1-\alpha(t)} x^{\prime}(\tau)\left[\frac{1}{1-\alpha(t)}-\ln (t-\tau)\right] d \tau
\end{aligned}
$$


and

$$
\begin{aligned}
&{ }_{a}^{C} D_{t}^{\alpha(t)} x(t)={ }_{a}^{C} \mathcal{D}_{t}^{\alpha(t)} x(t)-\frac{\alpha^{\prime}(t) \Psi(1-\alpha(t))}{\Gamma(1-\alpha(t))} \\
& \quad \times \int_{a}^{t}(t-\tau)^{-\alpha(t)}[x(\tau)-x(a)] d \tau .
\end{aligned}
$$

Proof. Integrating by parts, one gets

$$
\begin{aligned}
{ }_{a}^{C} D_{t}^{\alpha(t)} x(t) & =\frac{1}{\Gamma(1-\alpha(t))} \frac{d}{d t} \int_{a}^{t}(t-\tau)^{-\alpha(t)}[x(\tau)-x(a)] d \tau \\
& =\frac{1}{\Gamma(1-\alpha(t))} \frac{d}{d t}\left[\frac{1}{1-\alpha(t)} \int_{a}^{t}(t-\tau)^{1-\alpha(t)} x^{\prime}(\tau) d \tau\right] .
\end{aligned}
$$

Differentiating the integral, it follows that

$$
\begin{aligned}
& { }_{a}^{C} D_{t}^{\alpha(t)} x(t)=\frac{1}{\Gamma(1-\alpha(t))}\left[\frac{\alpha^{\prime}(t)}{(1-\alpha(t))^{2}} \int_{a}^{t}(t-\tau)^{1-\alpha(t)} x^{\prime}(\tau) d \tau\right. \\
& \left.\quad+\frac{1}{1-\alpha(t)} \int_{a}^{t}(t-\tau)^{1-\alpha(t)} x^{\prime}(\tau)\left[-\alpha^{\prime}(t) \ln (t-\tau)+\frac{1-\alpha(t)}{t-\tau}\right] d \tau\right] \\
& ={ }_{a}^{C} \mathbb{D}_{t}^{\alpha(t)} x(t)+\frac{\alpha^{\prime}(t)}{\Gamma(2-\alpha(t))} \int_{a}^{t}(t-\tau)^{1-\alpha(t)} x^{\prime}(\tau)\left[\frac{1}{1-\alpha(t)}-\ln (t-\tau)\right] d \tau .
\end{aligned}
$$

The second formula follows from direct calculations.

Therefore, when the order $\alpha(t) \equiv c$ is a constant, or for constant functions $x(t) \equiv k$, we have

$$
{ }_{a}^{C} D_{t}^{\alpha(t)} x(t)={ }_{a}^{C} \mathcal{D}_{t}^{\alpha(t)} x(t)={ }_{a}^{C} \mathbb{D}_{t}^{\alpha(t)} x(t) .
$$

Similarly, we obtain the next result.

Theorem 25 The following relations between the right fractional operators hold:

$$
\begin{aligned}
{ }_{t}^{C} D_{b}^{\alpha(t)} x(t)={ }_{t}^{C} \mathbb{D}_{b}^{\alpha(t)} x(t) & +\frac{\alpha^{\prime}(t)}{\Gamma(2-\alpha(t))} \\
& \times \int_{t}^{b}(\tau-t)^{1-\alpha(t)} x^{\prime}(\tau)\left[\frac{1}{1-\alpha(t)}-\ln (\tau-t)\right] d \tau
\end{aligned}
$$

and

$$
{ }_{t}^{C} D_{b}^{\alpha(t)} x(t)={ }_{t}^{C} \mathcal{D}_{b}^{\alpha(t)} x(t)+\frac{\alpha^{\prime}(t) \Psi(1-\alpha(t))}{\Gamma(1-\alpha(t))} \int_{t}^{b}(\tau-t)^{-\alpha(t)}[x(\tau)-x(b)] d \tau .
$$


Theorem 26 Let $x \in C^{1}([a, b], \mathbb{R})$. At $t=a$

$$
{ }_{a}^{C} D_{t}^{\alpha(t)} x(t)={ }_{a}^{C} \mathcal{D}_{t}^{\alpha(t)} x(t)={ }_{a}^{C} \mathbb{D}_{t}^{\alpha(t)} x(t)=0 ;
$$

at $t=b$

$$
{ }_{t}^{C} D_{b}^{\alpha(t)} x(t)={ }_{t}^{C} \mathcal{D}_{b}^{\alpha(t)} x(t)={ }_{t}^{C} \mathbb{D}_{b}^{\alpha(t)} x(t)=0 .
$$

Proof. We start proving the third equality at the initial time $t=a$. We simply note that

$$
\left|{ }_{a}^{C} \mathbb{D}_{t}^{\alpha(t)} x(t)\right| \leq \frac{\left\|x^{\prime}\right\|}{\Gamma(1-\alpha(t))} \int_{a}^{t}(t-\tau)^{-\alpha(t)} d \tau=\frac{\left\|x^{\prime}\right\|}{\Gamma(2-\alpha(t))}(t-a)^{1-\alpha(t)},
$$

which is zero at $t=a$. For the first equality at $t=a$, using equation (3.1), and the two next relations

$$
\left|\int_{a}^{t}(t-\tau)^{1-\alpha(t)} \frac{x^{\prime}(\tau)}{1-\alpha(t)} d \tau\right| \leq \frac{\left\|x^{\prime}\right\|}{(1-\alpha(t))(2-\alpha(t))}(t-a)^{2-\alpha(t)}
$$

and

$$
\begin{aligned}
& \left|\int_{a}^{t}(t-\tau)^{1-\alpha(t)} x^{\prime}(\tau) \ln (t-\tau) d \tau\right| \\
& \leq \frac{\left\|x^{\prime}\right\|}{2-\alpha(t)}(t-a)^{2-\alpha(t)}\left|\ln (t-a)-\frac{1}{2-\alpha(t)}\right|,
\end{aligned}
$$

this latter inequality obtained from integration by parts, we prove that ${ }_{a}^{C} D_{t}^{\alpha(t)} x(t)=0$ at $t=a$. Finally, we prove the second equality at $t=a$ by considering equation (3.2): performing an integration by parts, we get

$$
\left|\int_{a}^{t}(t-\tau)^{-\alpha(t)}[x(\tau)-x(a)] d \tau\right| \leq \frac{\left\|x^{\prime}\right\|}{(1-\alpha(t))(2-\alpha(t))}(t-a)^{2-\alpha(t)}
$$

and so ${ }_{a}^{C} \mathcal{D}_{t}^{\alpha(t)} x(t)=0$ at $t=a$. The proof that the right fractional operators also vanish at the end point $t=b$ follows by similar arguments.

With some computations, a relationship between the Riemann-Liouville and the Caputo fractional derivatives is easily deduced:

$$
\begin{aligned}
{ }_{a} D_{t}^{\alpha(t)} x(t)= & { }_{a}^{C} D_{t}^{\alpha(t)} x(t)+\frac{x(a)}{\Gamma(1-\alpha(t))} \frac{d}{d t} \int_{a}^{t}(t-\tau)^{-\alpha(t)} d \tau \\
={ }_{a}^{C} D_{t}^{\alpha(t)} x(t)+\frac{x(a)}{\Gamma(1-\alpha(t))}(t-a)^{-\alpha(t)} & \\
& \quad+\frac{x(a) \alpha^{\prime}(t)}{\Gamma(2-\alpha(t))}(t-a)^{1-\alpha(t)}\left[\frac{1}{1-\alpha(t)}-\ln (t-a)\right]
\end{aligned}
$$

and 


$$
\begin{aligned}
&{ }_{a} \mathcal{D}_{t}^{\alpha(t)} x(t)={ }_{a}^{C} \mathcal{D}_{t}^{\alpha(t)} x(t)+x(a) \frac{d}{d t}\left(\frac{1}{\Gamma(1-\alpha(t))} \int_{a}^{t}(t-\tau)^{-\alpha(t)} d \tau\right) \\
&={ }_{a}^{C} \mathcal{D}_{t}^{\alpha(t)} x(t)+\frac{x(a)}{\Gamma(1-\alpha(t))}(t-a)^{-\alpha(t)} \\
& \\
& \quad+\frac{x(a) \alpha^{\prime}(t)}{\Gamma(2-\alpha(t))}(t-a)^{1-\alpha(t)}[\Psi(2-\alpha(t))-\ln (t-a)] .
\end{aligned}
$$

For the right fractional operators, we have

$$
\begin{aligned}
{ }_{t} D_{b}^{\alpha(t)} x(t)={ }_{t}^{C} D_{b}^{\alpha(t)} x(t) & +\frac{x(b)}{\Gamma(1-\alpha(t))}(b-t)^{-\alpha(t)} \\
& -\frac{x(b) \alpha^{\prime}(t)}{\Gamma(2-\alpha(t))}(b-t)^{1-\alpha(t)}\left[\frac{1}{1-\alpha(t)}-\ln (b-t)\right]
\end{aligned}
$$

and

$$
\begin{aligned}
{ }_{t} \mathcal{D}_{b}^{\alpha(t)} x(t)={ }_{t}^{C} \mathcal{D}_{b}^{\alpha(t)} & x(t) \\
& +\frac{x(b)}{\Gamma(1-\alpha(t))}(b-t)^{-\alpha(t)} \\
& -\frac{x(b) \alpha^{\prime}(t)}{\Gamma(2-\alpha(t))}(b-t)^{1-\alpha(t)}[\Psi(2-\alpha(t))-\ln (b-t)] .
\end{aligned}
$$

Thus, it is immediate to conclude that if $x(a)=0$, then

$$
{ }_{a} D_{t}^{\alpha(t)} x(t)={ }_{a}^{C} D_{t}^{\alpha(t)} x(t) \text { and } \quad{ }_{a} \mathcal{D}_{t}^{\alpha(t)} x(t)={ }_{a}^{C} \mathcal{D}_{t}^{\alpha(t)} x(t)
$$

and if $x(b)=0$, then

$$
{ }_{t} D_{b}^{\alpha(t)} x(t)={ }_{t}^{C} D_{b}^{\alpha(t)} x(t) \text { and }{ }_{t} \mathcal{D}_{b}^{\alpha(t)} x(t)={ }_{t}^{C} \mathcal{D}_{b}^{\alpha(t)} x(t) .
$$

Next we obtain formulas for the Caputo fractional derivatives of a power function.

Lemma 27 Let $x(t)=(t-a)^{\gamma}$ with $\gamma>0$. Then,

$$
\begin{aligned}
{ }_{a}^{C} D_{t}^{\alpha(t)} x(t)= & \frac{\Gamma(\gamma+1)}{\Gamma(\gamma-\alpha(t)+1)}(t-a)^{\gamma-\alpha(t)} \\
& -\alpha^{\prime}(t) \frac{\Gamma(\gamma+1)}{\Gamma(\gamma-\alpha(t)+2)}(t-a)^{\gamma-\alpha(t)+1} \\
& \times[\ln (t-a)-\Psi(\gamma-\alpha(t)+2)+\Psi(1-\alpha(t))], \\
{ }_{a}^{C} \mathcal{D}_{t}^{\alpha(t)} x(t)= & \frac{\Gamma(\gamma+1)}{\Gamma(\gamma-\alpha(t)+1)}(t-a)^{\gamma-\alpha(t)} \\
& -\alpha^{\prime}(t) \frac{\Gamma(\gamma+1)}{\Gamma(\gamma-\alpha(t)+2)}(t-a)^{\gamma-\alpha(t)+1} \\
& \times[\ln (t-a)-\Psi(\gamma-\alpha(t)+2)], \\
{ }_{a}^{C} \mathbb{D}_{t}^{\alpha(t)} x(t)= & \frac{\Gamma(\gamma+1)}{\Gamma(\gamma-\alpha(t)+1)}(t-a)^{\gamma-\alpha(t)} .
\end{aligned}
$$


Proof. The formula for ${ }_{a}^{C} D_{t}^{\alpha(t)} x(t)$ follows immediately from (Samko and Ross, 1993). For the second equality, one has

$$
\begin{aligned}
{ }_{a}^{C} \mathcal{D}_{t}^{\alpha(t)} x(t) & =\frac{d}{d t}\left(\frac{1}{\Gamma(1-\alpha(t))} \int_{a}^{t}(t-\tau)^{-\alpha(t)}(\tau-a)^{\gamma} d \tau\right) \\
& =\frac{d}{d t}\left(\frac{1}{\Gamma(1-\alpha(t))} \int_{a}^{t}(t-a)^{-\alpha(t)}\left(1-\frac{\tau-a}{t-a}\right)^{-\alpha(t)}(\tau-a)^{\gamma} d \tau\right) .
\end{aligned}
$$

With the change of variables $\tau-a=s(t-a)$, and with the help of the Beta function $B(\cdot, \cdot)$ (see Definition 3 ), we prove that

$$
\begin{aligned}
{ }_{a}^{C} \mathcal{D}_{t}^{\alpha(t)} x(t) & =\frac{d}{d t}\left(\frac{(t-a)^{-\alpha(t)}}{\Gamma(1-\alpha(t))} \int_{0}^{1}(1-s)^{-\alpha(t)} s^{\gamma}(t-a)^{\gamma+1} d s\right) \\
& =\frac{d}{d t}\left(\frac{(t-a)^{\gamma-\alpha(t)+1}}{\Gamma(1-\alpha(t))} B(\gamma+1,1-\alpha(t))\right) \\
& =\frac{d}{d t}\left(\frac{\Gamma(\gamma+1)}{\Gamma(\gamma-\alpha(t)+2)}(t-a)^{\gamma-\alpha(t)+1}\right) .
\end{aligned}
$$

We obtain the desired formula by differentiating this latter expression. The last equality follows in a similar way.

Analogous relations to those of Lemma 27, for the right Caputo fractional derivatives of variable-order, are easily obtained.

Lemma 28 Let $x(t)=(b-t)^{\gamma}$ with $\gamma>0$. Then,

$$
\begin{aligned}
{ }_{t}^{C} D_{b}^{\alpha(t)} x(t)= & \frac{\Gamma(\gamma+1)}{\Gamma(\gamma-\alpha(t)+1)}(b-t)^{\gamma-\alpha(t)} \\
& +\alpha^{\prime}(t) \frac{\Gamma(\gamma+1)}{\Gamma(\gamma-\alpha(t)+2)}(b-t)^{\gamma-\alpha(t)+1} \\
& \times[\ln (b-t)-\Psi(\gamma-\alpha(t)+2)+\Psi(1-\alpha(t))], \\
{ }_{t}^{C} \mathcal{D}_{b}^{\alpha(t)} x(t)= & \frac{\Gamma(\gamma+1)}{\Gamma(\gamma-\alpha(t)+1)}(b-t)^{\gamma-\alpha(t)} \\
& +\alpha^{\prime}(t) \frac{\Gamma(\gamma+1)}{\Gamma(\gamma-\alpha(t)+2)}(b-t)^{\gamma-\alpha(t)+1} \\
& \times[\ln (b-t)-\Psi(\gamma-\alpha(t)+2)], \\
{ }_{t}^{C} \mathbb{D}_{b}^{\alpha(t)} x(t)= & \frac{\Gamma(\gamma+1)}{\Gamma(\gamma-\alpha(t)+1)}(b-t)^{\gamma-\alpha(t)} .
\end{aligned}
$$

With Lemma 27] in mind, we immediately see that

$$
{ }_{a}^{C} D_{t}^{\alpha(t)} x(t) \neq{ }_{a}^{C} \mathcal{D}_{t}^{\alpha(t)} x(t) \neq{ }_{a}^{C} \mathbb{D}_{t}^{\alpha(t)} x(t) .
$$

Also, at least for the power function, it suggests that ${ }_{a}^{C} \mathbb{D}_{t}^{\alpha(t)} x(t)$ may be a more suitable inverse operation of the fractional integral when the order is 
variable. For example, consider functions $x(t)=t^{2}$ and $y(t)=(1-t)^{2}$, and the fractional order $\alpha(t)=\frac{5 t+1}{10}, t \in[0,1]$. Then, $0.1 \leq \alpha(t) \leq 0.6$ for all $t$. Next we compare the fractional derivatives of $x$ and $y$ of order $\alpha(t)$ with the fractional derivatives of constant order $\alpha=0.1$ and $\alpha=0.6$. By Lemma 27, we know that the left Caputo fractional derivatives of order $\alpha(t)$ of $x$ are given by

$$
\begin{aligned}
{ }_{0}^{C} D_{t}^{\alpha(t)} x(t)= & \frac{2}{\Gamma(3-\alpha(t))} t^{2-\alpha(t)} \\
& -\frac{t^{3-\alpha(t)}}{\Gamma(4-\alpha(t))}[\ln (t)-\Psi(4-\alpha(t))+\Psi(1-\alpha(t))], \\
{ }_{0}^{C} \mathcal{D}_{t}^{\alpha(t)} x(t)= & \frac{2}{\Gamma(3-\alpha(t))} t^{2-\alpha(t)}-\frac{t^{3-\alpha(t)}}{\Gamma(4-\alpha(t))}[\ln (t)-\Psi(4-\alpha(t))], \\
{ }_{0}^{C} \mathbb{D}_{t}^{\alpha(t)} x(t)= & \frac{2}{\Gamma(3-\alpha(t))} t^{2-\alpha(t)},
\end{aligned}
$$

while by Lemma 28, the right Caputo fractional derivatives of order $\alpha(t)$ of $y$ are given by

$$
\begin{aligned}
{ }_{t}^{C} D_{1}^{\alpha(t)} y(t)= & \frac{2(1-t)^{2-\alpha(t)}}{\Gamma(3-\alpha(t))} \\
& +\frac{(1-t)^{3-\alpha(t)}}{\Gamma(4-\alpha(t))}[\ln (1-t)-\Psi(4-\alpha(t))+\Psi(1-\alpha(t))], \\
{ }_{t}^{C} \mathcal{D}_{1}^{\alpha(t)} y(t)= & \frac{2(1-t)^{2-\alpha(t)}}{\Gamma(3-\alpha(t))}+\frac{(1-t)^{3-\alpha(t)}}{\Gamma(4-\alpha(t))}[\ln (1-t)-\Psi(4-\alpha(t))], \\
{ }_{t}^{C} \mathbb{D}_{1}^{\alpha(t)} y(t)= & \frac{2(1-t)^{2-\alpha(t)}}{\Gamma(3-\alpha(t))} .
\end{aligned}
$$

For a constant order $\alpha$, we have

$$
{ }_{0}^{C} D_{t}^{\alpha} x(t)=\frac{2}{\Gamma(3-\alpha)} t^{2-\alpha} \quad \text { and } \quad{ }_{t}^{C} D_{1}^{\alpha} y(t)=\frac{2}{\Gamma(3-\alpha)}(1-t)^{2-\alpha} .
$$

The results can be seen in Figure 3.1

\subsubsection{Caputo derivatives for functions of several variables}

Partial fractional derivatives are a natural extension and are defined in a similar way. Let $m \in \mathbb{N}, k \in\{1, \ldots, m\}$, and consider a function $x: \prod_{i=1}^{m}\left[a_{i}, b_{i}\right] \rightarrow \mathbb{R}$ with $m$ variables. For simplicity, we define the vectors

$$
[\tau]_{k}=\left(t_{1}, \ldots, t_{k-1}, \tau, t_{k+1}, \ldots, t_{m}\right) \in \mathbb{R}^{m}
$$

and

$$
(\bar{t})=\left(t_{1}, \ldots, t_{m}\right) \in \mathbb{R}^{m} .
$$




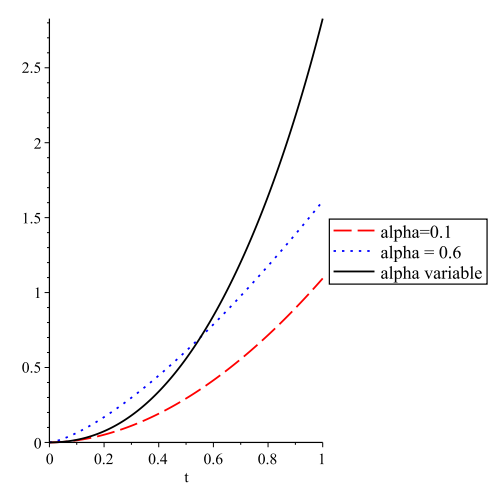

(a) ${ }_{0}^{C} D_{t}^{\alpha(t)} x(t)$

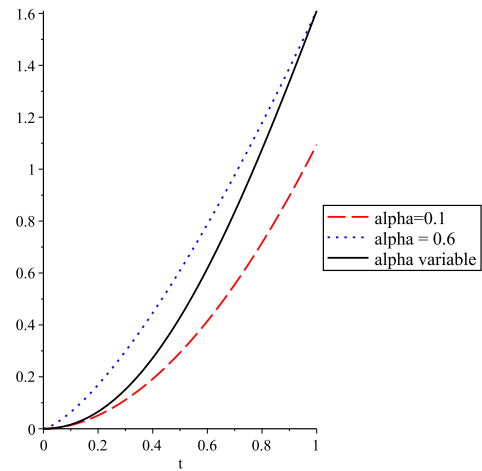

(c) ${ }_{0}^{C} \mathbb{D}_{t}^{\alpha(t)} x(t)$

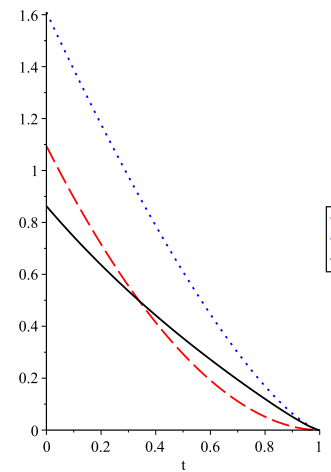

(e) ${ }_{t}^{C} \mathcal{D}_{1}^{\alpha(t)} y(t)$

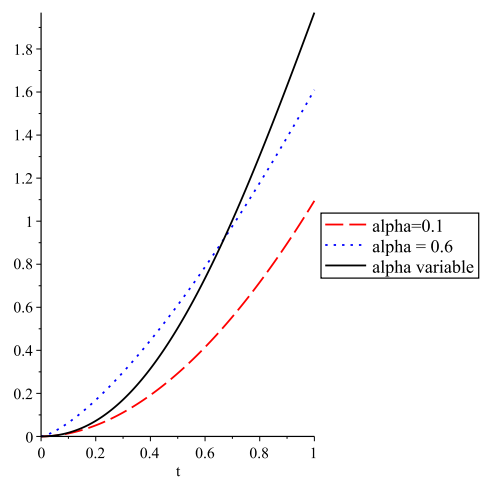

(b) ${ }_{0}^{C} \mathcal{D}_{t}^{\alpha(t)} x(t)$

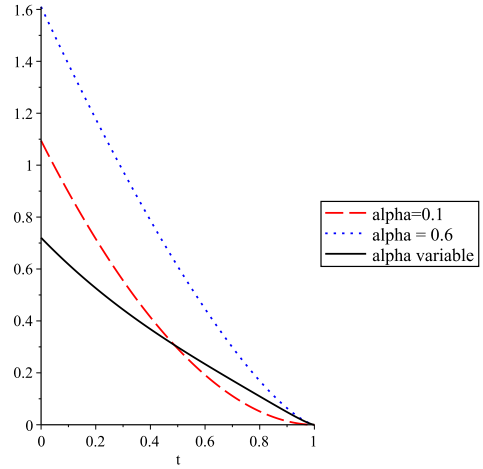

(d) ${ }_{t}^{C} D_{1}^{\alpha(t)} y(t)$

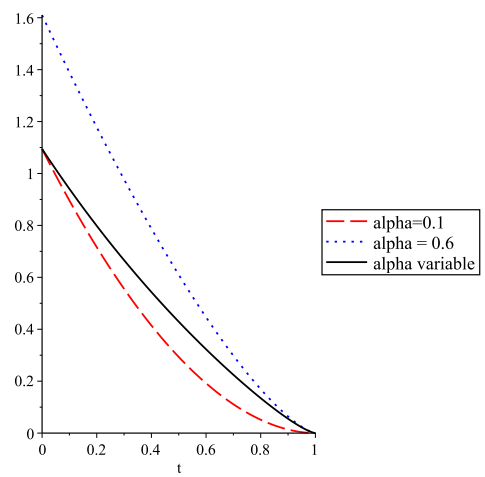

(f) ${ }_{t}^{C} \mathbb{D}_{1}^{\alpha(t)} y(t)$

Fig. 3.1. Comparison between variable-order and constant-order fractional derivatives. 
Definition 31 Given a function $x: \prod_{i=1}^{m}\left[a_{i}, b_{i}\right] \rightarrow \mathbb{R}$ and fractional orders $\alpha_{k}:\left[a_{k}, b_{k}\right] \rightarrow(0,1), k \in\{1, \ldots, m\}$,

1. the type I partial left Caputo derivative of order $\alpha_{k}\left(t_{k}\right)$ is defined by

$$
{ }_{a_{k}}^{C} D_{t_{k}}^{\alpha_{k}\left(t_{k}\right)} x(\bar{t})=\frac{1}{\Gamma\left(1-\alpha_{k}\left(t_{k}\right)\right)} \frac{\partial}{\partial t_{k}} \int_{a_{k}}^{t_{k}}\left(t_{k}-\tau\right)^{-\alpha_{k}\left(t_{k}\right)}\left(x[\tau]_{k}-x\left[a_{k}\right]_{k}\right) d \tau ;
$$

2. the type I partial right Caputo derivative of order $\alpha_{k}\left(t_{k}\right)$ is defined by

$$
{ }_{t_{k}}^{C} D_{b_{k}}^{\alpha_{k}\left(t_{k}\right)} x(\bar{t})=\frac{-1}{\Gamma\left(1-\alpha_{k}\left(t_{k}\right)\right)} \frac{\partial}{\partial t_{k}} \int_{t_{k}}^{b_{k}}\left(\tau-t_{k}\right)^{-\alpha_{k}\left(t_{k}\right)}\left(x[\tau]_{k}-x\left[b_{k}\right]_{k}\right) d \tau ;
$$

3. the type II partial left Caputo derivative of order $\alpha_{k}\left(t_{k}\right)$ is defined by

$$
\begin{aligned}
{ }_{a_{k}}^{C} \mathcal{D}_{t_{k}}^{\alpha_{k}\left(t_{k}\right)} & x(\bar{t}) \\
& =\frac{\partial}{\partial t_{k}}\left(\frac{1}{\Gamma\left(1-\alpha_{k}\left(t_{k}\right)\right)} \int_{a_{k}}^{t_{k}}\left(t_{k}-\tau\right)^{-\alpha_{k}\left(t_{k}\right)}\left(x[\tau]_{k}-x\left[a_{k}\right]_{k}\right) d \tau\right) ;
\end{aligned}
$$

4. the type II partial right Caputo derivative of order $\alpha_{k}\left(t_{k}\right)$ is defined by

$$
\begin{aligned}
{ }_{t_{k}}^{C} \mathcal{D}_{b_{k}}^{\alpha_{k}\left(t_{k}\right)} x(\bar{t}) & \\
& =\frac{\partial}{\partial t_{k}}\left(\frac{-1}{\Gamma\left(1-\alpha_{k}\left(t_{k}\right)\right)} \int_{t_{k}}^{b_{k}}\left(\tau-t_{k}\right)^{-\alpha_{k}\left(t_{k}\right)}\left(x[\tau]_{k}-x\left[b_{k}\right]_{k}\right) d \tau\right) ;
\end{aligned}
$$

5. the type III partial left Caputo derivative of order $\alpha_{k}\left(t_{k}\right)$ is defined by

$$
{ }_{a_{k}}^{C} \mathbb{D}_{t_{k}}^{\alpha_{k}\left(t_{k}\right)} x(\bar{t})=\frac{1}{\Gamma\left(1-\alpha_{k}\left(t_{k}\right)\right)} \int_{a_{k}}^{t_{k}}\left(t_{k}-\tau\right)^{-\alpha_{k}\left(t_{k}\right)} \frac{\partial x}{\partial t_{k}}[\tau]_{k} d \tau
$$

6. the type III partial right Caputo derivative of order $\alpha_{k}\left(t_{k}\right)$ is defined by

$$
{ }_{t_{k}}^{C} \mathbb{D}_{b_{k}}^{\alpha_{k}\left(t_{k}\right)} x(\bar{t})=\frac{-1}{\Gamma\left(1-\alpha_{k}\left(t_{k}\right)\right)} \int_{t_{k}}^{b_{k}}\left(\tau-t_{k}\right)^{-\alpha_{k}\left(t_{k}\right)} \frac{\partial x}{\partial t_{k}}[\tau]_{k} d \tau .
$$

Similarly as done before, relations between these definitions can be proven.

Theorem 29 The following four formulas hold:

$$
\begin{aligned}
& { }_{a_{k}}^{C} D_{t_{k}}^{\alpha_{k}\left(t_{k}\right)} x(\bar{t})={ }_{a_{k}}^{C} \mathbb{D}_{t_{k}}^{\alpha_{k}\left(t_{k}\right)} x(\bar{t}) \\
& +\frac{\alpha_{k}^{\prime}\left(t_{k}\right)}{\Gamma\left(2-\alpha_{k}\left(t_{k}\right)\right)} \int_{a_{k}}^{t_{k}}\left(t_{k}-\tau\right)^{1-\alpha_{k}\left(t_{k}\right)} \frac{\partial x}{\partial t_{k}}[\tau]_{k}\left[\frac{1}{1-\alpha_{k}\left(t_{k}\right)}-\ln \left(t_{k}-\tau\right)\right] d \tau,
\end{aligned}
$$




$$
\begin{aligned}
& { }_{a_{k}}^{C} D_{t_{k}}^{\alpha_{k}\left(t_{k}\right)} x(\bar{t})={ }_{a_{k}}^{C} \mathcal{D}_{t_{k}}^{\alpha_{k}\left(t_{k}\right)} x(\bar{t}) \\
& \quad-\frac{\alpha_{k}^{\prime}\left(t_{k}\right) \Psi\left(1-\alpha_{k}\left(t_{k}\right)\right)}{\Gamma\left(1-\alpha_{k}\left(t_{k}\right)\right)} \int_{a_{k}}^{t_{k}}\left(t_{k}-\tau\right)^{-\alpha_{k}\left(t_{k}\right)}\left[x[\tau]_{k}-x\left[a_{k}\right]_{k}\right] d \tau, \quad(3.4) \\
& { }_{t_{k}}^{C} D_{b_{k}}^{\alpha_{k}\left(t_{k}\right)} x(\bar{t})={ }_{t_{k}}^{C} \mathbb{D}_{b_{k}}^{\alpha_{k}\left(t_{k}\right)} x(\bar{t}) \\
& +\frac{\alpha_{k}^{\prime}\left(t_{k}\right)}{\Gamma\left(2-\alpha_{k}\left(t_{k}\right)\right)} \int_{t_{k}}^{b_{k}}\left(\tau-t_{k}\right)^{1-\alpha_{k}\left(t_{k}\right)} \frac{\partial x}{\partial t_{k}}[\tau]_{k}\left[\frac{1}{1-\alpha_{k}\left(t_{k}\right)}-\ln \left(\tau-t_{k}\right)\right] d \tau
\end{aligned}
$$

and

$$
\begin{aligned}
{ }_{t_{k}}^{C} D_{b_{k}}^{\alpha_{k}\left(t_{k}\right)} x(\bar{t}) & ={ }_{t_{k}}^{C} \mathcal{D}_{b_{k}}^{\alpha_{k}\left(t_{k}\right)} x(\bar{t}) \\
+ & \frac{\alpha_{k}^{\prime}\left(t_{k}\right) \Psi\left(1-\alpha_{k}\left(t_{k}\right)\right)}{\Gamma\left(1-\alpha_{k}\left(t_{k}\right)\right)} \int_{t_{k}}^{b_{k}}\left(\tau-t_{k}\right)^{-\alpha_{k}\left(t_{k}\right)}\left[x[\tau]_{k}-x\left[b_{k}\right]_{k}\right] d \tau .
\end{aligned}
$$

\subsection{Numerical approximations}

Let $p \in \mathbb{N}$. We define

$$
\begin{aligned}
A_{p} & =\frac{1}{\Gamma\left(p+1-\alpha_{k}\left(t_{k}\right)\right)}\left[1+\sum_{l=n-p+1}^{N} \frac{\Gamma\left(\alpha_{k}\left(t_{k}\right)-n+l\right)}{\Gamma\left(\alpha_{k}\left(t_{k}\right)-p\right)(l-n+p) !}\right], \\
B_{p} & =\frac{\Gamma\left(\alpha_{k}\left(t_{k}\right)-n+p\right)}{\Gamma\left(1-\alpha_{k}\left(t_{k}\right)\right) \Gamma\left(\alpha_{k}\left(t_{k}\right)\right)(p-n) !}, \\
V_{p}(\bar{t}) & =\int_{a_{k}}^{t_{k}}\left(\tau-a_{k}\right)^{p-n} \frac{\partial x}{\partial t_{k}}[\tau]_{k} d \tau, \\
L_{p}(\bar{t}) & =\max _{\tau \in\left[a_{k}, t_{k}\right]}\left|\frac{\partial^{p} x}{\partial t_{k}^{p}}[\tau]_{k}\right| .
\end{aligned}
$$

Theorem 30 Let $x \in C^{n+1}\left(\prod_{i=1}^{m}\left[a_{i}, b_{i}\right], \mathbb{R}\right)$ with $n \in \mathbb{N}$. Then, for all $k \in$ $\{1, \ldots, m\}$ and for all $N \in \mathbb{N}$ such that $N \geq n$, we have

$$
\begin{aligned}
{ }_{a_{k}}^{C} \mathbb{D}_{t_{k}}^{\alpha_{k}\left(t_{k}\right)} x(\bar{t})= & \sum_{p=1}^{n} A_{p}\left(t_{k}-a_{k}\right)^{p-\alpha_{k}\left(t_{k}\right)} \frac{\partial^{p} x}{\partial t_{k}^{p}}\left[t_{k}\right]_{k} \\
& +\sum_{p=n}^{N} B_{p}\left(t_{k}-a_{k}\right)^{n-p-\alpha_{k}\left(t_{k}\right)} V_{p}(\bar{t})+E(\bar{t}) .
\end{aligned}
$$

The approximation error $E(\bar{t})$ is bounded by

$$
E(\bar{t}) \leq L_{n+1}(\bar{t}) \frac{\exp \left(\left(n-\alpha_{k}\left(t_{k}\right)\right)^{2}+n-\alpha_{k}\left(t_{k}\right)\right)}{\Gamma\left(n+1-\alpha_{k}\left(t_{k}\right)\right) N^{n-\alpha_{k}\left(t_{k}\right)}\left(n-\alpha_{k}\left(t_{k}\right)\right)}\left(t_{k}-a_{k}\right)^{n+1-\alpha_{k}\left(t_{k}\right)} .
$$


Proof. By definition,

$$
{ }_{a_{k}}^{C} \mathbb{D}_{t_{k}}^{\alpha_{k}\left(t_{k}\right)} x(\bar{t})=\frac{1}{\Gamma\left(1-\alpha_{k}\left(t_{k}\right)\right)} \int_{a_{k}}^{t_{k}}\left(t_{k}-\tau\right)^{-\alpha_{k}\left(t_{k}\right)} \frac{\partial x}{\partial t_{k}}[\tau]_{k} d \tau
$$

and, integrating by parts with $u^{\prime}(\tau)=\left(t_{k}-\tau\right)^{-\alpha_{k}\left(t_{k}\right)}$ and $v(\tau)=\frac{\partial x}{\partial t_{k}}[\tau]_{k}$, we deduce that

$$
\begin{aligned}
{ }_{a_{k}}^{C} \mathbb{D}_{t_{k}}^{\alpha_{k}\left(t_{k}\right)} x(\bar{t})= & \frac{\left(t_{k}-a_{k}\right)^{1-\alpha_{k}\left(t_{k}\right)}}{\Gamma\left(2-\alpha_{k}\left(t_{k}\right)\right)} \frac{\partial x}{\partial t_{k}}\left[a_{k}\right]_{k} \\
& +\frac{1}{\Gamma\left(2-\alpha_{k}\left(t_{k}\right)\right)} \int_{a_{k}}^{t_{k}}\left(t_{k}-\tau\right)^{1-\alpha_{k}\left(t_{k}\right)} \frac{\partial^{2} x}{\partial t_{k}^{2}}[\tau]_{k} d \tau .
\end{aligned}
$$

Integrating again by parts, taking $u^{\prime}(\tau)=\left(t_{k}-\tau\right)^{1-\alpha_{k}\left(t_{k}\right)}$ and $v(\tau)=\frac{\partial^{2} x}{\partial t_{k}^{2}}[\tau]_{k}$, we get

$$
\begin{array}{r}
{ }_{a_{k}}^{C} \mathbb{D}_{t_{k}}^{\alpha_{k}\left(t_{k}\right)} x(\bar{t})=\frac{\left(t_{k}-a_{k}\right)^{1-\alpha_{k}\left(t_{k}\right)}}{\Gamma\left(2-\alpha_{k}\left(t_{k}\right)\right)} \frac{\partial x}{\partial t_{k}}\left[a_{k}\right]_{k}+\frac{\left(t_{k}-a_{k}\right)^{2-\alpha_{k}\left(t_{k}\right)}}{\Gamma\left(3-\alpha_{k}\left(t_{k}\right)\right)} \frac{\partial^{2} x}{\partial t_{k}^{2}}\left[a_{k}\right]_{k} \\
+\frac{1}{\Gamma\left(3-\alpha_{k}\left(t_{k}\right)\right)} \int_{a_{k}}^{t_{k}}\left(t_{k}-\tau\right)^{2-\alpha_{k}\left(t_{k}\right)} \frac{\partial^{3} x}{\partial t_{k}^{3}}[\tau]_{k} d \tau .
\end{array}
$$

Repeating the same procedure $n-2$ more times, we get the expansion formula

$$
\begin{aligned}
{ }_{a_{k}}^{C} \mathbb{D}_{t_{k}}^{\alpha_{k}\left(t_{k}\right)} x(\bar{t})= & \sum_{p=1}^{n} \frac{\left(t_{k}-a_{k}\right)^{p-\alpha_{k}\left(t_{k}\right)}}{\Gamma\left(p+1-\alpha_{k}\left(t_{k}\right)\right)} \frac{\partial^{p} x}{\partial t_{k}^{p}}\left[a_{k}\right]_{k} \\
& +\frac{1}{\Gamma\left(n+1-\alpha_{k}\left(t_{k}\right)\right)} \int_{a_{k}}^{t_{k}}\left(t_{k}-\tau\right)^{n-\alpha_{k}\left(t_{k}\right)} \frac{\partial^{n+1} x}{\partial t_{k}^{n+1}}[\tau]_{k} d \tau .
\end{aligned}
$$

Using the equalities

$$
\begin{aligned}
\left(t_{k}-\tau\right)^{n-\alpha_{k}\left(t_{k}\right)} & =\left(t_{k}-a_{k}\right)^{n-\alpha_{k}\left(t_{k}\right)}\left(1-\frac{\tau-a_{k}}{t_{k}-a_{k}}\right)^{n-\alpha_{k}\left(t_{k}\right)} \\
& =\left(t_{k}-a_{k}\right)^{n-\alpha_{k}\left(t_{k}\right)}\left[\sum_{p=0}^{N}\left(\begin{array}{c}
n-\alpha_{k}\left(t_{k}\right) \\
p
\end{array}\right)(-1)^{p} \frac{\left(\tau-a_{k}\right)^{p}}{\left(t_{k}-a_{k}\right)^{p}}+\bar{E}(\bar{t})\right]
\end{aligned}
$$

with

$$
\bar{E}(\bar{t})=\sum_{p=N+1}^{\infty}\left(\begin{array}{c}
n-\alpha_{k}\left(t_{k}\right) \\
p
\end{array}\right)(-1)^{p} \frac{\left(\tau-a_{k}\right)^{p}}{\left(t_{k}-a_{k}\right)^{p}},
$$

we arrive at 


$$
\begin{aligned}
& { }_{a_{k}}^{C} \mathbb{D}_{t_{k}}^{\alpha_{k}\left(t_{k}\right)} x(\bar{t})=\sum_{p=1}^{n} \frac{\left(t_{k}-a_{k}\right)^{p-\alpha_{k}\left(t_{k}\right)}}{\Gamma\left(p+1-\alpha_{k}\left(t_{k}\right)\right)} \frac{\partial^{p} x}{\partial t_{k}^{p}}\left[a_{k}\right]_{k} \\
& +\frac{\left(t_{k}-a_{k}\right)^{n-\alpha_{k}\left(t_{k}\right)}}{\Gamma\left(n+1-\alpha_{k}\left(t_{k}\right)\right)} \int_{a_{k}}^{t_{k}} \sum_{p=0}^{N}\left(\begin{array}{c}
n-\alpha_{k}\left(t_{k}\right) \\
p
\end{array}\right)(-1)^{p} \frac{\left(\tau-a_{k}\right)^{p}}{\left(t_{k}-a_{k}\right)^{p}} \frac{\partial^{n+1} x}{\partial t_{k}^{n+1}}[\tau]_{k} d \tau+E(\bar{t}) \\
& =\sum_{p=1}^{n} \frac{\left(t_{k}-a_{k}\right)^{p-\alpha_{k}\left(t_{k}\right)}}{\Gamma\left(p+1-\alpha_{k}\left(t_{k}\right)\right)} \frac{\partial^{p} x}{\partial t_{k}^{p}}\left[a_{k}\right]_{k}+\frac{\left(t_{k}-a_{k}\right)^{n-\alpha_{k}\left(t_{k}\right)}}{\Gamma\left(n+1-\alpha_{k}\left(t_{k}\right)\right)} \\
& \times \sum_{p=0}^{N}\left(\begin{array}{c}
n-\alpha_{k}\left(t_{k}\right) \\
p
\end{array}\right) \frac{(-1)^{p}}{\left(t_{k}-a_{k}\right)^{p}} \int_{a_{k}}^{t_{k}}\left(\tau-a_{k}\right)^{p} \frac{\partial^{n+1} x}{\partial t_{k}^{n+1}}[\tau]_{k} d \tau+E(\bar{t})
\end{aligned}
$$

with

$$
E(\bar{t})=\frac{\left(t_{k}-a_{k}\right)^{n-\alpha_{k}\left(t_{k}\right)}}{\Gamma\left(n+1-\alpha_{k}\left(t_{k}\right)\right)} \int_{a_{k}}^{t_{k}} \bar{E}(\bar{t}) \frac{\partial^{n+1} x}{\partial t_{k}^{n+1}}[\tau]_{k} d \tau .
$$

Now, we split the last sum into $p=0$ and the remaining terms $p=1, \ldots, N$ and integrate by parts with $u(\tau)=\left(\tau-a_{k}\right)^{p}$ and $v^{\prime}(\tau)=\frac{\partial^{n+1} x}{\partial t_{k}^{n+1}}[\tau]_{k}$. Observing that

$$
\left(\begin{array}{c}
n-\alpha_{k}\left(t_{k}\right) \\
p
\end{array}\right)(-1)^{p}=\frac{\Gamma\left(\alpha_{k}\left(t_{k}\right)-n+p\right)}{\Gamma\left(\alpha_{k}\left(t_{k}\right)-n\right) p !}
$$

we obtain:

$$
\begin{aligned}
& \frac{\left(t_{k}-a_{k}\right)^{n-\alpha_{k}\left(t_{k}\right)}}{\Gamma\left(n+1-\alpha_{k}\left(t_{k}\right)\right)} \sum_{p=0}^{N}\left(\begin{array}{c}
n-\alpha_{k}\left(t_{k}\right) \\
p
\end{array}\right) \frac{(-1)^{p}}{\left(t_{k}-a_{k}\right)^{p}} \int_{a_{k}}^{t_{k}}\left(\tau-a_{k}\right)^{p} \frac{\partial^{n+1} x}{\partial t_{k}^{n+1}}[\tau]_{k} d \tau \\
& =\frac{\left(t_{k}-a_{k}\right)^{n-\alpha_{k}\left(t_{k}\right)}}{\Gamma\left(n+1-\alpha_{k}\left(t_{k}\right)\right)}\left[\frac{\partial^{n} x}{\partial t_{k}^{n}}\left[t_{k}\right]_{k}-\frac{\partial^{n} x}{\partial t_{k}^{n}}\left[a_{k}\right]_{k}\right] \\
& +\frac{\left(t_{k}-a_{k}\right)^{n-\alpha_{k}\left(t_{k}\right)}}{\Gamma\left(n+1-\alpha_{k}\left(t_{k}\right)\right)} \sum_{p=1}^{N} \frac{\Gamma\left(\alpha_{k}\left(t_{k}\right)-n+p\right)}{\Gamma\left(\alpha_{k}\left(t_{k}\right)-n\right) p !\left(t_{k}-a_{k}\right)^{p}} \\
& \times\left[\left(t_{k}-a_{k}\right)^{p} \frac{\partial^{n} x}{\partial t_{k}^{n}}\left[t_{k}\right]_{k}-\int_{a_{k}}^{t_{k}} p\left(\tau-a_{k}\right)^{p-1} \frac{\partial^{n} x}{\partial t_{k}^{n}}[\tau]_{k} d \tau\right] \\
& =-\frac{\left(t_{k}-a_{k}\right)^{n-\alpha_{k}\left(t_{k}\right)}}{\Gamma\left(n+1-\alpha_{k}\left(t_{k}\right)\right)} \frac{\partial^{n} x}{\partial t_{k}^{n}}\left[a_{k}\right]_{k}+\frac{\left(t_{k}-a_{k}\right)^{n-\alpha_{k}\left(t_{k}\right)}}{\Gamma\left(n+1-\alpha_{k}\left(t_{k}\right)\right)} \frac{\partial^{n} x}{\partial t_{k}^{n}}\left[t_{k}\right]_{k} \\
& \times\left[1+\sum_{p=1}^{N} \frac{\Gamma\left(\alpha_{k}\left(t_{k}\right)-n+p\right)}{\Gamma\left(\alpha_{k}\left(t_{k}\right)-n\right) p !}\right]+\frac{\left(t_{k}-a_{k}\right)^{n-\alpha_{k}\left(t_{k}\right)-1}}{\Gamma\left(n-\alpha_{k}\left(t_{k}\right)\right)} \\
& \times \sum_{p=1}^{N} \frac{\Gamma\left(\alpha_{k}\left(t_{k}\right)-n+p\right)}{\Gamma\left(\alpha_{k}\left(t_{k}\right)+1-n\right)(p-1) !\left(t_{k}-a_{k}\right)^{p-1}} \int_{a_{k}}^{t_{k}}\left(\tau-a_{k}\right)^{p-1} \frac{\partial^{n} x}{\partial t_{k}^{n}}[\tau]_{k} d \tau .
\end{aligned}
$$

Thus, we get 


$$
\begin{aligned}
{ }_{a_{k}}^{C} \mathbb{D}_{t_{k}}^{\alpha_{k}\left(t_{k}\right)} & x(\bar{t})=\sum_{p=1}^{n-1} \frac{\left(t_{k}-a_{k}\right)^{p-\alpha_{k}\left(t_{k}\right)}}{\Gamma\left(p+1-\alpha_{k}\left(t_{k}\right)\right)} \frac{\partial^{p} x}{\partial t_{k}^{p}}\left[a_{k}\right]_{k} \\
& +\frac{\left(t_{k}-a_{k}\right)^{n-\alpha_{k}\left(t_{k}\right)}}{\Gamma\left(n+1-\alpha_{k}\left(t_{k}\right)\right)} \frac{\partial^{n} x}{\partial t_{k}^{n}}\left[t_{k}\right]_{k}\left[1+\sum_{p=1}^{N} \frac{\Gamma\left(\alpha_{k}\left(t_{k}\right)-n+p\right)}{\Gamma\left(\alpha_{k}\left(t_{k}\right)-n\right) p !}\right] \\
& +\frac{\left(t_{k}-a_{k}\right)^{n-\alpha_{k}\left(t_{k}\right)-1}}{\Gamma\left(n-\alpha_{k}\left(t_{k}\right)\right)} \sum_{p=1}^{N} \frac{\Gamma\left(\alpha_{k}\left(t_{k}\right)-n+p\right)}{\Gamma\left(\alpha_{k}\left(t_{k}\right)+1-n\right)(p-1) !\left(t_{k}-a_{k}\right)^{p-1}} \\
& \times \int_{a_{k}}^{t_{k}}\left(\tau-a_{k}\right)^{p-1} \frac{\partial^{n} x}{\partial t_{k}^{n}}[\tau]_{k} d \tau+E(\bar{t}) .
\end{aligned}
$$

Repeating the process $n-1$ more times with respect to the last sum, that is, splitting the first term of the sum and integrating by parts the obtained result, we arrive to

$$
\begin{aligned}
{ }_{a_{k}}^{C} \mathbb{D}_{t_{k}}^{\alpha_{k}\left(t_{k}\right)} x(\bar{t})= & \sum_{p=1}^{n} \frac{\left(t_{k}-a_{k}\right)^{p-\alpha_{k}\left(t_{k}\right)}}{\Gamma\left(p+1-\alpha_{k}\left(t_{k}\right)\right)} \frac{\partial^{p} x}{\partial t_{k}^{p}}\left[t_{k}\right]_{k} \\
& \times\left[1+\sum_{l=n-p+1}^{N} \frac{\Gamma\left(\alpha_{k}\left(t_{k}\right)-n+l\right)}{\Gamma\left(\alpha_{k}\left(t_{k}\right)-p\right)(l-n+p) !}\right] \\
& +\sum_{p=n}^{N} \frac{\Gamma\left(\alpha_{k}\left(t_{k}\right)-n+p\right)}{\Gamma\left(1-\alpha_{k}\left(t_{k}\right)\right) \Gamma\left(\alpha_{k}\left(t_{k}\right)\right)(p-n) !}\left(t_{k}-a_{k}\right)^{n-p-\alpha_{k}\left(t_{k}\right)} \\
& \times \int_{a_{k}}^{t_{k}}\left(\tau-a_{k}\right)^{p-n} \frac{\partial x}{\partial t_{k}}[\tau]_{k} d \tau+E(\bar{t}) .
\end{aligned}
$$

We now seek the upper bound formula for $E(\bar{t})$. Using the two relations

$$
\left|\frac{\tau-a_{k}}{t_{k}-a_{k}}\right| \leq 1, \text { if } \tau \in\left[a_{k}, t_{k}\right]
$$

and

$$
\left|\left(\begin{array}{c}
n-\alpha_{k}\left(t_{k}\right) \\
p
\end{array}\right)\right| \leq \frac{\exp \left(\left(n-\alpha_{k}\left(t_{k}\right)\right)^{2}+n-\alpha_{k}\left(t_{k}\right)\right)}{p^{n+1-\alpha_{k}\left(t_{k}\right)}},
$$

we get

$$
\begin{aligned}
\bar{E}(\bar{t}) & \leq \sum_{p=N+1}^{\infty} \frac{\exp \left(\left(n-\alpha_{k}\left(t_{k}\right)\right)^{2}+n-\alpha_{k}\left(t_{k}\right)\right)}{p^{n+1-\alpha_{k}\left(t_{k}\right)}} \\
& \leq \int_{N}^{\infty} \frac{\exp \left(\left(n-\alpha_{k}\left(t_{k}\right)\right)^{2}+n-\alpha_{k}\left(t_{k}\right)\right)}{p^{n+1-\alpha_{k}\left(t_{k}\right)}} d p \\
& =\frac{\exp \left(\left(n-\alpha_{k}\left(t_{k}\right)\right)^{2}+n-\alpha_{k}\left(t_{k}\right)\right)}{N^{n-\alpha_{k}\left(t_{k}\right)}\left(n-\alpha_{k}\left(t_{k}\right)\right)} .
\end{aligned}
$$

Then, 
$E(\bar{t}) \leq L_{n+1}(\bar{t}) \frac{\exp \left(\left(n-\alpha_{k}\left(t_{k}\right)\right)^{2}+n-\alpha_{k}\left(t_{k}\right)\right)}{\Gamma\left(n+1-\alpha_{k}\left(t_{k}\right)\right) N^{n-\alpha_{k}\left(t_{k}\right)}\left(n-\alpha_{k}\left(t_{k}\right)\right)}\left(t_{k}-a_{k}\right)^{n+1-\alpha_{k}\left(t_{k}\right)}$.

This concludes the proof.

Remark 31 In Theorem 30 we have

$$
\lim _{N \rightarrow \infty} E(\bar{t})=0
$$

for all $\bar{t} \in \prod_{i=1}^{m}\left[a_{i}, b_{i}\right]$ and $n \in \mathbb{N}$.

Theorem 32 Let $x \in C^{n+1}\left(\prod_{i=1}^{m}\left[a_{i}, b_{i}\right], \mathbb{R}\right)$ with $n \in \mathbb{N}$. Then, for all $k \in$ $\{1, \ldots, m\}$ and for all $N \in \mathbb{N}$ such that $N \geq n$, we have

$$
\begin{aligned}
{ }_{a_{k}}^{C} D_{t_{k}}^{\alpha_{k}\left(t_{k}\right)} x(\bar{t}) & =\sum_{p=1}^{n} A_{p}\left(t_{k}-a_{k}\right)^{p-\alpha_{k}\left(t_{k}\right)} \frac{\partial^{p} x}{\partial t_{k}^{p}}\left[t_{k}\right]_{k} \\
+ & \sum_{p=n}^{N} B_{p}\left(t_{k}-a_{k}\right)^{n-p-\alpha_{k}\left(t_{k}\right)} V_{p}(\bar{t})+\frac{\alpha_{k}^{\prime}\left(t_{k}\right)\left(t_{k}-a_{k}\right)^{1-\alpha_{k}\left(t_{k}\right)}}{\Gamma\left(2-\alpha_{k}\left(t_{k}\right)\right)} \\
\times & {\left[\left(\frac{1}{1-\alpha_{k}\left(t_{k}\right)}-\ln \left(t_{k}-a_{k}\right)\right) \sum_{p=0}^{N}\left(\begin{array}{c}
1-\alpha_{k}\left(t_{k}\right) \\
p
\end{array}\right) \frac{(-1)^{p}}{\left(t_{k}-a_{k}\right)^{p}} V_{n+p}(\bar{t})\right.} \\
+ & \left.\sum_{p=0}^{N}\left(\begin{array}{c}
1-\alpha_{k}\left(t_{k}\right) \\
p
\end{array}\right)(-1)^{p} \sum_{r=1}^{N} \frac{1}{r\left(t_{k}-a_{k}\right)^{p+r}} V_{n+p+r}(\bar{t})\right]+E(\bar{t}) .
\end{aligned}
$$

The approximation error $E(\bar{t})$ is bounded by

$$
\begin{aligned}
E(\bar{t}) \leq & L_{n+1}(\bar{t}) \frac{\exp \left(\left(n-\alpha_{k}\left(t_{k}\right)\right)^{2}+n-\alpha_{k}\left(t_{k}\right)\right)}{\Gamma\left(n+1-\alpha_{k}\left(t_{k}\right)\right) N^{n-\alpha_{k}\left(t_{k}\right)}\left(n-\alpha_{k}\left(t_{k}\right)\right)}\left(t_{k}-a_{k}\right)^{n+1-\alpha_{k}\left(t_{k}\right)} \\
& +\left|\alpha_{k}^{\prime}\left(t_{k}\right)\right| L_{1}(\bar{t}) \frac{\exp \left(\left(1-\alpha_{k}\left(t_{k}\right)\right)^{2}+1-\alpha_{k}\left(t_{k}\right)\right)}{\Gamma\left(2-\alpha_{k}\left(t_{k}\right)\right) N^{1-\alpha_{k}\left(t_{k}\right)}\left(1-\alpha_{k}\left(t_{k}\right)\right)} \\
& \times\left[\left|\frac{1}{1-\alpha_{k}\left(t_{k}\right)}-\ln \left(t_{k}-a_{k}\right)\right|+\frac{1}{N}\right]\left(t_{k}-a_{k}\right)^{2-\alpha_{k}\left(t_{k}\right)} .
\end{aligned}
$$

Proof. Taking into account relation (3.3) and Theorem 30, we only need to expand the term

$$
\frac{\alpha_{k}^{\prime}\left(t_{k}\right)}{\Gamma\left(2-\alpha_{k}\left(t_{k}\right)\right)} \int_{a_{k}}^{t_{k}}\left(t_{k}-\tau\right)^{1-\alpha_{k}\left(t_{k}\right)} \frac{\partial x}{\partial t_{k}}[\tau]_{k}\left[\frac{1}{1-\alpha_{k}\left(t_{k}\right)}-\ln \left(t_{k}-\tau\right)\right] d \tau .
$$

Splitting the integral, and using the expansion formulas

$$
\begin{aligned}
\left(t_{k}-\tau\right)^{1-\alpha_{k}\left(t_{k}\right)} & =\left(t_{k}-a_{k}\right)^{1-\alpha_{k}\left(t_{k}\right)}\left(1-\frac{\tau-a_{k}}{t_{k}-a_{k}}\right)^{1-\alpha_{k}\left(t_{k}\right)} \\
& =\left(t_{k}-a_{k}\right)^{1-\alpha_{k}\left(t_{k}\right)}\left[\sum_{p=0}^{N}\left(\begin{array}{c}
1-\alpha_{k}\left(t_{k}\right) \\
p
\end{array}\right)(-1)^{p} \frac{\left(\tau-a_{k}\right)^{p}}{\left(t_{k}-a_{k}\right)^{p}}+\bar{E}_{1}(\bar{t})\right]
\end{aligned}
$$


with

$$
\bar{E}_{1}(\bar{t})=\sum_{p=N+1}^{\infty}\left(\begin{array}{c}
1-\alpha_{k}\left(t_{k}\right) \\
p
\end{array}\right)(-1)^{p} \frac{\left(\tau-a_{k}\right)^{p}}{\left(t_{k}-a_{k}\right)^{p}}
$$

and

$$
\begin{aligned}
\ln \left(t_{k}-\tau\right) & =\ln \left(t_{k}-a_{k}\right)+\ln \left(1-\frac{\tau-a_{k}}{t_{k}-a_{k}}\right) \\
& =\ln \left(t_{k}-a_{k}\right)-\sum_{r=1}^{N} \frac{1}{r} \frac{\left(\tau-a_{k}\right)^{r}}{\left(t_{k}-a_{k}\right)^{r}}-\bar{E}_{2}(\bar{t})
\end{aligned}
$$

with

$$
\bar{E}_{2}(\bar{t})=\sum_{r=N+1}^{\infty} \frac{1}{r} \frac{\left(\tau-a_{k}\right)^{r}}{\left(t_{k}-a_{k}\right)^{r}},
$$

we conclude that (3.5) is equivalent to

$$
\begin{aligned}
& \frac{\alpha_{k}^{\prime}\left(t_{k}\right)}{\Gamma\left(2-\alpha_{k}\left(t_{k}\right)\right)}\left[\left(\frac{1}{1-\alpha_{k}\left(t_{k}\right)}-\ln \left(t_{k}-a_{k}\right)\right) \int_{a_{k}}^{t_{k}}\left(t_{k}-\tau\right)^{1-\alpha_{k}\left(t_{k}\right)} \frac{\partial x}{\partial t_{k}}[\tau]_{k} d \tau\right. \\
& \left.\quad-\int_{a_{k}}^{t_{k}}\left(t_{k}-\tau\right)^{1-\alpha_{k}\left(t_{k}\right)} \ln \left(1-\frac{\tau-a_{k}}{t_{k}-a_{k}}\right) \frac{\partial x}{\partial t_{k}}[\tau]_{k} d \tau\right] \\
& =\frac{\alpha_{k}^{\prime}\left(t_{k}\right)}{\Gamma\left(2-\alpha_{k}\left(t_{k}\right)\right)}\left[\left(\frac{1}{1-\alpha_{k}\left(t_{k}\right)}-\ln \left(t_{k}-a_{k}\right)\right)\right. \\
& \quad \times \int_{a_{k}}^{t_{k}}\left(t_{k}-a_{k}\right)^{1-\alpha_{k}\left(t_{k}\right)} \sum_{p=0}^{N}\left(1-\alpha_{k}\left(t_{k}\right)\right)(-1)^{p} \frac{\left(\tau-a_{k}\right)^{p}}{\left(t_{k}-a_{k}\right)^{p}} \frac{\partial x}{\partial t_{k}}[\tau]_{k} d \tau \\
& \quad+\int_{a_{k}}^{t_{k}}\left(t_{k}-a_{k}\right)^{1-\alpha_{k}\left(t_{k}\right)} \sum_{p=0}^{N}\left(1-\alpha_{k}\left(t_{k}\right)\right)(-1)^{p} \frac{\left(\tau-a_{k}\right)^{p}}{\left(t_{k}-a_{k}\right)^{p}} \\
& \left.\quad \times \sum_{r=1}^{N} \frac{1}{r} \frac{\left(\tau-a_{k}\right)^{r}}{\left(t_{k}-a_{k}\right)^{r}} \frac{\partial x}{\partial t_{k}}[\tau]_{k} d \tau\right]+\frac{\alpha_{k}^{\prime}\left(t_{k}\right)}{\Gamma\left(2-\alpha_{k}\left(t_{k}\right)\right)}\left[\left(\frac{1}{1-\alpha_{k}\left(t_{k}\right)}-\ln \left(t_{k}-a_{k}\right)\right)\right. \\
& \quad \times \int_{a_{k}}^{t_{k}}\left(t_{k}-a_{k}\right)^{1-\alpha_{k}\left(t_{k}\right)} \bar{E}_{1}(\bar{t}) \frac{\partial x}{\partial t_{k}}[\tau]_{k} d \tau \\
& \left.\quad+\int_{a_{k}}^{t_{k}}\left(t_{k}-a_{k}\right)^{1-\alpha_{k}\left(t_{k}\right)} \bar{E}_{1}(\bar{t}) \bar{E}_{2}(\bar{t}) \frac{\partial x}{\partial t_{k}}[\tau]_{k} d \tau\right] \\
& =\frac{\alpha_{k}^{\prime}\left(t_{k}\right)\left(t_{k}-a_{k}\right)^{1-\alpha_{k}\left(t_{k}\right)}}{\Gamma\left(2-\alpha_{k}\left(t_{k}\right)\right)}\left[\left(\frac{1}{1-\alpha_{k}\left(t_{k}\right)}-\ln \left(t_{k}-a_{k}\right)\right) \sum_{p=0}^{N}\left(1-\alpha_{k}\left(t_{k}\right)\right)\right. \\
& \left.\quad \times \frac{(-1)^{p}}{\left(t_{k}-a_{k}\right)^{p}} V_{n+p}(\bar{t})+\sum_{p=0}^{N}\left(1-\alpha_{k}\left(t_{k}\right)\right)(-1)^{p} \sum_{r=1}^{N} \frac{1}{r\left(t_{k}-a_{k}\right)^{p+r}} V_{n+p+r}(\bar{t})\right] \\
&
\end{aligned}
$$




$$
\begin{aligned}
& +\frac{\alpha_{k}^{\prime}\left(t_{k}\right)\left(t_{k}-a_{k}\right)^{1-\alpha_{k}\left(t_{k}\right)}}{\Gamma\left(2-\alpha_{k}\left(t_{k}\right)\right)}\left[\left(\frac{1}{1-\alpha_{k}\left(t_{k}\right)}-\ln \left(t_{k}-a_{k}\right)\right)\right. \\
& \left.\times \int_{a_{k}}^{t_{k}} \bar{E}_{1}(\bar{t}) \frac{\partial x}{\partial t_{k}}[\tau]_{k} d \tau+\int_{a_{k}}^{t_{k}} \bar{E}_{1}(\bar{t}) \bar{E}_{2}(\bar{t}) \frac{\partial x}{\partial t_{k}}[\tau]_{k} d \tau\right] .
\end{aligned}
$$

For the error analysis, we know from Theorem 30 that

$$
\bar{E}_{1}(\bar{t}) \leq \frac{\exp \left(\left(1-\alpha_{k}\left(t_{k}\right)\right)^{2}+1-\alpha_{k}\left(t_{k}\right)\right)}{N^{1-\alpha_{k}\left(t_{k}\right)}\left(1-\alpha_{k}\left(t_{k}\right)\right)} .
$$

Then,

$$
\begin{aligned}
& \left|\int_{a_{k}}^{t_{k}}\left(t_{k}-a_{k}\right)^{1-\alpha_{k}\left(t_{k}\right)} \bar{E}_{1}(\bar{t}) \frac{\partial x}{\partial t_{k}}[\tau]_{k} d \tau\right| \\
& \quad \leq L_{1}(\bar{t}) \frac{\exp \left(\left(1-\alpha_{k}\left(t_{k}\right)\right)^{2}+1-\alpha_{k}\left(t_{k}\right)\right)}{N^{1-\alpha_{k}\left(t_{k}\right)}\left(1-\alpha_{k}\left(t_{k}\right)\right)}\left(t_{k}-a_{k}\right)^{2-\alpha_{k}\left(t_{k}\right)} .
\end{aligned}
$$

On the other hand, we have

$$
\begin{aligned}
& \left|\int_{a_{k}}^{t_{k}}\left(t_{k}-a_{k}\right)^{1-\alpha_{k}\left(t_{k}\right)} \bar{E}_{1}(\bar{t}) \bar{E}_{2}(\bar{t}) \frac{\partial x}{\partial t_{k}}[\tau]_{k} d \tau\right| \\
& \leq L_{1}(\bar{t}) \frac{\exp \left(\left(1-\alpha_{k}\left(t_{k}\right)\right)^{2}+1-\alpha_{k}\left(t_{k}\right)\right)}{N^{1-\alpha_{k}\left(t_{k}\right)}\left(1-\alpha_{k}\left(t_{k}\right)\right)}\left(t_{k}-a_{k}\right)^{1-\alpha_{k}\left(t_{k}\right)} \\
& \quad \times \sum_{r=N+1}^{\infty} \frac{1}{r\left(t_{k}-a_{k}\right)^{r}} \int_{a_{k}}^{t_{k}}\left(\tau-a_{k}\right)^{r} d \tau \\
& =L_{1}(\bar{t}) \frac{\exp \left(\left(1-\alpha_{k}\left(t_{k}\right)\right)^{2}+1-\alpha_{k}\left(t_{k}\right)\right)}{N^{1-\alpha_{k}\left(t_{k}\right)}\left(1-\alpha_{k}\left(t_{k}\right)\right)}\left(t_{k}-a_{k}\right)^{1-\alpha_{k}\left(t_{k}\right)} \sum_{r=N+1}^{\infty} \frac{t_{k}-a_{k}}{r(r+1)} \\
& \leq L_{1}(\bar{t}) \frac{\exp \left(\left(1-\alpha_{k}\left(t_{k}\right)\right)^{2}+1-\alpha_{k}\left(t_{k}\right)\right)}{N^{2-\alpha_{k}\left(t_{k}\right)}\left(1-\alpha_{k}\left(t_{k}\right)\right)}\left(t_{k}-a_{k}\right)^{2-\alpha_{k}\left(t_{k}\right)} .
\end{aligned}
$$

We get the desired result by combining inequalities (3.6) and (3.7).

Theorem 33 Let $x \in C^{n+1}\left(\prod_{i=1}^{m}\left[a_{i}, b_{i}\right], \mathbb{R}\right)$ with $n \in \mathbb{N}$. Then, for all $k \in$ $\{1, \ldots, m\}$ and for all $N \in \mathbb{N}$ such that $N \geq n$, we have

$$
\begin{aligned}
{ }_{a_{k}}^{C} \mathcal{D}_{t_{k}}^{\alpha_{k}\left(t_{k}\right)} x(\bar{t})=\sum_{p=1}^{n} A_{p}\left(t_{k}-a_{k}\right)^{p-\alpha_{k}\left(t_{k}\right)} \frac{\partial^{p} x}{\partial t_{k}^{p}}\left[t_{k}\right]_{k} \\
\quad+\sum_{p=n}^{N} B_{p}\left(t_{k}-a_{k}\right)^{n-p-\alpha_{k}\left(t_{k}\right)} V_{p}(\bar{t})+\frac{\alpha_{k}^{\prime}\left(t_{k}\right)\left(t_{k}-a_{k}\right)^{1-\alpha_{k}\left(t_{k}\right)}}{\Gamma\left(2-\alpha_{k}\left(t_{k}\right)\right)} \\
\times\left[\left(\Psi\left(2-\alpha_{k}\left(t_{k}\right)\right)-\ln \left(t_{k}-a_{k}\right)\right) \sum_{p=0}^{N}\left(\begin{array}{c}
1-\alpha_{k}\left(t_{k}\right) \\
p
\end{array}\right) \frac{(-1)^{p}}{\left(t_{k}-a_{k}\right)^{p}} V_{n+p}(\bar{t})\right. \\
\left.+\sum_{p=0}^{N}\left(\begin{array}{c}
1-\alpha_{k}\left(t_{k}\right) \\
p
\end{array}\right)(-1)^{p} \sum_{r=1}^{N} \frac{1}{r\left(t_{k}-a_{k}\right)^{p+r}} V_{n+p+r}(\bar{t})\right]+E(\bar{t}) .
\end{aligned}
$$


The approximation error $E(\bar{t})$ is bounded by

$$
\begin{aligned}
E(\bar{t}) \leq & L_{n+1}(\bar{t}) \frac{\exp \left(\left(n-\alpha_{k}\left(t_{k}\right)\right)^{2}+n-\alpha_{k}\left(t_{k}\right)\right)}{\Gamma\left(n+1-\alpha_{k}\left(t_{k}\right)\right) N^{n-\alpha_{k}\left(t_{k}\right)}\left(n-\alpha_{k}\left(t_{k}\right)\right)}\left(t_{k}-a_{k}\right)^{n+1-\alpha_{k}\left(t_{k}\right)} \\
& +\left|\alpha_{k}^{\prime}\left(t_{k}\right)\right| L_{1}(\bar{t}) \frac{\exp \left(\left(1-\alpha_{k}\left(t_{k}\right)\right)^{2}+1-\alpha_{k}\left(t_{k}\right)\right)}{\Gamma\left(2-\alpha_{k}\left(t_{k}\right)\right) N^{1-\alpha_{k}\left(t_{k}\right)}\left(1-\alpha_{k}\left(t_{k}\right)\right)} \\
& \times\left[\left|\Psi\left(2-\alpha_{k}\left(t_{k}\right)\right)-\ln \left(t_{k}-a_{k}\right)\right|+\frac{1}{N}\right]\left(t_{k}-a_{k}\right)^{2-\alpha_{k}\left(t_{k}\right)} .
\end{aligned}
$$

Proof. Starting with relation (3.4), and integrating by parts the integral, we obtain that

$$
\begin{aligned}
{ }_{a_{k}}^{C} \mathcal{D}_{t_{k}}^{\alpha_{k}\left(t_{k}\right)} x(\bar{t})={ }_{a_{k}}^{C} D_{t_{k}}^{\alpha_{k}\left(t_{k}\right)} x(\bar{t}) \\
\quad+\frac{\alpha_{k}^{\prime}\left(t_{k}\right) \Psi\left(1-\alpha_{k}\left(t_{k}\right)\right)}{\Gamma\left(2-\alpha_{k}\left(t_{k}\right)\right)} \int_{a_{k}}^{t_{k}}\left(t_{k}-\tau\right)^{1-\alpha_{k}\left(t_{k}\right)} \frac{\partial x}{\partial t_{k}}[\tau]_{k} d \tau .
\end{aligned}
$$

The rest of the proof is similar to the one of Theorem 32 .

Remark 34 As particular cases of Theorems [30, 32 and [33, we obtain expansion formulas for ${ }_{a}^{C} D_{t}^{\alpha(t)} x(t),{ }_{a}^{C} \mathcal{D}_{t}^{\alpha(t)} x(t)$ and ${ }_{a}^{C} \mathbb{D}_{t}^{\alpha(t)} x(t)$.

With respect to the three right fractional operators of Definition 31, we set, for $p \in \mathbb{N}$,

$$
\begin{aligned}
C_{p} & =\frac{(-1)^{p}}{\Gamma\left(p+1-\alpha_{k}\left(t_{k}\right)\right)}\left[1+\sum_{l=n-p+1}^{N} \frac{\Gamma\left(\alpha_{k}\left(t_{k}\right)-n+l\right)}{\Gamma\left(\alpha_{k}\left(t_{k}\right)-p\right)(l-n+p) !}\right], \\
D_{p} & =\frac{-\Gamma\left(\alpha_{k}\left(t_{k}\right)-n+p\right)}{\Gamma\left(1-\alpha_{k}\left(t_{k}\right)\right) \Gamma\left(\alpha_{k}\left(t_{k}\right)\right)(p-n) !}, \\
W_{p}(\bar{t}) & =\int_{t_{k}}^{b_{k}}\left(b_{k}-\tau\right)^{p-n} \frac{\partial x}{\partial t_{k}}[\tau]_{k} d \tau, \\
M_{p}(\bar{t}) & =\max _{\tau \in\left[t_{k}, b_{k}\right]}\left|\frac{\partial^{p} x}{\partial t_{k}^{p}}[\tau]_{k}\right| .
\end{aligned}
$$

The expansion formulas are given in Theorems 35,36 and 37. We omit the proofs since they are similar to the corresponding left ones.

Theorem 35 Let $x \in C^{n+1}\left(\prod_{i=1}^{m}\left[a_{i}, b_{i}\right], \mathbb{R}\right)$ with $n \in \mathbb{N}$. Then, for all $k \in$ $\{1, \ldots, m\}$ and for all $N \in \mathbb{N}$ such that $N \geq n$, we have

$$
\begin{aligned}
{ }_{t_{k}}^{C} \mathbb{D}_{b_{k}}^{\alpha_{k}\left(t_{k}\right)} x(\bar{t})= & \sum_{p=1}^{n} \\
& C_{p}\left(b_{k}-t_{k}\right)^{p-\alpha_{k}\left(t_{k}\right)} \frac{\partial^{p} x}{\partial t_{k}^{p}}\left[t_{k}\right]_{k} \\
& +\sum_{p=n}^{N} D_{p}\left(b_{k}-t_{k}\right)^{n-p-\alpha_{k}\left(t_{k}\right)} W_{p}(\bar{t})+E(\bar{t}) .
\end{aligned}
$$


The approximation error $E(\bar{t})$ is bounded by

$$
E(\bar{t}) \leq M_{n+1}(\bar{t}) \frac{\exp \left(\left(n-\alpha_{k}\left(t_{k}\right)\right)^{2}+n-\alpha_{k}\left(t_{k}\right)\right)}{\Gamma\left(n+1-\alpha_{k}\left(t_{k}\right)\right) N^{n-\alpha_{k}\left(t_{k}\right)}\left(n-\alpha_{k}\left(t_{k}\right)\right)}\left(b_{k}-t_{k}\right)^{n+1-\alpha_{k}\left(t_{k}\right)} .
$$

Theorem 36 Let $x \in C^{n+1}\left(\prod_{i=1}^{m}\left[a_{i}, b_{i}\right], \mathbb{R}\right)$ with $n \in \mathbb{N}$. Then, for all $k \in$ $\{1, \ldots, m\}$ and for all $N \in \mathbb{N}$ such that $N \geq n$, we have

$$
\begin{aligned}
& { }_{t_{k}}^{C} D_{b_{k}}^{\alpha_{k}\left(t_{k}\right)} x(\bar{t})=\sum_{p=1}^{n} C_{p}\left(b_{k}-t_{k}\right)^{p-\alpha_{k}\left(t_{k}\right)} \frac{\partial^{p} x}{\partial t_{k}^{p}}\left[t_{k}\right]_{k} \\
& +\sum_{p=n}^{N} D_{p}\left(b_{k}-t_{k}\right)^{n-p-\alpha_{k}\left(t_{k}\right)} W_{p}(\bar{t})+\frac{\alpha_{k}^{\prime}\left(t_{k}\right)\left(b_{k}-t_{k}\right)^{1-\alpha_{k}\left(t_{k}\right)}}{\Gamma\left(2-\alpha_{k}\left(t_{k}\right)\right)} \\
& \quad \times\left[\left(\frac{1}{1-\alpha_{k}\left(t_{k}\right)}-\ln \left(b_{k}-t_{k}\right)\right) \sum_{p=0}^{N}\left(\begin{array}{c}
1-\alpha_{k}\left(t_{k}\right) \\
p
\end{array}\right) \frac{(-1)^{p}}{\left(b_{k}-t_{k}\right)^{p}} W_{n+p}(\bar{t})\right. \\
& \left.+\sum_{p=0}^{N}\left(\begin{array}{c}
1-\alpha_{k}\left(t_{k}\right) \\
p
\end{array}\right)(-1)^{p} \sum_{r=1}^{N} \frac{1}{r\left(b_{k}-t_{k}\right)^{p+r}} W_{n+p+r}(\bar{t})\right]+E(\bar{t}) .
\end{aligned}
$$

The approximation error $E(\bar{t})$ is bounded by

$$
\begin{aligned}
E(\bar{t}) \leq & M_{n+1}(\bar{t}) \frac{\exp \left(\left(n-\alpha_{k}\left(t_{k}\right)\right)^{2}+n-\alpha_{k}\left(t_{k}\right)\right)}{\Gamma\left(n+1-\alpha_{k}\left(t_{k}\right)\right) N^{n-\alpha_{k}\left(t_{k}\right)}\left(n-\alpha_{k}\left(t_{k}\right)\right)}\left(b_{k}-t_{k}\right)^{n+1-\alpha_{k}\left(t_{k}\right)} \\
& +\left|\alpha_{k}^{\prime}\left(t_{k}\right)\right| M_{1}(\bar{t}) \frac{\exp \left(\left(1-\alpha_{k}\left(t_{k}\right)\right)^{2}+1-\alpha_{k}\left(t_{k}\right)\right)}{\Gamma\left(2-\alpha_{k}\left(t_{k}\right)\right) N^{1-\alpha_{k}\left(t_{k}\right)}\left(1-\alpha_{k}\left(t_{k}\right)\right)} \\
& \times\left[\left|\frac{1}{1-\alpha_{k}\left(t_{k}\right)}-\ln \left(b_{k}-t_{k}\right)\right|+\frac{1}{N}\right]\left(b_{k}-t_{k}\right)^{2-\alpha_{k}\left(t_{k}\right)} .
\end{aligned}
$$

Theorem 37 Let $x \in C^{n+1}\left(\prod_{i=1}^{m}\left[a_{i}, b_{i}\right], \mathbb{R}\right)$ with $n \in \mathbb{N}$. Then, for all $k \in$ $\{1, \ldots, m\}$ and for all $N \in \mathbb{N}$ such that $N \geq n$, we have

$$
\begin{aligned}
& { }_{t_{k}}^{C} D_{b_{k}}^{\alpha_{k}\left(t_{k}\right)} x(\bar{t})=\sum_{p=1}^{n} C_{p}\left(b_{k}-t_{k}\right)^{p-\alpha_{k}\left(t_{k}\right)} \frac{\partial^{p} x}{\partial t_{k}^{p}}\left[t_{k}\right]_{k} \\
& \quad+\sum_{p=n}^{N} D_{p}\left(b_{k}-t_{k}\right)^{n-p-\alpha_{k}\left(t_{k}\right)} W_{p}(\bar{t})+\frac{\alpha_{k}^{\prime}\left(t_{k}\right)\left(b_{k}-t_{k}\right)^{1-\alpha_{k}\left(t_{k}\right)}}{\Gamma\left(2-\alpha_{k}\left(t_{k}\right)\right)} \\
& \quad \times\left[\left(\Psi\left(2-\alpha_{k}\left(t_{k}\right)\right)-\ln \left(b_{k}-t_{k}\right)\right) \sum_{p=0}^{N}\left(\begin{array}{c}
1-\alpha_{k}\left(t_{k}\right) \\
p
\end{array}\right) \frac{(-1)^{p}}{\left(b_{k}-t_{k}\right)^{p}} W_{n+p}(\bar{t})\right. \\
& \left.\quad+\sum_{p=0}^{N}\left(\begin{array}{c}
1-\alpha_{k}\left(t_{k}\right) \\
p
\end{array}\right)(-1)^{p} \sum_{r=1}^{N} \frac{1}{r\left(b_{k}-t_{k}\right)^{p+r}} W_{n+p+r}(\bar{t})\right]+E(\bar{t}) .
\end{aligned}
$$

The approximation error $E(\bar{t})$ is bounded by 


$$
\begin{aligned}
E(\bar{t}) \leq & M_{n+1}(\bar{t}) \frac{\exp \left(\left(n-\alpha_{k}\left(t_{k}\right)\right)^{2}+n-\alpha_{k}\left(t_{k}\right)\right)}{\Gamma\left(n+1-\alpha_{k}\left(t_{k}\right)\right) N^{n-\alpha_{k}\left(t_{k}\right)}\left(n-\alpha_{k}\left(t_{k}\right)\right)}\left(b_{k}-t_{k}\right)^{n+1-\alpha_{k}\left(t_{k}\right)} \\
& +\left|\alpha_{k}^{\prime}\left(t_{k}\right)\right| M_{1}(\bar{t}) \frac{\exp \left(\left(1-\alpha_{k}\left(t_{k}\right)\right)^{2}+1-\alpha_{k}\left(t_{k}\right)\right)}{\Gamma\left(2-\alpha_{k}\left(t_{k}\right)\right) N^{1-\alpha_{k}\left(t_{k}\right)}\left(1-\alpha_{k}\left(t_{k}\right)\right)} \\
& \times\left[\left|\Psi\left(2-\alpha_{k}\left(t_{k}\right)\right)-\ln \left(b_{k}-t_{k}\right)\right|+\frac{1}{N}\right]\left(b_{k}-t_{k}\right)^{2-\alpha_{k}\left(t_{k}\right)} .
\end{aligned}
$$

\subsection{Example}

To test the accuracy of the proposed method, we compare the fractional derivative of a concrete given function with some numerical approximations of it. For $t \in[0,1]$, let $x(t)=t^{2}$ be the test function. For the order of the fractional derivatives we consider two cases:

$$
\alpha(t)=\frac{50 t+49}{100} \text { and } \beta(t)=\frac{t+5}{10} .
$$

We consider the approximations given in Theorems 30, 32 and 33, with a fixed $n=1$ and $N \in\{2,4,6\}$. The error of approximating $f(t)$ by $\tilde{f}(t)$ is measured by $|f(t)-\tilde{f}(t)|$. See Figures 3.23 .7

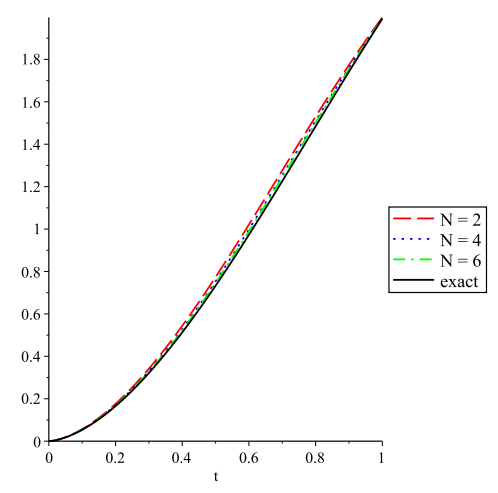

(a) ${ }_{0}^{C} \mathbb{D}_{t}^{\alpha(t)} x(t)$

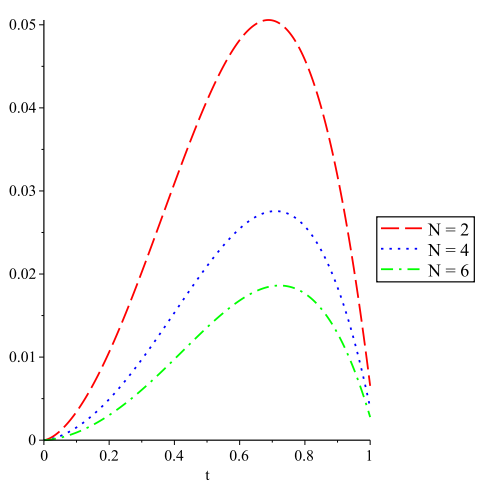

(b) Error

Fig. 3.2. Type III left Caputo derivative of order $\alpha(t)$ for the example of Section 3.3 - analytic versus numerical approximations obtained from Theorem 30.

\subsection{Applications}

In this section we apply the proposed technique to some concrete fractional differential equations of physical relevance. 


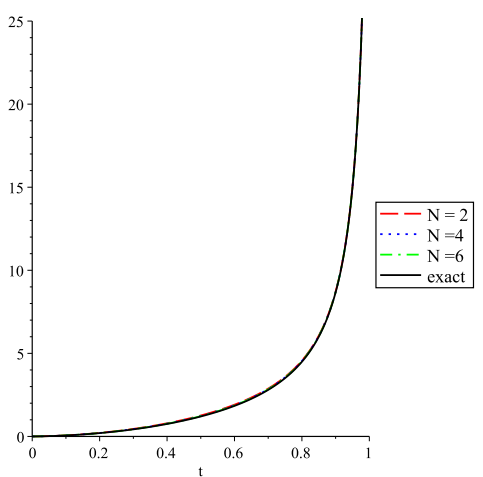

(a) ${ }_{0}^{C} D_{t}^{\alpha(t)} x(t)$

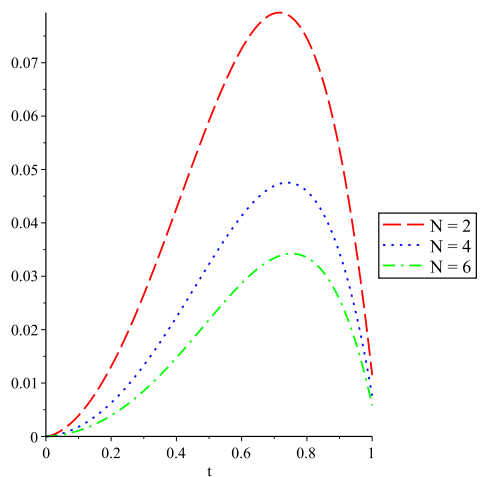

(b) Error

Fig. 3.3. Type I left Caputo derivative of order $\alpha(t)$ for the example of Section 3.3 analytic versus numerical approximations obtained from Theorem 32

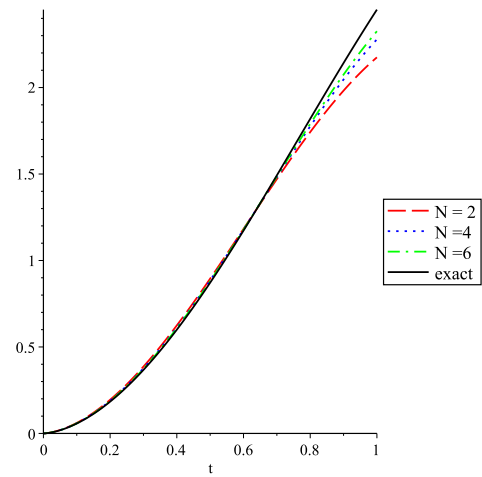

(a) ${ }_{0}^{C} \mathcal{D}_{t}^{\alpha(t)} x(t)$

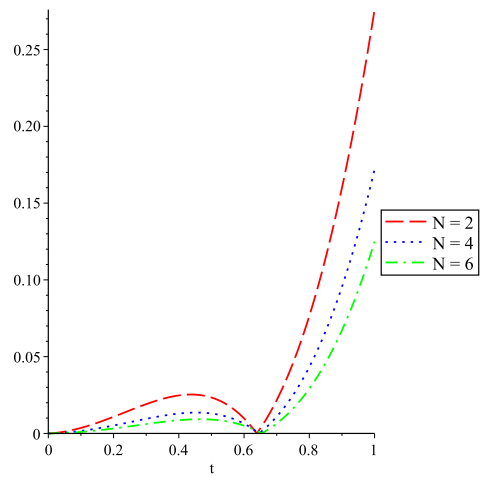

(b) Error

Fig. 3.4. Type II left Caputo derivative of order $\alpha(t)$ for the example of Section 3.3 analytic versus numerical approximations obtained from Theorem 33

\subsubsection{A time-fractional diffusion equation}

We extend the one-dimensional time-fractional diffusion equation ( $\mathrm{Lin}$ and $\mathrm{Xu}$, 2007) to the variable-order case. Consider $u=u(x, t)$ with domain $[0,1]^{2}$. The partial fractional differential equation of order $\alpha(t)$ is the following:

$$
{ }_{0}^{C} \mathbb{D}_{t}^{\alpha(t)} u(x, t)-\frac{\partial^{2} u}{\partial x^{2}}(x, t)=f(x, t) \quad \text { for } x \in[0,1], \quad t \in[0,1],
$$

subject to the boundary conditions

$$
u(x, 0)=g(x), \quad \text { for } x \in(0,1),
$$




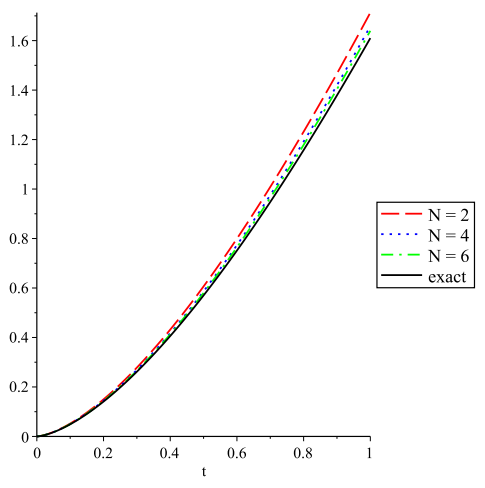

(a) ${ }_{0}^{C} \mathbb{D}_{t}^{\beta(t)} x(t)$

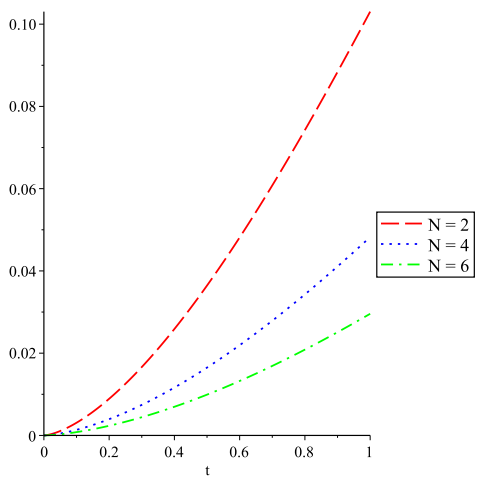

(b) Error

Fig. 3.5. Type III left Caputo derivative of order $\beta(t)$ for the example of Section 3.3 - analytic versus numerical approximations obtained from Theorem 30.

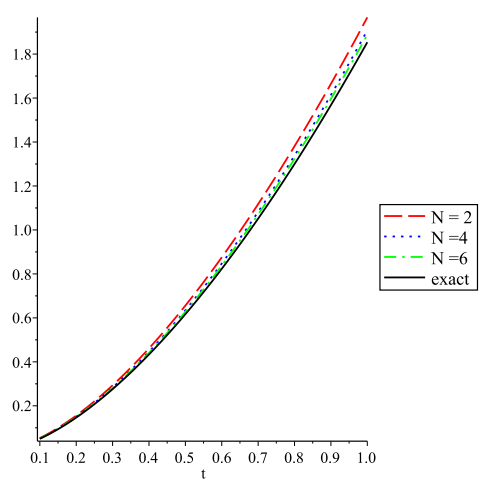

(a) ${ }_{0}^{C} D_{t}^{\beta(t)} x(t)$

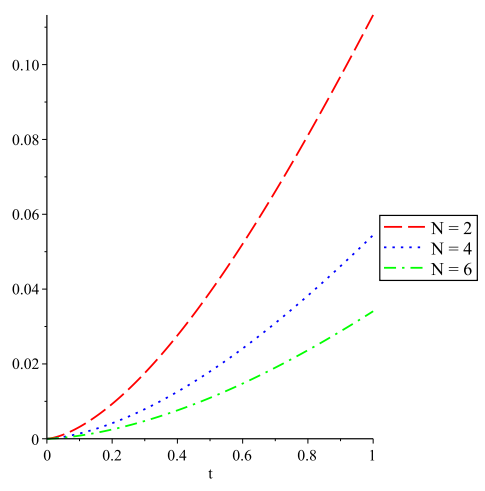

(b) Error

Fig. 3.6. Type I left Caputo derivative of order $\beta(t)$ for the example of Section 3.3 analytic versus numerical approximations obtained from Theorem 32

and

$$
u(0, t)=u(1, t)=0, \quad \text { for } t \in[0,1] .
$$

We mention that when $\alpha(t) \equiv 1$, one obtains the classical diffusion equation, and when $\alpha(t) \equiv 0$ one gets the classical Helmholtz elliptic equation. Using Lemma 27, it is easy to check that

$$
u(x, t)=t^{2} \sin (2 \pi x)
$$

is a solution to (3.8)-(3.10) with 


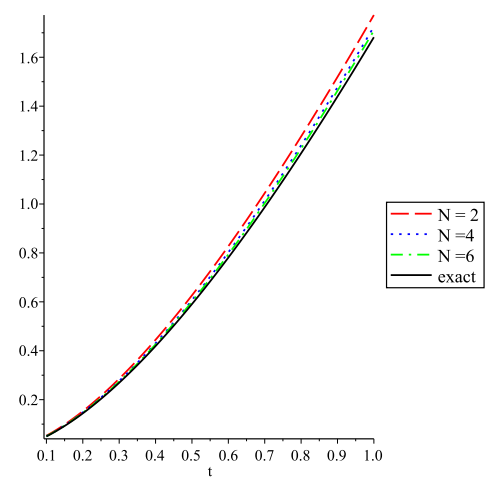

(a) ${ }_{0}^{C} \mathcal{D}_{t}^{\beta(t)} x(t)$

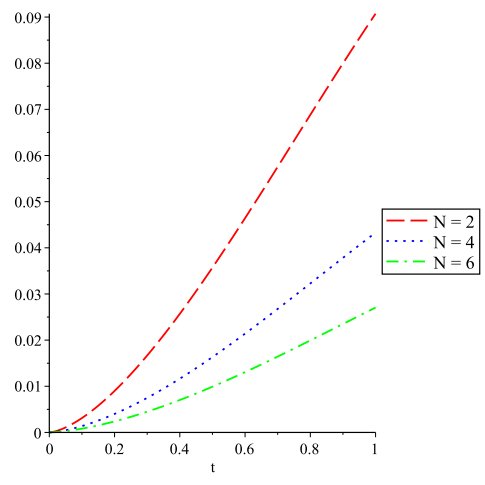

(b) Error

Fig. 3.7. Type II left Caputo derivative of order $\beta(t)$ for the example of Section 3.3 analytic versus numerical approximations obtained from Theorem 33

$$
f(x, t)=\left(\frac{2}{\Gamma(3-\alpha(t))} t^{2-\alpha(t)}+4 \pi^{2} t^{2}\right) \sin (2 \pi x)
$$

and

$$
g(x)=0
$$

(compare with Example 1 in (Lin and $\mathrm{Xu}, 2007)$ ). The numerical procedure is the following: replace ${ }_{0}^{C} \mathbb{D}_{t}^{\alpha(t)} u$ with the approximation given in Theorem 30 . taking $n=1$ and an arbitrary $N \geq 1$, that is,

$$
{ }_{0}^{C} \mathbb{D}_{t}^{\alpha(t)} u(x, t) \approx A t^{1-\alpha(t)} \frac{\partial u}{\partial t}(x, t)+\sum_{p=1}^{N} B_{p} t^{1-p-\alpha(t)} V_{p}(x, t)
$$

with

$$
\begin{aligned}
A & =\frac{1}{\Gamma(2-\alpha(t))}\left[1+\sum_{l=1}^{N} \frac{\Gamma(\alpha(t)-1+l)}{\Gamma(\alpha(t)-1) l !}\right], \\
B_{p} & =\frac{\Gamma(\alpha(t)-1+p)}{\Gamma(1-\alpha(t)) \Gamma(\alpha(t))(p-1) !}, \\
V_{p}(x, t) & =\int_{0}^{t} \tau^{p-1} \frac{\partial u}{\partial t}(x, \tau) d \tau .
\end{aligned}
$$

Then, the initial fractional problem (3.8)-(3.10) is approximated by the following system of second-order partial differential equations:

$$
A t^{1-\alpha(t)} \frac{\partial u}{\partial t}(x, t)+\sum_{p=1}^{N} B_{p} t^{1-p-\alpha(t)} V_{p}(x, t)-\frac{\partial^{2} u}{\partial x^{2}}(x, t)=f(x, t)
$$


and

$$
\frac{\partial V_{p}}{\partial t}(x, t)=t^{p-1} \frac{\partial u}{\partial t}(x, t), \quad p=1, \ldots, N,
$$

for $x \in[0,1]$ and for $t \in[0,1]$, subject to the boundary conditions

$$
\begin{gathered}
u(x, 0)=0, \quad \text { for } x \in(0,1), \\
u(0, t)=u(1, t)=0, \quad \text { for } t \in[0,1],
\end{gathered}
$$

and

$$
V_{p}(x, 0)=0, \quad \text { for } x \in[0,1], \quad p=1, \ldots, N \text {. }
$$

\subsubsection{A fractional partial differential equation in fluid mechanics}

We now apply our approximation techniques to the following one-dimensional linear inhomogeneous fractional Burgers' equation of variable-order (see (Odibat and Momani, 2009, Example 5.2)):

$$
{ }_{0}^{C} \mathbb{D}_{t}^{\alpha(t)} u(x, t)+\frac{\partial u}{\partial x}(x, t)-\frac{\partial^{2} u}{\partial x^{2}}(x, t)=\frac{2 t^{2-\alpha(t)}}{\Gamma(3-\alpha(t))}+2 x-2,
$$

for $x \in[0,1]$ and $t \in[0,1]$, subject to the boundary condition

$$
u(x, 0)=x^{2}, \quad \text { for } x \in(0,1) .
$$

Here,

$$
F(x, t)=\frac{2 t^{2-\alpha(t)}}{\Gamma(3-\alpha(t))}+2 x-2
$$

is the external force field. Burgers' equation is used to model gas dynamics, traffic flow, turbulence, fluid mechanics, etc. The exact solution is

$$
u(x, t)=x^{2}+t^{2} .
$$

The fractional problem (3.11)-3.12) can be approximated by

$$
\begin{aligned}
A t^{1-\alpha(t)} \frac{\partial u}{\partial t}(x, t)+\sum_{p=1}^{N} B_{p} t^{1-p-\alpha(t)} V_{p}(x, t)+\frac{\partial u}{\partial x} & (x, t)-\frac{\partial^{2} u}{\partial x^{2}}(x, t) \\
& =\frac{2 t^{2-\alpha(t)}}{\Gamma(3-\alpha(t))}+2 x-2
\end{aligned}
$$

with $A, B_{p}$ and $V_{p}, p \in\{1, \ldots, N\}$, as in Section 3.4.1 The approximation error can be decreased as much as desired by increasing the value of $N$. 


\section{References}

Caputo M (1967) Linear model of dissipation whose $Q$ is almost frequency independent-II. Geophys. J. R. Astr. Soc. 13:529-539

Chechkin AV, Gorenflo R, Sokolov IM (2005) Fractional diffusion in inhomogeneous media. J. Phys. A: Math. Theor. 38(42):L679-L684

Dalir M, Bashour M (2010) Applications of fractional calculus. Appl. Math. Sci. 4(21-24):1021-1032

Diethelm K (2004) The Analysis of Fractional Differential Equations. Lecture Notes in Mathematics 2004, Springer, Berlin

Džrbašjan MM, Nersesjan AB (1968) Fractional derivatives and the Cauchy problem for differential equations of fractional order. Izv. Akad. Nauk Armjan. SSR Ser. Mat. 3(1):3-29

Lin Y, Xu C (2007) Finite difference/spectral approximations for the timefractional diffusion equation. J. Comput. Phys. 225(2):1533-1552

Machado JAT, Silva MF, Barbosa RS, Jesus IS, Reis CM, Marcos MG, Galhano AF (2010) Some applications of fractional calculus in engineering. Math. Probl. Eng. 2010:Art. ID 639801, 34 pp

Murio DA, Mejía CE (2008) Generalized time fractional IHCP with Caputo fractional derivatives. J. Phys. Conf. Ser. 135:Art. ID 012074, 8 pp

Odibat Z, Momani S (2009) The variational iteration method: an efficient scheme for handling fractional partial differential equations in fluid mechanics. Comput. Math. Appl. 58(11-12):2199-2208

Rabotnov YN (1969) Creep Problems in Structural Members. North-Holland Series in Applied Mathematics and Mechanics, Amsterdam/London

Samko SG, Kilbas AA, Marichev OI (1993) Fractional Integrals and Derivatives. translated from the 1987 Russian original, Gordon and Breach, Yverdon

Samko SG, Ross B (1993) Integration and differentiation to a variable fractional order. Integral Transform. Spec. Funct. 1(4):277-300

Santamaria F, Wils S, Schutter E, Augustine GJ (2006) Anomalous diffusion in Purkinje cell dendrites caused by spines. Neuron. 52:635-648

Singh K, Saxena R, Kumar S (2013) Caputo-based fractional derivative in fractional Fourier transform domain. IEEE Journal on Emerging and Selected Topics in Circuits and Systems 3:330-337

Sun HG, Chen W, Chen YQ (2009) Variable order fractional differential operators in anomalous diffusion modeling. Physica A. 388(21):4586-4592

Sweilam NH, AL-Mrawm HM (2011) On the numerical solutions of the variable order fractional heat equation. Studies in Nonlinear Sciences 2:31-36

Tavares D, Almeida R, Torres DFM (2016) Caputo derivatives of fractional variable order: numerical approximations. Commun. Nonlinear Sci. Numer. Simul. 35:69-87 arXiv: 1511.02017

Yajima T, Yamasaki K (2012) Geometry of surfaces with Caputo fractional derivatives and applications to incompressible two-dimensional flows. J. Phys. A 45(6):065201, 15 pp 



\section{The fractional calculus of variations}

In this chapter, we consider general fractional problems of the calculus of variations, where the Lagrangian depends on a combined Caputo fractional derivative of variable fractional order ${ }^{C} D_{\gamma}^{\alpha(\cdot, \cdot), \beta(\cdot, \cdot)}$ given as a combination of the left and the right Caputo fractional derivatives of orders, respectively, $\alpha(\cdot, \cdot)$ and $\beta(\cdot, \cdot)$. More specifically, here we study some problems of the calculus of variations with integrands depending on the independent variable $t$, an arbitrary function $x$ and a fractional derivative ${ }^{C} D_{\gamma}^{\alpha(\cdot, \cdot), \beta(\cdot, \cdot)} x$ defined by

$$
{ }^{C} D_{\gamma}^{\alpha(\cdot, \cdot), \beta(\cdot, \cdot)} x(t)=\gamma_{1}{ }_{a}^{C} D_{t}^{\alpha(\cdot, \cdot)} x(t)+\gamma_{2}{ }_{t}^{C} D_{b}^{\beta(\cdot, \cdot)} x(t),
$$

where $\gamma=\left(\gamma_{1}, \gamma_{2}\right) \in[0,1]^{2}$, with $\gamma_{1}$ and $\gamma_{2}$ not both zero.

Starting from the fundamental variational problem, we investigate different types of variational problems: problems with time delay or with higher-order derivatives, isoperimetric problems, problems with holonomic constraints and problems of Herglotz and those depending on combined Caputo fractional derivatives of variable-order. Our variational problems are known as free-time problems because, in general, we impose a boundary condition at the initial time $t=a$, but we consider the endpoint $b$ of the integral and the terminal state $x(b)$ to be free (variable). The main results provide necessary optimality conditions of Euler-Lagrange type, described by fractional differential equations of variable-order, and different transversality optimality conditions.

Our main contributions are to consider the Lagrangian containing a fractional operator where the order is not a constant, and may depend on time. Moreover, we do not only assume that $x(b)$ is free, but the endpoint $b$ is also free.

In Section 4.1, we introduce the combined Caputo fractional derivative of variable-order and provide the necessary concepts and results needed in the sequel (Section 4.1.1). We deduce two formulas of integration by parts that involve the combined Caputo fractional derivative of higher order (Section 4.1.2). 
The fractional variational problems under our consideration are formulated in terms of the fractional derivative ${ }^{C} D_{\gamma}^{\alpha(\cdot, \cdot), \beta(\cdot, \cdot)}$. We discuss the fundamental concepts of a variational calculus such as the Euler-Lagrange equations and transversality conditions (Section 4.2), variational problems involving higher-order derivatives (Section 4.3), variational problems with time delay (Section 4.4), isoperimetric problems (Section 4.5), problems with holonomic constraints (Section 4.6) and the last Section 4.7 investigates fractional variational Herglotz problems.

Some illustrative examples are presented for all considered variational problems.

The results of this chapter first appeared in (Tavares, Almeida and Torres, 2015, 2017, 2018a, b).

\subsection{Introduction}

In this section, we recall the fundamental definition of the combined Caputo fractional derivative presented in Section 1.3.3 (see Definition 15), and generalize it for fractional variable-order. In the end, we prove an integration by parts formula, involving the higher-order Caputo fractional derivative of variable-order.

\subsubsection{Combined operators of variable-order}

Motivated by the combined fractional Caputo derivative, we propose the following definitions about combined variable-order fractional calculus.

Let $\alpha, \beta:[a, b]^{2} \rightarrow(0,1)$ be two functions and $\gamma=\left(\gamma_{1}, \gamma_{2}\right) \in[0,1]^{2}$ a vector, with $\gamma_{1}$ and $\gamma_{2}$ not both zero.

Definition 32 The combined Riemann-Liouville fractional derivative of variableorder, denoted by $D_{\gamma}^{\alpha(\cdot, \cdot), \beta(\cdot, \cdot)}$, is defined by

$$
D_{\gamma}^{\alpha(\cdot, \cdot), \beta(\cdot, \cdot)}=\gamma_{1 a} D_{t}^{\alpha(\cdot, \cdot)}+\gamma_{2 t} D_{b}^{\beta(\cdot, \cdot)},
$$

acting on $x \in C([a, b] ; \mathbb{R})$ in the following way:

$$
D_{\gamma}^{\alpha(\cdot, \cdot), \beta(\cdot, \cdot)} x(t)=\gamma_{1 a} D_{t}^{\alpha(\cdot, \cdot)} x(t)+\gamma_{2} D_{b}^{\beta(\cdot, \cdot)} x(t) .
$$

Definition 33 The combined Caputo fractional derivative operator of variableorder, denoted by ${ }^{C} D_{\gamma}^{\alpha(\cdot, \cdot), \beta(\cdot, \cdot)}$, is defined by

$$
{ }^{C} D_{\gamma}^{\alpha(\cdot, \cdot), \beta(\cdot, \cdot)}=\gamma_{1}{ }_{a}^{C} D_{t}^{\alpha(\cdot, \cdot)}+\gamma_{2}{ }_{t}^{C} D_{b}^{\beta(\cdot, \cdot)},
$$

acting on $x \in C^{1}([a, b] ; \mathbb{R})$ in the following way:

$$
{ }^{C} D_{\gamma}^{\alpha(\cdot, \cdot), \beta(\cdot, \cdot)} x(t)=\gamma_{1}{ }_{a}^{C} D_{t}^{\alpha(\cdot, \cdot)} x(t)+\gamma_{2}{ }_{t}^{C} D_{b}^{\beta(\cdot, \cdot)} x(t) .
$$


In the sequel, we need the auxiliary notation of the dual fractional derivative, defined by

$$
D_{\bar{\gamma}}^{\beta(\cdot, \cdot), \alpha(\cdot, \cdot)}=\gamma_{2 a} D_{t}^{\beta(\cdot, \cdot)}+\gamma_{1 t} D_{T}^{\alpha(\cdot, \cdot)},
$$

where $\bar{\gamma}=\left(\gamma_{2}, \gamma_{1}\right)$ and $T \in(a, b]$.

To generalize the concept of the combined fractional derivative to higher orders, we need to review some definitions of higher-order operators.

Let $n \in \mathbb{N}$ and $x:[a, b] \rightarrow \mathbb{R}$ be a function of class $C^{n}$. The fractional order is a continuous function of two variables, $\alpha_{n}:[a, b]^{2} \rightarrow(n-1, n)$.

Definition 34 The left and right Riemann-Liouville fractional integrals of order $\alpha_{n}(\cdot, \cdot)$ are defined respectively by

$$
{ }_{a} I_{t}^{\alpha_{n}(\cdot, \cdot)} x(t)=\int_{a}^{t} \frac{1}{\Gamma\left(\alpha_{n}(t, \tau)\right)}(t-\tau)^{\alpha_{n}(t, \tau)-1} x(\tau) d \tau
$$

and

$$
{ }_{t} I_{b}^{\alpha_{n}(\cdot, \cdot)} x(t)=\int_{t}^{b} \frac{1}{\Gamma\left(\alpha_{n}(\tau, t)\right)}(\tau-t)^{\alpha_{n}(\tau, t)-1} x(\tau) d \tau .
$$

With this definition of Riemann-Liouville fractional integrals of variableorder, and considering $n=1$, in Appendix A.1 we implemented two functions leftFI ( $x, a l p h a, a)$ and $\operatorname{rightFI}(x, a l p h a, b)$ that approximate, respectively, the Riemann-Liouville fractional integrals ${ }_{a} I_{+}^{\alpha_{n}(\cdot, \cdot)} x$ and ${ }_{t} I_{b}^{\alpha_{n}(\cdot, \cdot)} x$, using the open source software package Chebfun (Trefethen, 2013). With these two functions we present also, in Appendix A.1, an illustrative example where we determine computational approximations to the fractional integrals for a specific power function of the form $x(t)=t^{\gamma}$ (see Example 4.4 in Appendix A.1). For this numerical computations, we have used MATLAB (Linge and Langtangen, 2016).

Definition 35 The left and right Riemann-Liouville fractional derivatives of order $\alpha_{n}(\cdot, \cdot)$ are defined by

$$
{ }_{a} D_{t}^{\alpha_{n}(\cdot, \cdot)} x(t)=\frac{d^{n}}{d t^{n}} \int_{a}^{t} \frac{1}{\Gamma\left(n-\alpha_{n}(t, \tau)\right)}(t-\tau)^{n-1-\alpha_{n}(t, \tau)} x(\tau) d \tau
$$

and

$$
{ }_{t} D_{b}^{\alpha_{n}(\cdot, \cdot)} x(t)=(-1)^{n} \frac{d^{n}}{d t^{n}} \int_{t}^{b} \frac{1}{\Gamma\left(n-\alpha_{n}(\tau, t)\right)}(\tau-t)^{n-1-\alpha_{n}(\tau, t)} x(\tau) d \tau,
$$

respectively.

Definition 36 The left and right Caputo fractional derivatives of order $\alpha_{n}(\cdot, \cdot)$ are defined by 


$$
{ }_{a}^{C} D_{t}^{\alpha_{n}(\cdot, \cdot)} x(t)=\int_{a}^{t} \frac{1}{\Gamma\left(n-\alpha_{n}(t, \tau)\right)}(t-\tau)^{n-1-\alpha_{n}(t, \tau)} x^{(n)}(\tau) d \tau
$$

and

$$
{ }_{t}^{C} D_{b}^{\alpha_{n}(\cdot, \cdot)} x(t)=(-1)^{n} \int_{t}^{b} \frac{1}{\Gamma\left(n-\alpha_{n}(\tau, t)\right)}(\tau-t)^{n-1-\alpha_{n}(\tau, t)} x^{(n)}(\tau) d \tau,
$$

respectively.

Remark 38 Definitions 35] and 36, for the particular case of order between 0 and 1, can be found in (Malinowska. Odzijewicz and Torres, 2015). They seem to be new for the higher-order case.

Considering Definition 36, in Appendix A.2 we implement two new functions leftCaputo (x,alpha, a, n) and rightCaputo $(\mathrm{x}, \mathrm{alpha}, \mathrm{b}, \mathrm{n})$ that approximate the higher-order Caputo fractional derivatives ${ }_{a}^{C} D_{t}^{\alpha_{n}(\cdot, \cdot)} x$ and ${ }_{t}^{C} D_{b}^{\alpha_{n}(\cdot, \cdot)} x$, respectively. With these two functions, we present, also in Appendix A.2, an illustrative example where we study approximations to the Caputo fractional derivatives for a specific power function of the form $x(t)=t^{\gamma}$ (see Example 4.5 in Appendix A.2).

Remark 39 From Definition 34, it follows that

$$
{ }_{a} D_{t}^{\alpha_{n}(\cdot, \cdot)} x(t)=\frac{d^{n}}{d t^{n}}{ }_{a} I_{t}^{n-\alpha_{n}(\cdot, \cdot)} x(t),{ }_{t} D_{b}^{\alpha_{n}(\cdot, \cdot)} x(t)=(-1)^{n} \frac{d^{n}}{d t^{n}}{ }_{t} I_{b}^{n-\alpha_{n}(\cdot, \cdot)} x(t)
$$

and

${ }_{a}^{C} D_{t}^{\alpha_{n}(\cdot, \cdot)} x(t)={ }_{a} I_{t}^{n-\alpha_{n}(\cdot, \cdot)} \frac{d^{n}}{d t^{n}} x(t),{ }_{t}^{C} D_{b}^{\alpha_{n}(\cdot, \cdot)} x(t)=(-1)^{n}{ }_{t} I_{b}^{n-\alpha_{n}(\cdot, \cdot)} \frac{d^{n}}{d t^{n}} x(t)$.

In Lemma 40, we obtain higher-order Caputo fractional derivatives of a power function. For that, we assume that the fractional order depends only on the first variable: $\alpha_{n}(t, \tau):=\bar{\alpha}_{n}(t)$, where $\bar{\alpha}_{n}:[a, b] \rightarrow(n-1, n)$ is a given function.

Lemma 40 Let $x(t)=(t-a)^{\gamma}$ with $\gamma>n-1$. Then,

$$
{ }_{a}^{C} D_{t}^{\bar{\alpha}_{n}(t)} x(t)=\frac{\Gamma(\gamma+1)}{\Gamma\left(\gamma-\bar{\alpha}_{n}(t)+1\right)}(t-a)^{\gamma-\bar{\alpha}_{n}(t)} .
$$

Proof. As $x(t)=(t-a)^{\gamma}$, if we differentiate it $n$ times, we obtain

$$
x^{(n)}(t)=\frac{\Gamma(\gamma+1)}{\Gamma(\gamma-n+1)}(t-a)^{\gamma-n} .
$$

Using Definition 36 of the left Caputo fractional derivative, we get 


$$
\begin{aligned}
{ }_{a}^{C} D_{t}^{\bar{\alpha}_{n}(t)} x(t) & =\int_{a}^{t} \frac{1}{\Gamma\left(n-\bar{\alpha}_{n}(t)\right)}(t-\tau)^{n-1-\bar{\alpha}_{n}(t)} x^{(n)}(\tau) d \tau \\
& =\int_{a}^{t} \frac{\Gamma(\gamma+1)}{\Gamma(\gamma-n+1) \Gamma\left(n-\bar{\alpha}_{n}(t)\right)}(t-\tau)^{n-1-\bar{\alpha}_{n}(t)}(\tau-a)^{\gamma-n} d \tau .
\end{aligned}
$$

Now, we proceed with the change of variables $\tau-a=s(t-a)$. Using the Beta function $B(\cdot, \cdot)$, we obtain that

$$
\begin{aligned}
{ }_{a}^{C} D_{t}^{\bar{\alpha}_{n}(t)} x(t)= & \frac{\Gamma(\gamma+1)}{\Gamma(\gamma-n+1) \Gamma\left(n-\bar{\alpha}_{n}(t)\right)} \\
& \times \int_{0}^{1}(1-s)^{n-1-\bar{\alpha}_{n}(t)} s^{\gamma-n}(t-a)^{\gamma-\bar{\alpha}_{n}(t)} d s \\
= & \frac{\Gamma(\gamma+1)(t-a)^{\gamma-\bar{\alpha}_{n}(t)}}{\Gamma(\gamma-n+1) \Gamma\left(n-\bar{\alpha}_{n}(t)\right)} B\left(\gamma-n+1, n-\bar{\alpha}_{n}(t)\right) \\
= & \frac{\Gamma(\gamma+1)}{\Gamma\left(\gamma-\bar{\alpha}_{n}(t)+1\right)} \quad(t-a)^{\gamma-\bar{\alpha}_{n}(t)} .
\end{aligned}
$$

The proof is complete.

Considering the higher-order left Caputo fractional derivative's formula of a power function of the form $x(t)=(t-a)^{\gamma}$, deduced before, in Appendix A.2 we determine the left Caputo fractional derivative of the particular function $x(t)=t^{4}$ for several values of $t$ and compare them with the approximated values obtained by the Chebfun function leftCaputo ( $x$, alpha, $a, n$ ) (see Example 4.7 in Appendix A.2).

For our next result, we assume that the fractional order depends only on the second variable: $\alpha_{n}(\tau, t):=\bar{\alpha}_{n}(t)$, where $\bar{\alpha}_{n}:[a, b] \rightarrow(n-1, n)$ is a given function. The proof is similar to that of Lemma 40, and so we omit it here.

Lemma 41 Let $x(t)=(b-t)^{\gamma}$ with $\gamma>n-1$. Then,

$$
{ }_{t}^{C} D_{b}^{\bar{\alpha}_{n}(t)} x(t)=\frac{\Gamma(\gamma+1)}{\Gamma\left(\gamma-\bar{\alpha}_{n}(t)+1\right)}(b-t)^{\gamma-\bar{\alpha}_{n}(t)} .
$$

The next step is to consider a linear combination of the previous fractional derivatives to define the combined fractional operators for higher-order.

Let $\alpha_{n}, \beta_{n}:[a, b]^{2} \rightarrow(n-1, n)$ be two variable fractional orders, $\gamma^{n}=$ $\left(\gamma_{1}^{n}, \gamma_{2}^{n}\right) \in[0,1]^{2}$ a vector, with $\gamma_{1}$ and $\gamma_{2}$ not both zero, and $x \in C^{n}([a, b] ; \mathbb{R})$ a function.

Definition 37 The higher-order combined Riemann-Liouville fractional derivative is defined by

$$
D_{\gamma^{n}}^{\alpha_{n}(\cdot, \cdot), \beta_{n}(\cdot, \cdot)}=\gamma_{1 a}^{n} D_{t}^{\alpha_{n}(\cdot, \cdot)}+\gamma_{2 t}^{n} D_{b}^{\beta_{n}(\cdot, \cdot)},
$$

acting on $x \in C^{n}([a, b] ; \mathbb{R})$ in the following way:

$$
D_{\gamma^{n}}^{\alpha_{n}(\cdot, \cdot), \beta_{n}(\cdot, \cdot)} x(t)=\gamma_{1}^{n}{ }_{a} D_{t}^{\alpha_{n}(\cdot, \cdot)} x(t)+\gamma_{2}^{n}{ }_{t} D_{b}^{\beta_{n}(\cdot, \cdot)} x(t) .
$$


In our work, we use both Riemann-Liouville and Caputo derivatives definitions. The emphasis is, however, in Caputo fractional derivatives.

Definition 38 The higher-order combined Caputo fractional derivative of $x$ at $t$ is defined by

$$
{ }^{C} D_{\gamma^{n}}^{\alpha_{n}(\cdot, \cdot), \beta_{n}(\cdot, \cdot)} x(t)=\gamma_{1}^{n}{ }_{a}^{C} D_{t}^{\alpha_{n}(\cdot, \cdot)} x(t)+\gamma_{2}^{n}{ }_{t}^{C} D_{b}^{\beta_{n}(\cdot, \cdot)} x(t) .
$$

Similarly, in the sequel of this work, we need the auxiliary notation of the dual fractional derivative:

$$
D \frac{\beta_{i}(\cdot, \cdot), \alpha_{i}(\cdot, \cdot)}{\gamma^{i}}=\gamma_{2}^{i}{ }_{a} D_{t}^{\beta_{i}(\cdot, \cdot)}+\gamma_{1}^{i}{ }_{t} D_{T}^{\alpha_{i}(\cdot, \cdot)},
$$

where $\overline{\gamma^{i}}=\left(\gamma_{2}^{i}, \gamma_{1}^{i}\right)$ and $T \in(a, b]$.

Some computational aspects about the combined Caputo fractional derivative of variable-order, are also discussed in Appendix A.3, using the software package Chebfun. For that, we developed the new function (see Example 4.8 in Appendix A.3) combinedCaputo ( $\mathrm{x}$, alpha, beta, gamma1, gamma2, a, b, n) and obtained approximated values for a particular power function.

\subsubsection{Generalized fractional integration by parts}

When dealing with variational problems, one key property is integration by parts. Formulas of integration by parts have an important role in the proof of Euler-Lagrange conditions. In the following theorem, such formulas are proved for integrals involving higher-order Caputo fractional derivatives of variable-order.

Let $n \in \mathbb{N}$ and $x, y \in C^{n}([a, b] ; \mathbb{R})$ be two functions. The fractional order is a continuous function of two variables, $\alpha_{n}:[a, b]^{2} \rightarrow(n-1, n)$.

Theorem 42 The higher-order Caputo fractional derivatives of variableorder satisfy the integration by parts formulas

$$
\begin{aligned}
\int_{a}^{b} y(t){ }_{a}^{C} D_{t}^{\alpha_{n}(\cdot, \cdot)} x(t) d t & =\int_{a}^{b} x(t){ }_{t} D_{b}^{\alpha_{n}(\cdot, \cdot)} y(t) d t \\
& +\left[\sum_{k=0}^{n-1}(-1)^{k} x^{(n-1-k)}(t) \frac{d^{k}}{d t^{k}} I_{b}^{n-\alpha_{n}(\cdot, \cdot)} y(t)\right]_{t=a}^{t=b}
\end{aligned}
$$

and

$$
\begin{aligned}
\int_{a}^{b} y(t){ }_{t}^{C} D_{b}^{\alpha_{n}(\cdot, \cdot)} x(t) d t & =\int_{a}^{b} x(t){ }_{a} D_{t}^{\alpha_{n}(\cdot, \cdot)} y(t) d t \\
& +\left[\sum_{k=0}^{n-1}(-1)^{n+k} x^{(n-1-k)}(t) \frac{d^{k}}{d t^{k}} I_{t}^{n-\alpha_{n}(\cdot, \cdot)} y(t)\right]_{t=a}^{t=b} .
\end{aligned}
$$


Proof. Considering the Definition [36] of left Caputo fractional derivatives of order $\alpha_{n}(\cdot, \cdot)$, we obtain

$$
\begin{aligned}
\int_{a}^{b} y(t){ }_{a}^{C} D_{t}^{\alpha_{n}(\cdot, \cdot)} & x(t) d t \\
& =\int_{a}^{b} \int_{a}^{t} y(t) \frac{1}{\Gamma\left(n-\alpha_{n}(t, \tau)\right)}(t-\tau)^{n-1-\alpha_{n}(t, \tau)} x^{(n)}(\tau) d \tau d t
\end{aligned}
$$

Using Dirichelt's Formula, we rewrite it as

$$
\begin{aligned}
\int_{a}^{b} & \int_{t}^{b} y(\tau) \frac{(\tau-t)^{n-1-\alpha_{n}(\tau, t)}}{\Gamma\left(n-\alpha_{n}(\tau, t)\right)} x^{(n)}(t) d \tau d t \\
& =\int_{a}^{b} x^{(n)}(t) \int_{t}^{b} \frac{(\tau-t)^{n-1-\alpha_{n}(\tau, t)}}{\Gamma\left(n-\alpha_{n}(\tau, t)\right)} y(\tau) d \tau d t=\int_{a}^{b} x^{(n)}(t)_{t} I_{b}^{n-\alpha_{n}(\cdot, \cdot)} y(t) d t
\end{aligned}
$$

Using the (usual) integrating by parts formula, we get that (4.5) is equal to

$$
-\int_{a}^{b} x^{(n-1)}(t) \frac{d}{d t} t I_{b}^{n-\alpha_{n}(\cdot, \cdot)} y(t) d t+\left[x^{(n-1)}(t) t I_{b}^{n-\alpha_{n}(\cdot, \cdot)} y(t)\right]_{t=a}^{t=b} .
$$

Integrating by parts again, we obtain

$$
\begin{aligned}
\int_{a}^{b} x^{(n-2)}(t) & \frac{d^{2}}{d t^{2}}{ }_{t} I_{b}^{n-\alpha_{n}(\cdot, \cdot)} y(t) d t \\
+ & {\left[x^{(n-1)}(t){ }_{t} I_{b}^{n-\alpha_{n}(\cdot, \cdot)} y(t)-x^{(n-2)}(t) \frac{d}{d t} t_{b}^{n-\alpha_{n}(\cdot, \cdot)} y(t)\right]_{t=a}^{t=b} . }
\end{aligned}
$$

If we repeat this process $n-2$ times more, we get

$$
\begin{array}{rl}
\int_{a}^{b} & x(t)(-1)^{n} \frac{d^{n}}{d t^{n}} I_{b}^{n-\alpha_{n}(\cdot, \cdot)} y(t) d t \\
& +\left[\sum_{k=0}^{n-1}(-1)^{k} x^{(n-1-k)}(t) \frac{d^{k}}{d t^{k}} I_{b}^{n-\alpha_{n}(\cdot, \cdot)} y(t)\right]_{t=a}^{t=b} \\
= & \int_{a}^{b} x(t)_{t} D_{b}^{\alpha_{n}(\cdot, \cdot)} y(t) d t+\left[\sum_{k=0}^{n-1}(-1)^{k} x^{(n-1-k)}(t) \frac{d^{k}}{d t^{k}} I_{b}^{n-\alpha_{n}(\cdot, \cdot)} y(t)\right]_{t=a}^{t=b} .
\end{array}
$$

The second relation of the theorem for the right Caputo fractional derivative of order $\alpha_{n}(\cdot, \cdot)$, follows directly from the first by Caputo-Torres duality (Caputo and Torres, 2015).

Remark 43 If we consider in Theorem 42 the particular case when $n=1$, then the fractional integration by parts formulas take the well-known forms presented in Theorem 13. 
Remark 44 If $x$ is such that $x^{(i)}(a)=x^{(i)}(b)=0, i=0, \ldots, n-1$, then the higher-order formulas of fractional integration by parts given by Theorem 42 can be rewritten as

$$
\int_{a}^{b} y(t){ }_{a}^{C} D_{t}^{\alpha_{n}(\cdot, \cdot)} x(t) d t=\int_{a}^{b} x(t){ }_{t} D_{b}^{\alpha_{n}(\cdot, \cdot)} y(t) d t
$$

and

$$
\int_{a}^{b} y(t)_{t}^{C} D_{b}^{\alpha_{n}(\cdot, \cdot)} x(t) d t=\int_{a}^{b} x(t){ }_{a} D_{t}^{\alpha_{n}(\cdot, \cdot)} y(t) d t
$$

\subsection{Fundamental variational problem}

This section is dedicated to establish necessary optimality conditions for variational problems with a Lagrangian depending on a combined Caputo derivative of variable fractional order. The problem is then stated in Section 4.2.1. consisting of the variational functional

$$
\mathcal{J}(x, T)=\int_{a}^{T} L\left(t, x(t),{ }^{C} D_{\gamma}^{\alpha(\cdot, \cdot), \beta(\cdot, \cdot)} x(t)\right) d t+\phi(T, x(T)),
$$

where ${ }^{C} D_{\gamma}^{\alpha(\cdot, \cdot), \beta(\cdot, \cdot)} x(t)$ stands for the combined Caputo fractional derivative of variable fractional order (Definition 33), subject to the boundary condition $x(a)=x_{a}$.

In this problem, we do not only assume that $x(T)$ is free, but the endpoint $T$ is also variable. Therefore, we are interested in finding an optimal curve $x(\cdot)$ and also the endpoint of the variational integral, denoted in the sequel by $T$.

We begin by proving in Section 4.2.1 necessary optimality conditions that every extremizer $(x, T)$ must satisfy. The main results of this section provide necessary optimality conditions of Euler-Lagrange type, described by fractional differential equations of variable-order, and different transversality optimality conditions (Theorems 45] and 46). Some particular cases of interest are considered in Section 4.2.2 We end with two illustrative examples (Section 4.2.3).

\subsubsection{Necessary optimality conditions}

Let $\alpha, \beta:[a, b]^{2} \rightarrow(0,1)$ be two functions. Let $D$ denote the set

$$
D=\left\{(x, t) \in C^{1}([a, b]) \times[a, b]:{ }^{C} D_{\gamma}^{\alpha(\cdot, \cdot), \beta(\cdot, \cdot)} x \in C([a, b])\right\},
$$

endowed with the norm $\|(\cdot, \cdot)\|$ defined on the linear space $C^{1}([a, b]) \times \mathbb{R}$ by

$$
\|(x, t)\|:=\max _{a \leq t \leq b}|x(t)|+\max _{a \leq t \leq b}\left|{ }^{C} D_{\gamma}^{\alpha(\cdot, \cdot), \beta(\cdot, \cdot)} x(t)\right|+|t| .
$$


Definition 39 We say that $\left(x^{\star}, T^{\star}\right) \in D$ is a local minimizer to the functional $\mathcal{J}: D \rightarrow \mathbb{R}$ if there exists some $\epsilon>0$ such that

$$
\forall(x, T) \in D: \quad\left\|\left(x^{\star}, T^{\star}\right)-(x, T)\right\|<\epsilon \Rightarrow J\left(x^{\star}, T^{\star}\right) \leq J(x, T) .
$$

Along the work, we denote by $\partial_{i} z, i \in\{1,2,3\}$, the partial derivative of a function $z: \mathbb{R}^{3} \rightarrow \mathbb{R}$ with respect to its $i$ th argument, and by $L$ a differentiable Lagrangian $L:[a, b] \times \mathbb{R}^{2} \rightarrow \mathbb{R}$.

Consider the following problem of the calculus of variations:

Problem 1 Find the local minimizers of the functional $\mathcal{J}: D \rightarrow \mathbb{R}$, with

$$
\mathcal{J}(x, T)=\int_{a}^{T} L\left(t, x(t),{ }^{C} D_{\gamma}^{\alpha(\cdot, \cdot), \beta(\cdot, \cdot)} x(t)\right) d t+\phi(T, x(T)),
$$

over all $(x, T) \in D$ satisfying the boundary condition $x(a)=x_{a}$, for a fixed $x_{a} \in \mathbb{R}$. The terminal time $T$ and terminal state $x(T)$ are free.

The terminal cost function $\phi:[a, b] \times \mathbb{R} \rightarrow \mathbb{R}$ is at least of class $C^{1}$.

For simplicity of notation, we introduce the operator $[\cdot]_{\gamma}^{\alpha, \beta}$ defined by

$$
[x]_{\gamma}^{\alpha, \beta}(t)=\left(t, x(t),{ }^{C} D_{\gamma}^{\alpha(\cdot, \cdot), \beta(\cdot, \cdot)} x(t)\right) .
$$

With the new notation, one can write (4.7) simply as

$$
\mathcal{J}(x, T)=\int_{a}^{T} L[x]_{\gamma}^{\alpha, \beta}(t) d t+\phi(T, x(T)) .
$$

The next theorem gives fractional necessary optimality conditions to Problem 1

Theorem 45 Suppose that $(x, T)$ is a local minimizer to the functional (4.7) on D. Then, $(x, T)$ satisfies the fractional Euler-Lagrange equations

$$
\partial_{2} L[x]_{\gamma}^{\alpha, \beta}(t)+D_{\bar{\gamma}}^{\beta(\cdot, \cdot), \alpha(\cdot, \cdot)} \partial_{3} L[x]_{\gamma}^{\alpha, \beta}(t)=0,
$$

on the interval $[a, T]$, and

$$
\gamma_{2}\left({ }_{a} D_{t}^{\beta(\cdot, \cdot)} \partial_{3} L[x]_{\gamma}^{\alpha, \beta}(t)-{ }_{T} D_{t}^{\beta(\cdot, \cdot)} \partial_{3} L[x]_{\gamma}^{\alpha, \beta}(t)\right)=0,
$$

on the interval $[T, b]$. Moreover, $(x, T)$ satisfies the transversality conditions

$$
\left\{\begin{array}{l}
L[x]_{\gamma}^{\alpha, \beta}(T)+\partial_{1} \phi(T, x(T))+\partial_{2} \phi(T, x(T)) x^{\prime}(T)=0, \\
{\left[\gamma_{1} I_{T}^{1-\alpha(\cdot, \cdot)} \partial_{3} L[x]_{\gamma}^{\alpha, \beta}(t)-\gamma_{2} I_{t}^{1-\beta(\cdot, \cdot)} \partial_{3} L[x]_{\gamma}^{\alpha, \beta}(t)\right]_{t=T}+\partial_{2} \phi(T, x(T))=0,} \\
\gamma_{2}\left[{ }_{T} I_{t}^{1-\beta(\cdot, \cdot)} \partial_{3} L[x]_{\gamma}^{\alpha, \beta}(t)-{ }_{a} I_{t}^{1-\beta(\cdot, \cdot)} \partial_{3} L[x]_{\gamma}^{\alpha, \beta}(t)\right]_{t=b}=0 .
\end{array}\right.
$$


Proof. Let $(x, T)$ be a solution to the problem and $(x+\epsilon h, T+\epsilon \Delta T)$ be an admissible variation, where $h \in C^{1}([a, b] ; \mathbb{R})$ is a perturbing curve, $\triangle T \in \mathbb{R}$ represents an arbitrarily chosen small change in $T$ and $\epsilon \in \mathbb{R}$ represents a small number. The constraint $x(a)=x_{a}$ implies that all admissible variations must fulfill the condition $h(a)=0$. Define $j(\cdot)$ on a neighborhood of zero by

$$
\begin{aligned}
j(\epsilon) & =\mathcal{J}(x+\epsilon h, T+\epsilon \triangle T) \\
& =\int_{a}^{T+\epsilon \triangle T} L[x+\epsilon h]_{\gamma}^{\alpha, \beta}(t) d t+\phi(T+\epsilon \triangle T,(x+\epsilon h)(T+\epsilon \triangle T)) .
\end{aligned}
$$

The derivative $j^{\prime}(\epsilon)$ is

$$
\begin{aligned}
& \int_{a}^{T+\epsilon \Delta T}\left(\partial_{2} L[x+\epsilon h]_{\gamma}^{\alpha, \beta}(t) h(t)+\partial_{3} L[x+\epsilon h]_{\gamma}^{\alpha, \beta}(t)^{C} D_{\gamma}^{\alpha(\cdot, \cdot), \beta(\cdot, \cdot)} h(t)\right) d t \\
& \quad+L[x+\epsilon h]_{\gamma}^{\alpha, \beta}(T+\epsilon \Delta T) \Delta T+\partial_{1} \phi(T+\epsilon \triangle T,(x+\epsilon h)(T+\epsilon \triangle T)) \Delta T \\
& \quad+\partial_{2} \phi(T+\epsilon \triangle T,(x+\epsilon h)(T+\epsilon \triangle T))[(x+\epsilon h)(T+\epsilon \triangle T)]^{\prime} .
\end{aligned}
$$

Considering the differentiability properties of $j$, a necessary condition for $(x, T)$ to be a local extremizer is given by $\left.j^{\prime}(\epsilon)\right|_{\epsilon=0}=0$, that is,

$$
\begin{gathered}
\int_{a}^{T}\left(\partial_{2} L[x]_{\gamma}^{\alpha, \beta}(t) h(t)+\partial_{3} L[x]_{\gamma}^{\alpha, \beta}(t)^{C} D_{\gamma}^{\alpha(\cdot, \cdot), \beta(\cdot, \cdot)} h(t)\right) d t+L[x]_{\gamma}^{\alpha, \beta}(T) \Delta T \\
+\partial_{1} \phi(T, x(T)) \Delta T+\partial_{2} \phi(T, x(T))\left[h(T)+x^{\prime}(T) \Delta T\right]=0 .
\end{gathered}
$$

The second addend of the integral function (4.12),

$$
\int_{a}^{T} \partial_{3} L[x]_{\gamma}^{\alpha, \beta}(t)^{C} D_{\gamma}^{\alpha(\cdot, \cdot), \beta(\cdot, \cdot)} h(t) d t
$$

can be written, using the definition of combined Caputo fractional derivative, as

$$
\begin{aligned}
\int_{a}^{T} \partial_{3} L[x]_{\gamma}^{\alpha, \beta}(t)^{C} D_{\gamma}^{\alpha(\cdot, \cdot), \beta(\cdot, \cdot)} h(t) d t \\
=\int_{a}^{T} \partial_{3} L[x]_{\gamma}^{\alpha, \beta}(t)\left[\gamma_{1}{ }_{a}^{C} D_{t}^{\alpha(\cdot, \cdot)} h(t)+\gamma_{2}{ }_{t}^{C} D_{b}^{\beta(\cdot, \cdot)} h(t)\right] d t \\
=\gamma_{1} \int_{a}^{T} \partial_{3} L[x]_{\gamma}^{\alpha, \beta}(t)_{a}^{C} D_{t}^{\alpha(\cdot, \cdot)} h(t) d t \\
\quad+\gamma_{2}\left[\int_{a}^{b} \partial_{3} L[x]_{\gamma}^{\alpha, \beta}(t)_{t}^{C} D_{b}^{\beta(\cdot, \cdot)} h(t) d t-\int_{T}^{b} \partial_{3} L[x]_{\gamma}^{\alpha, \beta}(t)_{t}^{C} D_{b}^{\beta(\cdot, \cdot)} h(t) d t\right] .
\end{aligned}
$$

Integrating by parts (see Theorem 13), and since $h(a)=0$, the term (4.13) can be written as 


$$
\begin{gathered}
\gamma_{1}\left[\int_{a}^{T} h(t)_{t} D_{T}^{\alpha(\cdot, \cdot)} \partial_{3} L[x]_{\gamma}^{\alpha, \beta}(t) d t+\left[h(t)_{t} I_{T}^{1-\alpha(\cdot, \cdot)} \partial_{3} L[x]_{\gamma}^{\alpha, \beta}(t)\right]_{t=T}\right] \\
+\gamma_{2}\left[\int_{a}^{b} h(t)_{a} D_{t}^{\beta(\cdot, \cdot)} \partial_{3} L[x]_{\gamma}^{\alpha, \beta}(t) d t-\left[h(t)_{a} I_{t}^{1-\beta(\cdot, \cdot)} \partial_{3} L[x]_{\gamma}^{\alpha, \beta}(t)\right]_{t=b}\right. \\
-\left(\int_{T}^{b} h(t)_{T} D_{t}^{\beta(\cdot, \cdot)} \partial_{3} L[x]_{\gamma}^{\alpha, \beta}(t) d t-\left[h(t)_{T} I_{t}^{1-\beta(\cdot, \cdot)} \partial_{3} L[x]_{\gamma}^{\alpha, \beta}(t)\right]_{t=b}\right. \\
\left.\left.+\left[h(t)_{T} I_{t}^{1-\beta(\cdot, \cdot)} \partial_{3} L[x]_{\gamma}^{\alpha, \beta}(t)\right]_{t=T}\right)\right] .
\end{gathered}
$$

Unfolding these integrals, and considering the fractional operator $D_{\bar{\gamma}}^{\beta(\cdot, \cdot), \alpha(\cdot, \cdot)}$ with $\bar{\gamma}=\left(\gamma_{2}, \gamma_{1}\right)$, then (4.13) is equivalent to

$$
\begin{aligned}
\int_{a}^{T} h(t) & D_{\bar{\gamma}}^{\beta(\cdot, \cdot), \alpha(\cdot, \cdot)} \partial_{3} L[x]_{\gamma}^{\alpha, \beta}(t) d t \\
& +\int_{T}^{b} \gamma_{2} h(t)\left[{ }_{a} D_{t}^{\beta(\cdot, \cdot)} \partial_{3} L[x]_{\gamma}^{\alpha, \beta}(t)-{ }_{T} D_{t}^{\beta(\cdot, \cdot)} \partial_{3} L[x]_{\gamma}^{\alpha, \beta}(t)\right] d t \\
& +\left[h(t)\left(\gamma_{1} I_{T}^{1-\alpha(\cdot, \cdot)} \partial_{3} L[x]_{\gamma}^{\alpha, \beta}(t)-\gamma_{2} I_{t}^{1-\beta(\cdot, \cdot)} \partial_{3} L[x]_{\gamma}^{\alpha, \beta}(t)\right)\right]_{t=T} \\
& +\left[h(t) \gamma_{2}\left({ }_{T} I_{t}^{1-\beta(\cdot, \cdot)} \partial_{3} L[x]_{\gamma}^{\alpha, \beta}(t)-{ }_{a} I_{t}^{1-\beta(\cdot, \cdot)} \partial_{3} L[x]_{\gamma}^{\alpha, \beta}(t)\right)\right]_{t=b} .
\end{aligned}
$$

Substituting these relations into equation (4.12), we obtain

$$
\begin{aligned}
0= & \int_{a}^{T} h(t)\left[\partial_{2} L[x]_{\gamma}^{\alpha, \beta}(t)+D_{\bar{\gamma}}^{\beta(\cdot, \cdot), \alpha(\cdot, \cdot)} \partial_{3} L[x]_{\gamma}^{\alpha, \beta}(t)\right] d t \\
& +\int_{T}^{b} \gamma_{2} h(t)\left[{ }_{a} D_{t}^{\beta(\cdot, \cdot)} \partial_{3} L[x]_{\gamma}^{\alpha, \beta}(t)-{ }_{T} D_{t}^{\beta(\cdot, \cdot)} \partial_{3} L[x]_{\gamma}^{\alpha, \beta}(t)\right] d t \\
& +h(T)\left[\gamma_{1} I_{T}^{1-\alpha(\cdot, \cdot)} \partial_{3} L[x]_{\gamma}^{\alpha, \beta}(t)-\gamma_{2} I_{t}^{1-\beta(\cdot, \cdot)} \partial_{3} L[x]_{\gamma}^{\alpha, \beta}(t)\right. \\
& \left.\quad+\partial_{2} \phi(t, x(t))\right]_{t=T} \\
& +\Delta T\left[L[x]_{\gamma}^{\alpha, \beta}(t)+\partial_{1} \phi(t, x(t))+\partial_{2} \phi(t, x(t)) x^{\prime}(t)\right]_{t=T} \\
& +h(b)\left[\gamma_{2}\left({ }_{T} I_{t}^{1-\beta(\cdot, \cdot)} \partial_{3} L[x]_{\gamma}^{\alpha, \beta}(t)-{ }_{a} I_{t}^{1-\beta(\cdot, \cdot)} \partial_{3} L[x]_{\gamma}^{\alpha, \beta}(t)\right)\right]_{t=b} .
\end{aligned}
$$

As $h$ and $\triangle T$ are arbitrary, we can choose $\triangle T=0$ and $h(t)=0$, for all $t \in[T, b]$, but $h$ is arbitrary in $t \in[a, T)$. Then, for all $t \in[a, T]$, we obtain the first necessary condition (4.9):

$$
\partial_{2} L[x]_{\gamma}^{\alpha, \beta}(t)+D_{\bar{\gamma}}^{\beta(\cdot, \cdot), \alpha(\cdot, \cdot)} \partial_{3} L[x]_{\gamma}^{\alpha, \beta}(t)=0 .
$$

Analogously, considering $\triangle T=0, h(t)=0$, for all $t \in[a, T] \cup\{b\}$, and $h$ arbitrary on $(T, b)$, we obtain the second necessary condition (4.10): 


$$
\gamma_{2}\left({ }_{a} D_{t}^{\beta(\cdot, \cdot)} \partial_{3} L[x]_{\gamma}^{\alpha, \beta}(t)-{ }_{T} D_{t}^{\beta(\cdot, \cdot)} \partial_{3} L[x]_{\gamma}^{\alpha, \beta}(t)\right)=0 .
$$

As $(x, T)$ is a solution to the necessary conditions (4.9) and (4.10), then equation (4.14) takes the form

$$
\begin{aligned}
0= & h(T)\left[\gamma_{1} I_{T}^{1-\alpha(\cdot, \cdot)} \partial_{3} L[x]_{\gamma}^{\alpha, \beta}(t)-\gamma_{2} I_{t}^{1-\beta(\cdot, \cdot)} \partial_{3} L[x]_{\gamma}^{\alpha, \beta}(t)+\partial_{2} \phi(t, x(t))\right]_{t=T} \\
& +\Delta T\left[L[x]_{\gamma}^{\alpha, \beta}(t)+\partial_{1} \phi(t, x(t))+\partial_{2} \phi(t, x(t)) x^{\prime}(t)\right]_{t=T} \\
& +h(b)\left[\gamma_{2}\left({ }_{T} I_{t}^{1-\beta(\cdot, \cdot)} \partial_{3} L[x]_{\gamma}^{\alpha, \beta}(t)-{ }_{a} I_{t}^{1-\beta(\cdot, \cdot)} \partial_{3} L[x]_{\gamma}^{\alpha, \beta}(t)\right)\right]_{t=b} .
\end{aligned}
$$

Transversality conditions (4.11) are obtained for appropriate choices of variations.

In the next theorem, considering the same Problem 1, we rewrite the transversality conditions (4.11) in terms of the increment $\Delta T$ on time and on the consequent increment $\Delta x_{T}$ on $x$, given by

$$
\Delta x_{T}=(x+h)(T+\Delta T)-x(T) .
$$

Theorem 46 Let $(x, T)$ be a local minimizer to the functional (4.7) on $D$. Then, the fractional Euler-Lagrange equations (4.9) and (4.10) are satisfied together with the following transversality conditions:

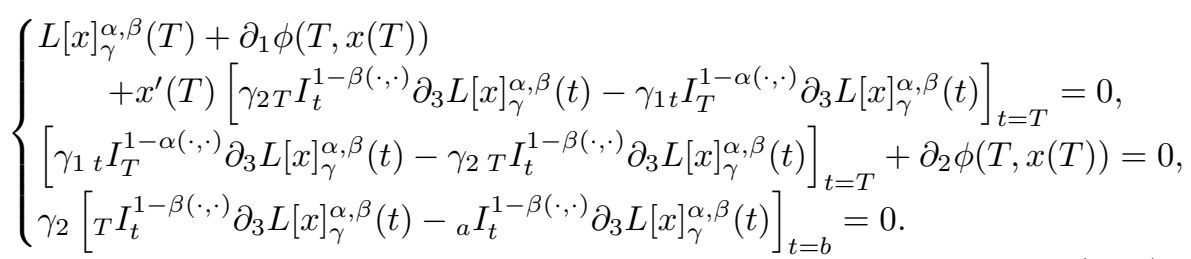

Proof. The Euler-Lagrange equations are deduced following similar arguments as the ones presented in Theorem 45. We now focus our attention on the proof of the transversality conditions. Using Taylor's expansion up to first order for a small $\Delta T$, and restricting the set of variations to those for which $h^{\prime}(T)=0$, we obtain

$$
(x+h)(T+\Delta T)=(x+h)(T)+x^{\prime}(T) \Delta T+O(\Delta T)^{2} .
$$

Rearranging the relation (4.16) allows us to express $h(T)$ in terms of $\Delta T$ and $\Delta x_{T}$ :

$$
h(T)=\Delta x_{T}-x^{\prime}(T) \Delta T+O(\Delta T)^{2} .
$$

Substitution of this expression into (4.15) gives us 


$$
\begin{aligned}
0= & \Delta x_{T}\left[\gamma_{1} I_{T}^{1-\alpha(\cdot, \cdot)} \partial_{3} L[x]_{\gamma}^{\alpha, \beta}(t)-\gamma_{2} I_{t}^{1-\beta(\cdot, \cdot)} \partial_{3} L[x]_{\gamma}^{\alpha, \beta}(t)+\partial_{2} \phi(t, x(t))\right]_{t=T} \\
+ & \Delta T\left[L[x]_{\gamma}^{\alpha, \beta}(t)+\partial_{1} \phi(t, x(t))\right. \\
& \left.\quad-x^{\prime}(t)\left(\gamma_{1} I_{T}^{1-\alpha(\cdot, \cdot)} \partial_{3} L[x]_{\gamma}^{\alpha, \beta}(t)-\gamma_{2} I_{t}^{1-\beta(\cdot, \cdot)} \partial_{3} L[x]_{\gamma}^{\alpha, \beta}(t)\right)\right]_{t=T} \\
& +h(b)\left[\gamma_{2}\left({ }_{T} I_{t}^{1-\beta(\cdot, \cdot)} \partial_{3} L[x]_{\gamma}^{\alpha, \beta}(t)-{ }_{a} I_{t}^{1-\beta(\cdot, \cdot)} \partial_{3} L[x]_{\gamma}^{\alpha, \beta}(t)\right)\right]_{t=b}+O(\Delta T)^{2} .
\end{aligned}
$$

Transversality conditions (4.17) are obtained using appropriate choices of variations.

\subsubsection{Particular cases}

Now, we specify our results to three particular cases of variable terminal points.

Vertical terminal line

This case involves a fixed upper bound $T$. Thus, $\Delta T=0$ and, consequently, the second term in (4.15) drops out. Since $\Delta x_{T}$ is arbitrary, we obtain the following transversality conditions: if $T<b$, then

$$
\left\{\begin{array}{l}
{\left[\gamma_{1} I_{T}^{1-\alpha(\cdot, \cdot)} \partial_{3} L[x]_{\gamma}^{\alpha, \beta}(t)-\gamma_{2} I_{t}^{1-\beta(\cdot, \cdot)} \partial_{3} L[x]_{\gamma}^{\alpha, \beta}(t)\right]_{t=T}+\partial_{2} \phi(T, x(T))=0,} \\
\gamma_{2}\left[{ }_{T} I_{t}^{1-\beta(\cdot, \cdot)} \partial_{3} L[x]_{\gamma}^{\alpha, \beta}(t)-{ }_{a} I_{t}^{1-\beta(\cdot, \cdot)} \partial_{3} L[x]_{\gamma}^{\alpha, \beta}(t)\right]_{t=b}=0 ;
\end{array}\right.
$$

if $T=b$, then $\Delta x_{T}=h(b)$ and the transversality conditions reduce to

$$
\left[\gamma_{1 t} I_{b}^{1-\alpha(\cdot, \cdot)} \partial_{3} L[x]_{\gamma}^{\alpha, \beta}(t)-\gamma_{2} I_{t}^{1-\beta(\cdot, \cdot)} \partial_{3} L[x]_{\gamma}^{\alpha, \beta}(t)\right]_{t=b}+\partial_{2} \phi(b, x(b))=0 .
$$

\section{Horizontal terminal line}

In this situation, we have $\Delta x_{T}=0$ but $\Delta T$ is arbitrary. Thus, the transversality conditions are

$$
\left\{\begin{array}{c}
L[x]_{\gamma}^{\alpha, \beta}(T)+\partial_{1} \phi(T, x(T)) \\
\quad+x^{\prime}(T)\left[\gamma_{2 T} I_{t}^{1-\beta(\cdot, \cdot)} \partial_{3} L[x]_{\gamma}^{\alpha, \beta}(t)-\gamma_{1 t} I_{T}^{1-\alpha(\cdot, \cdot)} \partial_{3} L[x]_{\gamma}^{\alpha, \beta}(t)\right]_{t=T}=0, \\
\gamma_{2}\left[{ }_{T} I_{t}^{1-\beta(\cdot, \cdot)} \partial_{3} L[x]_{\gamma}^{\alpha, \beta}(t)-{ }_{a} I_{t}^{1-\beta(\cdot, \cdot)} \partial_{3} L[x]_{\gamma}^{\alpha, \beta}(t)\right]_{t=b}=0 .
\end{array}\right.
$$

\section{Terminal curve}

Now the terminal point is described by a given curve $\psi: C^{1}([a, b]) \rightarrow \mathbb{R}$, in the sense that $x(T)=\psi(T)$. From Taylor's formula, for a small arbitrary $\Delta T$, one has

$$
\Delta x(T)=\psi^{\prime}(T) \Delta T+O(\Delta T)^{2} .
$$

Hence, the transversality conditions are presented in the form

$$
\left\{\begin{array}{c}
L[x]_{\gamma}^{\alpha, \beta}(T)+\partial_{1} \phi(T, x(T))+\partial_{2} \phi(T, x(T)) \psi^{\prime}(T)+\left(x^{\prime}(T)-\psi^{\prime}(T)\right) \\
\times\left[\gamma_{2} I_{t}^{1-\beta(\cdot, \cdot)} \partial_{3} L[x]_{\gamma}^{\alpha, \beta}(t)-\gamma_{1} I_{T}^{1-\alpha(\cdot, \cdot)} \partial_{3} L[x]_{\gamma}^{\alpha, \beta}(t)\right]_{t=T}=0, \\
\gamma_{2}\left[{ }_{T} I_{t}^{1-\beta(\cdot, \cdot)} \partial_{3} L[x]_{\gamma}^{\alpha, \beta}(t)-{ }_{a} I_{t}^{1-\beta(\cdot, \cdot)} \partial_{3} L[x]_{\gamma}^{\alpha, \beta}(t)\right]_{t=b}=0 .
\end{array}\right.
$$




\subsubsection{Examples}

In this section, we show two examples to illustrate the new results. Let $\alpha(t, \tau)=\alpha(t)$ and $\beta(t, \tau)=\beta(\tau)$ be two functions depending on a variable $t$ and $\tau$ only, respectively. Consider the following fractional variational problem: to minimize the functional

$$
\begin{aligned}
\mathcal{J}(x, T)=\int_{0}^{T} & {[2 \alpha(t)-1} \\
& \left.+\left({ }^{C} D_{\gamma}^{\alpha(\cdot), \beta(\cdot)} x(t)-\frac{t^{1-\alpha(t)}}{2 \Gamma(2-\alpha(t))}-\frac{(10-t)^{1-\beta(t)}}{2 \Gamma(2-\beta(t))}\right)^{2}\right] d t
\end{aligned}
$$

for $t \in[0,10]$, subject to the initial condition $x(0)=0$ and where $\gamma=$ $\left(\gamma_{1}, \gamma_{2}\right)=(1 / 2,1 / 2)$. Simple computations show that for $\bar{x}(t)=t$, with $t \in[0,10]$, we have

$$
{ }^{C} D_{\gamma}^{\alpha(\cdot), \beta(\cdot)} \bar{x}(t)=\frac{t^{1-\alpha(t)}}{2 \Gamma(2-\alpha(t))}+\frac{(10-t)^{1-\beta(t)}}{2 \Gamma(2-\beta(t))} .
$$

For $\bar{x}(t)=t$ the functional reduces to

$$
\mathcal{J}(\bar{x}, T)=\int_{0}^{T}(2 \alpha(t)-1) d t
$$

In order to determine the optimal time $T$, we have to solve the equation $2 \alpha(T)=1$. For example, let $\alpha(t)=t^{2} / 2$. In this case, since $\mathcal{J}(x, T) \geq-2 / 3$ for all pairs $(x, T)$ and $\mathcal{J}(\bar{x}, 1)=-2 / 3$, we conclude that the (global) minimum value of the functional is $-2 / 3$, obtained for $\bar{x}$ and $T=1$. It is obvious that the two Euler-Lagrange equations (4.9) and (4.10) are satisfied when $x=\bar{x}$, since

$$
\partial_{3} L[\bar{x}]_{\gamma}^{\alpha, \beta}(t)=0 \quad \text { for all } \quad t \in[0,10] .
$$

Using this relation, together with

$$
L[\bar{x}]_{\gamma}^{\alpha, \beta}(1)=0
$$

the transversality conditions (4.11) are also verified.

For our last example, consider the functional

$$
\begin{aligned}
& \mathcal{J}(x, T)=\int_{0}^{T} {[2 \alpha(t)-1} \\
&\left.+\left({ }^{C} D_{\gamma}^{\alpha(\cdot), \beta(\cdot)} x(t)-\frac{t^{1-\alpha(t)}}{2 \Gamma(2-\alpha(t))}-\frac{(10-t)^{1-\beta(t)}}{2 \Gamma(2-\beta(t))}\right)^{3}\right] d t,
\end{aligned}
$$

where the remaining assumptions and conditions are as in the previous example. For this case, $\bar{x}(t)=t$ and $T=1$ still satisfy the necessary optimality conditions. However, we cannot assure that $(\bar{x}, 1)$ is a local minimizer to the problem. 


\subsection{Higher-order variational problems}

In this section, we intend to generalize the results obtained in Section 4.2 by considering higher-order variational problems with a Lagrangian depending on a higher-order combined Caputo derivative of variable fractional order, defined by

$$
{ }^{C} D_{\gamma^{n}}^{\alpha_{n}(\cdot, \cdot), \beta_{n}(\cdot, \cdot)} x(t)=\gamma_{1}^{n}{ }_{a}^{C} D_{t}^{\alpha_{n}(\cdot, \cdot)} x(t)+\gamma_{2}^{n}{ }_{t}^{C} D_{b}^{\beta_{n}(\cdot, \cdot)} x(t),
$$

subject to boundary conditions at the initial time $t=a$.

In Section 4.3.1, we obtain higher-order Euler-Lagrange equations and transversality conditions for the generalized variational problem with a Lagrangian depending on a combined Caputo fractional derivative of variable fractional order (Theorems 47 and 49).

One illustrative example is discussed in Section 4.3.2

\subsubsection{Necessary optimality conditions}

Let $n \in \mathbb{N}$ and $x:[a, b] \rightarrow \mathbb{R}$ be a function of class $C^{n}$. The fractional order is a continuous function of two variables, $\alpha_{n}:[a, b]^{2} \rightarrow(n-1, n)$.

Let $D$ denote the linear subspace of $C^{n}([a, b]) \times[a, b]$ such that the fractional derivative of $x,{ }^{C} D_{\gamma^{i}}^{\alpha_{i}(\cdot, \cdot), \beta_{i}(\cdot, \cdot)} x(t)$, exists and is continuous on the interval $[a, b]$ for all $i \in\{1, \ldots, n\}$. We endow $D$ with the norm

$$
\|(x, t)\|=\max _{a \leq t \leq b}|x(t)|+\max _{a \leq t \leq b} \sum_{i=1}^{n}\left|{ }^{C} D_{\gamma^{i}}^{\alpha_{i}(\cdot, \cdot), \beta_{i}(\cdot, \cdot)} x(t)\right|+|t| .
$$

Consider the following higher-order problem of the calculus of variations:

Problem 2 Minimize functional $\mathcal{J}: D \rightarrow \mathbb{R}$, where

$$
\begin{array}{r}
\mathcal{J}(x, T)=\int_{a}^{T} L\left(t, x(t),{ }^{C} D_{\gamma^{1}}^{\alpha_{1}(\cdot, \cdot), \beta_{1}(\cdot, \cdot)} x(t), \ldots,{ }^{C} D_{\gamma^{n}}^{\alpha_{n}(\cdot, \cdot), \beta_{n}(\cdot, \cdot)} x(t)\right) d t \\
+\phi(T, x(T)),
\end{array}
$$

over all $(x, T) \in D$ subject to boundary conditions

$$
x(a)=x_{a}, \quad x^{(i)}(a)=x_{a}^{i}, \quad \forall i \in\{1, \ldots, n-1\},
$$

for fixed $x_{a}, x_{a}^{1}, \ldots, x_{a}^{n-1} \in \mathbb{R}$. Here the terminal time $T$ and terminal state $x(T)$ are both free. For all $i \in\{1, \ldots, n\}, \alpha_{i}, \beta_{i}\left([a, b]^{2}\right) \subseteq(i-1, i)$ and $\gamma^{i}=$ $\left(\gamma_{1}^{i}, \gamma_{2}^{i}\right)$ is a vector. The terminal cost function $\phi:[a, b] \times \mathbb{R} \rightarrow \mathbb{R}$ is at least of class $C^{1}$. 
For simplicity of notation, we introduce the operator $[\cdot]_{\gamma}^{\alpha, \beta}$ defined by

$$
[x]_{\gamma}^{\alpha, \beta}(t)=\left(t, x(t),{ }^{C} D_{\gamma^{1}}^{\alpha_{1}(\cdot, \cdot), \beta_{1}(\cdot, \cdot)} x(t), \ldots,{ }^{C} D_{\gamma^{n}}^{\alpha_{n}(\cdot, \cdot), \beta_{n}(\cdot, \cdot)} x(t)\right) .
$$

We assume that the Lagrangian $L:[a, b] \times \mathbb{R}^{n+1} \rightarrow \mathbb{R}$ is a function of class $C^{1}$. Along the work, we denote by $\partial_{i} L, i \in\{1, \ldots, n+2\}$, the partial derivative of the Lagrangian $L$ with respect to its $i$ th argument.

Now, we can rewrite functional (4.18) as

$$
\mathcal{J}(x, T)=\int_{a}^{T} L[x]_{\gamma}^{\alpha, \beta}(t) d t+\phi(T, x(T)) .
$$

In the previous section, we obtained fractional necessary optimality conditions that every local minimizer of functional $\mathcal{J}$, with $n=1$, must fulfill. Here, we generalize those results to arbitrary values of $n, n \in \mathbb{N}$. Necessary optimality conditions for Problem 2 are presented next.

Theorem 47 Suppose that $(x, T)$ gives a minimum to functional (4.19) on $D$. Then, $(x, T)$ satisfies the following fractional Euler-Lagrange equations:

$$
\partial_{2} L[x]_{\gamma}^{\alpha, \beta}(t)+\sum_{i=1}^{n} D \frac{\beta_{i}(\cdot, \cdot), \alpha_{i}(\cdot, \cdot)}{\gamma^{i}} \partial_{i+2} L[x]_{\gamma}^{\alpha, \beta}(t)=0
$$

on the interval $[a, T]$, and

$$
\sum_{i=1}^{n} \gamma_{2}^{i}\left({ }_{a} D_{t}^{\beta_{i}(\cdot, \cdot)} \partial_{i+2} L[x]_{\gamma}^{\alpha, \beta}(t)-{ }_{T} D_{t}^{\beta_{i}(\cdot, \cdot)} \partial_{i+2} L[x]_{\gamma}^{\alpha, \beta}(t)\right)=0
$$

on the interval $[T, b]$. Moreover, $(x, T)$ satisfies the following transversality conditions:

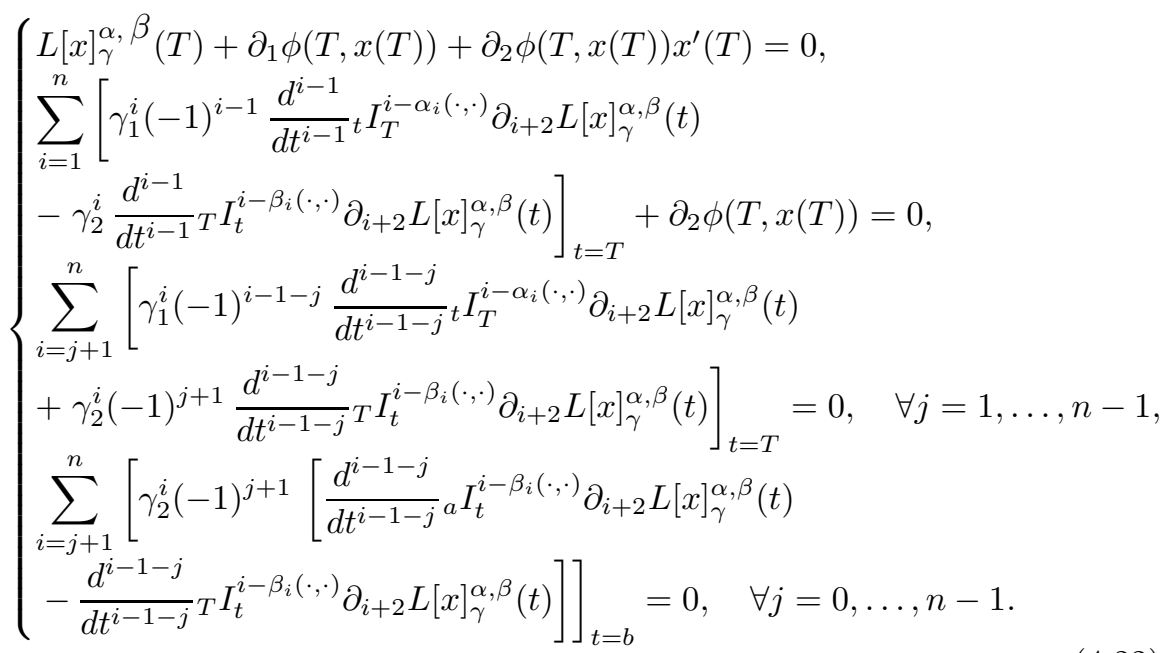


Proof. The proof is an extension of the one used in Theorem 45. Let $h \in$ $C^{n}([a, b] ; \mathbb{R})$ be a perturbing curve and $\triangle T \in \mathbb{R}$ an arbitrarily chosen small change in $T$. For a small number $\epsilon \in \mathbb{R}$, if $(x, T)$ is a solution to the problem, we consider an admissible variation of $(x, T)$ of the form $(x+\epsilon h, T+\epsilon \Delta T)$, and then, by the minimum condition, we have that

$$
\mathcal{J}(x, T) \leq \mathcal{J}(x+\epsilon h, T+\epsilon \Delta T) .
$$

The constraints $x^{(i)}(a)=x_{a}^{(i)}$ imply that all admissible variations must fulfill the conditions $h^{(i)}(a)=0$, for all $i=0, \ldots, n-1$. We define function $j(\cdot)$ on a neighborhood of zero by

$$
\begin{aligned}
j(\epsilon) & =\mathcal{J}(x+\epsilon h, T+\epsilon \triangle T) \\
& =\int_{a}^{T+\epsilon \triangle T} L[x+\epsilon h]_{\gamma}^{\alpha, \beta}(t) d t+\phi(T+\epsilon \triangle T,(x+\epsilon h)(T+\epsilon \triangle T)) .
\end{aligned}
$$

The derivative $j^{\prime}(\epsilon)$ is given by the expression

$$
\begin{aligned}
& \int_{a}^{T+\epsilon} \Delta T\left(\partial_{2} L[x+\epsilon h]_{\gamma}^{\alpha, \beta}(t) h(t)+\sum_{i=1}^{n} \partial_{i+2} L[x+\epsilon h]_{\gamma}^{\alpha, \beta}(t)^{C} D_{\gamma^{i}}^{\alpha_{i}(\cdot, \cdot), \beta_{i}(\cdot, \cdot)} h(t)\right) d t \\
&+L[x+\epsilon h]_{\gamma}^{\alpha, \beta}(T+\epsilon \Delta T) \Delta T+\partial_{1} \phi(T+\epsilon \triangle T,(x+\epsilon h)(T+\epsilon \triangle T)) \Delta T \\
&+\partial_{2} \phi(T+\epsilon \triangle T,(x+\epsilon h)(T+\epsilon \triangle T))(x+\epsilon h)^{\prime}(T+\epsilon \triangle T)
\end{aligned}
$$

Hence, by Fermat's theorem, a necessary condition for $(x, T)$ to be a local minimizer of $j$ is given by $j^{\prime}(0)=0$, that is,

$$
\begin{aligned}
& \int_{a}^{T}\left(\partial_{2} L[x]_{\gamma}^{\alpha, \beta}(t) h(t)+\sum_{i=1}^{n} \partial_{i+2} L[x]_{\gamma}^{\alpha, \beta}(t)^{C} D_{\gamma^{i}}^{\alpha_{i}(\cdot, \cdot), \beta_{i}(\cdot, \cdot)} h(t)\right) d t \\
+ & L[x]_{\gamma}^{\alpha, \beta}(T) \Delta T+\partial_{1} \phi(T, x(T)) \Delta T+\partial_{2} \phi(T, x(T))\left[h(t)+x^{\prime}(T) \Delta T\right]=0 .
\end{aligned}
$$

Considering the second addend of the integral function (4.23), for $i=1$, we get

$$
\begin{aligned}
& \int_{a}^{T} \partial_{3} L[x]_{\gamma}^{\alpha, \beta}(t)^{C} D_{\gamma^{1}}^{\alpha_{1}(\cdot, \cdot), \beta_{1}(\cdot, \cdot)} h(t) d t \\
& =\int_{a}^{T} \partial_{3} L[x]_{\gamma}^{\alpha, \beta}(t)\left[\gamma_{1}^{1}{ }_{a}^{C} D_{t}^{\alpha_{1}(\cdot, \cdot)} h(t)+\gamma_{2}^{1}{ }_{t}^{C} D_{b}^{\beta_{1}(\cdot, \cdot)} h(t)\right] d t \\
& =\gamma_{1}^{1} \int_{a}^{T} \partial_{3} L[x]_{\gamma}^{\alpha, \beta}(t)_{a}^{C} D_{t}^{\alpha_{1}(\cdot, \cdot)} h(t) d t \\
& \quad+\gamma_{2}^{1}\left[\int_{a}^{b} \partial_{3} L[x]_{\gamma}^{\alpha, \beta}(t)_{t}^{C} D_{b}^{\beta_{1}(\cdot, \cdot)} h(t) d t-\int_{T}^{b} \partial_{3} L[x]_{\gamma}^{\alpha, \beta}(t)_{t}^{C} D_{b}^{\beta_{1}(\cdot, \cdot)} h(t) d t\right] .
\end{aligned}
$$


Integrating by parts (see Theorem 42), and since $h(a)=0$, we obtain that

$$
\begin{aligned}
\gamma_{1}^{1} & {\left[\int_{a}^{T} h(t)_{t} D_{T}^{\alpha_{1}(\cdot, \cdot)} \partial_{3} L[x]_{\gamma}^{\alpha, \beta}(t) d t+\left[h(t)_{t} I_{T}^{1-\alpha_{1}(\cdot, \cdot)} \partial_{3} L[x]_{\gamma}^{\alpha, \beta}(t)\right]_{t=T}\right] } \\
& +\gamma_{2}^{1}\left[\int_{a}^{b} h(t)_{a} D_{t}^{\beta_{1}(\cdot, \cdot)} \partial_{3} L[x]_{\gamma}^{\alpha, \beta}(t) d t-\left[h(t)_{a} I_{t}^{1-\beta_{1}(\cdot, \cdot)} \partial_{3} L[x]_{\gamma}^{\alpha, \beta}(t)\right]_{t=b}\right. \\
& -\left(\int_{T}^{b} h(t)_{T} D_{t}^{\beta_{1}(\cdot, \cdot)} \partial_{3} L[x]_{\gamma}^{\alpha, \beta}(t) d t-\left[h(t)_{T} I_{t}^{1-\beta_{1}(\cdot, \cdot)} \partial_{3} L[x]_{\gamma}^{\alpha, \beta}(t)\right]_{t=b}\right. \\
& \left.\left.+\left[h(t)_{T} I_{t}^{1-\beta_{1}(\cdot, \cdot)} \partial_{3} L[x]_{\gamma}^{\alpha, \beta}(t)\right]_{t=T}\right)\right] .
\end{aligned}
$$

Unfolding these integrals, and considering the fractional operator $D{\frac{\beta_{1}, \alpha_{1}}{\gamma^{1}}}^{\text {with }}$ $\overline{\gamma^{1}}=\left(\gamma_{2}^{1}, \gamma_{1}^{1}\right)$, then the previous term is equal to

$$
\begin{aligned}
\int_{a}^{T} h(t) & D_{\gamma^{1}}^{\beta_{1}(\cdot, \cdot), \alpha_{1}(\cdot, \cdot)} \partial_{3} L[x]_{\gamma}^{\alpha, \beta}(t) d t \\
& +\int_{T}^{b} \gamma_{2}^{1} h(t)\left[{ }_{a} D_{t}^{\beta_{1}(\cdot, \cdot)} \partial_{3} L[x]_{\gamma}^{\alpha, \beta}(t)-{ }_{T} D_{t}^{\beta_{1}(\cdot, \cdot)} \partial_{3} L[x]_{\gamma}^{\alpha, \beta}(t)\right] d t \\
& +h(T)\left[\gamma_{1}^{1} I_{T}^{1-\alpha_{1}(\cdot, \cdot)} \partial_{3} L[x]_{\gamma}^{\alpha, \beta}(t)-\gamma_{2}^{1} I_{t}^{1-\beta_{1}(\cdot, \cdot)} \partial_{3} L[x]_{\gamma}^{\alpha, \beta}(t)\right]_{t=T} \\
& -h(b) \gamma_{2}^{1}\left[{ }_{a} I_{t}^{1-\beta_{1}(\cdot, \cdot)} \partial_{3} L[x]_{\gamma}^{\alpha, \beta}(t)-{ }_{T} I_{t}^{1-\beta_{1}(\cdot, \cdot)} \partial_{3} L[x]_{\gamma}^{\alpha, \beta}(t)\right]_{t=b} .
\end{aligned}
$$

Considering the third addend of the integral function (4.23), for $i=2$, we get

$$
\begin{aligned}
& \int_{a}^{T} \partial_{4} L[x]_{\gamma}^{\alpha, \beta}(t)^{C} D_{\gamma^{2}}^{\alpha_{2}(\cdot, \cdot), \beta_{2}(\cdot, \cdot)} h(t) d t=\gamma_{1}^{2} \int_{a}^{T} \partial_{4} L[x]_{\gamma}^{\alpha, \beta}(t)_{a}^{C} D_{t}^{\alpha_{2}(\cdot, \cdot)} h(t) d t \\
& +\gamma_{2}^{2}\left[\int_{a}^{b} \partial_{4} L[x]_{\gamma}^{\alpha, \beta}(t)_{t}^{C} D_{b}^{\beta_{2}(\cdot, \cdot)} h(t) d t-\int_{T}^{b} \partial_{4} L[x]_{\gamma}^{\alpha, \beta}(t)_{t}^{C} D_{b}^{\beta_{2}(\cdot, \cdot)} h(t) d t\right] \\
& =\gamma_{1}^{2}\left[\int_{a}^{T} h(t)_{t} D_{T}^{\alpha_{2}(\cdot, \cdot)} \partial_{4} L[x]_{\gamma}^{\alpha, \beta}(t) d t\right. \\
& \left.\quad+\left[h^{(1)}(t)_{t} I_{T}^{2-\alpha_{2}(\cdot, \cdot)} \partial_{4} L[x]_{\gamma}^{\alpha, \beta}(t)-h(t) \frac{d}{d t} t_{T}^{2-\alpha_{2}(\cdot, \cdot)} \partial_{4} L[x]_{\gamma}^{\alpha, \beta}(t)\right]_{t=T}\right] \\
& \quad+\gamma_{2}^{2}\left[\int_{a}^{b} h(t)_{a} D_{t}^{\beta_{2}(\cdot, \cdot)} \partial_{4} L[x]_{\gamma}^{\alpha, \beta}(t) d t\right. \\
& \quad+\left[h^{(1)}(t)_{a} I_{t}^{2-\beta_{2}(\cdot, \cdot)} \partial_{4} L[x]_{\gamma}^{\alpha, \beta}(t)-h(t) \frac{d}{d t}{ }^{a} I_{t}^{2-\beta_{2}(\cdot, \cdot)} \partial_{4} L[x]_{\gamma}^{\alpha, \beta}(t)\right]_{t=b}
\end{aligned}
$$




$$
\begin{aligned}
& -\int_{T}^{b} h(t)_{T} D_{t}^{\beta_{2}(\cdot, \cdot)} \partial_{4} L[x]_{\gamma}^{\alpha, \beta}(t) d t \\
& -\left[h^{(1)}(t)_{T} I_{t}^{2-\beta_{2}(\cdot, \cdot)} \partial_{4} L[x]_{\gamma}^{\alpha, \beta}(t)-h(t) \frac{d}{d t} T^{T} I_{t}^{2-\beta_{2}(\cdot, \cdot)} \partial_{4} L[x]_{\gamma}^{\alpha, \beta}(t)\right]_{t=b} \\
& \left.+\left[h^{(1)}(t)_{T} I_{t}^{2-\beta_{2}(\cdot, \cdot)} \partial_{4} L[x]_{\gamma}^{\alpha, \beta}(t)-h(t) \frac{d}{d t} T_{t}^{2-\beta_{2}(\cdot, \cdot)} \partial_{4} L[x]_{\gamma}^{\alpha, \beta}(t)\right]_{t=T}\right] .
\end{aligned}
$$

Again, with the auxiliary operator $D{\frac{\beta_{2}, \alpha_{2}}{\gamma^{2}}}^{\text {, with }} \overline{\gamma^{2}}=\left(\gamma_{2}^{2}, \gamma_{1}^{2}\right)$, we obtain

$$
\begin{array}{rl}
\int_{a}^{T} & h(t) D_{\gamma^{2}}^{\beta_{2}(\cdot, \cdot), \alpha_{2}(\cdot, \cdot)} \partial_{4} L[x]_{\gamma}^{\alpha, \beta}(t) d t \\
& +\int_{T}^{b} \gamma_{2}^{2} h(t)\left[{ }_{a} D_{t}^{\beta_{2}(\cdot, \cdot)} \partial_{4} L[x]_{\gamma}^{\alpha, \beta}(t)-{ }_{T} D_{t}^{\beta_{2}(\cdot, \cdot)} \partial_{4} L[x]_{\gamma}^{\alpha, \beta}(t)\right] d t \\
& +\left[h^{(1)}(t)\left(\gamma_{1}^{2} I_{T}^{2-\alpha_{2}(\cdot, \cdot)} \partial_{4} L[x]_{\gamma}^{\alpha, \beta}(t)+\gamma_{2}^{2}{ }_{T} I_{t}^{2-\beta_{2}(\cdot, \cdot)} \partial_{4} L[x]_{\gamma}^{\alpha, \beta}(t)\right)\right]_{t=T} \\
& -\left[h(t)\left(\gamma_{1}^{2} \frac{d}{d t} I_{T}^{2-\alpha_{2}(\cdot, \cdot)} \partial_{4} L[x]_{\gamma}^{\alpha, \beta}(t)+\gamma_{2}^{2} \frac{d}{d t} T_{t} I_{t}^{2-\beta_{2}(\cdot, \cdot)} \partial_{4} L[x]_{\gamma}^{\alpha, \beta}(t)\right)\right]_{t=T} \\
\quad+\left[h^{(1)}(t) \gamma_{2}^{2}\left({ }_{a} I_{t}^{2-\beta_{2}(\cdot, \cdot)} \partial_{4} L[x]_{\gamma}^{\alpha, \beta}(t)-{ }_{T} I_{t}^{2-\beta_{2}(\cdot, \cdot)} \partial_{4} L[x]_{\gamma}^{\alpha, \beta}(t)\right)\right]_{t=b} \\
\quad-\left[h(t) \gamma_{2}^{2}\left(\frac{d}{d t} I_{t}^{2-\beta_{2}(\cdot, \cdot)} \partial_{4} L[x]_{\gamma}^{\alpha, \beta}(t)-\frac{d}{d t} T^{T} I_{t}^{2-\beta_{2}(\cdot, \cdot)} \partial_{4} L[x]_{\gamma}^{\alpha, \beta}(t)\right)\right]_{t=b} .
\end{array}
$$

Now, consider the general case

$$
\int_{a}^{T} \partial_{i+2} L[x]_{\gamma}^{\alpha, \beta}(t)^{C} D_{\gamma^{i}}^{\alpha_{i}(\cdot, \cdot), \beta_{i}(\cdot, \cdot)} h(t) d t
$$

$i=3, \ldots, n$. Then, we obtain

$$
\begin{array}{rl}
\gamma_{1}^{i}\left[\int_{a}^{T} h(t)_{t} D_{T}^{\alpha_{i}(\cdot, \cdot)} \partial_{i+2} L[x]_{\gamma}^{\alpha, \beta}(t) d t\right. & \\
+ & \left.\left.\left[\sum_{k=0}^{i-1}(-1)^{k} h^{(i-1-k)}(t) \frac{d^{k}}{d t^{k}} t_{T}^{i-\alpha_{i}(\cdot, \cdot)} \partial_{i+2} L[x]_{\gamma}^{\alpha, \beta}(t) t\right)\right]_{t=T}\right] \\
+\gamma_{2}^{i} & {\left[\int_{a}^{b} h(t)_{a} D_{t}^{\beta_{i}(\cdot, \cdot)} \partial_{i+2} L[x]_{\gamma}^{\alpha, \beta}(t) d t\right.} \\
+ & {\left[\sum_{k=0}^{i-1}(-1)^{i+k} h^{(i-1-k)}(t) \frac{d^{k}}{d t^{k}} I_{t}^{i-\beta_{i}(\cdot, \cdot)} \partial_{i+2} L[x]_{\gamma}^{\alpha, \beta}(t)\right]_{t=b}} \\
-\int_{T}^{b} & h(t)_{T} D_{t}^{\beta_{i}(\cdot, \cdot)} \partial_{i+2} L[x]_{\gamma}^{\alpha, \beta}(t) d t \\
& \left.-\left[\sum_{k=0}^{i-1}(-1)^{i+k} h^{(i-1-k)}(t) \frac{d^{k}}{d t^{k}} I_{t}^{i-\beta_{i}(\cdot, \cdot)} \partial_{i+2} L[x]_{\gamma}^{\alpha, \beta}(t)\right]_{t=T}^{t=b}\right] .
\end{array}
$$


Unfolding these integrals, we obtain

$$
\begin{aligned}
& \int_{a}^{T} h(t) D \frac{\beta_{i}(\cdot, \cdot), \alpha_{i}(\cdot, \cdot)}{\gamma^{i}} \partial_{i+2} L[x]_{\gamma}^{\alpha, \beta}(t) d t \\
& +\int_{T}^{b} \gamma_{2}^{i} h(t)\left[{ }_{a} D_{t}^{\beta_{i}(\cdot, \cdot)} \partial_{i+2} L[x]_{\gamma}^{\alpha, \beta}(t)-{ }_{T} D_{t}^{\beta_{i}(\cdot, \cdot)} \partial_{i+2} L[x]_{\gamma}^{\alpha, \beta}(t)\right] d t \\
& +h^{(i-1)}(T)\left[\gamma_{1}^{i}{ }_{t} I_{T}^{i-\alpha_{i}(\cdot, \cdot)} \partial_{i+2} L[x]_{\gamma}^{\alpha, \beta}(t)+\gamma_{2}^{i}(-1)^{i}{ }_{T} I_{t}^{i-\beta_{i}(\cdot, \cdot)} \partial_{i+2} L[x]_{\gamma}^{\alpha, \beta}(t)\right]_{t=T} \\
& +h^{(i-1)}(b) \gamma_{2}^{i}(-1)^{i}\left[{ }_{a} I_{t}^{i-\beta_{i}(\cdot, \cdot)} \partial_{i+2} L[x]_{\gamma}^{\alpha, \beta}(t)-{ }_{T} I_{t}^{i-\beta_{i}(\cdot, \cdot)} \partial_{i+2} L[x]_{\gamma}^{\alpha, \beta}(t)\right]_{t=b} \\
& +h^{(i-2)}(T)\left[\gamma_{1}^{i}(-1)^{1} \frac{d}{d t}{ }_{t} I_{T}^{i-\alpha_{i}(\cdot, \cdot)} \partial_{i+2} L[x]_{\gamma}^{\alpha, \beta}(t)\right. \\
& \left.+\gamma_{2}^{i}(-1)^{i+1} \frac{d}{d t} T_{t}^{i-\beta_{i}(\cdot, \cdot)} \partial_{i+2} L[x]_{\gamma}^{\alpha, \beta}(t)\right]_{t=T} \\
& +h^{(i-2)}(b) \gamma_{2}^{i}(-1)^{i+1}\left[\frac{d}{d t} a I_{t}^{i-\beta_{i}(\cdot, \cdot)} \partial_{i+2} L[x]_{\gamma}^{\alpha, \beta}(t)\right. \\
& \left.-\frac{d}{d t} T_{t}^{i-\beta_{i}(\cdot, \cdot)} \partial_{i+2} L[x]_{\gamma}^{\alpha, \beta}(t)\right]_{t=b} \\
& +\ldots+h(T)\left[\gamma_{1}^{i}(-1)^{i-1} \frac{d^{i-1}}{d t^{i-1}} t_{T}^{i-\alpha_{i}(\cdot, \cdot)} \partial_{i+2} L[x]_{\gamma}^{\alpha, \beta}(t)\right. \\
& \left.+\gamma_{2}^{i}(-1)^{2 i-1} \frac{d^{i-1}}{d t^{i-1}} T_{t}^{i-\beta_{i}(\cdot, \cdot)} \partial_{i+2} L[x]_{\gamma}^{\alpha, \beta}(t)\right]_{t=T} \\
& +h(b) \gamma_{2}^{i}(-1)^{2 i-1}\left[\frac{d^{i-1}}{d t^{i-1}} a I_{t}^{i-\beta_{i}(\cdot, \cdot)} \partial_{i+2} L[x]_{\gamma}^{\alpha, \beta}(t)\right. \\
& \left.-\frac{d^{i-1}}{d t^{i-1}}{ }_{T} I_{t}^{i-\beta_{i}(\cdot, \cdot)} \partial_{i+2} L[x]_{\gamma}^{\alpha, \beta}(t)\right]_{t=b} .
\end{aligned}
$$

Substituting all the relations into equation (4.23), we obtain that

$$
\begin{aligned}
0= & \int_{a}^{T} h(t)\left(\partial_{2} L[x]_{\gamma}^{\alpha, \beta}(t)+\sum_{i=1}^{n} D_{\gamma^{i}}^{\beta_{i}(\cdot, \cdot), \alpha_{i}(\cdot, \cdot)} \partial_{i+2} L[x]_{\gamma}^{\alpha, \beta}(t)\right) d t \\
+ & \int_{T}^{b} h(t) \sum_{i=1}^{n} \gamma_{2}^{i}\left[{ }_{a} D_{t}^{\beta_{i}(\cdot, \cdot)} \partial_{i+2} L[x]_{\gamma}^{\alpha, \beta}(t)-{ }_{T} D_{t}^{\beta_{i}(\cdot, \cdot)} \partial_{i+2} L[x]_{\gamma}^{\alpha, \beta}(t)\right] d t \\
+ & \sum_{j=0}^{n-1} h^{(j)}(T) \sum_{i=j+1}^{n}\left[\gamma_{1}^{i}(-1)^{i-1-j} \frac{d^{i-1-j}}{d t^{i-1-j}} I_{T}^{i-\alpha_{i}(\cdot, \cdot)} \partial_{i+2} L[x]_{\gamma}^{\alpha, \beta}(t)\right. \\
& \left.+\gamma_{2}^{i}(-1)^{j+1} \frac{d^{i-1-j}}{d t^{i-1-j}} I_{t}^{i-\beta_{i}(\cdot, \cdot)} \partial_{i+2} L[x]_{\gamma}^{\alpha, \beta}(t)\right]_{t=T}
\end{aligned}
$$




$$
\begin{gathered}
+\sum_{j=0}^{n-1} h^{(j)}(b) \sum_{i=j+1}^{n} \gamma_{2}^{i}(-1)^{j+1}\left[\frac{d^{i-1-j}}{d t^{i-1-j}} a I_{t}^{i-\beta_{i}(\cdot, \cdot)} \partial_{i+2} L[x]_{\gamma}^{\alpha, \beta}(t)\right. \\
\left.-\frac{d^{i-1-j}}{d t^{i-1-j}} T_{t}^{i-\beta_{i}(\cdot, \cdot)} \partial_{i+2} L[x]_{\gamma}^{\alpha, \beta}(t)\right]_{t=b}+h(T) \partial_{2} \phi(T, x(T)) \\
+\Delta T\left[L[x]_{\gamma}^{\alpha, \beta}(T)+\partial_{1} \phi(T, x(T))+\partial_{2} \phi(T, x(T)) x^{\prime}(T)\right] .
\end{gathered}
$$

We obtain the fractional Euler-Lagrange equations (4.20)-(4.21) and the transversality conditions (4.22) applying the fundamental lemma of the calculus of variations (see, e.g., (van Brunt, 2004)) for appropriate choices of variations.

Remark 48 When $n=1$, functional (4.18) takes the form

$$
\mathcal{J}(x, T)=\int_{a}^{T} L\left(t, x(t),{ }^{C} D_{\gamma}^{\alpha(\cdot, \cdot), \beta(\cdot, \cdot)} x(t)\right) d t+\phi(T, x(T)),
$$

and the fractional Euler-Lagrange equations (4.20)-(4.21) coincide with those of Theorem 45 .

Considering the increment $\Delta T$ on time $T$, and the consequent increment $\Delta x_{T}$ on $x$, given by

$$
\Delta x_{T}=(x+h)(T+\Delta T)-x(T),
$$

in the next theorem we rewrite the transversality conditions (4.22) in terms of these increments.

Theorem 49 If $(x, T)$ minimizes functional $\mathcal{J}$ defined by (4.19), then $(x, T)$ satisfies the Euler-Lagrange equations (4.20) and (4.21), and the following transversality conditions:

$$
\left\{\begin{array}{l}
\partial_{1} \phi(T, x(T))-x^{\prime}(T) \sum_{i=1}^{n}\left[\gamma_{1}^{i}(-1)^{i-1} \frac{d^{i-1}}{d t^{i-1}}{ }_{t} I_{T}^{i-\alpha_{i}(\cdot, \cdot)} \partial_{i+2} L[x]_{\gamma}^{\alpha, \beta}(t)\right. \\
\left.-\gamma_{2}^{i} \frac{d^{i-1}}{d t^{i-1}} T I_{t}^{i-\beta_{i}(\cdot, \cdot)} \partial_{i+2} L[x]_{\gamma}^{\alpha, \beta}(t)+L[x]_{\gamma}^{\alpha, \beta}(T)\right]_{t=T}=0, \\
\sum_{i=1}^{n}\left[\gamma_{1}^{i}(-1)^{i-1} \frac{d^{i-1}}{d t^{i-1}} I_{T}^{i-\alpha_{i}(\cdot, \cdot)} \partial_{i+2} L[x]_{\gamma}^{\alpha, \beta}(t)\right. \\
\left.-\gamma_{2}^{i} \frac{d^{i-1}}{d t^{i-1}} I_{t}^{i-\beta_{i}(\cdot, \cdot)} \partial_{i+2} L[x]_{\gamma}^{\alpha, \beta}(t)\right]_{t=T}+\partial_{2} \phi(T, x(T))=0, \\
\sum_{i=j+1}^{n}\left[\gamma_{1}^{i}(-1)^{i-1-j} \frac{d^{i-1-j}}{d t^{i-1-j}} I_{T}^{i-\alpha_{i}(\cdot, \cdot)} \partial_{i+2} L[x]_{\gamma}^{\alpha, \beta}(t)\right. \\
\left.+\gamma_{2}^{i}(-1)^{j+1} \frac{d^{i-1-j}}{d t^{i-1-j}} I_{t}^{i-\beta_{i}(\cdot, \cdot)} \partial_{i+2} L[x]_{\gamma}^{\alpha, \beta}(t)\right]_{t=T}=0, \quad \forall j=2, \ldots, n-1, \\
\sum_{i=j+1}^{n}\left[\gamma _ { 2 } ^ { i } ( - 1 ) ^ { j + 1 } \left[\frac{d^{i-1-j}}{d t^{i-1-j}} I_{t}^{i-\beta_{i}(\cdot, \cdot)} \partial_{i+2} L[x]_{\gamma}^{\alpha, \beta}(t)\right.\right. \\
\left.\left.-\frac{d^{i-1-j}}{d t^{i-1-j}} I_{t}^{i-\beta_{i}(\cdot, \cdot)} \partial_{i+2} L[x]_{\gamma}^{\alpha, \beta}(t)\right]\right]_{t=b}=0, \quad \forall j=0, \ldots, n-1 .
\end{array}\right.
$$


Proof. Using Taylor's expansion up to first order, and restricting the set of variations to those for which $h^{\prime}(T)=0$, we obtain that

$$
(x+h)(T+\Delta T)=(x+h)(T)+x^{\prime}(T) \Delta T+O(\Delta T)^{2} .
$$

According to the increment on $x$ given by (4.25), we get

$$
h(T)=\Delta x_{T}-x^{\prime}(T) \Delta T+O(\Delta T)^{2} .
$$

From substitution of this expression into (4.24), and by using appropriate choices of variations, we obtain the intended transversality conditions.

\subsubsection{Example}

We provide an illustrative example. It is covered by Theorem 47 .

Let $p_{n-1}(t)$ be a polynomial of degree $n-1$. If $\alpha, \beta:[0, b]^{2} \rightarrow(n-1, n)$ are the fractional orders, then ${ }^{C} D_{\gamma}^{\alpha(\cdot, \cdot), \beta(\cdot, \cdot)} p_{n-1}(t)=0$ since $p_{n-1}^{(n)}(t)=0$ for all $t$. Consider the functional

$$
\mathcal{J}(x, T)=\int_{0}^{T}\left[\left({ }^{C} D_{\gamma}^{\alpha(\cdot, \cdot), \beta(\cdot, \cdot)} x(t)\right)^{2}+\left(x(t)-p_{n-1}(t)\right)^{2}-t-1\right] d t+T^{2}
$$

subject to the initial constraints

$$
x(0)=p_{n-1}(0) \quad \text { and } \quad x^{(k)}(0)=p_{n-1}^{(k)}(0), k=1, \ldots, n-1 .
$$

Observe that, for all $t \in[0, b]$,

$$
\partial_{i} L\left[p_{n-1}\right]_{\gamma}^{\alpha, \beta}(t)=0, \quad i=2,3 .
$$

Thus, function $x \equiv p_{n-1}$ and the final time $T=1$ satisfy the necessary optimality conditions of Theorem 47. We also remark that, for any curve $x$, one has

$$
\mathcal{J}(x, T) \geq \int_{0}^{T}[-t-1] d t+T^{2}=\frac{T^{2}}{2}-T,
$$

which attains a minimum value $-1 / 2$ at $T=1$. Since $\mathcal{J}\left(p_{n-1}, 1\right)=-1 / 2$, we conclude that $\left(p_{n-1}, 1\right)$ is the (global) minimizer of $\mathcal{J}$.

\subsection{Variational problems with time delay}

In this section, we consider fractional variational problems with time delay. As mentioned in (Machado, 2011), "we verify that a fractional derivative requires an infinite number of samples capturing, therefore, all the signal history, contrary to what happens with integer order derivatives that are merely local operators. This fact motivates the evaluation of calculation strategies 
based on delayed signal samples". This subject has already been studied for constant fractional order (Almeida, 2016; Baleanu, Maaraba and Jarad, 2008; Jarad, Abdeljawad and Baleanu, 2010). However, for a variable fractional order, it is, to the authors' best knowledge, an open question. We also refer to the works (Daftardar-Geiii. Sukale and Bhalekar, 2015; Deng. Li and Lü, 2007; Lazarević and Spasić, 2009; Wang et al., 2015), where fractional differential equations are considered with a time delay.

In Section 4.4.1 we deduce necessary optimality conditions when the Lagrangian depends on a time delay (Theorem 50). One illustrative example is discussed in Section 4.4.2.

\subsubsection{Necessary optimality conditions}

For simplicity of presentation, we consider fractional orders $\alpha, \beta:[a, b]^{2} \rightarrow$ $(0,1)$. Using similar arguments, the problem can be easily generalized for higher-order derivatives. Let $\sigma>0$ and define the vector

$$
{ }_{\sigma}[x]_{\gamma}^{\alpha, \beta}(t)=\left(t, x(t),{ }^{C} D_{\gamma}^{\alpha(\cdot, \cdot), \beta(\cdot, \cdot)} x(t), x(t-\sigma)\right) .
$$

For the domain of the functional, we consider the set

$$
D_{\sigma}=\left\{(x, t) \in C^{1}([a-\sigma, b]) \times[a, b]:{ }^{C} D_{\gamma}^{\alpha(\cdot, \cdot), \beta(\cdot, \cdot)} x \in C([a, b])\right\} .
$$

Let $\mathcal{J}: D_{\sigma} \rightarrow \mathbb{R}$ be the functional defined by

$$
\mathcal{J}(x, T)=\int_{a}^{T} L_{\sigma}[x]_{\gamma}^{\alpha, \beta}(t)+\phi(T, x(T)),
$$

where we assume again that the Lagrangian $L$ and the payoff term $\phi$ are differentiable.

The optimization problem with time delay is the following:

Problem 3 Minimize functional (4.26) on $D_{\sigma}$ subject to the boundary condition

$$
x(t)=\varphi(t)
$$

for all $t \in[a-\sigma, a]$, where $\varphi$ is a given (fixed) function.

We now state and prove the Euler-Lagrange equations for this problem.

Theorem 50 Suppose that $(x, T)$ gives a local minimum to functional (4.26) on $D_{\sigma}$. If $\sigma \geq T-a$, then $(x, T)$ satisfies

$$
\partial_{2} L_{\sigma}[x]_{\gamma}^{\alpha, \beta}(t)+D_{\bar{\gamma}}^{\beta(\cdot, \cdot), \alpha(\cdot, \cdot)} \partial_{3} L_{\sigma}[x]_{\gamma}^{\alpha, \beta}(t)=0,
$$

for $t \in[a, T]$, and 


$$
\gamma_{2}\left({ }_{a} D_{t}^{\beta(\cdot, \cdot)} \partial_{3} L_{\sigma}[x]_{\gamma}^{\alpha, \beta}(t)-{ }_{T} D_{t}^{\beta(\cdot, \cdot)} \partial_{3} L_{\sigma}[x]_{\gamma}^{\alpha, \beta}(t)\right)=0,
$$

for $t \in[T, b]$. Moreover, $(x, T)$ satisfies

$$
\left\{\begin{array}{c}
L_{\sigma}[x]_{\gamma}^{\alpha, \beta}(T)+\partial_{1} \phi(T, x(T))+\partial_{2} \phi(T, x(T)) x^{\prime}(T)=0 \\
{\left[\gamma_{1} I_{T}^{1-\alpha(\cdot, \cdot)} \partial_{3} L_{\sigma}[x]_{\gamma}^{\alpha, \beta}(t)-\gamma_{2} I_{t}^{1-\beta(\cdot, \cdot)} \partial_{3} L_{\sigma}[x]_{\gamma}^{\alpha, \beta}(t)\right]_{t=T}} \\
\quad+\partial_{2} \phi(T, x(T))=0 \\
\gamma_{2}\left[{ }_{T} I_{t}^{1-\beta(\cdot, \cdot)} \partial_{3} L_{\sigma}[x]_{\gamma}^{\alpha, \beta}(t)-{ }_{a} I_{t}^{1-\beta(\cdot, \cdot)} \partial_{3} L_{\sigma}[x]_{\gamma}^{\alpha, \beta}(t)\right]_{t=b}=0 .
\end{array}\right.
$$

If $\sigma<T-a$, then Eq. (4.27) is replaced by the two following ones:

$$
\partial_{2} L_{\sigma}[x]_{\gamma}^{\alpha, \beta}(t)+D_{\bar{\gamma}}^{\beta(\cdot, \cdot), \alpha(\cdot, \cdot)} \partial_{3} L_{\sigma}[x]_{\gamma}^{\alpha, \beta}(t)+\partial_{4} L_{\sigma}[x]_{\gamma}^{\alpha, \beta}(t+\sigma)=0,
$$

for $t \in[a, T-\sigma]$, and

$$
\partial_{2} L_{\sigma}[x]_{\gamma}^{\alpha, \beta}(t)+D_{\bar{\gamma}}^{\beta(\cdot, \cdot), \alpha(\cdot, \cdot)} \partial_{3} L_{\sigma}[x]_{\gamma}^{\alpha, \beta}(t)=0,
$$

for $t \in[T-\sigma, T]$.

Proof. Consider variations of the solution $(x+\epsilon h, T+\epsilon \Delta T)$, where $h \in$ $C^{1}([a-\sigma, b] ; \mathbb{R})$ is such that $h(t)=0$ for all $t \in[a-\sigma, a]$, and $\epsilon, \triangle T$ are two reals. If we define $j(\epsilon)=\mathcal{J}(x+\epsilon h, T+\epsilon \Delta T)$, then $j^{\prime}(0)=0$, that is,

$$
\begin{gathered}
\int_{a}^{T}\left(\partial_{2} L_{\sigma}[x]_{\gamma}^{\alpha, \beta}(t) h(t)+\partial_{3} L_{\sigma}[x]_{\gamma}^{\alpha, \beta}(t)^{C} D_{\gamma}^{\alpha(\cdot, \cdot), \beta(\cdot, \cdot)} h(t)\right. \\
\left.+\partial_{4} L_{\sigma}[x]_{\gamma}^{\alpha, \beta}(t) h(t-\sigma)\right) d t+L_{\sigma}[x]_{\gamma}^{\alpha, \beta}(T) \Delta T \\
+\partial_{1} \phi(T, x(T)) \Delta T+\partial_{2} \phi(T, x(T))\left[h(T)+x^{\prime}(T) \Delta T\right]=0
\end{gathered}
$$

First, suppose that $\sigma \geq T-a$. In this case, since

$$
\int_{a}^{T} \partial_{4} L_{\sigma}[x]_{\gamma}^{\alpha, \beta}(t) h(t-\sigma) d t=\int_{a-\sigma}^{T-\sigma} \partial_{4} L_{\sigma}[x]_{\gamma}^{\alpha, \beta}(t+\sigma) h(t) d t
$$

and $h \equiv 0$ on $[a-\sigma, a]$, this term vanishes in (4.32) and we obtain Eq. (4.12). The rest of the proof is similar to the one presented in Section 4.2, Theorem 45. and we obtain (4.27)-(4.29). Suppose now that $\sigma<T-a$. In this case, we have that

$$
\begin{aligned}
\int_{a}^{T} \partial_{4} L_{\sigma}[x]_{\gamma}^{\alpha, \beta}(t) h(t-\sigma) d t & =\int_{a-\sigma}^{T-\sigma} \partial_{4} L_{\sigma}[x]_{\gamma}^{\alpha, \beta}(t+\sigma) h(t) d t \\
& =\int_{a}^{T-\sigma} \partial_{4} L_{\sigma}[x]_{\gamma}^{\alpha, \beta}(t+\sigma) h(t) d t
\end{aligned}
$$

Next, we evaluate the integral 


$$
\begin{aligned}
& \int_{a}^{T} \partial_{3} L_{\sigma}[x]_{\gamma}^{\alpha, \beta}(t)^{C} D_{\gamma}^{\alpha(\cdot, \cdot), \beta(\cdot, \cdot)} h(t) d t \\
&=\int_{a}^{T-\sigma} \partial_{3} L_{\sigma}[x]_{\gamma}^{\alpha, \beta}(t)^{C} D_{\gamma}^{\alpha(\cdot, \cdot), \beta(\cdot, \cdot)} h(t) d t \\
& \quad+\int_{T-\sigma}^{T} \partial_{3} L_{\sigma}[x]_{\gamma}^{\alpha, \beta}(t)^{C} D_{\gamma}^{\alpha(\cdot, \cdot), \beta(\cdot, \cdot)} h(t) d t .
\end{aligned}
$$

For the first integral, integrating by parts, we have

$$
\begin{aligned}
& \int_{a}^{T-\sigma} \partial_{3} L_{\sigma}[x]_{\gamma}^{\alpha, \beta}(t)^{C} D_{\gamma}^{\alpha(\cdot, \cdot), \beta(\cdot, \cdot)} h(t) d t=\gamma_{1} \int_{a}^{T-\sigma} \partial_{3} L_{\sigma}[x]_{\gamma}^{\alpha, \beta}(t)_{a}^{C} D_{t}^{\alpha(\cdot, \cdot)} h(t) d t \\
& +\gamma_{2}\left[\int_{a}^{b} \partial_{3} L_{\sigma}[x]_{\gamma}^{\alpha, \beta}(t)_{t}^{C} D_{b}^{\beta(\cdot, \cdot)} h(t) d t-\int_{T-\sigma}^{b} \partial_{3} L_{\sigma}[x]_{\gamma}^{\alpha, \beta}(t)_{t}^{C} D_{b}^{\beta(\cdot, \cdot)} h(t) d t\right] \\
& =\int_{a}^{T-\sigma} h(t)\left[\gamma_{1 t} D_{T-\sigma}^{\alpha(\cdot, \cdot)} \partial_{3} L_{\sigma}[x]_{\gamma}^{\alpha, \beta}(t)+\gamma_{2 a} D_{t}^{\beta(\cdot, \cdot)} \partial_{3} L_{\sigma}[x]_{\gamma}^{\alpha, \beta}(t)\right] d t \\
& +\int_{T-\sigma}^{b} \gamma_{2} h(t)\left[{ }_{a} D_{t}^{\beta(\cdot, \cdot)} \partial_{3} L_{\sigma}[x]_{\gamma}^{\alpha, \beta}(t)-{ }_{T-\sigma} D_{t}^{\beta(\cdot, \cdot)} \partial_{3} L_{\sigma}[x]_{\gamma}^{\alpha, \beta}(t)\right] d t \\
& +\left[h(t)\left[\gamma_{1 t} I_{T-\sigma}^{1-\alpha(\cdot, \cdot)} \partial_{3} L_{\sigma}[x]_{\gamma}^{\alpha, \beta}(t)-\gamma_{2 T-\sigma} I_{t}^{1-\beta(\cdot, \cdot)} \partial_{3} L_{\sigma}[x]_{\gamma}^{\alpha, \beta}(t)\right]\right]_{t=T-\sigma} \\
& +\left[\gamma_{2} h(t)\left[-{ }_{a} I_{t}^{1-\beta(\cdot, \cdot)} \partial_{3} L_{\sigma}[x]_{\gamma}^{\alpha, \beta}(t)+{ }_{T-\sigma} I_{t}^{1-\beta(\cdot, \cdot)} \partial_{3} L_{\sigma}[x]_{\gamma}^{\alpha, \beta}(t)\right]\right]_{t=b} .
\end{aligned}
$$

For the second integral, in a similar way, we deduce that

$$
\begin{aligned}
& \int_{T-\sigma}^{T} \partial_{3} L_{\sigma}[x]_{\gamma}^{\alpha, \beta}(t)^{C} D_{\gamma}^{\alpha(\cdot, \cdot), \beta(\cdot, \cdot)} h(t) d t \\
& =\gamma_{1}\left[\int_{a}^{T} \partial_{3} L_{\sigma}[x]_{\gamma}^{\alpha, \beta}(t)_{a}^{C} D_{t}^{\alpha(\cdot, \cdot)} h(t) d t-\int_{a}^{T-\sigma} \partial_{3} L_{\sigma}[x]_{\gamma}^{\alpha, \beta}(t)_{a}^{C} D_{t}^{\alpha(\cdot, \cdot)} h(t) d t\right] \\
& +\gamma_{2}\left[\int_{T-\sigma}^{b} \partial_{3} L_{\sigma}[x]_{\gamma}^{\alpha, \beta}(t)_{t}^{C} D_{b}^{\beta(\cdot, \cdot)} h(t) d t-\int_{T}^{b} \partial_{3} L_{\sigma}[x]_{\gamma}^{\alpha, \beta}(t)_{t}^{C} D_{b}^{\beta(\cdot, \cdot)} h(t) d t\right] \\
& =\int_{a}^{T-\sigma} \gamma_{1} h(t)\left[{ }_{t} D_{T}^{\alpha(\cdot, \cdot)} \partial_{3} L_{\sigma}[x]_{\gamma}^{\alpha, \beta}(t)-{ }_{t} D_{T-\sigma}^{\alpha(\cdot, \cdot)} \partial_{3} L_{\sigma}[x]_{\gamma}^{\alpha, \beta}(t)\right] d t \\
& +\int_{T-\sigma}^{T} h(t)\left[\gamma_{1 t} D_{T}^{\alpha(\cdot, \cdot)} \partial_{3} L_{\sigma}[x]_{\gamma}^{\alpha, \beta}(t)+\gamma_{2 T-\sigma} D_{t}^{\beta(\cdot, \cdot)} \partial_{3} L_{\sigma}[x]_{\gamma}^{\alpha, \beta}(t)\right] d t \\
& +\int_{T}^{b} \gamma_{2} h(t)\left[{ }_{T-\sigma} D_{t}^{\beta(\cdot, \cdot)} \partial_{3} L_{\sigma}[x]_{\gamma}^{\alpha, \beta}(t)-{ }_{T} D_{t}^{\beta(\cdot, \cdot)} \partial_{3} L_{\sigma}[x]_{\gamma}^{\alpha, \beta}(t)\right] d t \\
& +\left[h(t)\left[-\gamma_{1 t} I_{T-\sigma}^{1-\alpha(\cdot, \cdot)} \partial_{3} L_{\sigma}[x]_{\gamma}^{\alpha, \beta}(t)+\gamma_{2 T-\sigma} I_{t}^{1-\beta(\cdot, \cdot)} \partial_{3} L_{\sigma}[x]_{\gamma}^{\alpha, \beta}(t)\right]\right]_{t=T-\sigma} \\
& +\left[h(t)\left[\gamma_{1 t} I_{T}^{1-\alpha(\cdot, \cdot)} \partial_{3} L_{\sigma}[x]_{\gamma}^{\alpha, \beta}(t)-\gamma_{2 T} I_{t}^{1-\beta(\cdot, \cdot)} \partial_{3} L_{\sigma}[x]_{\gamma}^{\alpha, \beta}(t)\right]\right]_{t=T} \\
& +\left[\gamma_{2} h(t)\left[-T-\sigma I_{t}^{1-\beta(\cdot, \cdot)} \partial_{3} L_{\sigma}[x]_{\gamma}^{\alpha, \beta}(t)+{ }_{T} I_{t}^{1-\beta(\cdot, \cdot)} \partial_{3} L_{\sigma}[x]_{\gamma}^{\alpha, \beta}(t)\right]\right]_{t=b} .
\end{aligned}
$$


Replacing the above equalities into (4.32), we prove that

$$
\begin{aligned}
0 & =\int_{a}^{T-\sigma} h(t)\left[\partial_{2} L_{\sigma}[x]_{\gamma}^{\alpha, \beta}(t)+D_{\bar{\gamma}}^{\beta(\cdot, \cdot), \alpha(\cdot, \cdot)} \partial_{3} L_{\sigma}[x]_{\gamma}^{\alpha, \beta}(t)+\partial_{4} L_{\sigma}[x]_{\gamma}^{\alpha, \beta}(t+\sigma)\right] d t \\
& +\int_{T-\sigma}^{T} h(t)\left[\partial_{2} L_{\sigma}[x]_{\gamma}^{\alpha, \beta}(t)+D_{\bar{\gamma}}^{\beta(\cdot, \cdot), \alpha(\cdot, \cdot)} \partial_{3} L_{\sigma}[x]_{\gamma}^{\alpha, \beta}(t)\right] d t \\
& +\int_{T}^{b} \gamma_{2} h(t)\left[{ }_{a} D_{t}^{\beta(\cdot, \cdot)} \partial_{3} L_{\sigma}[x]_{\gamma}^{\alpha, \beta}(t)-{ }_{T} D_{t}^{\beta(\cdot, \cdot)} \partial_{3} L_{\sigma}[x]_{\gamma}^{\alpha, \beta}(t)\right] d t \\
& +h(T)\left[\gamma_{1} I_{T}^{1-\alpha(\cdot, \cdot)} \partial_{3} L_{\sigma}[x]_{\gamma}^{\alpha, \beta}(t)-\gamma_{2} I_{t}^{1-\beta(\cdot, \cdot)} \partial_{3} L_{\sigma}[x]_{\gamma}^{\alpha, \beta}(t)+\partial_{2} \phi(t, x(t))\right]_{t=T} \\
& +\Delta T\left[L_{\sigma}[x]_{\gamma}^{\alpha, \beta}(t)+\partial_{1} \phi(t, x(t))+\partial_{2} \phi(t, x(t)) x^{\prime}(t)\right]_{t=T} \\
& +h(b)\left[\gamma_{2}\left({ }_{T} I_{t}^{1-\beta(\cdot, \cdot)} \partial_{3} L_{\sigma}[x]_{\gamma}^{\alpha, \beta}(t)-{ }_{a} I_{t}^{1-\beta(\cdot, \cdot)} \partial_{3} L_{\sigma}[x]_{\gamma}^{\alpha, \beta}(t)\right)\right]_{t=b}
\end{aligned}
$$

By the arbitrariness of $h$ in $[a, b]$ and of $\triangle T$, we obtain Eqs. (4.28)-(4.31).

\subsubsection{Example}

Let $\alpha, \beta:[0, b]^{2} \rightarrow(0,1), \sigma=1, f$ be a function of class $C^{1}$, and $\hat{f}(t)=$ ${ }^{C} D_{\gamma}^{\alpha(\cdot, \cdot), \beta(\cdot, \cdot)} f(t)$. Consider the following problem of the calculus of variations:

$$
\begin{aligned}
\mathcal{J}(x, T)=\int_{0}^{T}\left[\left({ }^{C} D_{\gamma}^{\alpha(\cdot, \cdot), \beta(\cdot, \cdot)} x(t)-\hat{f}(t)\right)^{2}+(x(t)-f(t))^{2}\right. & \\
& \left.+(x(t-1)-f(t-1))^{2}-t-2\right] d t+T^{2} \rightarrow \min
\end{aligned}
$$

subject to the condition $x(t)=f(t)$ for all $t \in[-1,0]$.

In this case, we can easily verify that $(x, T)=(f, 2)$ satisfies all the conditions in Theorem 50 and that it is actually the (global) minimizer of the problem.

\subsection{Isoperimetric problems}

Isoperimetric problems are optimization problems, that consist in minimizing or maximizing a cost functional subject to an integral constraint. From the variational problem with dependence on a combined Caputo derivative of variable fractional order (see Definition 33) discussed in Section 4.2. here we study two variational problems subject to an additional integral constraint. In each of the problems, the terminal point in the cost integral, as well as the terminal state, are considered to be free, and we obtain corresponding natural boundary conditions.

In Sections 4.5.1 and 4.5.2 we study necessary optimality conditions in order to determine the minimizers for each of the problems. We end this section with an example (Section 4.5.3). 
For the two isoperimetric problems considered in the next sections, let $D$ be the set given by (4.6) and $[x]_{\gamma}^{\alpha, \beta}(t)$ the vector (4.8).

Picking up the problem of the fractional calculus of variations with variable-order, discussed in Section 4.2, we consider also a differentiable Lagrangian $L:[a, b] \times \mathbb{R}^{2} \rightarrow \mathbb{R}$ and the functional $\mathcal{J}: D \rightarrow \mathbb{R}$ of the form

$$
\mathcal{J}(x, T)=\int_{a}^{T} L[x]_{\gamma}^{\alpha, \beta}(t) d t+\phi(T, x(T)),
$$

where the terminal cost function $\phi:[a, b] \times \mathbb{R} \rightarrow \mathbb{R}$ is of class $C^{1}$.

In the sequel, we need the auxiliary notation of the dual fractional derivative:

$D_{\bar{\gamma}, c}^{\beta(\cdot,), \alpha(\cdot, \cdot)}=\gamma_{2}{ }_{a} D_{t}^{\beta(\cdot, \cdot)}+\gamma_{1} D_{c}^{\alpha(\cdot, \cdot)}, \quad$ where $\quad \bar{\gamma}=\left(\gamma_{2}, \gamma_{1}\right) \quad$ and $\quad c \in(a, b]$.

With the functional $\mathcal{J}$, defined by (4.33), we consider two different isoperimetrics problems.

\subsubsection{Necessary optimality conditions I}

The first fractional isoperimetric problem of the calculus of variations is Problem 4 .

Problem 4 Determine the local minimizers of $\mathcal{J}$ over all $(x, T) \in D$ satisfying a boundary condition

$$
x(a)=x_{a}
$$

for a fixed $x_{a} \in \mathbb{R}$ and an integral constraint of the form

$$
\int_{a}^{T} g[x]_{\gamma}^{\alpha, \beta}(t) d t=\psi(T)
$$

where $g: C^{1}\left([a, b] \times \mathbb{R}^{2}\right) \rightarrow \mathbb{R}$ and $\psi:[a, b] \rightarrow \mathbb{R}$ are two differentiable functions. The terminal time $T$ and terminal state $x(T)$ are free.

In this problem, the condition of the form (4.36) is called an isoperimetric constraint. The next theorem gives fractional necessary optimality conditions for Problem 4 .

Theorem 51 Suppose that $(x, T)$ gives a local minimum for functional (4.33) on D subject to the boundary condition (4.35) and the isoperimetric constraint (4.36). If $(x, T)$ does not satisfy the Euler-Lagrange equations with respect to the isoperimetric constraint, that is, if one of the two following conditions are not verified,

$$
\partial_{2} g[x]_{\gamma}^{\alpha, \beta}(t)+D_{\bar{\gamma}, T}^{\beta(\cdot, \cdot), \alpha(\cdot, \cdot)} \partial_{3} g[x]_{\gamma}^{\alpha, \beta}(t)=0, \quad t \in[a, T],
$$


or

$$
\gamma_{2}\left[{ }_{a} D_{t}^{\beta(\cdot, \cdot)} \partial_{3} g[x]_{\gamma}^{\alpha, \beta}(t)-{ }_{T} D_{t}^{\beta(\cdot, \cdot)} \partial_{3} g[x]_{\gamma}^{\alpha, \beta}(t)\right]=0, \quad t \in[T, b],
$$

then there exists a constant $\lambda$ such that, if we define the function $F:[a, b] \times$ $\mathbb{R}^{2} \rightarrow \mathbb{R}$ by $F=L-\lambda g,(x, T)$ satisfies the fractional Euler-Lagrange equations

$$
\partial_{2} F[x]_{\gamma}^{\alpha, \beta}(t)+D_{\bar{\gamma}, T}^{\beta(\cdot, \cdot), \alpha(\cdot, \cdot)} \partial_{3} F[x]_{\gamma}^{\alpha, \beta}(t)=0
$$

on the interval $[a, T]$ and

$$
\gamma_{2}\left({ }_{a} D_{t}^{\beta(\cdot, \cdot)} \partial_{3} F[x]_{\gamma}^{\alpha, \beta}(t)-{ }_{T} D_{t}^{\beta(\cdot, \cdot)} \partial_{3} F[x]_{\gamma}^{\alpha, \beta}(t)\right)=0
$$

on the interval $[T, b]$. Moreover, $(x, T)$ satisfies the transversality conditions

$$
\left\{\begin{array}{l}
F[x]_{\gamma}^{\alpha, \beta}(T)+\partial_{1} \phi(T, x(T))+\partial_{2} \phi(T, x(T)) x^{\prime}(T)+\lambda \psi^{\prime}(T)=0 \\
{\left[\gamma_{1 t} I_{T}^{1-\alpha(\cdot, \cdot)} \partial_{3} F[x]_{\gamma}^{\alpha, \beta}(t)-\gamma_{2} I_{t}^{1-\beta(\cdot, \cdot)} \partial_{3} F[x]_{\gamma}^{\alpha, \beta}(t)\right]_{t=T}+\partial_{2} \phi(T, x(T))=0} \\
\gamma_{2}\left[{ }_{T} I_{t}^{1-\beta(\cdot, \cdot)} \partial_{3} F[x]_{\gamma}^{\alpha, \beta}(t)-{ }_{a} I_{t}^{1-\beta(\cdot, \cdot)} \partial_{3} F[x]_{\gamma}^{\alpha, \beta}(t)\right]_{t=b}=0
\end{array}\right.
$$

Proof. Consider variations of the optimal solution $(x, T)$ of the type

$$
\left(x^{*}, T^{*}\right)=\left(x+\epsilon_{1} h_{1}+\epsilon_{2} h_{2}, T+\epsilon_{1} \Delta T\right),
$$

where, for each $i \in\{1,2\}, \epsilon_{i} \in \mathbb{R}$ is a small parameter, $h_{i} \in C^{1}([a, b] ; \mathbb{R})$ satisfies $h_{i}(a)=0$, and $\triangle T \in \mathbb{R}$. The additional term $\epsilon_{2} h_{2}$ must be selected so that the admissible variations $\left(x^{*}, T^{*}\right)$ satisfy the isoperimetric constraint (4.36). For a fixed choice of $h_{i}$, let

$$
i\left(\epsilon_{1}, \epsilon_{2}\right)=\int_{a}^{T+\epsilon_{1} \triangle T} g\left[x^{*}\right]_{\gamma}^{\alpha, \beta}(t) d t-\psi\left(T+\epsilon_{1} \triangle T\right) .
$$

For $\epsilon_{1}=\epsilon_{2}=0$, we obtain that

$$
i(0,0)=\int_{a}^{T} g[x]_{\gamma}^{\alpha, \beta}(t) d t-\psi(T)=\psi(T)-\psi(T)=0 .
$$

The derivative $\frac{\partial i}{\partial \epsilon_{2}}$ is given by

$$
\frac{\partial i}{\partial \epsilon_{2}}=\int_{a}^{T+\epsilon_{1} \triangle T}\left(\partial_{2} g\left[x^{*}\right]_{\gamma}^{\alpha, \beta}(t) h_{2}(t)+\partial_{3} g\left[x^{*}\right]_{\gamma}^{\alpha, \beta}(t)^{C} D_{\gamma}^{\alpha(\cdot, \cdot), \beta(\cdot, \cdot)} h_{2}(t)\right) d t
$$

For $\epsilon_{1}=\epsilon_{2}=0$ one has

$$
\left.\frac{\partial i}{\partial \epsilon_{2}}\right|_{(0,0)}=\int_{a}^{T}\left(\partial_{2} g[x]_{\gamma}^{\alpha, \beta}(t) h_{2}(t)+\partial_{3} g[x]_{\gamma}^{\alpha, \beta}(t)^{C} D_{\gamma}^{\alpha(\cdot, \cdot), \beta(\cdot, \cdot)} h_{2}(t)\right) d t .
$$


The second term in (4.43) can be written as

$$
\begin{aligned}
\int_{a}^{T} & \partial_{3} g[x]_{\gamma}^{\alpha, \beta}(t)^{C} D_{\gamma}^{\alpha(\cdot, \cdot), \beta(\cdot, \cdot)} h_{2}(t) d t \\
& =\int_{a}^{T} \partial_{3} g[x]_{\gamma}^{\alpha, \beta}(t)\left[\gamma_{1}^{C}{ }_{a}^{C} D_{t}^{\alpha(\cdot, \cdot)} h_{2}(t)+\gamma_{2}{ }_{t}^{C} D_{b}^{\beta(\cdot, \cdot)} h_{2}(t)\right] d t \\
& =\gamma_{1} \int_{a}^{T} \partial_{3} g[x]_{\gamma}^{\alpha, \beta}(t)_{a}^{C} D_{t}^{\alpha(\cdot, \cdot)} h_{2}(t) d t \\
& +\gamma_{2}\left[\int_{a}^{b} \partial_{3} g[x]_{\gamma}^{\alpha, \beta}(t)_{t}^{C} D_{b}^{\beta(\cdot, \cdot)} h_{2}(t) d t-\int_{T}^{b} \partial_{3} g[x]_{\gamma}^{\alpha, \beta}(t)_{t}^{C} D_{b}^{\beta(\cdot, \cdot)} h_{2}(t) d t\right] .
\end{aligned}
$$

Using the fractional integrating by parts formula, (4.44) is equal to

$$
\begin{aligned}
& \int_{a}^{T} h_{2}(t)\left[\gamma_{1 t} D_{T}{ }^{\alpha(\cdot, \cdot)} \partial_{3} g[x]_{\gamma}^{\alpha, \beta}(t)+\gamma_{2 a} D_{t}{ }^{\beta(\cdot, \cdot)} \partial_{3} g[x]_{\gamma}^{\alpha, \beta}(t)\right] d t \\
& \quad+\int_{T}^{b} \gamma_{2} h_{2}(t)\left[{ }_{a} D_{t}^{\beta(\cdot, \cdot)} \partial_{3} g[x]_{\gamma}^{\alpha, \beta}(t)-{ }_{T} D_{t}{ }^{\beta(\cdot, \cdot)} \partial_{3} g[x]_{\gamma}^{\alpha, \beta}(t)\right] d t \\
& \quad+\left[h_{2}(t)\left(\gamma_{1 t} I_{T}{ }^{1-\alpha(\cdot, \cdot)} \partial_{3} g[x]_{\gamma}^{\alpha, \beta}(t)-\gamma_{2 T} I_{t}{ }^{1-\beta(\cdot, \cdot)} \partial_{3} g[x]_{\gamma}^{\alpha, \beta}(t)\right)\right]_{t=T} \\
& \quad+\left[\gamma_{2} h_{2}(t)\left({ }_{T} I_{t}{ }^{1-\beta(\cdot, \cdot)} \partial_{3} g[x]_{\gamma}^{\alpha, \beta}(t)-{ }_{a} I_{t}{ }^{1-\beta(\cdot, \cdot)} \partial_{3} g[x]_{\gamma}^{\alpha, \beta}(t)\right)\right]_{t=b} .
\end{aligned}
$$

Substituting these relations into (4.43), and considering the fractional operator $D_{\bar{\gamma}, c}^{\beta(\cdot, \cdot), \alpha(\cdot, \cdot)}$ as defined in (4.34), we obtain that

$$
\begin{aligned}
\left.\frac{\partial i}{\partial \epsilon_{2}}\right|_{(0,0)} & =\int_{a}^{T} h_{2}(t)\left[\partial_{2} g[x]_{\gamma}^{\alpha, \beta}(t)+D_{\bar{\gamma}, T}^{\beta(\cdot, \cdot), \alpha(\cdot, \cdot)} \partial_{3} g[x]_{\gamma}^{\alpha, \beta}(t)\right] d t \\
& +\int_{T}^{b} \gamma_{2} h_{2}(t)\left[{ }_{a} D_{t}^{\beta(\cdot, \cdot)} \partial_{3} g[x]_{\gamma}^{\alpha, \beta}(t)-{ }_{T} D_{t}{ }^{\beta(\cdot, \cdot)} \partial_{3} g[x]_{\gamma}^{\alpha, \beta}(t)\right] d t \\
& +\left[h_{2}(t)\left(\gamma_{1 t} I_{T}{ }^{1-\alpha(\cdot, \cdot)} \partial_{3} g[x]_{\gamma}^{\alpha, \beta}(t)-\gamma_{2} I_{t}{ }^{1-\beta(\cdot, \cdot)} \partial_{3} g[x]_{\gamma}^{\alpha, \beta}(t)\right)\right]_{t=T} \\
& +\left[\gamma_{2} h_{2}(t)\left({ }_{T} I_{t}{ }^{1-\beta(\cdot, \cdot)} \partial_{3} g[x]_{\gamma}^{\alpha, \beta}(t)-{ }_{a} I_{t}{ }^{1-\beta(\cdot, \cdot)} \partial_{3} g[x]_{\gamma}^{\alpha, \beta}(t)\right)\right]_{t=b} .
\end{aligned}
$$

Since (4.37) or (4.38) fails, there exists a function $h_{2}$ such that

$$
\left.\frac{\partial i}{\partial \epsilon_{2}}\right|_{(0,0)} \neq 0
$$


In fact, if not, from the arbitrariness of the function $h_{2}$ and the fundamental lemma of the calculus of the variations, (4.37) and (4.38) would be verified. Thus, we may apply the implicit function theorem, that ensures the existence of a function $\epsilon_{2}(\cdot)$, defined in a neighborhood of zero, such that $i\left(\epsilon_{1}, \epsilon_{2}\left(\epsilon_{1}\right)\right)=$ 0 . In conclusion, there exists a subfamily of variations of the form (4.42) that verifies the integral constraint (4.36). We now seek to prove the main result. For that purpose, consider the auxiliary function $j\left(\epsilon_{1}, \epsilon_{2}\right)=\mathcal{J}\left(x^{*}, T^{*}\right)$.

By hypothesis, function $j$ attains a local minimum at $(0,0)$ when subject to the constraint $i(\cdot, \cdot)=0$, and we proved before that $\nabla i(0,0) \neq(0,0)$. Applying the Lagrange multiplier rule, we ensure the existence of a number $\lambda$ such that

$$
\nabla(j(0,0)-\lambda i(0,0))=(0,0) .
$$

In particular,

$$
\frac{\partial(j-\lambda i)}{\partial \epsilon_{1}}(0,0)=0 \text {. }
$$

Let $F=L-\lambda g$. The relation (4.45) can be written as

$$
\begin{aligned}
0 & =\int_{a}^{T} h_{1}(t)\left[\partial_{2} F[x]_{\gamma}^{\alpha, \beta}(t)+D_{\bar{\gamma}, T}^{\beta(\cdot, \cdot), \alpha(\cdot, \cdot)} \partial_{3} F[x]_{\gamma}^{\alpha, \beta}(t)\right] d t \\
& +\int_{T}^{b} \gamma_{2} h_{1}(t)\left[{ }_{a} D_{t}^{\beta(\cdot, \cdot)} \partial_{3} F[x]_{\gamma}^{\alpha, \beta}(t)-{ }_{T} D_{t}^{\beta(\cdot, \cdot)} \partial_{3} F[x]_{\gamma}^{\alpha, \beta}(t)\right] d t \\
& +h_{1}(T)\left[\gamma_{1} I_{T}^{1-\alpha(\cdot, \cdot)} \partial_{3} F[x]_{\gamma}^{\alpha, \beta}(t)-\gamma_{2} I_{t}^{1-\beta(\cdot, \cdot)} \partial_{3} F[x]_{\gamma}^{\alpha, \beta}(t)+\partial_{2} \phi(t, x(t))\right]_{t=T} \\
& +\Delta T\left[F[x]_{\gamma}^{\alpha, \beta}(t)+\partial_{1} \phi(t, x(t))+\partial_{2} \phi(t, x(t)) x^{\prime}(t)+\lambda \psi^{\prime}(t)\right]_{t=T} \\
& +h_{1}(b) \gamma_{2}\left[{ }_{T} I_{t}^{1-\beta(\cdot, \cdot)} \partial_{3} F[x]_{\gamma}^{\alpha, \beta}(t)-{ }_{a} I_{t}^{1-\beta(\cdot, \cdot)} \partial_{3} F[x]_{\gamma}^{\alpha, \beta}(t)\right]_{t=b}
\end{aligned}
$$

As $h_{1}$ and $\triangle T$ are arbitrary, we can choose $\triangle T=0$ and $h_{1}(t)=0$ for all $t \in[T, b]$. But $h_{1}$ is arbitrary in $t \in[a, T)$. Then, we obtain the first necessary condition (4.39):

$$
\partial_{2} F[x]_{\gamma}^{\alpha, \beta}(t)+D_{\bar{\gamma}, T}^{\beta(\cdot, \cdot), \alpha(\cdot, \cdot)} \partial_{3} F[x]_{\gamma}^{\alpha, \beta}(t)=0 \quad \forall t \in[a, T] .
$$

Analogously, considering $\triangle T=0$ and $h_{1}(t)=0$ for all $t \in[a, T] \cup\{b\}$, and $h_{1}$ arbitrary on $(T, b)$, we obtain the second necessary condition (4.40):

$$
\gamma_{2}\left({ }_{a} D_{t}^{\beta(\cdot, \cdot)} \partial_{3} F[x]_{\gamma}^{\alpha, \beta}(t)-{ }_{T} D_{t}^{\beta(\cdot, \cdot)} \partial_{3} F[x]_{\gamma}^{\alpha, \beta}(t)\right)=0 \quad \forall t \in[T, b] .
$$

As $(x, T)$ is a solution to the necessary conditions (4.39) and (4.40), then equation (4.46) takes the form 


$$
\begin{aligned}
& h_{1}(T)\left[\gamma_{1} I_{T}^{1-\alpha(\cdot, \cdot)} \partial_{3} F[x]_{\gamma}^{\alpha, \beta}(t)-\gamma_{2} I_{t}^{1-\beta(\cdot, \cdot)} \partial_{3} F[x]_{\gamma}^{\alpha, \beta}(t)+\partial_{2} \phi(t, x(t))\right]_{t=T} \\
& +\Delta T\left[F[x]_{\gamma}^{\alpha, \beta}(t)+\partial_{1} \phi(t, x(t))+\partial_{2} \phi(t, x(t)) x^{\prime}(t)+\lambda \psi^{\prime}(t)\right]_{t=T} \\
& +h_{1}(b)\left[\gamma_{2}\left({ }_{T} I_{t}^{1-\beta(\cdot, \cdot)} \partial_{3} F[x]_{\gamma}^{\alpha, \beta}(t)-{ }_{a} I_{t}^{1-\beta(\cdot, \cdot)} \partial_{3} F[x]_{\gamma}^{\alpha, \beta}(t)\right)\right]_{t=b}=0 .
\end{aligned}
$$

Transversality conditions (4.41) are obtained for appropriate choices of variations.

In the next theorem, considering the same Problem 4, we rewrite the transversality conditions (4.41) in terms of the increment on time $\Delta T$ and on the increment of space $\Delta x_{T}$ given by

$$
\Delta x_{T}=\left(x+h_{1}\right)(T+\Delta T)-x(T) .
$$

Theorem 52 Let $(x, T)$ be a local minimizer to the functional (4.33) on $D$ subject to the boundary condition (4.35) and the isoperimetric constraint (4.36). Then $(x, T)$ satisfies the transversality conditions

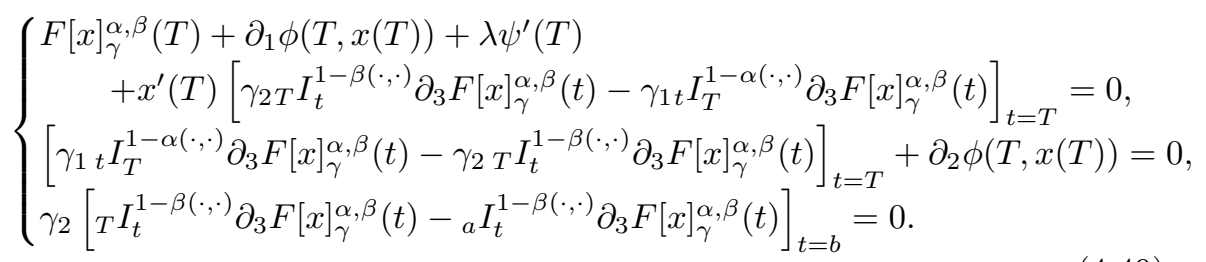

Proof. Suppose $\left(x^{*}, T^{*}\right)$ is an admissible variation of the form (4.42) with $\epsilon_{1}=1$ and $\epsilon_{2}=0$. Using Taylor's expansion up to first order for a small $\Delta T$, and restricting the set of variations to those for which $h_{1}^{\prime}(T)=0$, we obtain the increment $\Delta x_{T}$ on $x$ :

$$
\left(x+h_{1}\right)(T+\Delta T)=\left(x+h_{1}\right)(T)+x^{\prime}(T) \Delta T+O(\Delta T)^{2} .
$$

Relation (4.48) allows us to express $h_{1}(T)$ in terms of $\Delta T$ and $\Delta x_{T}$ :

$$
h_{1}(T)=\Delta x_{T}-x^{\prime}(T) \Delta T+O(\Delta T)^{2} .
$$

Substituting this expression into (4.47), and using appropriate choices of variations, we obtain the new transversality conditions (4.49).

Theorem 53 Suppose that $(x, T)$ gives a local minimum for functional (4.33) on $D$ subject to the boundary condition (4.35) and the isoperimetric constraint (4.36). Then, there exists $\left(\lambda_{0}, \lambda\right) \neq(0,0)$ such that, if we define the function $F:[a, b] \times \mathbb{R}^{2} \rightarrow \mathbb{R}$ by $F=\lambda_{0} L-\lambda g,(x, T)$ satisfies the following fractional Euler-Lagrange equations:

$$
\partial_{2} F[x]_{\gamma}^{\alpha, \beta}(t)+D_{\bar{\gamma}, T}^{\beta(\cdot, \cdot), \alpha(\cdot, \cdot)} \partial_{3} F[x]_{\gamma}^{\alpha, \beta}(t)=0
$$


on the interval $[a, T]$, and

$$
\gamma_{2}\left({ }_{a} D_{t}^{\beta(\cdot, \cdot)} \partial_{3} F[x]_{\gamma}^{\alpha, \beta}(t)-{ }_{T} D_{t}^{\beta(\cdot, \cdot)} \partial_{3} F[x]_{\gamma}^{\alpha, \beta}(t)\right)=0
$$

on the interval $[T, b]$.

Proof. If $(x, T)$ does not verifies (4.37) or (4.38), then the hypothesis of Theorem 51 is satisfied and we prove Theorem 53 considering $\lambda_{0}=1$. If $(x, T)$ verifies (4.37) and (4.38), then we prove the result by considering $\lambda=1$ and $\lambda_{0}=0$.

\subsubsection{Necessary optimality conditions II}

We now consider a new isoperimetric type problem with the isoperimetric constraint of form

$$
\int_{a}^{b} g[x]_{\gamma}^{\alpha, \beta}(t) d t=C
$$

where $C$ is a given real number.

Problem 5 Determine the local minimizers of $\mathcal{J}$ (4.33), over all $(x, T) \in D$ satisfying a boundary condition

$$
x(a)=x_{a}
$$

for a fixed $x_{a} \in \mathbb{R}$ and an integral constraint of the form (4.50).

In the follow theorem, we give fractional necessary optimality conditions for Problem 5 ,

Theorem 54 Suppose that $(x, T)$ gives a local minimum for functional (4.33) on D subject to the boundary condition (4.51) and the isoperimetric constraint (4.50). If $(x, T)$ does not satisfy the Euler-Lagrange equation with respect to the isoperimetric constraint, that is, the condition

$$
\partial_{2} g[x]_{\gamma}^{\alpha, \beta}(t)+D_{\bar{\gamma}, b}^{\beta(\cdot, \cdot), \alpha(\cdot, \cdot)} \partial_{3} g[x]_{\gamma}^{\alpha, \beta}(t)=0, \quad t \in[a, b],
$$

is not satisfied, then there exists $\lambda \neq 0$ such that, if we define the function $F:[a, b] \times \mathbb{R}^{2} \rightarrow \mathbb{R}$ by $F=L-\lambda g,(x, T)$ satisfies the fractional EulerLagrange equations

$$
\partial_{2} F[x]_{\gamma}^{\alpha, \beta}(t)+D_{\bar{\gamma}, T}^{\beta(\cdot, \cdot), \alpha(\cdot, \cdot)} \partial_{3} L[x]_{\gamma}^{\alpha, \beta}(t)-\lambda D_{\bar{\gamma}, b}^{\beta(\cdot, \cdot), \alpha(\cdot, \cdot)} \partial_{3} g[x]_{\gamma}^{\alpha, \beta}(t)=0
$$

on the interval $[a, T]$, and

$$
\begin{aligned}
\gamma_{2}\left({ }_{a} D_{t}^{\beta(\cdot, \cdot)} \partial_{3} F[x]_{\gamma}^{\alpha, \beta}(t)-{ }_{T} D_{t}^{\beta(\cdot, \cdot)} \partial_{3} L[x]_{\gamma}^{\alpha, \beta}(t)\right) \\
-\lambda\left(\partial_{2} g[x]_{\gamma}^{\alpha, \beta}(t)+\gamma_{1 t} D_{b}^{\alpha(\cdot, \cdot)} \partial_{3} g[x]_{\gamma}^{\alpha, \beta}(t)\right)=0
\end{aligned}
$$


on the interval $[T, b]$. Moreover, $(x, T)$ satisfies the transversality conditions

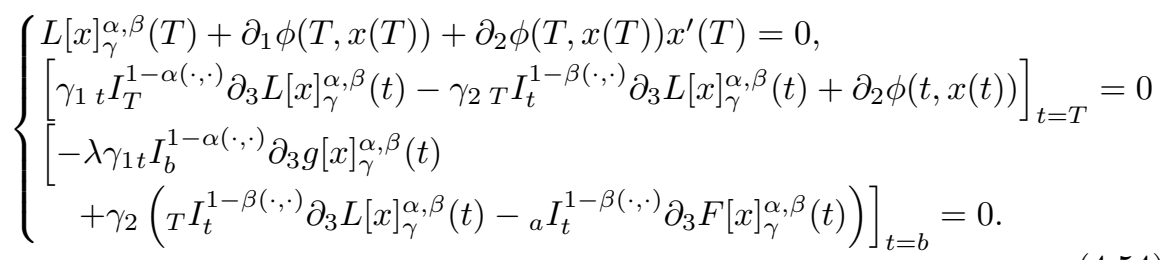

Proof. Similarly as done to prove Theorem 51, let

$$
\left(x^{*}, T^{*}\right)=\left(x+\epsilon_{1} h_{1}+\epsilon_{2} h_{2}, T+\epsilon_{1} \Delta T\right)
$$

be a variation of the solution, and define

$$
i\left(\epsilon_{1}, \epsilon_{2}\right)=\int_{a}^{b} g\left[x^{*}\right]_{\gamma}^{\alpha, \beta}(t) d t-C .
$$

The derivative $\frac{\partial i}{\partial \epsilon_{2}}$, when $\epsilon_{1}=\epsilon_{2}=0$, is

$$
\left.\frac{\partial i}{\partial \epsilon_{2}}\right|_{(0,0)}=\int_{a}^{b}\left(\partial_{2} g[x]_{\gamma}^{\alpha, \beta}(t) h_{2}(t)+\partial_{3} g[x]_{\gamma}^{\alpha, \beta}(t)^{C} D_{\gamma}^{\alpha(\cdot, \cdot), \beta(\cdot, \cdot)} h_{2}(t)\right) d t .
$$

Integrating by parts and choosing variations such that $h_{2}(b)=0$, we have

$$
\frac{\partial i}{\partial \epsilon_{2}} \mid(0,0)=\int_{a}^{b} h_{2}(t)\left[\partial_{2} g[x]_{\gamma}^{\alpha, \beta}(t)+D_{\bar{\gamma}, b}^{\beta(\cdot, \cdot), \alpha(\cdot, \cdot)} \partial_{3} g[x]_{\gamma}^{\alpha, \beta}(t)\right] d t .
$$

Thus, there exists a function $h_{2}$ such that

$$
\frac{\partial i}{\partial \epsilon_{2}} \mid(0,0) \neq 0
$$

We may apply the implicit function theorem to conclude that there exists a subfamily of variations satisfying the integral constraint. Consider the new function $j\left(\epsilon_{1}, \epsilon_{2}\right)=\mathcal{J}\left(x^{*}, T^{*}\right)$. Since $j$ has a local minimum at $(0,0)$ when subject to the constraint $i(\cdot, \cdot)=0$ and $\nabla i(0,0) \neq(0,0)$, there exists a number $\lambda$ such that

$$
\frac{\partial}{\partial \epsilon_{1}}(j-\lambda i)(0,0)=0 .
$$

Let $F=L-\lambda g$. Relation (4.55) can be written as 


$$
\begin{aligned}
& \int_{a}^{T} h_{1}(t)\left[\partial_{2} F[x]_{\gamma}^{\alpha, \beta}(t)+D_{\bar{\gamma}, T}^{\beta(\cdot, \cdot), \alpha(\cdot, \cdot)} \partial_{3} L[x]_{\gamma}^{\alpha, \beta}(t)-\lambda D_{\bar{\gamma}, b}^{\beta(\cdot, \cdot), \alpha(\cdot, \cdot)} \partial_{3} g[x]_{\gamma}^{\alpha, \beta}(t)\right] d t \\
& +\int_{T}^{b} h_{1}(t)\left[\gamma_{2}\left({ }_{a} D_{t}^{\beta(\cdot, \cdot)} \partial_{3} F[x]_{\gamma}^{\alpha, \beta}(t)-{ }_{T} D_{t}^{\beta(\cdot, \cdot)} \partial_{3} L[x]_{\gamma}^{\alpha, \beta}(t)\right)\right. \\
& \left.\quad-\lambda\left(\partial_{2} g[x]_{\gamma}^{\alpha, \beta}(t)+\gamma_{1 t} D_{b}^{\alpha(\cdot, \cdot)} \partial_{3} g[x]_{\gamma}^{\alpha, \beta}(t)\right)\right] d t \\
& +h_{1}(T)\left[\gamma_{1} I_{T}^{1-\alpha(\cdot, \cdot)} \partial_{3} L[x]_{\gamma}^{\alpha, \beta}(t)-\gamma_{2} I_{t}^{1-\beta(\cdot, \cdot)} \partial_{3} L[x]_{\gamma}^{\alpha, \beta}(t)+\partial_{2} \phi(t, x(t))\right]_{t=T} \\
& +\Delta T\left[L[x]_{\gamma}^{\alpha, \beta}(t)+\partial_{1} \phi(t, x(t))+\partial_{2} \phi(t, x(t)) x^{\prime}(t)\right]_{t=T} \\
& +h_{1}(b)\left[-\lambda \gamma_{1 t} I_{b}^{1-\alpha(\cdot, \cdot)} \partial_{3} g[x]_{\gamma}^{\alpha, \beta}(t)\right. \\
& \left.\quad+\gamma_{2}\left({ }_{T} I_{t}^{1-\beta(\cdot, \cdot)} \partial_{3} L[x]_{\gamma}^{\alpha, \beta}(t)-{ }_{a} I_{t}^{1-\beta(\cdot, \cdot)} \partial_{3} F[x]_{\gamma}^{\alpha, \beta}(t)\right)\right]_{t=b}=0 .
\end{aligned}
$$

Considering appropriate choices of variations, we obtain the first (4.52) and the second (4.53) necessary optimality conditions, and also the transversality conditions (4.54).

Similarly to Theorem [53, the following result holds.

Theorem 55 Suppose that $(x, T)$ gives a local minimum for functional (4.33) on $D$ subject to the boundary condition (4.51) and the isoperimetric constraint (4.50). Then there exists $\left(\lambda_{0}, \lambda\right) \neq(0,0)$ such that, if we define the function $F:[a, b] \times \mathbb{R}^{2} \rightarrow \mathbb{R}$ by $F=\lambda_{0} L-\lambda g,(x, T)$ satisfies the fractional EulerLagrange equations

$$
\partial_{2} F[x]_{\gamma}^{\alpha, \beta}(t)+D_{\bar{\gamma}, T}^{\beta(\cdot, \cdot), \alpha(\cdot, \cdot)} \partial_{3} L[x]_{\gamma}^{\alpha, \beta}(t)-\lambda D_{\bar{\gamma}, b}^{\beta(\cdot, \cdot), \alpha(\cdot, \cdot)} \partial_{3} g[x]_{\gamma}^{\alpha, \beta}(t)=0
$$

on the interval $[a, T]$, and

$$
\begin{aligned}
\gamma_{2}\left({ }_{a} D_{t}^{\beta(\cdot, \cdot)} \partial_{3} F[x]_{\gamma}^{\alpha, \beta}(t)-\right. & \left.{ }_{T} D_{t}^{\beta(\cdot, \cdot)} \partial_{3} L[x]_{\gamma}^{\alpha, \beta}(t)\right) \\
& -\lambda\left(\partial_{2} g[x]_{\gamma}^{\alpha, \beta}(t)+\gamma_{1 t} D_{b}^{\alpha(\cdot, \cdot)} \partial_{3} g[x]_{\gamma}^{\alpha, \beta}(t)\right)=0
\end{aligned}
$$

on the interval $[T, b]$.

\subsubsection{Example}

Let $\alpha(t, \tau)=\alpha(t)$ and $\beta(t, \tau)=\beta(\tau)$. Define the function

$$
\psi(T)=\int_{0}^{T}\left(\frac{t^{1-\alpha(t)}}{2 \Gamma(2-\alpha(t))}+\frac{(b-t)^{1-\beta(t)}}{2 \Gamma(2-\beta(t))}\right)^{2} d t
$$

on the interval $[0, b]$ with $b>0$. Consider the functional $J$ defined by 


$$
\begin{aligned}
J(x, t)=\int_{0}^{T}\left[\alpha(t)+\left({ }^{C} D_{\gamma}^{\alpha(\cdot, \cdot), \beta(\cdot, \cdot)} x(t)\right)^{2}\right. & \\
& \left.+\left(\frac{t^{1-\alpha(t)}}{2 \Gamma(2-\alpha(t))}+\frac{(b-t)^{1-\beta(t)}}{2 \Gamma(2-\beta(t))}\right)^{2}\right] d t
\end{aligned}
$$

for $t \in[0, b]$ and $\gamma=(1 / 2,1 / 2)$, subject to the initial condition

$$
x(0)=0
$$

and the isoperimetric constraint

$$
\int_{0}^{T}{ }^{C} D_{\gamma}^{\alpha(\cdot, \cdot), \beta(\cdot, \cdot)} x(t)\left(\frac{t^{1-\alpha(t)}}{2 \Gamma(2-\alpha(t))}+\frac{(b-t)^{1-\beta(t)}}{2 \Gamma(2-\beta(t))}\right)^{2} d t=\psi(T) .
$$

Define $F=L-\lambda g$ with $\lambda=2$, that is,

$$
F=\alpha(t)+\left({ }^{C} D_{\gamma}^{\alpha(\cdot), \beta(\cdot)} x(t)-\frac{t^{1-\alpha(t)}}{2 \Gamma(2-\alpha(t))}-\frac{(b-t)^{1-\beta(t)}}{2 \Gamma(2-\beta(t))}\right)^{2} .
$$

Consider the function $\bar{x}(t)=t$ with $t \in[0, b]$. Because

$$
{ }^{C} D_{\gamma}^{\alpha(\cdot, \cdot), \beta(\cdot, \cdot)} \bar{x}(t)=\frac{t^{1-\alpha(t)}}{2 \Gamma(2-\alpha(t))}+\frac{(b-t)^{1-\beta(t)}}{2 \Gamma(2-\beta(t))},
$$

we have that $\bar{x}$ satisfies conditions (4.39), (4.40) and the two last of (4.41). Using the first condition of (4.41), that is,

$$
\alpha(t)+2\left(\frac{T^{1-\alpha(T)}}{2 \Gamma(2-\alpha(T))}+\frac{(b-T)^{1-\beta(T)}}{2 \Gamma(2-\beta(T))}\right)^{2}=0,
$$

we obtain the optimal time $T$.

\subsection{Variational problems with holonomic constraints}

In this section, we present a new variational problem subject to a new type of constraints. A holonomic constraint is a condition of the form

$$
g(t, x)=0,
$$

where $x=\left(x_{1}, x_{2}, \ldots, x_{n}\right), n \geq 2$, and $g$ is a given function (see, e.g., (van Brunt, 2004)).

Consider the space

$$
U=\left\{\left(x_{1}, x_{2}, T\right) \in C^{1}([a, b]) \times C^{1}([a, b]) \times[a, b]: x_{1}(a)=x_{1 a} \wedge x_{2}(a)=x_{2 a}\right\}
$$

for fixed reals $x_{1 a}, x_{2 a} \in \mathbb{R}$. In this section, we consider the following variational problem: 
Problem 6 Find functions $x_{1}$ and $x_{2}$ that maximize or minimize the functional $\mathcal{J}$ defined in $U$ by

$$
\begin{aligned}
\mathcal{J}\left(x_{1}, x_{2}, T\right) & =\int_{a}^{T} L\left(t, x_{1}(t), x_{2}(t),{ }^{C} D_{\gamma}^{\alpha(\cdot, \cdot), \beta(\cdot, \cdot)} x_{1}(t),{ }^{C} D_{\gamma}^{\alpha(\cdot, \cdot), \beta(\cdot, \cdot)} x_{2}(t)\right) d t \\
& +\phi\left(T, x_{1}(T), x_{2}(T)\right)
\end{aligned}
$$

where the admissible functions satisfy the constraint

$$
g\left(t, x_{1}(t), x_{2}(t)\right)=0, \quad t \in[a, b],
$$

called a holonomic constraint, where $g:[a, b] \times \mathbb{R}^{2} \rightarrow \mathbb{R}$ is a continuous function and continuously differentiable with respect to second and third arguments.

The terminal time $T$ and terminal states $x_{1}(T)$ and $x_{2}(T)$ are free and the Lagrangian $L:[a, b] \times \mathbb{R}^{4} \rightarrow \mathbb{R}$ is a continuous function and continuously differentiable with respect to its ith argument, $i \in\{2,3,4,5\}$. The terminal cost function $\phi:[a, b] \times \mathbb{R}^{2} \rightarrow \mathbb{R}$ is of class $C^{1}$.

\subsubsection{Necessary optimality conditions}

The next theorem gives fractional necessary optimality conditions to the variational problem with a holonomic constraint. To simplify the notation, we denote by $x$ the vector $\left(x_{1}, x_{2}\right)$; by ${ }^{C} D_{\gamma}^{\alpha(\cdot, \cdot), \beta(\cdot, \cdot)} x$ we mean the two-dimensional vector $\left({ }^{C} D_{\gamma}^{\alpha(\cdot, \cdot), \beta(\cdot, \cdot)} x_{1},{ }^{C} D_{\gamma}^{\alpha(\cdot, \cdot), \beta(\cdot, \cdot)} x_{2}\right)$; and we use the operator

$$
[x]_{\gamma}^{\alpha, \beta}(t):=\left(t, x(t),{ }^{C} D_{\gamma}^{\alpha(\cdot, \cdot), \beta(\cdot, \cdot)} x(t)\right) .
$$

The next result given necessary optimality conditions for Problem 6

Theorem 56 Suppose that $(x, T)$ gives a local minimum to functional $\mathcal{J}$ as in (4.57), under the constraint (4.58) and the boundary conditions defined in (4.56). If

$$
\partial_{3} g(t, x(t)) \neq 0 \quad \forall t \in[a, b]
$$

then there exists a piecewise continuous function $\lambda:[a, b] \rightarrow \mathbb{R}$ such that $(x, T)$ satisfies the following fractional Euler-Lagrange equations:

$$
\partial_{2} L[x]_{\gamma}^{\alpha, \beta}(t)+D_{\bar{\gamma}, T}^{\beta(\cdot, \cdot), \alpha(\cdot, \cdot)} \partial_{4} L[x]_{\gamma}^{\alpha, \beta}(t)+\lambda(t) \partial_{2} g(t, x(t))=0
$$

and

$$
\partial_{3} L[x]_{\gamma}^{\alpha, \beta}(t)+D_{\bar{\gamma}, T}^{\beta(\cdot, \cdot), \alpha(\cdot, \cdot)} \partial_{5} L[x]_{\gamma}^{\alpha, \beta}(t)+\lambda(t) \partial_{3} g(t, x(t))=0
$$

on the interval $[a, T]$, and 


$$
\gamma_{2}\left({ }_{a} D_{t}^{\beta(\cdot, \cdot)} \partial_{4} L[x]_{\gamma}^{\alpha, \beta}(t)-{ }_{T} D_{t}^{\beta(\cdot, \cdot)} \partial_{4} L[x]_{\gamma}^{\alpha, \beta}(t)+\lambda(t) \partial_{2} g(t, x(t))\right)=0
$$

and

$$
{ }_{a} D_{t}^{\beta(\cdot, \cdot)} \partial_{5} L[x]_{\gamma}^{\alpha, \beta}(t)-{ }_{T} D_{t}^{\beta(\cdot, \cdot)} \partial_{5} L[x]_{\gamma}^{\alpha, \beta}(t)+\lambda(t) \partial_{3} g(t, x(t))=0
$$

on the interval $[T, b]$. Moreover, $(x, T)$ satisfies the transversality conditions

$$
\left\{\begin{array}{l}
L[x]_{\gamma}^{\alpha, \beta}(T)+\partial_{1} \phi(T, x(T))+\partial_{2} \phi(T, x(T)) x_{1}^{\prime}(T)+\partial_{3} \phi(T, x(T)) x_{2}^{\prime}(T)=0, \\
{\left[\gamma_{1 t} I_{T}^{1-\alpha(\cdot, \cdot)} \partial_{4} L[x]_{\gamma}^{\alpha, \beta}(t)-\gamma_{2} I_{t}^{1-\beta(\cdot, \cdot)} \partial_{4} L[x]_{\gamma}^{\alpha, \beta}(t)\right]_{t=T}+\partial_{2} \phi(T, x(T))=0,} \\
{\left[\gamma_{1 t} I_{T}^{1-\alpha(\cdot, \cdot)} \partial_{5} L[x]_{\gamma}^{\alpha, \beta}(t)-\gamma_{2 T} I_{t}^{1-\beta(\cdot, \cdot)} \partial_{5} L[x]_{\gamma}^{\alpha, \beta}(t)\right]_{t=T}+\partial_{3} \phi(T, x(T))=0,} \\
\gamma_{2}\left[{ }_{T} I_{t}^{1-\beta(\cdot, \cdot)} \partial_{4} L[x]_{\gamma}^{\alpha, \beta}(t)-{ }_{a} I_{t}^{1-\beta(\cdot, \cdot)} \partial_{4} L[x]_{\gamma}^{\alpha, \beta}(t)\right]_{t=b}=0 \\
\gamma_{2}\left[{ }_{T} I_{t}^{1-\beta(\cdot, \cdot)} \partial_{5} L[x]_{\gamma}^{\alpha, \beta}(t)-{ }_{a} I_{t}^{1-\beta(\cdot, \cdot)} \partial_{5} L[x]_{\gamma}^{\alpha, \beta}(t)\right]_{t=b}=0 .
\end{array}\right.
$$

Proof. Consider admissible variations of the optimal solution $(x, T)$ of the type

$$
\left(x^{*}, T^{*}\right)=(x+\epsilon h, T+\epsilon \Delta T),
$$

where $\epsilon \in \mathbb{R}$ is a small parameter, $h=\left(h_{1}, h_{2}\right) \in C^{1}([a, b]) \times C^{1}([a, b])$ satisfies $h_{i}(a)=0, i=1,2$, and $\triangle T \in \mathbb{R}$. Because

$$
\partial_{3} g(t, x(t)) \neq 0 \quad \forall t \in[a, b]
$$

by the implicit function theorem there exists a subfamily of variations of $(x, T)$ that satisfy (4.58), that is, there exists a unique function $h_{2}\left(\epsilon, h_{1}\right)$ such that the admissible variation $\left(x^{*}, T^{*}\right)$ satisfies the holonomic constraint (4.58):

$$
g\left(t, x_{1}(t)+\epsilon h_{1}(t), x_{2}(t)+\epsilon h_{2}(t)\right)=0 \quad \forall t \in[a, b] .
$$

Differentiating this condition with respect to $\epsilon$ and considering $\epsilon=0$, we obtain that

$$
\partial_{2} g(t, x(t)) h_{1}(t)+\partial_{3} g(t, x(t)) h_{2}(t)=0,
$$

which is equivalent to

$$
\frac{\partial_{2} g(t, x(t)) h_{1}(t)}{\partial_{3} g(t, x(t))}=-h_{2}(t)
$$

Define $j$ on a neighbourhood of zero by

$$
j(\epsilon)=\int_{a}^{T+\epsilon \triangle T} L\left[x^{*}\right]_{\gamma}^{\alpha, \beta}(t) d t+\phi\left(T+\epsilon \triangle T, x^{*}(T+\epsilon \triangle T)\right) .
$$

The derivative $\frac{\partial j}{\partial \epsilon}$ for $\epsilon=0$ is 


$$
\begin{aligned}
& \left.\frac{\partial j}{\partial \epsilon}\right|_{\epsilon=0}=\int_{a}^{T}\left(\partial_{2} L[x]_{\gamma}^{\alpha, \beta}(t) h_{1}(t)+\partial_{3} L[x]_{\gamma}^{\alpha, \beta}(t) h_{2}(t)\right. \\
& \left.+\partial_{4} L[x]_{\gamma}^{\alpha, \beta}(t)^{C} D_{\gamma}^{\alpha(\cdot, \cdot), \beta(\cdot, \cdot)} h_{1}(t)+\partial_{5} L[x]_{\gamma}^{\alpha, \beta}(t)^{C} D_{\gamma}^{\alpha(\cdot, \cdot), \beta(\cdot, \cdot)} h_{2}(t)\right) d t \\
& +L[x]_{\gamma}^{\alpha, \beta}(T) \triangle T+\partial_{1} \phi(T, x(T)) \triangle T+\partial_{2} \phi(T, x(T))\left[h_{1}(T)+x_{1}^{\prime}(T) \triangle T\right] \\
& +\partial_{3} \phi(T, x(T))\left[h_{2}(T)+x_{2}^{\prime}(T) \triangle T\right] .
\end{aligned}
$$

The third term in (4.65) can be written as

$$
\begin{aligned}
\int_{a}^{T} & \partial_{4} L[x]_{\gamma}^{\alpha, \beta}(t)^{C} D_{\gamma}^{\alpha(\cdot, \cdot), \beta(\cdot, \cdot)} h_{1}(t) d t \\
& =\int_{a}^{T} \partial_{4} L[x]_{\gamma}^{\alpha, \beta}(t)\left[\gamma_{1}{ }_{a}^{C} D_{t}^{\alpha(\cdot, \cdot)} h_{1}(t)+\gamma_{2}{ }_{t}^{C} D_{b}^{\beta(\cdot, \cdot)} h_{1}(t)\right] d t \\
& =\gamma_{1} \int_{a}^{T} \partial_{4} L[x]_{\gamma}^{\alpha, \beta}(t)_{a}^{C} D_{t}^{\alpha(\cdot, \cdot)} h_{1}(t) d t \\
& +\gamma_{2}\left[\int_{a}^{b} \partial_{4} L[x]_{\gamma}^{\alpha, \beta}(t)_{t}^{C} D_{b}^{\beta(\cdot, \cdot)} h_{1}(t) d t-\int_{T}^{b} \partial_{4} L[x]_{\gamma}^{\alpha, \beta}(t)_{t}^{C} D_{b}^{\beta(\cdot, \cdot)} h_{1}(t) d t\right] .
\end{aligned}
$$

Integrating by parts, (4.66) can be written as

$$
\begin{aligned}
\int_{a}^{T} h_{1}(t) & {\left[\gamma_{1 t} D_{T}^{\alpha(\cdot, \cdot)} \partial_{4} L[x]_{\gamma}^{\alpha, \beta}(t)+\gamma_{2 a} D_{t}^{\beta(\cdot, \cdot)} \partial_{4} L[x]_{\gamma}^{\alpha, \beta}(t)\right] d t } \\
+ & \int_{T}^{b} \gamma_{2} h_{1}(t)\left[{ }_{a} D_{t}^{\beta(\cdot, \cdot)} \partial_{4} L[x]_{\gamma}^{\alpha, \beta}(t)-{ }_{T} D_{t}^{\beta(\cdot, \cdot)} \partial_{4} L[x]_{\gamma}^{\alpha, \beta}(t)\right] d t \\
+ & {\left[h_{1}(t)\left(\gamma_{1 t} I_{T}{ }^{1-\alpha(\cdot, \cdot)} \partial_{4} L[x]_{\gamma}^{\alpha, \beta}(t)-\gamma_{2 T} I_{t}^{1-\beta(\cdot,)} \partial_{4} L[x]_{\gamma}^{\alpha, \beta}(t)\right)\right]_{t=T} } \\
+ & {\left[\gamma_{2} h_{1}(t)\left({ }_{T} I_{t}{ }^{1-\beta(\cdot, \cdot)} \partial_{4} L[x]_{\gamma}^{\alpha, \beta}(t)-{ }_{a} I_{t}{ }^{1-\beta(\cdot, \cdot)} \partial_{4} L[x]_{\gamma}^{\alpha, \beta}(t)\right)\right]_{t=b} . }
\end{aligned}
$$

By proceeding similarly to the 4 th term in (4.65), we obtain an equivalent expression. Substituting these relations into (4.65) and considering the fractional operator $D_{\bar{\gamma}, c}^{\beta(\cdot, \cdot), \alpha(\cdot, \cdot)}$ as defined in (4.34), we obtain that 


$$
\begin{aligned}
& 0=\int_{a}^{T}\left[h_{1}(t)\left[\partial_{2} L[x]_{\gamma}^{\alpha, \beta}(t)+D_{\bar{\gamma}, T}^{\beta(\cdot, \cdot), \alpha(\cdot, \cdot)} \partial_{4} L[x]_{\gamma}^{\alpha, \beta}(t)\right]\right. \\
& \left.+h_{2}(t)\left[\partial_{3} L[x]_{\gamma}^{\alpha, \beta}(t)+D_{\bar{\gamma}, T}^{\beta(\cdot, \cdot), \alpha(\cdot, \cdot)} \partial_{5} L[x]_{\gamma}^{\alpha, \beta}(t)\right]\right] d t \\
& +\gamma_{2} \int_{T}^{b}\left[h_{1}(t)\left[{ }_{a} D_{t}^{\beta(\cdot, \cdot)} \partial_{4} L[x]_{\gamma}^{\alpha, \beta}(t)-{ }_{T} D_{t}^{\beta(\cdot, \cdot)} \partial_{4} L[x]_{\gamma}^{\alpha, \beta}(t)\right]\right. \\
& \left.+h_{2}(t)\left[{ }_{a} D_{t}^{\beta(\cdot, \cdot)} \partial_{5} L[x]_{\gamma}^{\alpha, \beta}(t)-{ }_{T} D_{t}^{\beta(\cdot, \cdot)} \partial_{5} L[x]_{\gamma}^{\alpha, \beta}(t)\right] d t\right] \\
& +h_{1}(T)\left[\gamma_{1 t} I_{T}{ }^{1-\alpha(\cdot, \cdot)} \partial_{4} L[x]_{\gamma}^{\alpha, \beta}(t)\right. \\
& \left.-\gamma_{2 T} I_{t}{ }^{1-\beta(\cdot, \cdot)} \partial_{4} L[x]_{\gamma}^{\alpha, \beta}(t)+\partial_{2} \phi(t, x(t))\right]_{t=T} \\
& +h_{2}(T)\left[\gamma_{1 t} I_{T}^{1-\alpha(\cdot, \cdot)} \partial_{5} L[x]_{\gamma}^{\alpha, \beta}(t)-\gamma_{2 T} I_{t}^{1-\beta(\cdot, \cdot)} \partial_{5} L[x]_{\gamma}^{\alpha, \beta}(t)+\partial_{3} \phi(t, x(t))\right]_{t=T} \\
& +\triangle T\left[L[x]_{\gamma}^{\alpha, \beta}(t)+\partial_{1} \phi(t, x(t))+\partial_{2} \phi(t, x(t)) x_{1}^{\prime}(t)+\partial_{3} \phi(t, x(t)) x_{2}^{\prime}(t)\right]_{t=T} \\
& +h_{1}(b)\left[\gamma_{2}\left({ }_{T} I_{t}^{1-\beta(\cdot, \cdot)} \partial_{4} L[x]_{\gamma}^{\alpha, \beta}(t)-{ }_{a} I_{t}^{1-\beta(\cdot, \cdot)} \partial_{4} L[x]_{\gamma}^{\alpha, \beta}(t)\right)\right]_{t=b} \\
& +h_{2}(b)\left[\gamma_{2}\left({ }_{T} I_{t}{ }^{1-\beta(\cdot, \cdot)} \partial_{5} L[x]_{\gamma}^{\alpha, \beta}(t)-{ }_{a} I_{t}^{1-\beta(\cdot, \cdot)} \partial_{5} L[x]_{\gamma}^{\alpha, \beta}(t)\right)\right]_{t=b} .
\end{aligned}
$$

Define the piecewise continuous function $\lambda$ by

$$
\lambda(t)= \begin{cases}-\frac{\partial_{3} L[x]_{\gamma}^{\alpha, \beta}(t)+D_{\bar{\gamma}, T}^{\beta(\cdot, \cdot), \alpha(\cdot, \cdot)} \partial_{5} L[x]_{\gamma}^{\alpha, \beta}(t)}{\partial_{3} g(t, x(t))}, & t \in[a, T] \\ -\frac{{ }_{a} D_{t}^{\beta(\cdot, \cdot)} \partial_{5} L[x]_{\gamma}^{\alpha, \beta}(t)-T D_{t}^{\beta(\cdot, \cdot)} \partial_{5} L[x]_{\gamma}^{\alpha, \beta}(t)}{\partial_{3} g(t, x(t))}, & t \in[T, b] .\end{cases}
$$

Using equations (4.64) and (4.68), we obtain that

$$
\begin{aligned}
\lambda(t) \partial_{2} g & t, x(t)) h_{1}(t) \\
\quad= & \begin{cases}\left(\partial_{3} L[x]_{\gamma}^{\alpha, \beta}(t)+D_{\bar{\gamma}, T}^{\beta(\cdot, \cdot), \alpha(\cdot, \cdot)} \partial_{5} L[x]_{\gamma}^{\alpha, \beta}(t)\right) h_{2}(t), & t \in[a, T] \\
\left({ }_{a} D_{t}^{\beta(\cdot, \cdot)} \partial_{5} L[x]_{\gamma}^{\alpha, \beta}(t)-{ }_{T} D_{t}^{\beta(\cdot, \cdot)} \partial_{5} L[x]_{\gamma}^{\alpha, \beta}(t)\right) h_{2}(t), & t \in[T, b] .\end{cases}
\end{aligned}
$$

Substituting in (4.67), we have 


$$
\begin{aligned}
& 0=\int_{a}^{T} h_{1}(t)\left[\partial_{2} L[x]_{\gamma}^{\alpha, \beta}(t)+D_{\bar{\gamma}, T}^{\beta(\cdot, \cdot), \alpha(\cdot, \cdot)} \partial_{4} L[x]_{\gamma}^{\alpha, \beta}(t)+\lambda(t) \partial_{2} g(t, x(t))\right] d t \\
& +\gamma_{2} \int_{T}^{b} h_{1}(t)\left[{ }_{a} D_{t}^{\beta(\cdot, \cdot)} \partial_{4} L[x]_{\gamma}^{\alpha, \beta}(t)-{ }_{T} D_{t}^{\beta(\cdot, \cdot)} \partial_{4} L[x]_{\gamma}^{\alpha, \beta}(t)+\lambda(t) \partial_{2} g(t, x(t))\right] d t \\
& +h_{1}(T)\left[\gamma_{1 t} I_{T}{ }^{1-\alpha(\cdot, \cdot)} \partial_{4} L[x]_{\gamma}^{\alpha, \beta}(t)-\gamma_{2 T} I_{t}^{1-\beta(\cdot, \cdot)} \partial_{4} L[x]_{\gamma}^{\alpha, \beta}(t)+\partial_{2} \phi(t, x(t))\right]_{t=T} \\
& +h_{2}(T)\left[\gamma_{1 t} I_{T}{ }^{1-\alpha(\cdot, \cdot)} \partial_{5} L[x]_{\gamma}^{\alpha, \beta}(t)-\gamma_{2 T} I_{t}^{1-\beta(\cdot, \cdot)} \partial_{5} L[x]_{\gamma}^{\alpha, \beta}(t)+\partial_{3} \phi(t, x(t))\right]_{t=T} \\
& +\triangle T\left[L[x]_{\gamma}^{\alpha, \beta}(t)+\partial_{1} \phi(t, x(t))+\partial_{2} \phi(t, x(t)) x_{1}^{\prime}(t)+\partial_{3} \phi(t, x(t)) x_{2}^{\prime}(t)\right]_{t=T} \\
& +h_{1}(b)\left[\gamma_{2}\left({ }_{T} I_{t}{ }^{1-\beta(\cdot, \cdot)} \partial_{4} L[x]_{\gamma}^{\alpha, \beta}(t)-{ }_{a} I_{t}^{1-\beta(\cdot, \cdot)} \partial_{4} L[x]_{\gamma}^{\alpha, \beta}(t)\right)\right]_{t=b} \\
& +h_{2}(b)\left[\gamma_{2}\left({ }_{T} I_{t}{ }^{1-\beta(\cdot, \cdot)} \partial_{5} L[x]_{\gamma}^{\alpha, \beta}(t)-{ }_{a} I_{t}^{1-\beta(\cdot, \cdot)} \partial_{5} L[x]_{\gamma}^{\alpha, \beta}(t)\right)\right]_{t=b} .
\end{aligned}
$$

Considering appropriate choices of variations, we obtained the first (4.59) and the third (4.61) necessary conditions, and also the transversality conditions (4.63). The remaining conditions (4.60) and (4.62) follow directly from (4.68).

\subsubsection{Example}

We end this section with a simple illustrative example. Consider the following problem:

$$
\begin{gathered}
J(x, t)=\int_{0}^{T}\left[\alpha(t)+\left({ }^{C} D_{\gamma}^{\alpha(\cdot, \cdot), \beta(\cdot, \cdot)} x_{1}(t)-\frac{t^{1-\alpha(t)}}{2 \Gamma(2-\alpha(t))}-\frac{(b-t)^{1-\beta(t)}}{2 \Gamma(2-\beta(t))}\right)^{2}\right. \\
\left.+\left({ }^{C} D_{\gamma}^{\alpha(\cdot, \cdot), \beta(\cdot, \cdot)} x_{2}(t)\right)^{2}\right] d t \longrightarrow \min , \\
x_{1}(t)+x_{2}(t)=t+1, \\
x_{1}(0)=0, \quad x_{2}(0)=1 .
\end{gathered}
$$

It is a simple exercise to check that $x_{1}(t)=t, x_{2}(t) \equiv 1$ and $\lambda(t) \equiv 0$ satisfy our Theorem 56.

\subsection{Fractional variational Herglotz problem}

In this section, we study fractional variational problems of Herglotz type, depending on the combined Caputo fractional derivatives ${ }^{C} D_{\gamma}^{\alpha(\cdot, \cdot), \beta(\cdot, \cdot)}$. Two 
different cases are considered.

The variational problem of Herglotz is a generalization of the classical variational problem. It allows us to describe nonconservative processes, even in case the Lagrange function is autonomous (that is, when the Lagrangian does not depend explicitly on time). In opposite to calculus of variations, where the cost functional is given by an integral depending only on time, space and on the dynamics, in the Herglotz variational problem the model is given by a differential equation involving the derivative of the objective function $z$, and the Lagrange function depends on time, trajectories $x$ and $z$, and on the derivative of $x$. The problem of Herglotz was posed by (Herglotz, 1930), but only in 1996, with the works (Guenther, Gottsch and Kramer, 1996; Guenther, Guenther and Gottsch, 1996), it has gained the attention of the mathematical community. Since then, several papers were devoted to this subject. For example, see references (Almeida and Malinowska, 2014; Georgieva and Guenther, 2002; Georgieva. Guenther and Bodurov, 2003; Santos, Martins and Torres, 2014, 2015a, b).

In Section 4.7.1, we obtain fractional Euler-Lagrange conditions for the fractional variational problem of Herglotz, with one variable, and the general case, for several independent variables is discussed in Section 4.7.2. Finally, three illustrative examples are presented in detail (Section 4.7.3).

\subsubsection{Fundamental problem of Herglotz}

Let $\alpha, \beta:[a, b]^{2} \rightarrow(0,1)$ be two functions. The fractional Herglotz variational problem that we study is as follows.

Problem 7 Determine the trajectories $x \in C^{1}([a, b] ; \mathbb{R})$ satisfying a given boundary condition $x(a)=x_{a}$, for a fixed $x_{a} \in \mathbb{R}$, and a real $T \in(a, b]$, that extremize the value of $z(T)$, where $z$ satisfies the differential equation

$$
\dot{z}(x, t)=L\left(t, x(t),{ }^{C} D_{\gamma}^{\alpha(\cdot, \cdot), \beta(\cdot, \cdot)} x(t), z(t)\right), \quad t \in[a, b],
$$

with dependence on a combined Caputo fractional derivative operator, subject to the initial condition

$$
z(a)=z_{a}
$$

where $z_{a}$ is a fixed real number.

In the sequel, we use the auxiliary notation

$$
[x, z]_{\gamma}^{\alpha, \beta}(t)=\left(t, x(t),{ }^{C} D_{\gamma}^{\alpha(\cdot, \cdot), \beta(\cdot, \cdot)} x(t), z(t)\right) .
$$

The Lagrangian $L$ is assumed to satisfy the following hypothesis:

1. $L:[a, b] \times \mathbb{R}^{3} \rightarrow \mathbb{R}$ is differentiable, 
2. $t \rightarrow \lambda(t) \partial_{3} L[x, z]_{\gamma}^{\alpha, \beta}(t)$ is such that ${ }_{T} D_{t}^{\beta(\cdot, \cdot)}\left(\lambda(t) \partial_{3} L[x, z]_{\gamma}^{\alpha, \beta}(t)\right)$, ${ }_{a} D_{t}^{\beta(\cdot, \cdot)}\left(\lambda(t) \partial_{3} L[x, z]_{\gamma}^{\alpha, \beta}(t)\right)$, and $D_{\bar{\gamma}}^{\beta(\cdot, \cdot), \alpha(\cdot, \cdot)}\left(\lambda(t) \partial_{3} L[x, z]_{\gamma}^{\alpha, \beta}(t)\right)$ exist and are continuous on $[a, b]$, where

$$
\lambda(t)=\exp \left(-\int_{a}^{t} \partial_{4} L[x, z]_{\gamma}^{\alpha, \beta}(\tau) d \tau\right) .
$$

The following result gives necessary conditions of Euler-Lagrange type for a solution of Problem 7

Theorem 57 Let $x \in C^{1}([a, b] ; \mathbb{R})$ be such that $z$ defined by Eq. (4.69), subject to the initial condition (4.70), has an extremum at $T \in] a, b]$. Then, $(x, z)$ satisfies the fractional differential equations

$$
\partial_{2} L[x, z]_{\gamma}^{\alpha, \beta}(t) \lambda(t)+D_{\bar{\gamma}}^{\beta(\cdot, \cdot), \alpha(\cdot, \cdot)}\left(\lambda(t) \partial_{3} L[x, z]_{\gamma}^{\alpha, \beta}(t)\right)=0,
$$

on $[a, T]$, and

$$
\gamma_{2}\left({ }_{a} D_{t}^{\beta(\cdot, \cdot)}\left(\lambda(t) \partial_{3} L[x, z]_{\gamma}^{\alpha, \beta}(t)\right)-{ }_{T} D_{t}^{\beta(\cdot, \cdot)}\left(\lambda(t) \partial_{3} L[x, z]_{\gamma}^{\alpha, \beta}(t)\right)\right)=0,
$$

on $[T, b]$. Moreover, the following transversality conditions are satisfied:

$$
\left\{\begin{array}{l}
{\left[\gamma_{1 t} I_{T}^{1-\alpha(\cdot, \cdot)}\left(\lambda(t) \partial_{3} L[x, z]_{\gamma}^{\alpha, \beta}(t)\right)-\gamma_{2 T} I_{t}^{1-\beta(\cdot, \cdot)}\left(\lambda(t) \partial_{3} L[x, z]_{\gamma}^{\alpha, \beta}(t)\right)\right]_{t=T}=0,} \\
\gamma_{2}\left[{ }_{T} I_{t}^{1-\beta(\cdot, \cdot)}\left(\lambda(t) \partial_{3} L[x, z]_{\gamma}^{\alpha, \beta}(t)\right)-{ }_{a} I_{t}^{1-\beta(\cdot, \cdot)}\left(\lambda(t) \partial_{3} L[x, z]_{\gamma}^{\alpha, \beta}(t)\right)\right]_{t=b}=0 .
\end{array}\right.
$$

If $T<b$, then $L[x, z]_{\gamma}^{\alpha, \beta}(T)=0$.

Proof. Let $x$ be a solution of the problem. Consider an admissible variation of $x, \bar{x}=x+\epsilon h$, where $h \in C^{1}([a, b] ; \mathbb{R})$ is an arbitrary perturbation curve and $\epsilon \in \mathbb{R}$ represents a small number $(|\epsilon| \ll 1)$. The constraint $x(a)=x_{a}$ implies that all admissible variations must fulfill the condition $h(a)=0$. On the other hand, consider an admissible variation of $z, \bar{z}=z+\epsilon \theta$, where $\theta$ is a perturbation curve (not arbitrary) such that

1. $\theta(a)=0$, so that $z(a)=z_{a}$,

2. $\theta(T)=0$ because $z(T)$ is a maximum (or a minimum), i.e., $\bar{z}(T)-z(T) \leq 0 \quad(\bar{z}(T)-z(T) \geq 0)$,

3. $\theta(t)=\left.\frac{d}{d \varepsilon} z(\bar{x}, t)\right|_{\varepsilon=0}$, so that the variation satisfies the differential equation (4.69).

Differentiating $\theta$ with respect to $t$, we obtain that

$$
\begin{aligned}
\frac{d}{d t} \theta(t) & =\left.\frac{d}{d t} \frac{d}{d \varepsilon} z(\bar{x}, t)\right|_{\varepsilon=0} \\
& =\left.\frac{d}{d \varepsilon} \frac{d}{d t} z(\bar{x}, t)\right|_{\varepsilon=0} \\
& =\left.\frac{d}{d \varepsilon} L\left(t, x(t)+\epsilon h(t),{ }^{C} D_{\gamma}^{\alpha(\cdot, \cdot), \beta(\cdot, \cdot)} x(t)+\epsilon^{C} D_{\gamma}^{\alpha(\cdot, \cdot), \beta(\cdot, \cdot)} h(t), \bar{z}(\bar{x}, t)\right)\right|_{\varepsilon=0},
\end{aligned}
$$


and rewriting this relation, we obtain the following differential equation for $\theta$ : $\dot{\theta}(t)-\partial_{4} L[x, z]_{\gamma}^{\alpha, \beta}(t) \theta(t)=\partial_{2} L[x, z]_{\gamma}^{\alpha, \beta}(t) h(t)+\partial_{3} L[x, z]_{\gamma}^{\alpha, \beta}(t)^{C} D_{\gamma}^{\alpha(\cdot, \cdot), \beta(\cdot, \cdot)} h(t)$.

Considering $\lambda(t)=\exp \left(-\int_{a}^{t} \partial_{4} L[x, z]_{\gamma}^{\alpha, \beta}(\tau) d \tau\right)$, we obtain the solution for the last differential equation

$$
\begin{aligned}
\theta(T) & \lambda(T)-\theta(a) \\
= & \int_{a}^{T}\left(\partial_{2} L[x, z]_{\gamma}^{\alpha, \beta}(t) h(t)+\partial_{3} L[x, z]_{\gamma}^{\alpha, \beta}(t)^{C} D_{\gamma}^{\alpha(\cdot, \cdot), \beta(\cdot, \cdot)} h(t)\right) \lambda(t) d t .
\end{aligned}
$$

By hypothesis, $\theta(a)=0$. If $x$ is such that $z(x, t)$ defined by (4.69) attains an extremum at $t=T$, then $\theta(T)$ is identically zero. Hence, we get

$$
\int_{a}^{T}\left(\partial_{2} L[x, z]_{\gamma}^{\alpha, \beta}(t) h(t)+\partial_{3} L[x, z]_{\gamma}^{\alpha, \beta}(t)^{C} D_{\gamma}^{\alpha(\cdot, \cdot), \beta(\cdot, \cdot)} h(t)\right) \lambda(t) d t=0 .
$$

Considering only the second term in Eq. (4.74) and the definition of combined Caputo derivative operator, we obtain that

$$
\begin{gathered}
\int_{a}^{T} \lambda(t) \partial_{3} L[x, z]_{\gamma}^{\alpha, \beta}(t)\left(\gamma_{1}^{C} D_{t}^{\alpha(\cdot, \cdot)} h(t)+\gamma_{2 t}^{C} D_{b}^{\beta(\cdot, \cdot)} h(t)\right) d t \\
=\gamma_{1} \int_{a}^{T} \lambda(t) \partial_{3} L[x, z]_{\gamma}^{\alpha, \beta}(t)_{a}^{C} D_{t}^{\alpha(\cdot, \cdot)} h(t) d t \\
+\gamma_{2}\left[\int_{a}^{b} \lambda(t) \partial_{3} L[x, z]_{\gamma}^{\alpha, \beta}(t)_{t}^{C} D_{b}^{\beta(\cdot, \cdot)} h(t) d t\right. \\
\left.\quad-\int_{T}^{b} \lambda(t) \partial_{3} L[x, z]_{\gamma}^{\alpha, \beta}(t)_{t}^{C} D_{b}^{\beta(\cdot, \cdot)} h(t) d t\right]=\star .
\end{gathered}
$$

Using the fractional integration by parts formula and considering $\bar{\gamma}=\left(\gamma_{2}, \gamma_{1}\right)$, we get

$$
\begin{aligned}
& \star \\
& \star \int_{a}^{T} h(t) D_{\bar{\gamma}}^{\beta(\cdot, \cdot), \alpha(\cdot, \cdot)}\left(\lambda(t) \partial_{3} L[x, z]_{\gamma}^{\alpha, \beta}(t)\right) d t \\
& +\int_{T}^{b} \gamma_{2} h(t)\left[{ }_{a} D_{t}^{\beta(\cdot, \cdot)}\left(\lambda(t) \partial_{3} L[x, z]_{\gamma}^{\alpha, \beta}(t)\right)-{ }_{T} D_{t}^{\beta(\cdot, \cdot)}\left(\lambda(t) \partial_{3} L[x, z]_{\gamma}^{\alpha, \beta}(t)\right)\right] d t \\
& +h(T)\left[\gamma_{1 t} I_{T}^{1-\alpha(\cdot, \cdot)}\left(\lambda(t) \partial_{3} L[x, z]_{\gamma}^{\alpha, \beta}(t)\right)-\gamma_{2 T} I_{t}^{1-\beta(\cdot, \cdot)}\left(\lambda(t) \partial_{3} L[x, z]_{\gamma}^{\alpha, \beta}(t)\right)\right]_{t=T} \\
& +h(b) \gamma_{2}\left[{ }_{T} I_{t}^{1-\beta(\cdot, \cdot)}\left(\lambda(t) \partial_{3} L[x, z]_{\gamma}^{\alpha, \beta}(t)\right)-{ }_{a} I_{t}^{1-\beta(\cdot, \cdot)}\left(\lambda(t) \partial_{3} L[x, z]_{\gamma}^{\alpha, \beta}(t)\right)\right]_{t=b} .
\end{aligned}
$$

Substituting this relation into expression (4.74), we obtain 


$$
\begin{aligned}
& 0=\int_{a}^{T} h(t)\left[\partial_{2} L[x, z]_{\gamma}^{\alpha, \beta}(t) \lambda(t)+D_{\gamma}^{\beta(\cdot, \cdot), \alpha(\cdot, \cdot)}\left(\lambda(t) \partial_{3} L[x, z]_{\gamma}^{\alpha, \beta}(t)\right)\right] d t \\
& +\int_{T}^{b} \gamma_{2} h(t)\left[{ }_{a} D_{t}^{\beta(\cdot, \cdot)}\left(\lambda(t) \partial_{3} L[x, z]_{\gamma}^{\alpha, \beta}(t)\right)-{ }_{T} D_{t}^{\beta(\cdot, \cdot)}\left(\lambda(t) \partial_{3} L[x, z]_{\gamma}^{\alpha, \beta}(t)\right)\right] d t \\
& +h(T)\left[\gamma_{1 t} I_{T}^{1-\alpha(\cdot, \cdot)}\left(\lambda(t) \partial_{3} L[x, z]_{\gamma}^{\alpha, \beta}(t)\right)-\gamma_{2 T} I_{t}^{1-\beta(\cdot, \cdot)}\left(\lambda(t) \partial_{3} L[x, z]_{\gamma}^{\alpha, \beta}(t)\right)\right]_{t=T} \\
& +h(b) \gamma_{2}\left[{ }_{T} I_{t}^{1-\beta(\cdot, \cdot)}\left(\lambda(t) \partial_{3} L[x, z]_{\gamma}^{\alpha, \beta}(t)\right)-{ }_{a} I_{t}^{1-\beta(\cdot, \cdot)}\left(\lambda(t) \partial_{3} L[x, z]_{\gamma}^{\alpha, \beta}(t)\right)\right]_{t=b} .
\end{aligned}
$$

With appropriate choices for the variations $h(\cdot)$, we get the Euler-Lagrange equations (4.71)-(4.72) and the transversality conditions (4.73).

Remark 58 If $\alpha(\cdot, \cdot)$ and $\beta(\cdot, \cdot)$ tend to 1 , and if the Lagrangian $L$ is of class $C^{2}$, then the first Euler-Lagrange equation (4.71) becomes

$$
\partial_{2} L[x, z]_{\gamma}^{\alpha, \beta}(t) \lambda(t)+\left(\gamma_{2}-\gamma_{1}\right) \frac{d}{d t}\left[\lambda(t) \partial_{3} L[x, z]_{\gamma}^{\alpha, \beta}(t)\right]=0 .
$$

Differentiating and considering the derivative of the lambda function, we obtain

$$
\begin{aligned}
& \lambda(t)\left[\partial_{2} L[x, z]_{\gamma}^{\alpha, \beta}(t)\right. \\
& \left.\quad+\left(\gamma_{2}-\gamma_{1}\right)\left[-\partial_{4} L[x, z]_{\gamma}^{\alpha, \beta}(t) \partial_{3} L[x, z]_{\gamma}^{\alpha, \beta}(t)+\frac{d}{d t} \partial_{3} L[x, z]_{\gamma}^{\alpha, \beta}(t)\right]\right]=0 .
\end{aligned}
$$

As $\lambda(t)>0$, for all $t$, we deduce that

$$
\partial_{2} L[x, z]_{\gamma}^{\alpha, \beta}(t)+\left(\gamma_{2}-\gamma_{1}\right)\left[\frac{d}{d t} \partial_{3} L[x, z]_{\gamma}^{\alpha, \beta}(t)-\partial_{4} L[x, z]_{\gamma}^{\alpha, \beta}(t) \partial_{3} L[x, z]_{\gamma}^{\alpha, \beta}(t)\right]=0 .
$$

\subsubsection{Several independent variables}

Consider the following generalization of the problem of Herglotz involving $n+1$ independent variables. Define $\Omega=\prod_{i=1}^{n}\left[a_{i}, b_{i}\right]$, with $n \in \mathbb{N}, P=[a, b] \times \Omega$ and consider the vector $s=\left(s_{1}, s_{2}, \ldots, s_{n}\right) \in \Omega$. The new problem consists in determining the trajectories $x \in C^{1}(P)$ that give an extremum to $z[x, T]$, where the functional $z$ satisfies the differential equation

$$
\begin{aligned}
\dot{z}(t)= & \int_{\Omega} L\left(t, s, x(t, s),{ }^{C} D_{\gamma}^{\alpha(\cdot, \cdot), \beta(\cdot, \cdot)} x(t, s),\right. \\
& \left.{ }^{C} D_{\gamma^{1}}^{\alpha_{1}(\cdot, \cdot), \beta_{1}(\cdot, \cdot)} x(t, s), \ldots,{ }^{C} D_{\gamma^{n}}^{\alpha_{n}(\cdot, \cdot), \beta_{n}(\cdot, \cdot)} x(t, s), z(t)\right) d^{n} s
\end{aligned}
$$

subject to the constraint

$$
x(t, s)=g(t, s), \quad \text { for all } \quad(t, s) \in \partial P,
$$

where $\partial P$ is the boundary of $P$ and $g$ is a given function $g: \partial P \rightarrow \mathbb{R}$. We assume that 
1. $\alpha, \alpha_{i}, \beta, \beta_{i}:[a, b]^{2} \rightarrow(0,1)$ with $i=1, \ldots, n$,

2. $\gamma, \gamma^{1}, \ldots, \gamma^{n} \in[0,1]^{2}$,

3. $d^{n} s=d s_{1} \ldots d s_{n}$

4. ${ }^{C} D_{\gamma}^{\alpha(\cdot, \cdot), \beta(\cdot, \cdot)} x(t, s),{ }^{C} D_{\gamma^{1}}^{\alpha_{1}(\cdot, \cdot), \beta_{1}(\cdot, \cdot)} x(t, s), \ldots,{ }^{C} D_{\gamma^{n}}^{\alpha_{n}(\cdot, \cdot), \beta_{n}(\cdot, \cdot)} x(t, s)$ exist and are continuous functions,

5. the Lagrangian $L: P \times \mathbb{R}^{n+3} \rightarrow \mathbb{R}$ is of class $C^{1}$.

Remark $59 B y^{C} D_{\gamma}^{\alpha(\cdot, \cdot), \beta(\cdot, \cdot)} x(t, s)$ we mean the Caputo fractional derivative with respect to the independent variable $t$, and by ${ }^{C} D_{\gamma^{i}}^{\alpha_{i}(\cdot, \cdot), \beta_{i}(\cdot, \cdot)} x(t, s)$ we mean the Caputo fractional derivative with respect to the independent variable $s_{i}$, for $i=1, \ldots, n$.

In the sequel, we use the auxiliary notation $[x, z]_{n, \gamma}^{\alpha, \beta}(t, s)$ to represent the following vector

$$
\begin{aligned}
\left(t, s, x(t, s),{ }^{C} D_{\gamma}^{\alpha(\cdot, \cdot), \beta(\cdot, \cdot)} x(t, s),{ }^{C} D_{\gamma^{1}}^{\alpha_{1}(\cdot, \cdot), \beta_{1}(\cdot, \cdot)} x(t, s),\right. \\
\left.\ldots,{ }^{C} D_{\gamma^{n}}^{\alpha_{n}(\cdot, \cdot), \beta_{n}(\cdot, \cdot)} x(t, s), z(t)\right) .
\end{aligned}
$$

Consider the function

$$
\lambda(t)=\exp \left(-\int_{a}^{t} \int_{\Omega} \partial_{2 n+4}[x, z]_{n, \gamma}^{\alpha, \beta}(\tau, s) d^{n} s d \tau\right) .
$$

Theorem 60 If $(x, z, T)$ is an extremizer of the functional defined by Eq. (4.75), then $(x, z, T)$ satisfies the fractional differential equations

$$
\begin{aligned}
& \partial_{n+2} L[x, z]_{n, \gamma}^{\alpha, \beta}(t, s) \lambda(t)+D_{\bar{\gamma}}^{\beta(\cdot, \cdot), \alpha(\cdot, \cdot)}\left(\lambda(t) \partial_{n+3} L[x, z]_{n, \gamma}^{\alpha, \beta}(t, s)\right) \\
&+\sum_{i=1}^{n} D_{\bar{\gamma}^{i}}^{\beta_{i}(\cdot, \cdot), \alpha_{i}(\cdot, \cdot)}\left(\lambda(t) \partial_{n+3+i} L[x, z]_{n, \gamma}^{\alpha, \beta}(t, s)\right)=0
\end{aligned}
$$

on $[a, T] \times \Omega$, and

$\gamma_{2}\left({ }_{a} D_{t}^{\beta(\cdot, \cdot)}\left(\lambda(t) \partial_{n+3} L[x, z]_{n, \gamma}^{\alpha, \beta}(t, s)\right)-{ }_{T} D_{t}^{\beta(\cdot, \cdot)}\left(\lambda(t) \partial_{n+3} L[x, z]_{n, \gamma}^{\alpha, \beta}(t, s)\right)\right)=0$

on $[T, b] \times \Omega$.

Moreover, $(x, z)$ satisfies the transversality condition

$$
\begin{aligned}
& {\left[\gamma_{1 t} I_{T}^{1-\alpha(\cdot, \cdot)}\left(\lambda(t) \partial_{n+3} L[x, z]_{n, \gamma}^{\alpha, \beta}(t, s)\right)\right.} \\
& \left.\quad-\gamma_{2 T} I_{t}^{1-\beta(\cdot, \cdot)}\left(\lambda(t) \partial_{n+3} L[x, z]_{n, \gamma}^{\alpha, \beta}(t, s)\right)\right]_{t=T}=0, \quad s \in \Omega
\end{aligned}
$$

If $T<b$, then $\int_{\Omega} L[x, z]_{n, \gamma}^{\alpha, \beta}(T, s) d^{n} s=0$. 
Proof. Let $x$ be a solution of the problem. Consider an admissible variation of $x, \bar{x}(t, s)=x(t, s)+\epsilon h(t, s)$, where $h \in C^{1}(P)$ is an arbitrary perturbing curve and $\epsilon \in \mathbb{R}$ is such that $|\epsilon| \ll 1$. Consequently, $h(t, s)=0$ for all $(t, s) \in \partial P$ by the boundary condition (4.76).

On the other hand, consider an admissible variation of $z, \bar{z}=z+\epsilon \theta$, where $\theta$ is a perturbing curve such that $\theta(a)=0$ and

$$
\theta(t)=\left.\frac{d}{d \varepsilon} z(\bar{x}, t)\right|_{\varepsilon=0} .
$$

Differentiating $\theta(t)$ with respect to $t$, we obtain that

$$
\begin{aligned}
\frac{d}{d t} \theta(t) & =\left.\frac{d}{d t} \frac{d}{d \varepsilon} z(\bar{x}, t)\right|_{\varepsilon=0} \\
& =\left.\frac{d}{d \varepsilon} \frac{d}{d t} z(\bar{x}, t)\right|_{\varepsilon=0} \\
& =\left.\frac{d}{d \varepsilon} \int_{\Omega} L[\bar{x}, z]_{n, \gamma}^{\alpha, \beta}(t, s) d^{n} s\right|_{\varepsilon=0} .
\end{aligned}
$$

We conclude that

$$
\begin{gathered}
\dot{\theta}(t)=\int_{\Omega}\left(\partial_{n+2} L[x, z]_{n, \gamma}^{\alpha, \beta}(t, s) h(t, s)+\partial_{n+3} L[x, z]_{n, \gamma}^{\alpha, \beta}(t, s)^{C} D_{\gamma}^{\alpha(\cdot, \cdot), \beta(\cdot, \cdot)} h(t, s)\right. \\
\left.+\sum_{i=1}^{n} \partial_{n+3+i} L[x, z]_{n, \gamma}^{\alpha, \beta}(t, s)^{C} D_{\gamma^{i}}^{\alpha_{i}(\cdot, \cdot), \beta_{i}(\cdot, \cdot)} h(t, s)+\partial_{2 n+4} L[x, z]_{n, \gamma}^{\alpha, \beta}(t, s) \theta(t)\right) d^{n} s .
\end{gathered}
$$

To simplify the notation, we define

$$
B(t)=\int_{\Omega} \partial_{2 n+4} L[x, z]_{n, \gamma}^{\alpha, \beta}(t, s) d^{n} s
$$

and

$$
\begin{aligned}
A(t)=\int_{\Omega}\left(\partial_{n+2} L[x, z]_{n, \gamma}^{\alpha, \beta}(t, s) h(t, s)+\partial_{n+3} L[x, z]_{n, \gamma}^{\alpha, \beta}(t, s)^{C} D_{\gamma}^{\alpha(\cdot, \cdot), \beta(\cdot, \cdot)} h(t, s)\right. \\
\left.+\sum_{i=1}^{n} \partial_{n+3+i} L[x, z]_{n, \gamma}^{\alpha, \beta}(t, s)^{C} D_{\gamma^{i}}^{\alpha_{i}(\cdot, \cdot), \beta_{i}(\cdot, \cdot)} h(t, s)\right) d^{n} s .
\end{aligned}
$$

Then, we obtain the linear differential equation

$$
\dot{\theta}(t)-B(t) \theta(t)=A(t),
$$

whose solution is

$$
\theta(T) \lambda(T)-\theta(a)=\int_{a}^{T} A(t) \lambda(t) d t .
$$


Since $\theta(a)=\theta(T)=0$, we get

$$
\int_{a}^{T} A(t) \lambda(t) d t=0 .
$$

Considering only the second term in (4.80), we can write

$$
\begin{gathered}
\int_{a}^{T} \int_{\Omega} \lambda(t) \partial_{n+3} L[x, z]_{n, \gamma}^{\alpha, \beta}(t, s)\left(\gamma_{1 a}^{C} D_{t}^{\alpha(\cdot, \cdot)} h(t, s)+\gamma_{2}{ }_{t}^{C} D_{b}^{\beta(\cdot, \cdot)} h(t, s)\right) d^{n} s d t \\
=\gamma_{1} \int_{a}^{T} \int_{\Omega} \lambda(t) \partial_{n+3} L[x, z]_{n, \gamma}^{\alpha, \beta}(t, s)_{a}^{C} D_{t}^{\alpha(\cdot, \cdot)} h(t, s) d^{n} s d t \\
+\gamma_{2}\left[\int_{a}^{b} \int_{\Omega} \lambda(t) \partial_{n+3} L[x, z]_{n, \gamma}^{\alpha, \beta}(t, s)_{t}^{C} D_{b}^{\beta(\cdot, \cdot)} h(t, s) d^{n} s d t\right. \\
\left.\quad-\int_{T}^{b} \int_{\Omega} \lambda(t) \partial_{n+3} L[x, z]_{n, \gamma}^{\alpha, \beta}(t, s)_{t}^{C} D_{b}^{\beta(\cdot, \cdot)} h(t, s) d^{n} s d t\right] .
\end{gathered}
$$

Let $\bar{\gamma}=\left(\gamma_{2}, \gamma_{1}\right)$. Integrating by parts (cf. Theorem 13), and since $h(a, s)=$ $h(b, s)=0$ for all $s \in \Omega$, we obtain the following expression:

$$
\begin{gathered}
\int_{a}^{T} \int_{\Omega} h(t, s) D_{\bar{\gamma}}^{\beta(\cdot, \cdot), \alpha(\cdot, \cdot)}\left(\lambda(t) \partial_{n+3} L[x, z]_{n, \gamma}^{\alpha, \beta}(t, s)\right) d^{n} s d t \\
+\gamma_{2} \int_{T}^{b} \int_{\Omega} h(t, s)\left[{ }_{a} D_{t}^{\beta(\cdot, \cdot)}\left(\lambda(t) \partial_{n+3} L[x, z]_{n, \gamma}^{\alpha, \beta}(t, s)\right)\right. \\
\left.-{ }_{T} D_{t}^{\beta(\cdot, \cdot)}\left(\lambda(t) \partial_{n+3} L[x, z]_{n, \gamma}^{\alpha, \beta}(t, s)\right)\right] d^{n} s d t \\
+\int_{\Omega} h(T, s)\left[\gamma_{1 t} I_{T}^{1-\alpha(\cdot, \cdot)}\left(\lambda(t) \partial_{n+3} L[x, z]_{n, \gamma}^{\alpha, \beta}(t, s)\right)\right. \\
\left.-\gamma_{2} I_{t}^{1-\beta(\cdot, \cdot)}\left(\lambda(t) \partial_{n+3} L[x, z]_{n, \gamma}^{\alpha, \beta}(t, s)\right) d^{n} s\right]_{t=T}
\end{gathered} .
$$

Doing similarly for the $(i+2)$ th term in (4.80), with $i=1, \ldots, n$, letting $\bar{\gamma}^{i}=\left(\gamma_{2}^{i}, \gamma_{1}^{i}\right)$, and since $h\left(t, a_{i}\right)=h\left(t, b_{i}\right)=0$ for all $t \in[a, b]$, we obtain

$$
\begin{gathered}
\int_{a}^{T} \int_{\Omega} \lambda(t) \partial_{n+3+i} L[x, z]_{n, \gamma}^{\alpha, \beta}(t, s)\left(\gamma_{1 a_{i}}^{i C} D_{s_{i}}^{\alpha_{i}(\cdot, \cdot)} h(t, s)+\gamma_{2 s_{i}}^{i C} D_{b_{i}}^{\beta_{i}(\cdot, \cdot)} h(t, s)\right) d^{n} s d t \\
=\int_{a}^{T} \int_{\Omega} h(t, s) D_{\bar{\gamma}^{i}}^{\beta_{i}(\cdot, \cdot), \alpha_{i}(\cdot, \cdot)}\left(\lambda(t) \partial_{n+3+i} L[x, z]_{n, \gamma}^{\alpha, \beta}(t, s)\right) d^{n} s d t .
\end{gathered}
$$

Substituting these relations into (4.80), we deduce that 


$$
\begin{gathered}
\int_{a}^{T} \int_{\Omega} h(t, s)\left[\partial_{n+2} L[x, z]_{n, \gamma}^{\alpha, \beta}(t, s) \lambda(t)+D_{\bar{\gamma}}^{\beta(\cdot, \cdot), \alpha(\cdot, \cdot)}\left(\lambda(t) \partial_{n+3} L[x, z]_{n, \gamma}^{\alpha, \beta}(t, s)\right)\right. \\
+\sum_{i=1}^{n} D_{\bar{\gamma}^{i}}^{\beta_{i}(\cdot, \cdot), \alpha_{i}(\cdot, \cdot)}\left(\lambda(t) \partial_{n+3+i} L[x, z]_{n, \gamma}^{\alpha, \beta}(t, s)\right) d^{n} s d t \\
+\gamma_{2} \int_{T}^{b} \int_{\Omega} h(t, s)\left[{ }_{a} D_{t}^{\beta(\cdot, \cdot)}\left(\lambda(t) \partial_{n+3} L[x, z]_{n, \gamma}^{\alpha, \beta}(t, s)\right)\right. \\
\left.-{ }_{T} D_{t}^{\beta(\cdot, \cdot)}\left(\lambda(t) \partial_{n+3} L[x, z]_{n, \gamma}^{\alpha, \beta}(t, s)\right)\right] d^{n} s d t \\
+\int_{\Omega} h(T, s)\left[\gamma_{1 t} I_{T}^{1-\alpha(\cdot, \cdot)}\left(\lambda(t) \partial_{n+3} L[x, z]_{n, \gamma}^{\alpha, \beta}(t, s)\right)\right. \\
\left.-\gamma_{2} T_{t}^{1-\beta(\cdot, \cdot)}\left(\lambda(t) \partial_{n+3} L[x, z]_{n, \gamma}^{\alpha, \beta}(t, s)\right) d^{n} s\right]_{t=T} .
\end{gathered}
$$

For appropriate choices with respect to $h$, we get the Euler-Lagrange equations (4.77) -(4.78) and the transversality condition (4.79).

\subsubsection{Examples}

We present three examples, with and without the dependence on $z$.

Example 4.1. Consider

$$
\begin{gathered}
\dot{z}(t)=\left({ }^{C} D_{\gamma}^{\alpha(\cdot, \cdot), \beta(\cdot, \cdot)} x(t)\right)^{2}+z(t)+t^{2}-1, \quad t \in[0,3], \\
x(0)=1, \quad z(0)=0 .
\end{gathered}
$$

In this case, $\lambda(t)=\exp (-t)$. The necessary optimality conditions (4.71) (4.72) of Theorem 57 hold for $\bar{x}(t) \equiv 1$. If we replace $x$ by $\bar{x}$ in (4.81), we obtain

$$
\begin{gathered}
\dot{z}(t)-z(t)=t^{2}-1, \quad t \in[0,3], \\
z(0)=0,
\end{gathered}
$$

whose solution is

$$
z(t)=\exp (t)-(t+1)^{2} .
$$

The last transversality condition of Theorem 57 asserts that

$$
L[\bar{x}, z]_{\gamma}^{\alpha, \beta}(T)=0 \Leftrightarrow \exp (T)-2 T-2=0,
$$

whose solution is approximately

$$
T \approx 1.67835 .
$$

We remark that $z$ (4.82) actually attains a minimum value at this point (see Figure 4.1, (a)):

$$
z(1.67835) \approx-1.81685
$$


Example 4.2. Consider now

$$
\begin{gathered}
\dot{z}(t)=(t-1)\left(x^{2}(t)+z^{2}(t)+1\right), \quad t \in[0,3], \\
x(0)=0, \quad z(0)=0 .
\end{gathered}
$$

Since the first Euler-Lagrange equation (4.71) reads

$$
(t-1) x(t)=0, \quad \forall t \in[0, T],
$$

we see that $\bar{x}(t) \equiv 0$ is a solution of this equation. The second transversality condition of (4.73) asserts that, at $t=T$, we must have

$$
L[\bar{x}, z]_{\gamma}^{\alpha, \beta}(t)=0
$$

that is,

$$
(t-1)\left(z^{2}(t)+1\right)=0,
$$

and so $T=1$ is a solution for this equation. Substituting $x$ by $\bar{x}$ in (4.83), we get

$$
\begin{gathered}
\dot{z}(t)=(t-1)\left(z^{2}(t)+1\right), \quad t \in[0,3], \\
z(0)=0 .
\end{gathered}
$$

The solution to this Cauchy problem is the function

$$
z(t)=\tan \left(\frac{t^{2}}{2}-t\right)
$$

(see Figure 4.1. (b)) and the minimum value is

$$
z(1)=\tan \left(-\frac{1}{2}\right) .
$$

Example 4.3. For our last example, consider

$$
\begin{gathered}
\dot{z}(t)=\left({ }^{C} D_{\gamma}^{\alpha(\cdot, \cdot), \beta(\cdot, \cdot)} x(t)-f(t)\right)^{2}+t^{2}-1, \quad t \in[0,3] \\
x(0)=0, \quad z(0)=0
\end{gathered}
$$

where

$$
f(t):=\frac{t^{1-\alpha(t)}}{2 \Gamma(2-\alpha(t))}-\frac{(3-t)^{1-\beta(t)}}{2 \Gamma(2-\beta(t))} .
$$

In this case, $\lambda(t) \equiv 1$. We intend to find a pair $(x, z)$, satisfying all the conditions in (4.84), for which $z(T)$ attains a minimum value. It is easy to verify that $\bar{x}(t)=t$ and $T=1$ satisfy the necessary conditions given by Theorem 57. Replacing $x$ by $\bar{x}$ in (4.84), we get a Cauchy problem of form

$$
\begin{gathered}
\dot{z}(t)=t^{2}-1, \quad t \in[0,3], \\
z(0)=0,
\end{gathered}
$$


whose solution is

$$
z(t)=\frac{t^{3}}{3}-t
$$

Observe that this function attains a minimum value at $T=1$, which is $z(1)=$ $-2 / 3$ (Figure 4.1. (c)).

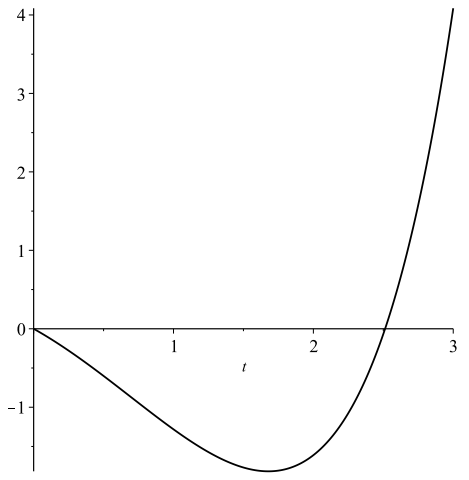

(a) Extremal $z$ of Example 4.1

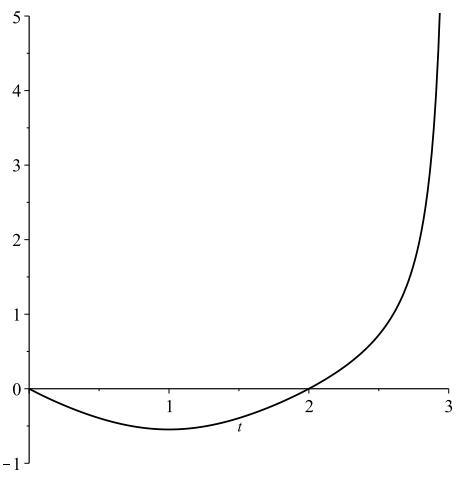

(b) Extremal $z$ of Example 4.2

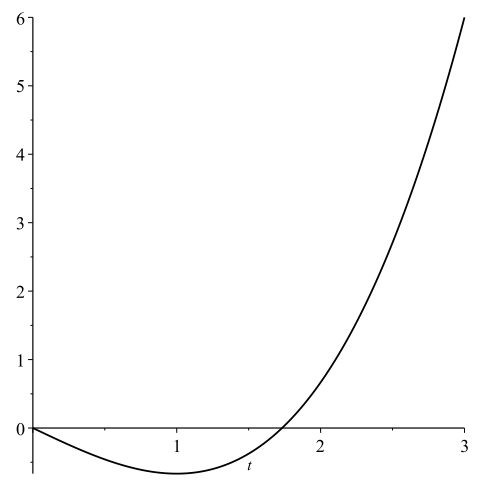

(c) Extremal $z$ of Example 4.3

Fig. 4.1. Graphics of function $z(\bar{x}, t)$.

\section{References}

Almeida R (2016) Fractional variational problems depending on indefinite integrals and with delay. Bull. Malays. Math. Sci. Soc. 39(4):1515-1528 arXiv: 1512.06752 
Almeida R, Malinowska AB (2014) Fractional variational principle of Herglotz. Discrete Contin. Dyn. Syst. Ser. B 19(8):2367-2381

Baleanu D, Maaraba T, Jarad F (2008) Fractional variational principles with delay. J. Phys. A 41(31):315403, 8 pp

Caputo MC, Torres DFM (2015) Duality for the left and right fractional derivatives. Signal Process. 107:265-271 arXiv:1409.5319

Daftardar-Gejji V, Sukale Y, Bhalekar S (2015) Solving fractional delay differential equations: A new approach. Fract. Calc. Appl. Anal. 16:400-418

Deng W, Li C, Lü J (2007) Stability analysis of linear fractional differential system with multiple time delays. Nonlinear Dynam. 48(4):409-416

Georgieva B, Guenther RB (2002) First Noether-type theorem for the generalized variational principle of Herglotz. Topol. Methods Nonlinear Anal. 20(2):261-273

Georgieva B, Guenther RB, Bodurov T (2003) Generalized variational principle of Herglotz for several independent variables. J. Math. Phys. 44(9):39113927

Guenther RB, Gottsch JA, Kramer DB (1996) The Herglotz algorithm for constructing canonical transformations. SIAM Rev. 38(2):287-293

Guenther RB, Guenther CM, Gottsch JA (1996) The Herglotz Lectures on Contact Transformations and Hamiltonian Systems. Lecture Notes in Nonlinear Analysis, Vol. 1, Juliusz Schauder Center for Nonlinear Studies, Nicholas Copernicus University, Torún

Herglotz G (1930) Berührungstransformationen, Lectures at the University of Göttingen, Göttingen

Jarad F, Abdeljawad T, Baleanu D (2010) Fractional variational principles with delay within Caputo derivatives. Rep. Math. Phys. 65(1):17-28

Lazarević MP, Spasić AM (2009) Finite-time stability analysis of fractional order time-delay systems: Gronwall's approach. Math. Comput. Model. 49(34):475-481

Linge S, Langtangen HP (2016) Programming for ComputationsMATLAB/Octave. A Gentle Introduction to Numerical Simulations with MATLAB/Octave. Springer, Cham

Machado JAT (2011) Time-delay and fractional derivatives. Adv. Difference Equ. Vol 2011:934094, 12 pp

Malinowska AB, Odzijewicz T, Torres DFM (2015) Advanced Methods in the Fractional Calculus of Variations. Springer Briefs in Applied Sciences and Technology, Springer, Cham

Santos SPS, Martins N, Torres DFM (2014) Higher-order variational problems of Herglotz type. Vietnam J. Math. 42(4):409-419 arXiv: 1309.6518

Santos SPS, Martins N, Torres DFM (2015a) Variational problems of Herglotz type with time delay: DuBois-Reymond condition and Noether's first theorem. Discrete Contin. Dyn. Syst. 35(9):4593-4610 arXiv: 1501.04873

Santos SPS, Martins N, Torres DFM (2015b) An optimal control approach to Herglotz variational problems. In Optimization in the Natural Sciences (eds. 
A. Plakhov, T. Tchemisova and A. Freitas), Communications in Computer and Information Science, Vol. 499, Springer:107-117 arXiv:1412.0433

Tavares D, Almeida R, Torres DFM (2015) Optimality conditions for fractional variational problems with dependence on a combined Caputo derivative of variable order. Optimization 64(6):1381-1391 arXiv:1501.02082

Tavares D, Almeida R, Torres DFM (2017) Constrained fractional variational problems of variable order. IEEE/CAA Journal Automatica Sinica 4(1):8088 arXiv: 1606.07512

Tavares D, Almeida R, Torres DFM (2018a) Fractional Herglotz variational problem of variable order. Discrete Contin. Dyn. Syst. Ser. S 11(1):143-154. arXiv: 1703.09104

Tavares D, Almeida R, Torres DFM (2018b) Combined fractional variational problems of variable order and some computational aspects. J. Comput. Appl. Math. 339:374-388. arXiv:1704.06486

Trefethen LN (2013) Approximation Theory and Approximation Practice. Society for Industrial and Applied Mathematics

van Brunt B (2004) The Calculus of Variations. Universitext, Springer, New York

Wang H, Yu Y, Wen G, Zhang S (2015) Stability analysis of fractional order neural networks with time delay. Neural Process. Lett. 42(2):479-500 


\section{Appendix}

In this appendix, we use a specific MATLAB software, the package Chebfun, to obtain a few computational approximations for the main fractional operators in this book.

Chebfun is an open source software package that "aims to provide numerical computing with functions" in MATLAB (Linge and Langtangen, 2016). Chebfun overloads MATLAB's discrete operations for matrices to analogous continuous operations for functions and operators (Trefethen, 2013). For the mathematical underpinnings of Chebfun, we refer the reader to (Trefethen, 2013). For the algorithmic backstory of Chebfun, we refer to (Driscoll, Hale and Trefethen, 2014).

In what follows, we study some computational approximations of RiemannLiouville fractional integrals, of Caputo fractional derivatives and consequently of the combined Caputo fractional derivative, all of them with variable-order. We provide, also, the necessary Chebfun code for the variableorder fractional calculus.

To implement these operators, we need two auxiliary functions: the gamma function $\Gamma$ (Definition (1) and the beta function $B$ (Definition 3). Both functions are available in MATLAB through the commands gamma(t) and $\operatorname{beta}(t, u)$, respectively.

\section{A.1 Higher-order Riemann-Liouville fractional integrals}

In this section, we discuss computational aspects to the higher-order RiemannLiouville fractional integrals of variable-order ${ }_{a} I_{t}^{\alpha_{n}(\cdot, \cdot)} x(t)$ and ${ }_{t} I_{b}^{\alpha_{n}(\cdot, \cdot)} x(t)$.

Considering the Definition 34 of higher-order Riemann-Liouville fractional integrals, we implemented in Chebfun two functions leftFi ( $x, a l p h a, a)$ and rightFI ( $x$, alpha , $b$ ) that approximate, respectively, the Riemann-Liouville fractional integrals ${ }_{a} I_{t}^{\alpha_{n}(\cdot, \cdot)} x(t)$ and ${ }_{t} I_{b}^{\alpha_{n}(\cdot, \cdot)} x(t)$, through the following Chebfun/MATLAB code. 


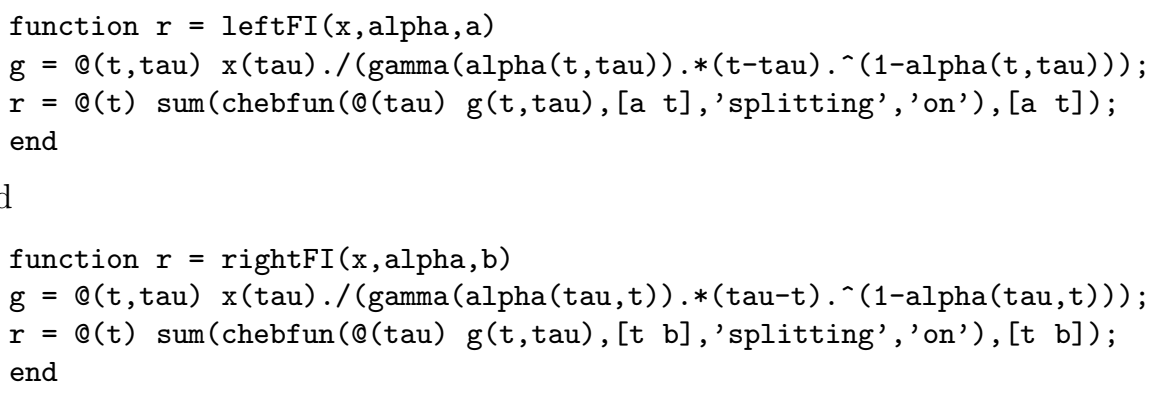

With these two functions, we illustrate their use in the following example, where we determine computacional approximations for Riemann-Liouville fractional integrals of a special power function.

Example 4.4. Let $\alpha(t, \tau)=\frac{t^{2}+\tau^{2}}{4}$ and $x(t)=t^{2}$ with $t \in[0,1]$. In this case, $a=0, b=1$ and $n=1$. We have ${ }_{a} I_{0.6}^{\alpha(\cdot, \cdot)} x(0.6) \approx 0.2661$ and ${ }_{0.6} I_{b}^{\alpha(\cdot, \cdot)} x(0.6) \approx$ 0.4619 , obtained in MATLAB with our Chebfun functions as follows:

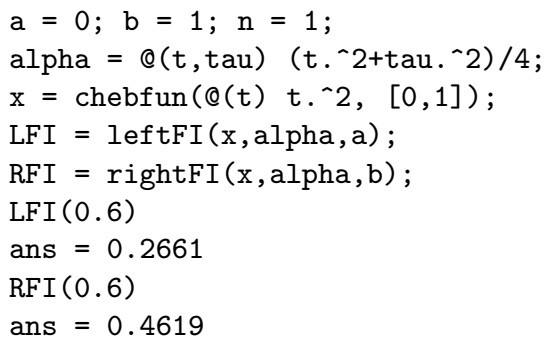

Other values for ${ }_{a} I_{t}^{\alpha(\cdot, \cdot)} x(t)$ and ${ }_{t} I_{b}^{\alpha(\cdot, \cdot)} x(t)$ are plotted in Figure 4.2 ,

\section{A.2 Higher-order Caputo fractional derivatives}

In this section, considering the Definition [36] we implement in Chebfun two new functions leftCaputo ( $x, a l p h a, a, n)$ and rightCaputo $(x, a l p h a, b, n)$ that approximate, respectively, the higher-order Caputo fractional derivatives of variable-order ${ }_{a}^{C} D_{t}^{\alpha_{n}(\cdot, \cdot)} x(t)$ and ${ }_{t}^{C} D_{b}^{\alpha_{n}(\cdot, \cdot)} x(t)$.

The following code implements the left operator (4.2):

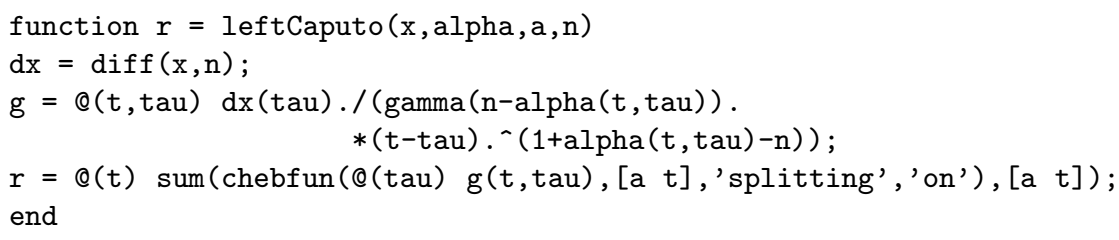

Similarly, we define the right operator (4.3) with Chebfun in MATLAB as follows: 


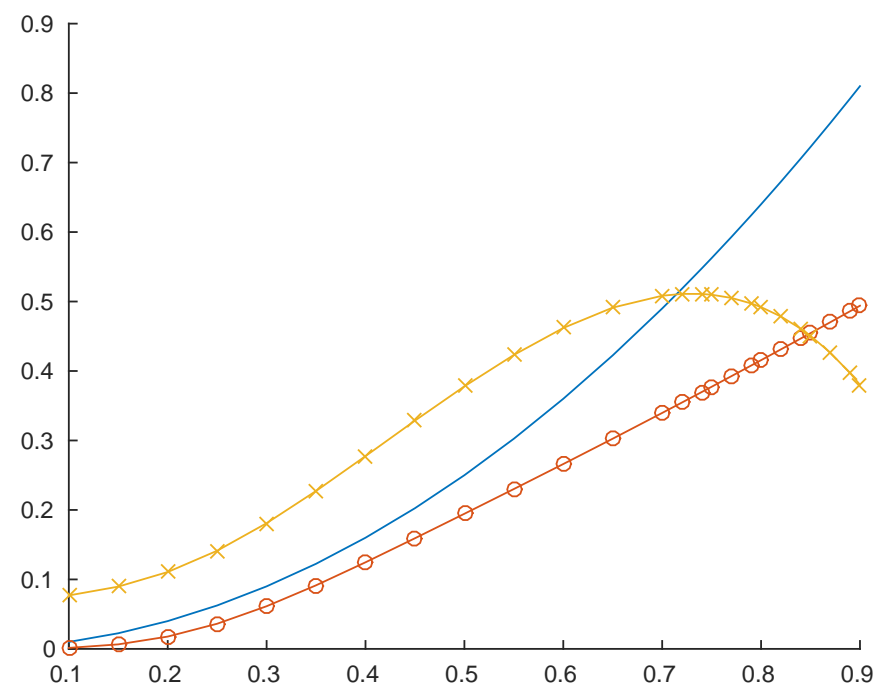

Fig. 4.2. Riemann-Liouville fractional integrals of Example 4.4 $x(t)=t^{2}$ in continuous line, left integral ${ }_{a} I_{t}^{\alpha(\cdot, \cdot)} x(t)$ with "o-" style, and right integral ${ }_{t} I_{b}^{\alpha(\cdot, \cdot)} x(t)$ with " $\times-$ " style.

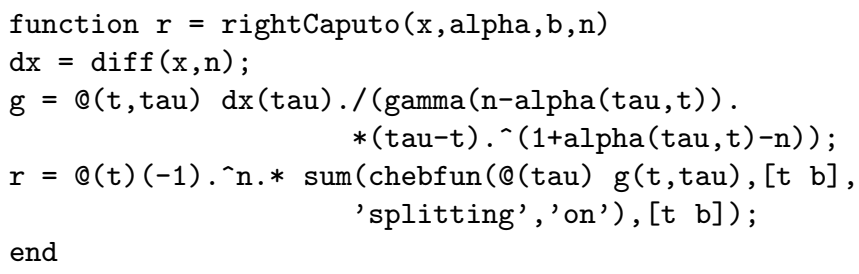

end

We use the two functions leftCaputo and rightCaputo to determine aproximations for the Caputo fractional derivatives of a power function of the form $x(t)=t^{\gamma}$.

Example 4.5. Let $\alpha(t, \tau)=\frac{t^{2}}{2}$ and $x(t)=t^{4}$ with $t \in[0,1]$. In this case, $a=0$, $b=1$ and $n=1$. We have ${ }_{a}^{C} D_{0.6}^{\alpha(\cdot, \cdot)} x(0.6) \approx 0.1857$ and ${ }_{0.6}^{C} D_{b}^{\alpha(\cdot, \cdot)} x(0.6) \approx$ -1.0385 , obtained in MATLAB with our Chebfun functions as follows:

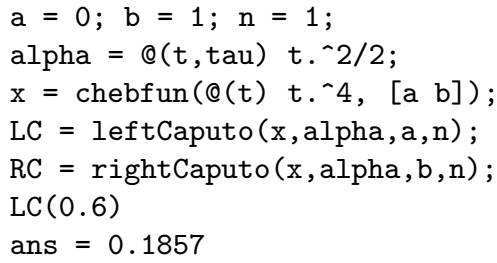


ans $=-1.0385$

See Figure 4.3 for a plot with other values of ${ }_{a}^{C} D_{t}^{\alpha(\cdot, \cdot)} x(t)$ and ${ }_{t}^{C} D_{b}^{\alpha(\cdot, \cdot)} x(t)$.

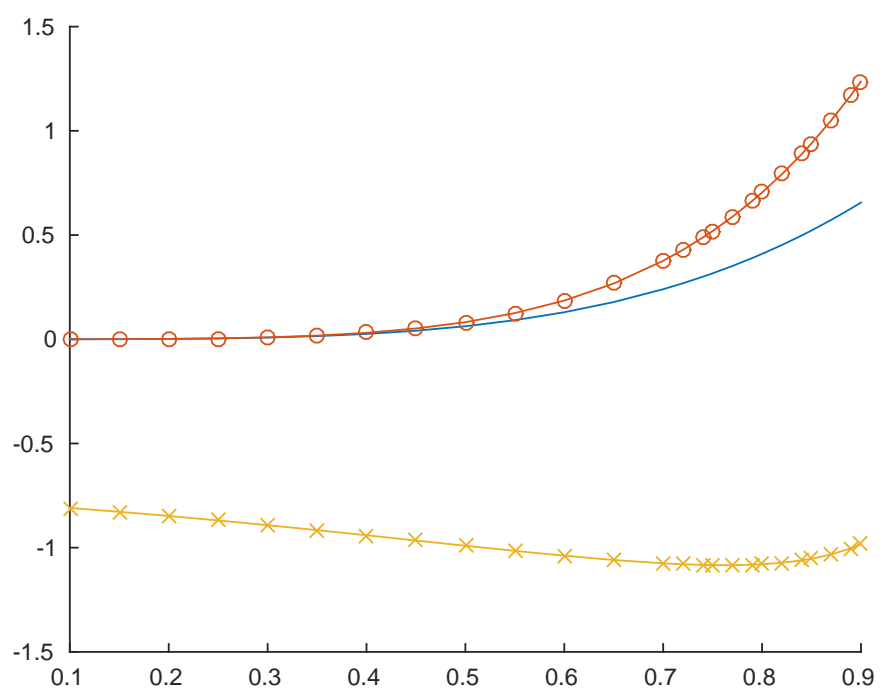

Fig. 4.3. Caputo fractional derivatives of Example 4.5 $x(t)=t^{4}$ in continuous line, left derivative ${ }_{a}^{C} D_{t}^{\alpha(\cdot, \cdot)} x(t)$ with "o-" style, and right derivative ${ }_{t}^{C} D_{b}^{\alpha(\cdot, \cdot)} x(t)$ with " $\times-"$ style.

Example 4.6. In Example 4.5, we have used the polynomial $x(t)=t^{4}$. It is worth mentioning that our Chebfun implementation works well for functions that are not a polynomial. For example, let $x(t)=e^{t}$. In this case, we just need to change

$\mathrm{x}=\operatorname{chebfun}(@(t)$ t.^4,[a b $]) ;$

in Example 4.5 by

$x=\operatorname{chebfun}(@(t) \exp (t),[a b]) ;$

to obtain

$\mathrm{LC}(0.6)$

ans $=0.9917$

$\mathrm{RC}(0.6)$

ans $=-1.1398$

See Figure 4.4 for a plot with other values of ${ }_{a}^{C} D_{t}^{\alpha(\cdot, \cdot)} x(t)$ and ${ }_{t}^{C} D_{b}^{\alpha(\cdot, \cdot)} x(t)$. 


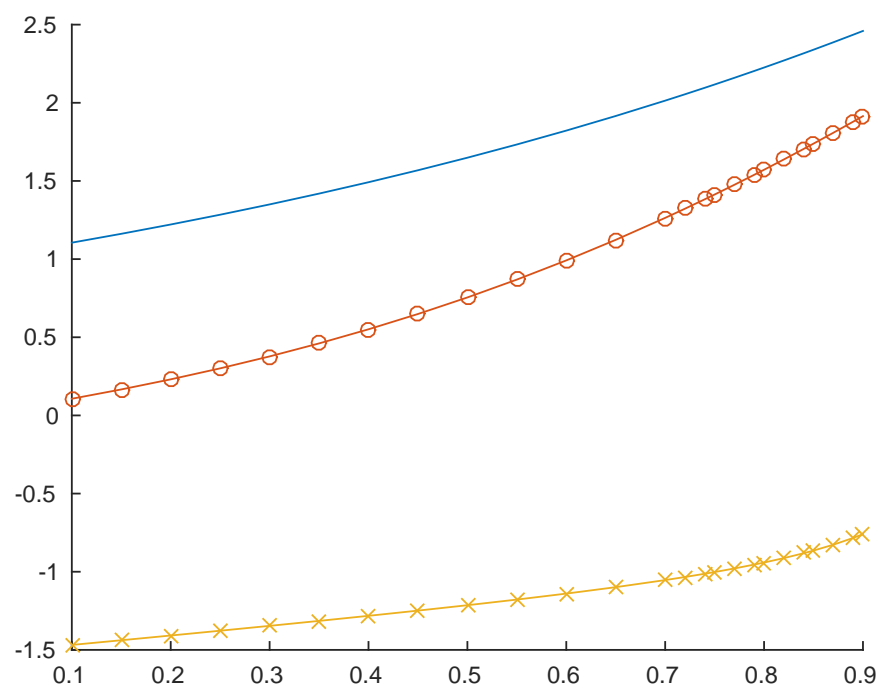

Fig. 4.4. Caputo fractional derivatives of Example 4.6 $x(t)=e^{t}$ in continuous line, left derivative ${ }_{a}^{C} D_{t}^{\alpha(\cdot, \cdot)} x(t)$ with "o-" style, and right derivative ${ }_{t}^{C} D_{b}^{\alpha(\cdot, \cdot)} x(t)$ with " $\times$-" style.

With Lemma 40 in Section 4.1 we can obtain, analytically, the higher-order left Caputo fractional derivative of a power function of the form $x(t)=(t-a)^{\gamma}$. This allows us to show the effectiveness of our computational approach, that is, the usefulness of polynomial interpolation in Chebyshev points in fractional calculus of variable-order. In Lemma 40, we assume that the fractional order depends only on the first variable: $\alpha_{n}(t, \tau):=\bar{\alpha}_{n}(t)$, where $\bar{\alpha}_{n}:[a, b] \rightarrow$ $(n-1, n)$ is a given function.

Example 4.7. Let us revisit Example4.5 by choosing $\alpha(t, \tau)=\frac{t^{2}}{2}$ and $x(t)=t^{4}$ with $t \in[0,1]$. Table 4.1 shows the approximated values obtained by our Chebfun function leftCaputo $(x, a l p h a, a, n)$ and the exact values computed with the formula given by Lemma 40. Table 4.1 was obtained using the following MATLAB code:

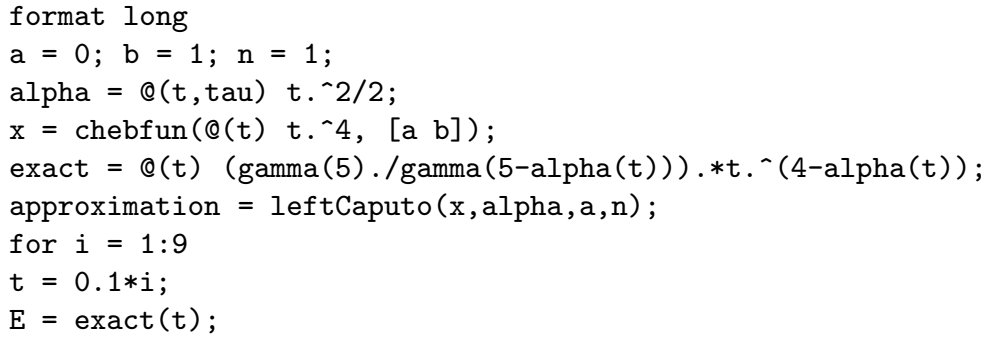




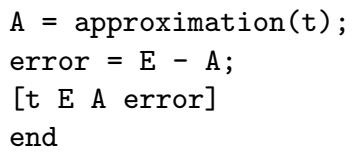

\begin{tabular}{|c|c|c|c|}
\hline $\mathbf{t}$ & Exact Value & Approximation & Error \\
\hline 0.1 & $1.019223177296953 \mathrm{e}-04$ & $1.019223177296974 \mathrm{e}-04$ & $-2.046431600566390 \mathrm{e}-18$ \\
\hline 0.2 & 0.001702793965464 & 0.001702793965464 & $-2.168404344971009 \mathrm{e}-18$ \\
\hline 0.3 & 0.009148530806348 & 0.009148530806348 & $3.469446951953614 \mathrm{e}-18$ \\
\hline 0.4 & 0.031052290994593 & 0.031052290994592 & $9.089951014118469 \mathrm{e}-16$ \\
\hline 0.5 & 0.082132144921157 & 0.082132144921157 & $6.522560269672795 \mathrm{e}-16$ \\
\hline 0.6 & 0.185651036003120 & 0.185651036003112 & $7.938094626069869 \mathrm{e}-15$ \\
\hline 0.7 & 0.376408251363662 & 0.376408251357416 & $6.246059225389899 \mathrm{e}-12$ \\
\hline 0.8 & 0.704111480975332 & 0.704111480816562 & $1.587694420379648 \mathrm{e}-10$ \\
\hline 0.9 & 1.236753486749357 & 1.236753486514274 & $2.350835082154390 \mathrm{e}-10$ \\
\hline
\end{tabular}

Table 4.1. Exact values obtained by Lemma 40 for functions of Example 4.7 versus computational approximations obtained using the Chebfun code.

Computational experiments similar to those of Example 4.7, obtained by substituting Lemma 40 by Lemma 41 and our leftCaputo routine by the rightCaputo one, reinforce the validity of our computational methods. In this case, we assume that the fractional order depends only on the second variable: $\alpha_{n}(\tau, t):=\bar{\alpha}_{n}(t)$, where $\bar{\alpha}_{n}:[a, b] \rightarrow(n-1, n)$ is a given function.

\section{A.3 Higher-order combined fractional Caputo derivative}

The higher-order combined Caputo fractional derivative combines both left and right Caputo fractional derivatives, that is, we make use of functions leftCaputo ( $x$, alpha, $a, n$ ) and rightCaputo ( $x$, alpha, $b, n$ ) provided in Section A.1 to define Chebfun computational code for the higher-order combined fractional Caputo derivative of variable-order:

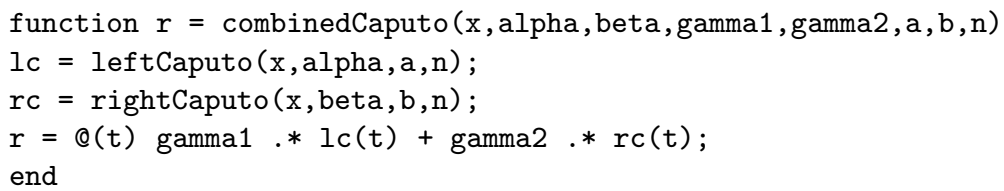

Then we illustrate the behavior of the combined Caputo fractional derivative of variable-order for different values of $t \in(0,1)$, using MATLAB.

Example 4.8. Let $\alpha(t, \tau)=\frac{t^{2}+\tau^{2}}{4}, \beta(t, \tau)=\frac{t+\tau}{3}$ and $x(t)=t, t \in[0,1]$. We have $a=0, b=1$ and $n=1$. For $\gamma=\left(\gamma_{1}, \gamma_{2}\right)=(0.8,0.2)$, we have ${ }^{C} D_{\gamma}^{\alpha(\cdot, \cdot), \beta(\cdot, \cdot)} x(0.4) \approx 0.7144$ : 


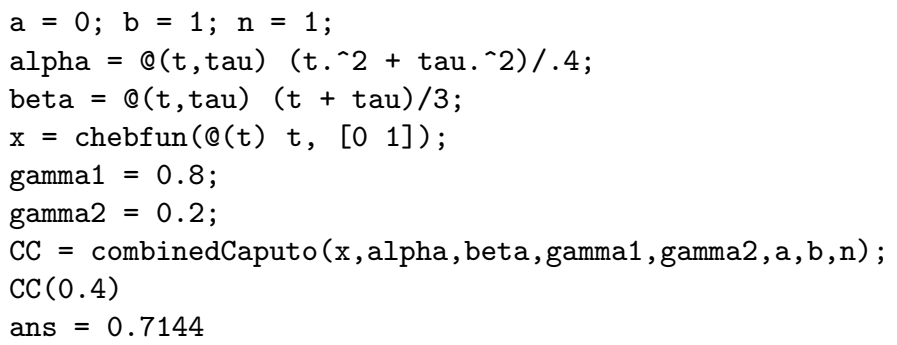

For other values of ${ }^{C} D_{\gamma^{n}}^{\alpha(\cdot, \cdot), \beta(\cdot, \cdot)} x(t)$, for different values of $t \in(0,1)$ and $\gamma=\left(\gamma_{1}, \gamma_{2}\right)$, see Figure 4.5 and Table 4.2 .

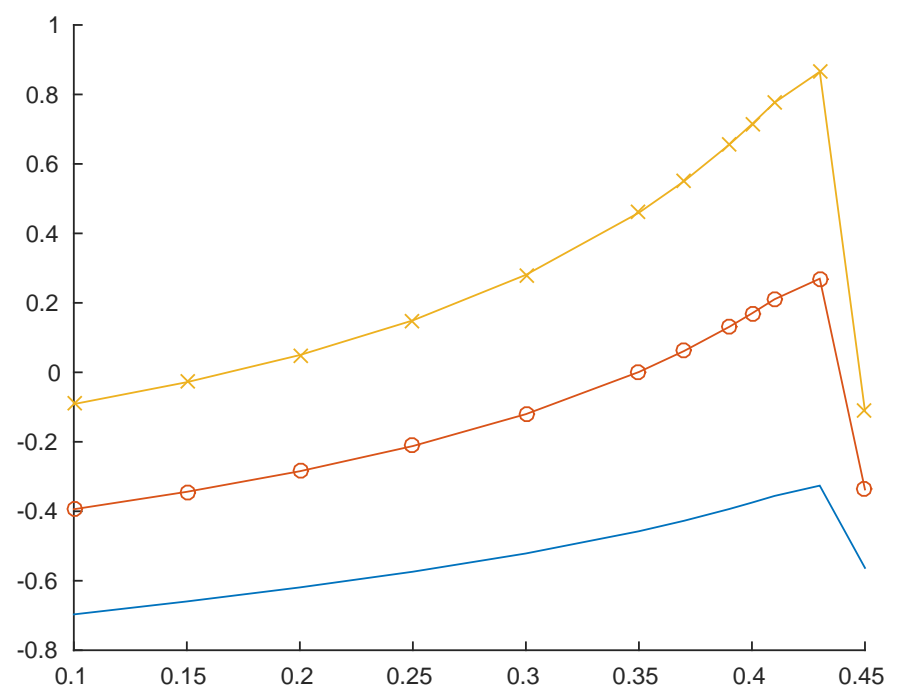

Fig. 4.5. Combined Caputo fractional derivative ${ }^{C} D_{\gamma}^{\alpha(\cdot, \cdot), \beta(\cdot, \cdot)} x(t)$ for $\alpha(\cdot, \cdot), \beta(\cdot, \cdot)$ and $x(t)$ of Example 4.8 continuous line for $\gamma=\left(\gamma_{1}, \gamma_{2}\right)=(0.2,0.8)$, "o-" style for $\gamma=\left(\gamma_{1}, \gamma_{2}\right)=(0.5,0.5)$, and " $\times-$ " style for $\gamma=\left(\gamma_{1}, \gamma_{2}\right)=(0.8,0.2)$.

\section{References}

Driscoll TA, Hale N, Trefethen LN (2014) Chebfun Guide. Pafnuty Publications, Oxford

Linge S, Langtangen HP (2016) Programming for ComputationsMATLAB/Octave. A Gentle Introduction to Numerical Simulations with MATLAB/Octave. Springer, Cham 


\begin{tabular}{|c|c|c|c|}
\hline $\mathbf{t}$ & Case 1 & Case 2 & Case 3 \\
\hline 0.4500 & -0.5630 & -0.3371 & -0.1112 \\
\hline 0.5000 & -790.4972 & $-1.9752 \mathrm{e}+03$ & $-3.1599 \mathrm{e}+03$ \\
\hline 0.5500 & $-3.5738 \mathrm{e}+06$ & $-8.9345 \mathrm{e}+06$ & $-1.4295 \mathrm{e}+07$ \\
\hline 0.6000 & $-2.0081 \mathrm{e}+10$ & $-5.0201 \mathrm{e}+10$ & $-8.0322 \mathrm{e}+10$ \\
\hline 0.6500 & $2.8464 \mathrm{e}+14$ & $7.1160 \mathrm{e}+14$ & $1.1386 \mathrm{e}+15$ \\
\hline 0.7000 & $4.8494 \mathrm{e}+19$ & $1.2124 \mathrm{e}+20$ & $1.9398 \mathrm{e}+20$ \\
\hline 0.7500 & $3.8006 \mathrm{e}+24$ & $9.5015 \mathrm{e}+24$ & $1.5202 \mathrm{e}+25$ \\
\hline 0.8000 & $-1.3648 \mathrm{e}+30$ & $-3.4119 \mathrm{e}+30$ & $-5.4591 \mathrm{e}+30$ \\
\hline 0.8500 & $-1.6912 \mathrm{e}+36$ & $-4.2280 \mathrm{e}+36$ & $-6.7648 \mathrm{e}+36$ \\
\hline 0.9000 & $5.5578 \mathrm{e}+41$ & $1.3895 \mathrm{e}+42$ & $2.2231 \mathrm{e}+42$ \\
\hline 0.9500 & $1.5258 \mathrm{e}+49$ & $3.8145 \mathrm{e}+49$ & $6.1033 \mathrm{e}+49$ \\
\hline 0.9900 & $1.8158 \mathrm{e}+54$ & $4.5394 \mathrm{e}+54$ & $7.2631 \mathrm{e}+54$ \\
\hline
\end{tabular}

Table 4.2. Combined Caputo fractional derivative ${ }^{C} D_{\gamma}^{\alpha(\cdot, \cdot), \beta(\cdot, \cdot)} x(t)$ for $\alpha(\cdot, \cdot), \beta(\cdot, \cdot)$ and $x(t)$ of Example 4.8. Case 1: $\gamma=\left(\gamma_{1}, \gamma_{2}\right)=(0.2,0.8)$; Case 2: $\gamma=\left(\gamma_{1}, \gamma_{2}\right)=$ $(0.5,0.5)$; Case 3: $\gamma=\left(\gamma_{1}, \gamma_{2}\right)=(0.8,0.2)$.

Trefethen LN (2013) Approximation Theory and Approximation Practice. Society for Industrial and Applied Mathematics 


\section{Index}

admissible trajectory, 22

admissible variation, 23

approximation error, 45, 49, 52, 53

beta function, 4, 41, 65, 113

boundary conditions, 22, 24, 55, 58, 68,

$69,75,83,87,92,96,101$

brachistochrone, 22

Caputo fractional derivative

left, 7

left (higher-order), 63, 64

left (variable-order), 14

partial left, 44

partial right, 44

right, 7

right (higher-order), 63

right (variable-order), 14

several variables, 42

combined Caputo fractional derivative

classical, 12

higher-order, 66

variable-order, 62

combined Riemann-Liouville fractional derivative

higher-order, 65

variable-order, 62

dual parameter set, 17

Euler-Lagrange equations, 23, 28, 30, $69,76,83,87,92,96,102$

expansion formulas, $45,49,51-53$

extremal, 24 first variation, 23

gamma function, 4, 113

generalized fractional derivative of Caputo, 15

of Riemann-Liouville, 15

generalized fractional integrals

of Riemann-Liouville, 14

Grünwald-Letnikov fractional derivative, 9

Hadamard fractional derivative left, 9

right, 9

Hadamard fractional integral left, 6

right, 6

holonomic constraint, 95, 96

horizontal terminal line, 73

integration by parts

Caputo fractional derivative of higher-order, 66

Caputo fractional derivatives, 16, 68

Caputo fractional derivatives of variable-order, 16

fractional integrals of variable-order, 16

Riemann-Liouville fractional integrals, 16

isoperimetric problem, 25, 86

Lagrange multiplier, 26 
Mittag-Leffler function one parameter, 5

two parameters, 5

psi function, 4

Riemann-Liouville fractional derivative left, 7

left (higher-order), 63

left (variable-order), 13

right, 7

right (higher-order), 63

right (variable-order), 13

Riemann-Liouville fractional integral left, 6

left (higher-order), 63

left (variable-order), 13

right, 6

right (higher-order), 63

right (variable-order), 13
Riesz fractional derivative, 11

Riesz fractional integral, 11

Riesz-Caputo fractional derivative, 11

symmetric fractional derivative, 11

tautochrone problem, 2, 27

terminal cost function, $69,75,87,96$

terminal curve, 73

time delay, 82

transversality conditions, 24, 25, 29, 69, $72,76,81,88,91,93,97,102,105$

variational fractional problem, 28, 29

fundamental, 69

higher-order, 75

of Herglotz, 101

with a holonomic constraint, 95

with time delay, 83

vertical terminal line, 73 